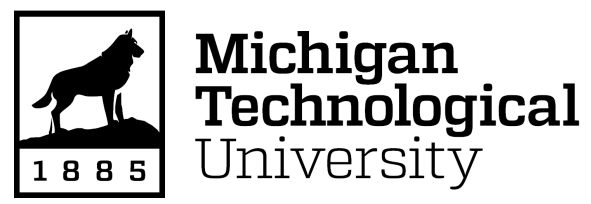

Michigan Technological University Digital Commons @ Michigan Tech

Dissertations, Master's Theses and Master's Reports

2020

\title{
ARTIFICIAL SYNTHETIC SCAFFOLDS FOR TISSUE ENGINEERING APPLICATION EMPHASIZING THE ROLE OF BIOPHYSICAL CUES
}

Samerender Nagam Hanumantharao

Michigan Technological University, snagamha@mtu.edu

Copyright 2020 Samerender Nagam Hanumantharao

\section{Recommended Citation}

Nagam Hanumantharao, Samerender, "ARTIFICIAL SYNTHETIC SCAFFOLDS FOR TISSUE ENGINEERING APPLICATION EMPHASIZING THE ROLE OF BIOPHYSICAL CUES", Open Access Dissertation, Michigan Technological University, 2020.

https://doi.org/10.37099/mtu.dc.etdr/1088

Follow this and additional works at: https://digitalcommons.mtu.edu/etdr

Part of the Biology and Biomimetic Materials Commons, Biomaterials Commons, Molecular, Cellular, and Tissue Engineering Commons, Nanoscience and Nanotechnology Commons, and the Polymer and Organic Materials Commons 
ARTIFICIAL SYNTHETIC SCAFFOLDS FOR TISSUE ENGINEERING APPLICATION EMPHASIZING THE ROLE OF BIOPHYSICAL CUES

By

Samerender Nagam Hanumantharao

\begin{abstract}
A DISSERTATION
Submitted in partial fulfillment of the requirements for the degree of DOCTOR OF PHILOSOPHY

In Biomedical Engineering
\end{abstract}

MICHIGAN TECHNOLOGICAL UNIVERSITY

2020

(C) 2020 Samerender Nagam Hanumantharao 
This dissertation has been approved in partial fulfillment of the requirements for the Degree of DOCTOR OF PHILOSOPHY in Biomedical Engineering.

\title{
Department of Biomedical Engineering
}

\author{
Dissertation Advisor: $\quad$ Dr. Smitha Rao \\ Committee Member: $\quad$ Dr. Megan Frost \\ Committee Member: Dr. Orhan Soykan \\ Committee Member: $\quad$ Dr. Jeremy Goldman
}

Department Chair: Dr. Sean Kirkpatrick 


\section{Table of Contents}

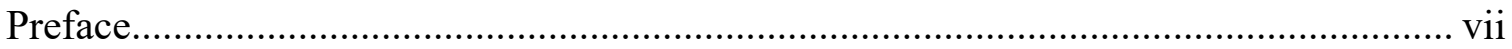

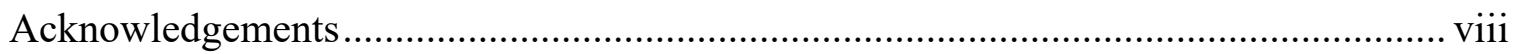

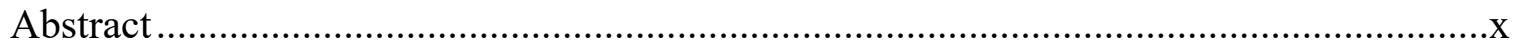

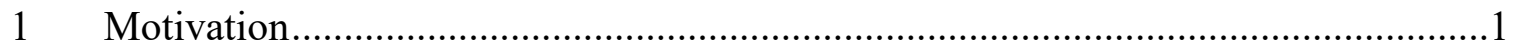

2 Multi-Functional Electrospun Nanofibers from Polymer Blends for Scaffold Tissue

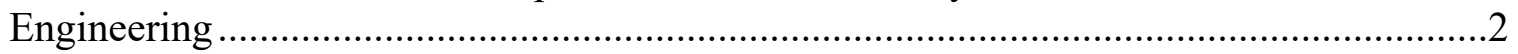

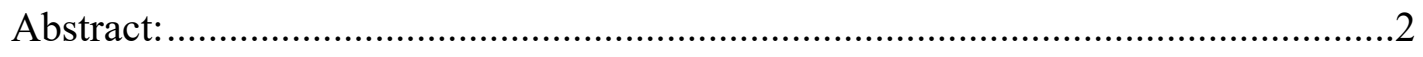

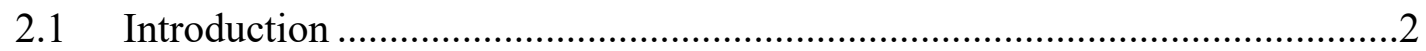

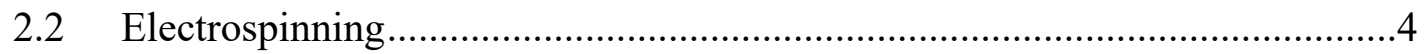

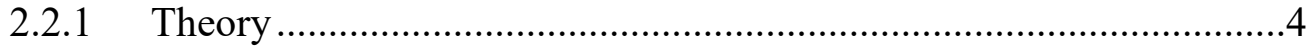

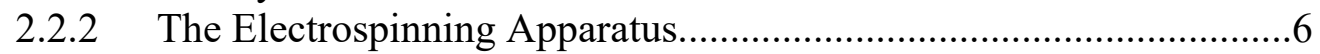

2.2.2.1 Changes in Collector Design ............................................

2.2.2.1.1 Rotating Mandrel .........................................6

2.2.2.1.2 Patterned Collector.........................................

2.2.2.1.3 Gap Electrospinning.....................................

2.2.2.1.4 Magnetic Field Associated

Electrospinning ……..................................................

2.2.2.1.5 Wet Spinning .............................................

2.2.2.2 Changes in Orientation ………………….......................

2.2.2.2.1 Vertical Electrospinning ...............................9

2.2.2.2.2 Horizontal Electrospinning ……………......10

2.2.2.2.3 Converse Electrospinning ……………….....10

2.2.2.3 Changes in Spinneret .....................................................10

2.2.2.3.1 Coaxial Electrospinning ..............................10

2.2.2.3.2 Co-Electrospinning ......................................13

2.2.2.3.3 In-Line Polymer Blending ...........................14

2.2.2.4 Other Modifications ...........................................................

2.2.2.4.1 Centrifugal Electrospinning .......................14

2.2.2.4.2 Near Field Electrospinning …......................14

2.2.2.4.3 Needleless Electrospinning ..........................15

2.2.2.4.4 Emulsion Electrospinning...........................16

2.3 Polymer Blends for Tissue Scaffold Engineering .........................................17

2.3.1 Natural Polymer Blends ...............................................................19

2.3.2 Synthetic Polymer Blends...............................................................22

2.3.3 Mixed Polymer Blends ................................................................26

$2.4 \quad$ Perspectives and Conclusions .................................................................. 
3 Self-Assembly of 3D Nanostructures in Electrospun Polycaprolactone-Polyaniline Fibers and their Application as Scaffolds for Tissue Engineering ....................................46

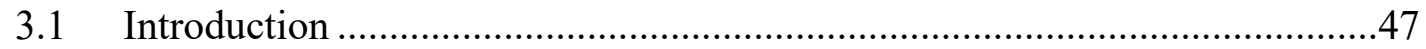

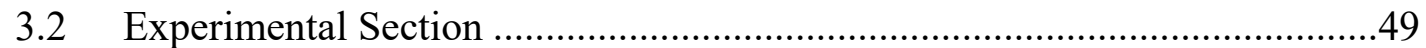

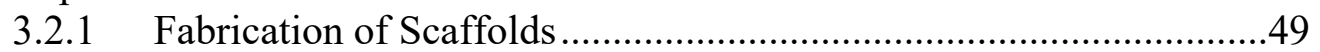

3.2.2 Characterization of scaffolds ..........................................................49

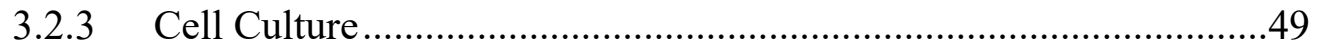

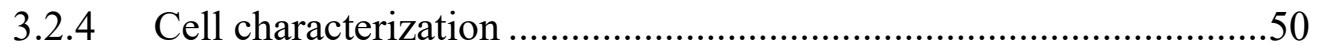

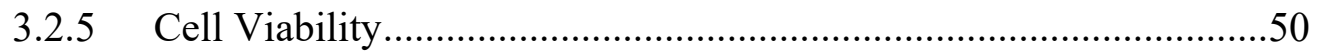

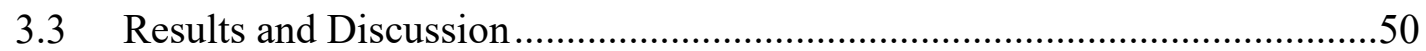

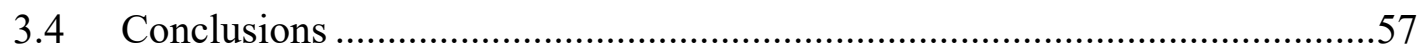

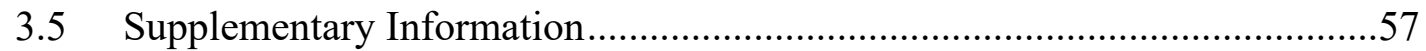

4 Engineered three-dimensional scaffolds modulating fate of breast cancer cells using stiffness and morphology related cell adhesion ..................................................................64

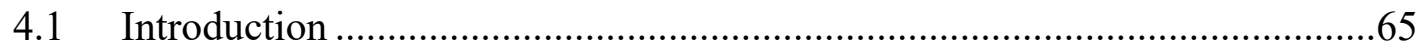

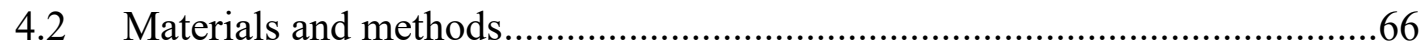

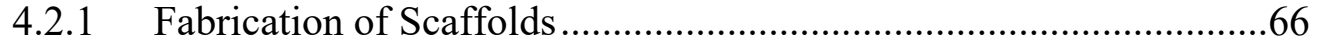

4.2.2 Characterization of Scaffolds........................................................66

4.2.3 Cell Culture, Seeding, Viability, and Immunochemistry...................67

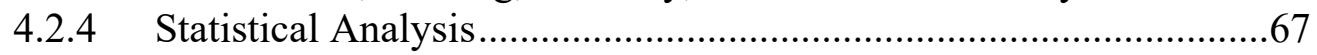

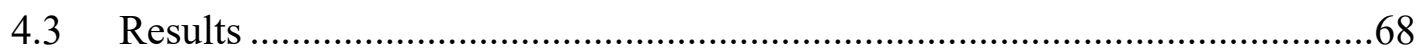

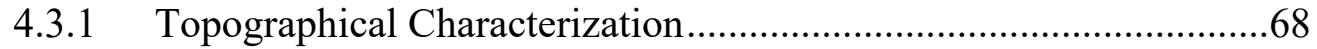

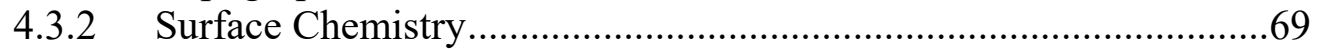

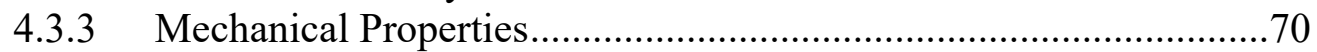

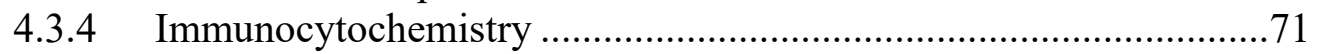

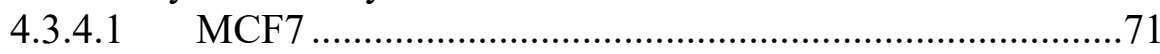

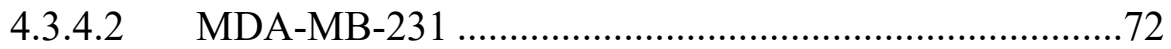

4.3.4.3 MCF10AneoT ..........................................................73

4.3.5 Cell Viability..............................................................................

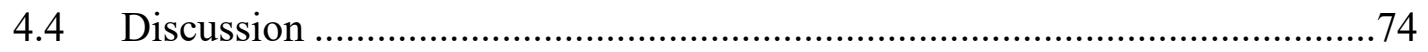

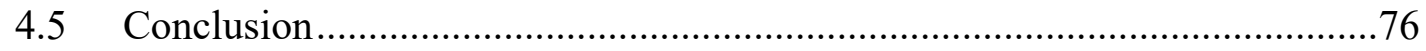

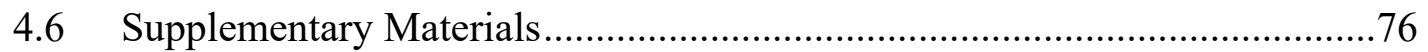

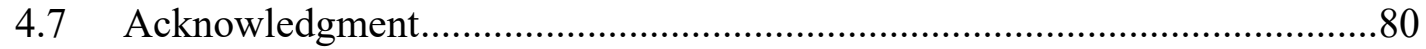

5 Scaffold morphology affects proliferation of renal, lung and bladder cancer cells...81

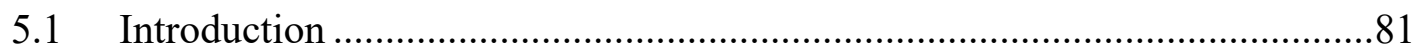

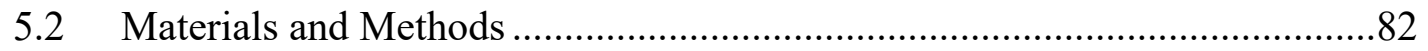

5.2.1 Fabrication and characterization of scaffolds ..................................82 


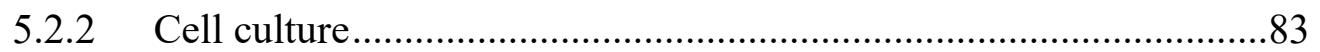

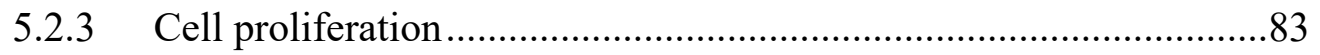

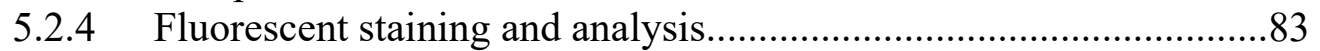

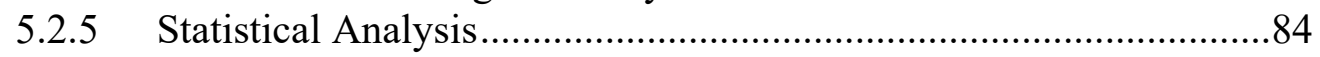

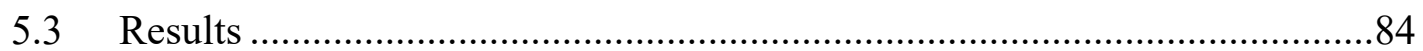

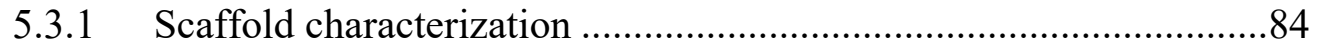

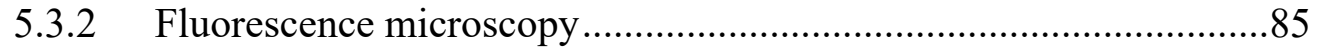

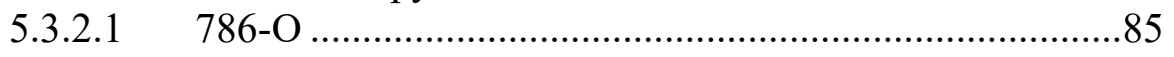

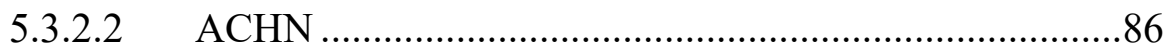

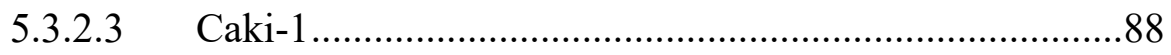

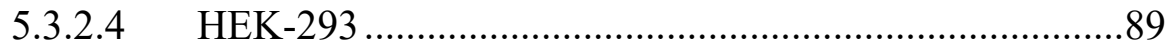

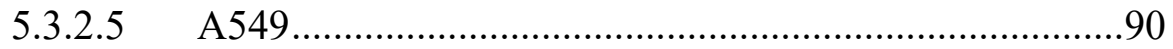

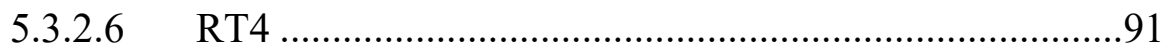

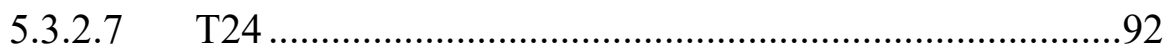

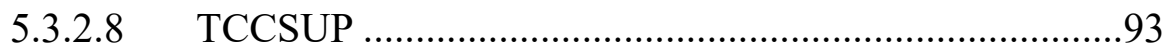

5.3.3 Cell Proliferation...........................................................................94

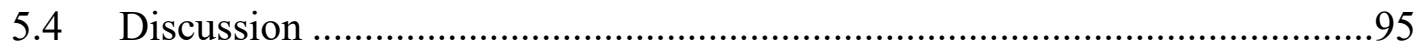

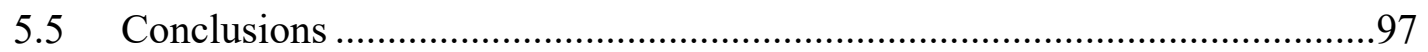

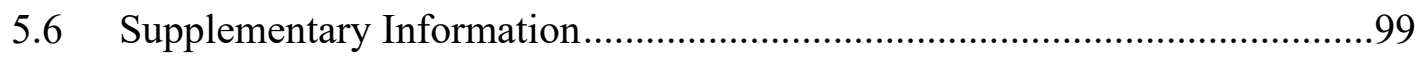

6 A novel PCL scaffold based metabolic regulator in cancer cells ...........................106

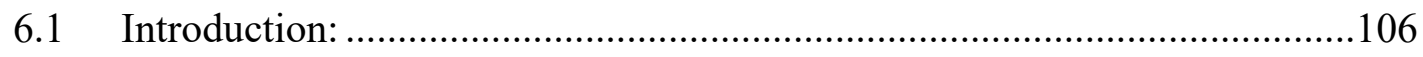

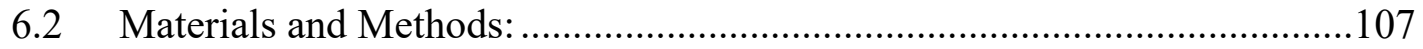

6.2.1 Synthesis of GLUT5-specific probe ………….............................107

6.2.2 Fabrication and characterization of scaffolds ................................108

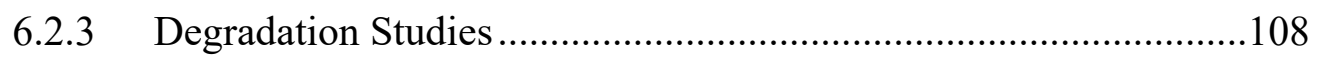

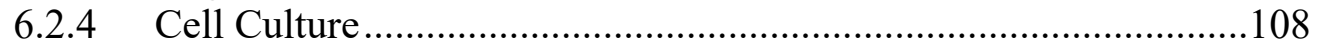

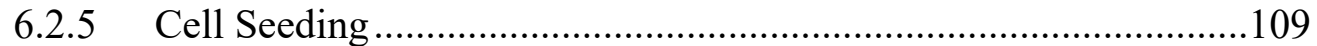

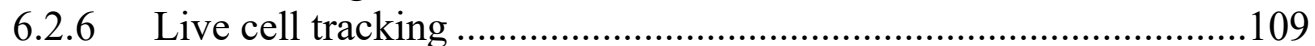

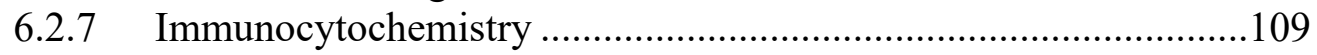

6.2.8 Fluorescent Staining................................................................. 110

6.2.9 Cell metabolism: .......................................................................

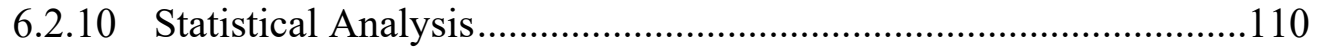

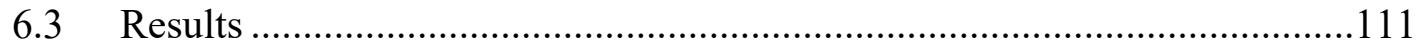

6.3.1 Scaffold fabrication and characterization ......................................111

6.3.2 Degradation study .....................................................................113

6.3.3 Live cell tracking ………….....................................................113

6.3.4 Protein expression....................................................................114

6.3.4.1 Cytokeratin 18........................................................114

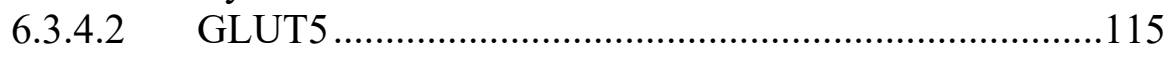

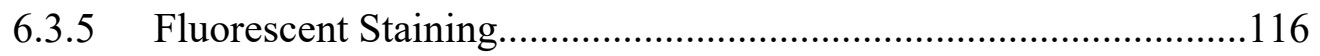

6.3.6 Cell Metabolism........................................................................117 


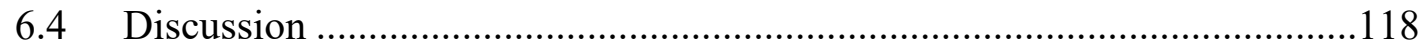

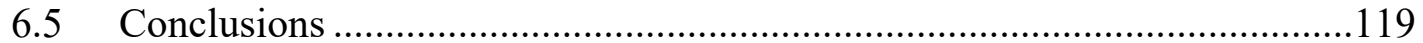

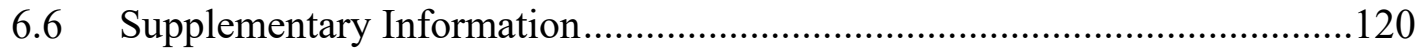

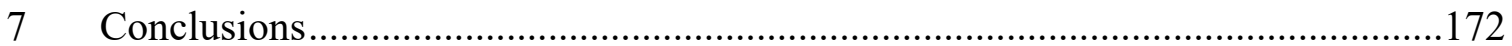

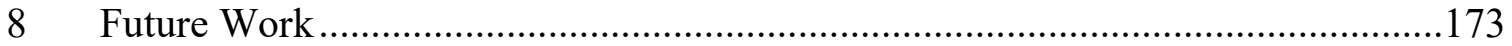

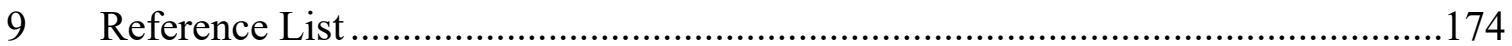

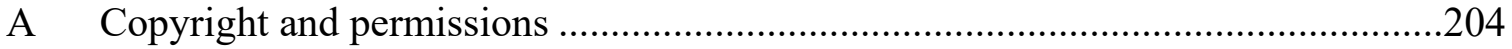




\section{Preface}

Chapter 2 is part of a review paper titled 'Multi-functional electrospun nanofibers from polymer blends for scaffold tissue engineering' published in MDPI fibers journal which is an open access journal co-authored with Dr. Smitha Rao whose responsibilities were project administration, writing-review and editing, supervision.

Chapter 3 is part of an original research paper titled 'Self-assembly of 3D nanostructures in electrospun polycaprolactone-polyaniline fibers and their application as scaffolds for tissue engineering' co-authored with undergraduate student in the lab Carolynn Que. Carolynn Que helped with cell seeding experiments and fluorescent staining and imaging.

Chapter 4 is part of an original research article published in IEEE OJEMBS titled 'Engineered Three-Dimensional Scaffolds Modulating Fate of Breast Cancer Cells Using Stiffness and Morphology Related cell Adhesion' along with co-authors then undergraduate student Brennan Vogl and graduate student Carolynn Que. Brennan Vogl helped in imaging of fluorescently stained cells, cell culture experiments, and postprocessing images. Carolynn Que helped in cell culture experiments, electron microscopy, imaging of fluorescently stained cells and post-processing images.

Chapter 5 was completed in part with the help of Carolynn Que who helped in imaging of the fluorescently stained cells, scaffold fabrication and cell culture experiments.

Chapter 6 was completed with the help of Stephanie Bule who aided in live-cell imaging, immunofluorescent staining and imaging, and cell culture imaging, Carolynn Que who helped with the cell culture experiments, scaffold fabrication, metabolic studies, scaffold processing and Brennan Vogl who helped in cell culture experiments and imaging. The ManCou-H probes used in this chapter was prepared by Dr. Marina Tanasova's lab group. 


\section{Acknowledgements}

The journey leading to the culmination of my dissertation was enriched by several faculty members, colleagues, friends, and family. I am indebted to each one of them.

First and foremost, I would like to thank my advisor, Dr. Smitha Rao, for her patient, proactive and insightful mentoring and for being very approachable to my questions and concerns at all times. I will always cherish our conversations about science and about the nuances of navigating the academic world.

I am grateful to my Ph.D. committee members, Dr. Megan Frost, Dr. Orhan Soykan and Dr. Jeremy Goldman for their support and advice. A special thanks to Dr. Goldman for training me on animal studies. Dr. Frost has embodied the roles of a mentor, a guide for laboratory work and a teacher. For this, I am very grateful to her.

Mr. Owen Mills has played a pivotal role in training me on Field Emission Scanning Electron Microscopy and has extended a helping hand in the characterization of polymeric scaffolds. Dr. Kathryn A. Perrine has played an integral part in teaching me the applications of ATR-FTIR and its uses. Mr. Paul Fraley and Dr. Chito Kendrick's training on Dynamic Mechanical Analysis and Microfabrication respectively were invaluable. Dr. Feng Zhao has been an irreplaceable mentor and teacher, whose assistance in lab was very pertinent and useful. I owe my gratitude to all of them.

My peers and colleagues are a constant source of motivation, inspiration, and good cheer. Their presence and support made the trying times in lab more bearable. Srinivas Kannan has been a source of unwavering support; whose honest and timely feedback were of immense help since high school. Ms. Stephanie Bule, a reliable colleague, partnered with me on several projects and co-curricular activities. Ms. Kristine Fink is a friend and my first mentee. I thank her for being patient with me. This dissertation would not have come into fruition without the significant contribution of Ms. Carolynn Que. I am very thankful to her for her indispensable assistance and patience. I immensely appreciate the contributions and support of the Biomedical Microdevices lab group and my co-authors. Special thanks to Maria for lending a helping hand to some of my experiments and for guiding me through the Experimental Biomedical Techniques lab course.

I am grateful to the Coordinators and colleagues at the Department of Biomedical Engineering, for their administrative assistance. I would also like to thank Ms. Stacey Sedar, Ms. Coreen Dompier, Ms. Devin Seppala, for their timely help throughout the duration of my $\mathrm{PhD}$ program.

I owe my gratitude to all the faculty and professors at Michigan Technological University for being very approachable and for providing myriad clarifications related to academics and administrative operations. I am thankful to Dr. Padmalathika Varanasi for helping me proofread documents and lending a patient ear to my complaints. 
My journey was made all the more fulfilling thanks to the friendship and support of several people around the world, who have been unwavering cheerleaders and harbingers of good times. I am immensely grateful to Nancy Byers Sprague and Dianne Sprague for their kindness, immeasurable generosity, warmth and for introducing me to sailing. I am thankful to friends and volunteers at the Little Brothers-Friends of the Elderly, especially Aloïs, Marion, and Selina, and to Dr. John Adler for their goodwill and delightful companionship. I am grateful for the company and support provided by my friends at Michigan Technological University with a special mention to Salil, Anindya, Omkar, Ajit, Marjan, Nupur, Priyanka and Rachel for their support at various stages of my graduate school education.

My mentors, teachers, friends, and family in India have been my constant pillars of strength and support. My friends from school have been my trustworthy and loyal companions, who are ready to help me at any point, without any expectations. A special thanks to my friends from school, Vivek Narayanan, Sudarshan, Prashanth, Ramyya, Rajalakshmi, Siddarth Krishnan and Sruthi. My friends from my undergraduate program, Venkatraman, Nideesh, Mithreyi, Shrijha, Shruti Suresh, and Ramya A encouraged me to be resolute and follow my ambitions. My life would have a big void without them. Team research has yielded some of the greatest breakthroughs known in science. I would like to acknowledge the contribution of my first research team consisting of Bhaavana Krishnan and Siva Kumar, who showed me the endless possibilities of tackling a research problem as a team and my senior design team members Adharshini and Meghana.

My childhood friends, Elangkumaran and Divyabharathi, have been my steadfast friends, confidants and continue to be an indelible part of my life. They have always had my back and are the very definition of friends like family.

My professor from my undergraduate program, Dr. Thiagarajan Raman, enhanced my knowledge in Biomedical Engineering and motivated me to advance in my academic pursuits. I am very grateful to Dr. Shareef Kolathodi, Dr. Milan Palei and Dr. T.S. Natarajan for providing me with an opportunity to work on supercapacitors. This project was one of my early forays into research and was instrumental in developing an insatiable appetite to seek the unknown and find solutions.

I am very lucky to be unconditionally loved, nurtured, and guided by my family, whose positivity and boundless strength has been the reason for me to persevere and overcome challenges. My mother, Ms. Hamsa Thara, my father, Mr. Hanumantha Rao, my grandfather, Mr. Varadarajulu and my uncle, Mr. Hari Krishnan, are my anchors and I dedicate my dissertation to them.

I graduate into the future with my curiosity piqued, eager to make further scientific inroads in my career, armed with the goodwill, confidence, and zest for life that I have gained in my Ph.D. journey. 


\section{Abstract}

The mechanotransduction of cells is the intrinsic ability of cells to convert the mechanical signals provided by the surrounding matrix and other cells into biochemical signals that affect several distinct processes such as tumorigenesis, wound healing, and organ formation. The use of biomaterials as an artificial scaffold for cell attachment, differentiation and proliferation provides a tool to modulate and understand the mechanotransduction pathways, develop better in vitro models and clinical remedies. The effect of topographical cues and stiffness was investigated in fibroblasts using polycaprolactone (PCL)- Polyaniline (PANI) based scaffolds that were fabricated using a self-assembly method and electrospinning. Through this method, scaffolds of different topography and stiffness were fabricated with similar surface chemistries. The effect of scaffold morphologies on the cells were investigated. PCL scaffolds of three distinct morphologies- honeycomb, aligned and mesh were used with similar surface chemistry to investigate the changes in cell behavior of breast, renal, lung and bladder cancer to the physical cues. Selective adhesion and localization of cells to specific morphologies were determined. In order to demonstrate the scaffold as a source of biochemical signals, ManCou-H, capable of targeting the fructose-specific glucose transporter GLUT5 was electrospun with the scaffolds of different morphologies. The PCL scaffolds were used as the backbone to release ManCou-H and changes in protein expression and metabolic activity was characterized. The findings made available through this research will help in the design of better cell-specific in vitro model systems to better understand cellular responses to clinical therapies, assess cell response to specific mechanical and chemical cues. 


\section{Motivation}

The cellular microenvironment provides cues for the cells in the form of biophysical cues (matrix stiffness, alignment, porosity, elasticity) and biochemical cues (matrix composition). These signals together dictate complex cellular functions such as cell differentiation, cell migration and apoptosis. Additionally, dynamic biophysical cues such as electric stimulation or biochemical cues such as hormones or growth factors help in providing functionality to tissues and help in harmonizing homeostasis. Hence, these cues have been widely investigated for tissue regeneration and cancer tissue engineering. Recent technological advances have enabled researchers to comprehensively investigate the role of biophysical cues by designing artificial matrices to provide better control of the parameter being tested.

In my dissertation, I have focused on the use of synthetic polymers with controlled topographies investigating the role of biophysical cues on cells. The scaffolds or artificial matrices with structural differences are fabricated using electrospinning, without any postprocessing from the starting polymer solution by varying the electrospinning conditions alone. Hence, these scaffolds provide the cells with the same surface chemical moieties but varying physical cues, thereby creating a controlled environment to investigate the effect of biophysical cues alone on cells. The scaffolds fabricated have been used in understanding the biophysical effects on fibroblasts, epithelial cells, and carcinomas at different stages in vitro. This study will help in designing efficient scaffolds for wound healing and understanding the cell-signaling cascades influenced by the biophysical cues, leading to cell-specific platforms for use in investigating microenvironments and possible discovery of therapeutic targets. 


\title{
2 Multi-Functional Electrospun Nanofibers from Polymer Blends for Scaffold Tissue Engineering
}

\author{
Samerender Nagam Hanumantharao and Smitha Rao * \\ Department of Biomedical Engineering, Michigan Technological University, Houghton, \\ MI 49931, USA \\ "Corresponding author:Email: smithar@mtu.edu
}

Fibers 2019, 7(7), 66; https://doi.org/10.3390/fib7070066

Received: 27 May 2019 / Revised: 24 June 2019 / Accepted: 12 July 2019 / Published: 19 July 2019

\begin{abstract}
Electrospinning and polymer blending have been the focus of research and the industry for their versatility, scalability, and potential applications across many different fields. In tissue engineering, nanofiber scaffolds composed of natural fibers, synthetic fibers, or a mixture of both have been reported. This review reports recent advances in polymer blended scaffolds for tissue engineering and the fabrication of functional scaffolds by electrospinning. A brief theory of electrospinning and the general setup as well as modifications used are presented. Polymer blends, including blends with natural polymers, synthetic polymers, mixture of natural and synthetic polymers, and nanofiller systems, are discussed in detail and reviewed.
\end{abstract}

Keywords: electrospinning; nanofibers; tissue scaffold engineering; polymer blending; functional nanofibers

\subsection{Introduction}

The field of tissue engineering involves the fabrication of artificial tissues, artificial organs, and tissue regeneration [1]. Scaffolds are used as templates for tissue engineering studies and are composed of decellularized tissue matrices and synthetic or natural polymer constructs [2]. The scaffolds provide mechanical support as well as the physical cues within the microenvironment, aiding cell growth. To enable optimum conditions, it is necessary to mimic the natural microenvironment. This includes the mechanical properties, such as topography, architecture, elasticity, and stiffness, and biochemical factors, such as surface chemistry, growth factors, and cytokines. This need for mimicking the extracellular matrix $(\mathrm{ECM})$ has driven the design of novel biomaterials and fabrication techniques. The readers are encouraged to refer to some of the recent reviews on this topic [3-9].

While several innovative approaches for tissue engineering have been developed within the last decade, electrospinning has continued to be the most commonly used. The advantages of electrospinning are its versatility, flexibility, ability to use different polymer 
combinations, and ability to upscale easily. Electrospinning is the modification of the electrospraying process where electrostatic fields are used for liquid atomization to form droplets (Figure 1 and 2A). Since mechanical forces are not used for the formation of fibers, it is also known as hydrodynamic jetting. While the effect of electric fields on droplets have been known since the 17th century and was patented in 1900 by J.F. Cooley [10], practical applications were not well developed. In the latter part of the 20th century Doshi and Reneker demonstrated the versatility of the technique by fabricating fibers from organic polymer solutions [11,12]. The typical setup is straightforward and provides flexibility over the fabrication method. Scaling the system for mass manufacturing is relatively easy and all the advantages of the approach translate effectively. The common features of the set-up include a voltage source, syringe pumps, and a collector. For all forms of electrospinning, an initial sol-gel composed of polymers in suitable solvents is needed. The use of one or more polymers with compatible solvents has been reported [13-15].

Polyblending or polymer blending is an effective strategy to blend polymers by incorporating the unique properties of the component polymers or to create novel materials [16]. They can be sub-classified based on their miscibility as homogenous (miscible blend) and heterogenous (immiscible) polymer blends. Miscible blends exhibit a single glass transition temperature while immiscible polymer blends exhibit glass transition temperatures of the individual polymer components. Miscible blends usually form clear solutions, while immiscible blends form cloudy solutions. Furthermore, immiscible blends can have a separation of phases if there is no mechanical stimulus. Polymer blends can be distinguished from block and graft polymers because of the formation of chemical bonds to prepare new polymer systems in the latter. The blending of polymers is typically achieved using approaches such as extrusion, mixing, and injection molding, to make sure the polymers are blended correctly and do not separate out. The thermodynamics of the polymer systems play a major role in determining the miscibility of polymer blends. Some of the parameters that can be modified in order to transition from the preparation of a miscible polymer blend to an immiscible polymer blend are molecular weights of the individual polymer components, solubility of the solvents used for making polymer solutions, temperature, and the ratio of the polymer components in the polymer blend. The major challenge involved in the blending of polymers is that most polymers are thermodynamically immiscible and hence separate out unless stabilized using fillers. It is essential that the blends remain stable in order to create functional materials with the desired properties. The advantage of electrospinning is that functional nanofibers can be fabricated from both miscible and immiscible blends. In this review, we will focus on the fabrication of functional scaffolds through the preparation of novel polymer blends and electrospinning. The modifications made to the electrospinning systems are highlighted. The different polymer blends and the method of fabrication are discussed in detail in the later sections. 


\subsection{Electrospinning}

\subsubsection{Theory}

The generalized setup (Figure 1) consists of a voltage source, syringe pump, nozzle, grounded collector, and syringe. A high electric field is applied $(>0.5 \mathrm{kV} / \mathrm{cm})$ to the tip of the nozzle through which a polymer solution of compatible viscosity $(200-4000 \mathrm{cP})$ flows through. A grounded collector is used to collect the fibers. The process of electrospinning revolves around the deformation of the polymer droplet at the tip of the nozzle under the influence of an electric field forming a Taylor cone as seen in Figure 2A [17].

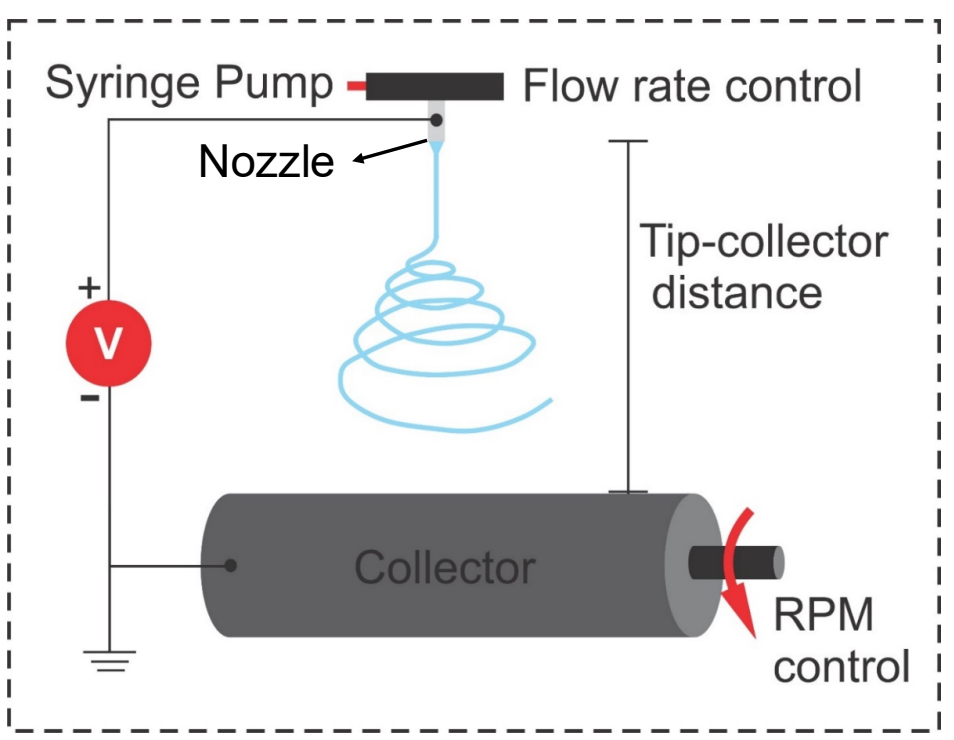

Figure 1. A basic illustration of the electrospinning set-up showing all the possible parameters that can be controlled. Adapted with permission from [18], Samerender Nagam Hanumantharao, 2017.

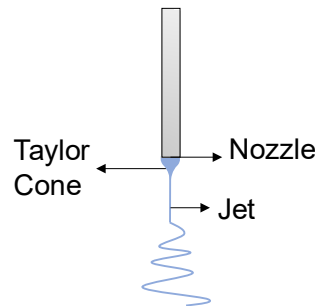

(A) Electrospinning

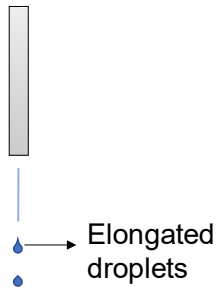

(B) Electrospraying

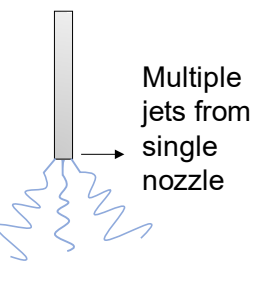

(C) Jet Splitting
Branching

$\rightarrow$ of jet into sub-jets

Figure 2. Schematic illustration of the behavior of jets during the electrospinning process.

The entire electrospinning process consists of three stages: Jet initiation, jet elongation, and the formation of nanofibers. The jet initiation starts after the formation of a Taylor cone. The formation of the Taylor cone occurs when the electric field can overcome the 
surface tension of the polymer solution, thus elongating it. There is a threshold limit to the voltage during which the shape of the Taylor cone changes gradually. A higher voltage causes the droplet to recede into the nozzle while a lower voltage does not create a Taylor cone [19]. The jet travels to the grounded collector or negatively charged collector after ejecting out from the Taylor cone in a linear path initially and later collects on top of it after chaotically moving from the nozzle towards the collector, caused by bending instabilities. The angle of the Taylor cone formed varies depending on the parameters used for electrospinning [20]. The jet initiation process begins when the electric field applied is above the critical voltage value. The critical voltage value is based on the surface tension of the polymer solution and its flow rate. The charge density is highest at the tip of the Taylor cone. Once the jet is initiated, it travels in a linear trajectory. Several parameters play a role in the jet elongation step. Environmental conditions, such as humidity and temperature, electrostatic forces, which are dependent on the electric field applied and the permittivity of the fluid, solvent evaporation rate, and viscoelastic response of the fluid are some of the parameters that affect the process [21].

The acceleration of the jet towards the collector is proportional to the electric field and flow rate. A steady jet is required for continuous electrospinning. The rate of decay of the jet depends on the molecular weight of the polymer and the evaporation rate of the solvent apart from the environmental conditions when the flow rate is constant. A low molecular weight polymer solution or a volatile solvent lead to a faster decay of the jet, leading to splattering or electro spraying (Figure 2B). The forces on the jet vary over time as the jet travels from the tip of the Taylor cone to the collector. Several forces act on the jet in different directions, leading to various fluid instabilities. Splitting of the jet into multiple jets occurs when there is a local charge accumulation on the tip of the jet, leading to branching into sub-jets in a process known as splaying (Figure 2D). Splaying is dependent on the physical and electrical properties of the solvent used and the molecular weight of the polymers. It also generally occurs when a relatively high electric field is used for electrospinning. The process of splaying in different solvents was reviewed in detail by Eda and colleagues [1]. However, in some cases when the electric field applied is higher than the critical value, it leads to receding of the Taylor cone into the nozzle and multiple jets emanating from a single nozzle. This process is known as jet splitting (Figure 2C). After following a linear trajectory, the jet undergoes chaotic flow, which is caused by a non-axisymmetric force or whipping instability [2]. These forces cause the jet to stretch and follow a helical path whose diameter keeps increasing. This provides enough time for the local charges on the tip of the jet to redistribute themselves because of Coulombic repulsion and allows the jet to become elongated and thin. The other instabilities acting on the jet during electrospinning and influencing the formation of nanofibers are the Rayleigh-Plateau instability and an axis-symmetric force. Rayleigh force acts on the jet on opposite sides perpendicular to the surface area of the jet while surface tension works to reduce the total surface area of the jet. This leads to the formation of elongated droplets as the charge on the droplets prevent them from coalescing (Figure 2B). The presence of droplets during electrospinning is not desired in many applications. Hence, a balance between Rayleigh instability and surface tension is needed for continuous electrospinning. The physical properties of the solvent used, and the viscosity of the polymer solution are 
key in maintaining the balance [3]. As the jet reaches the collector, it solidifies and forms nanofibers. The electric field applied, solvent used, and polymer concentration play a role in the solidification of the jet and evaporation of the solvents. A process called conglutination, where partially solidified jets lead to the production of fibers that are attached at points of contact, affects the morphology and mechanical properties of the fibers [4]. In the entire process, environmental conditions impact the formation of the Taylor cone, affecting the structure of the nanofibers (diameter, morphology, and mechanical properties). For example, higher humidity leads to the formation of pores on the surface of the nanofibers $[5,6]$. The entire process of electrospinning can be modified according to the requirements and reengineered to provide more control over the parameters in order to fabricate novel nanostructures. The theoretical models and mechanisms of electrospinning were reviewed in detail by Li et al. [7].

\subsubsection{The Electrospinning Apparatus}

The properties of nanofibers that have been fabricated are broadly dependent on the initial sol-gel processing of the polymer blend (e.g., polymer concentration, polarity, solvents used), environmental conditions (humidity and temperature), and parameters used during the electrospinning process (e.g., electric field, polymer flow rate) [8,9]. In this section, the modifications that have been made to the electrospinning apparatus will be discussed. Some of the commonly used configurations are presented here.

\subsubsection{Changes in Collector Design}

The collector is where the nanofibers are deposited in the process of electrospinning. The basic electrospinning setup consists of a grounded flat surface over which the fibers are collected. Hence, in order to create specific nanostructures, the design of the collector can be modified.

\subsection{Rotating Mandrel}

The rotating mandrel collector consists of a grounded/negatively charged cylinder whose rotation is controlled through an external motor as seen in Figure 1. The use of a rotating mandrel collector helps in the fabrication of aligned nanofibers (Figure 3). The alignment of the fibers is along the direction of the rotation of the collector. The linear rotational velocity of the collector affects the morphology, mechanical strength of the fibers, and crystallinity $[10,11]$. The alignment of fibers along the rotation of the collector takes place only after reaching a critical value of rotational velocity, which is dependent on the time taken for jet solidification. The electrical field and fluid flow rate are also responsible for the alignment. A higher value of the electric field leads to breaking of the fibers while a higher flow rate of the polymer solution provides less time for the jet to solidify and align along the surface. Interestingly, high rotational speeds yield bands of fibers or overlapping fiber architectures. This was used by Persano et al. to create a three-dimensional mat, which was composed of aligned fibers [12]. 


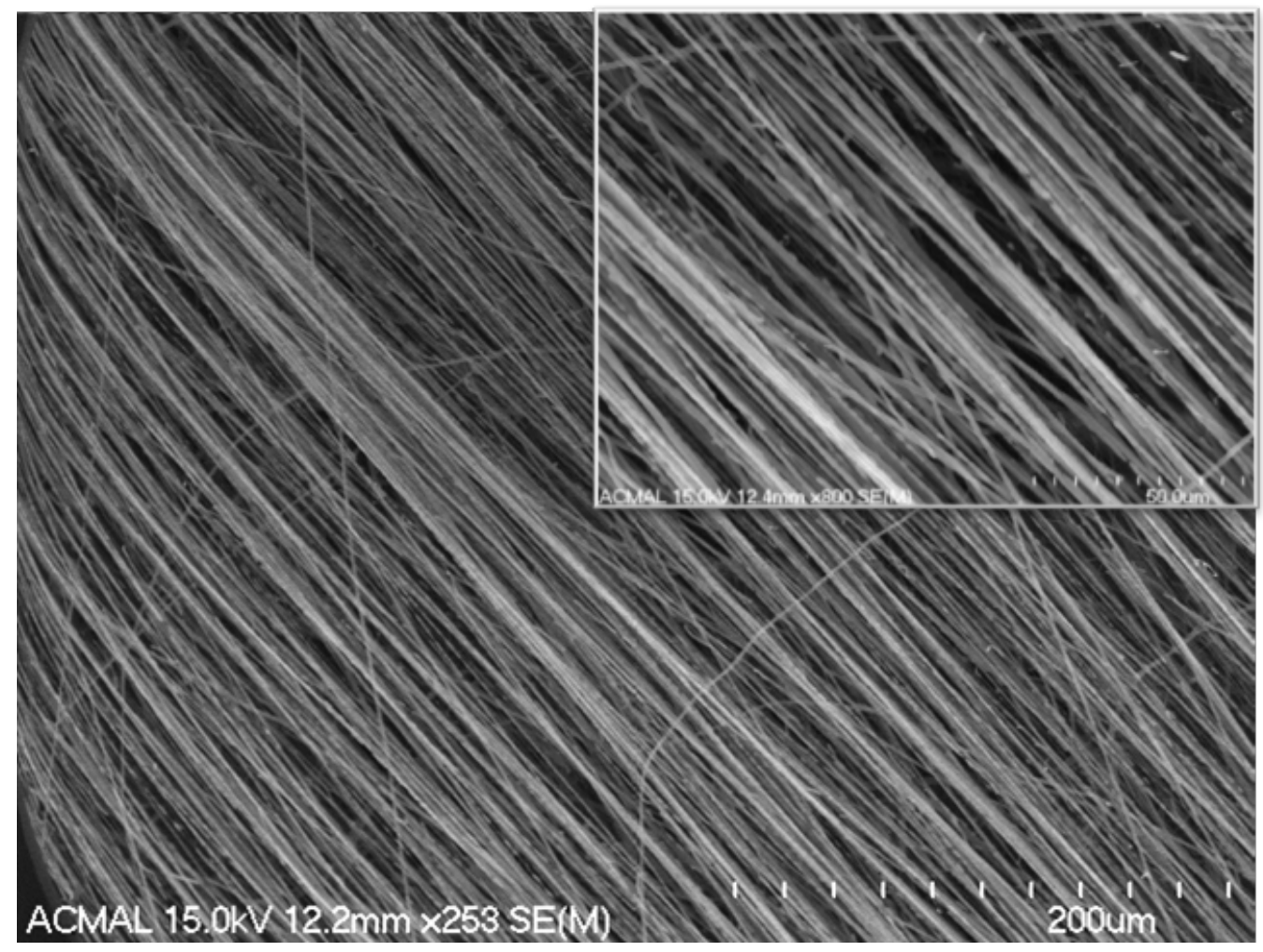

Figure 3. Field Emission Electron Scanning Microscope (FESEM) images of aligned PCL fibers fabricated on a rotating collector. The inset image is a high magnification image showing the alignment and directionality of the fibers. Adapted with permission from [18], Samerender Nagam Hanumantharao, 2017.

\subsection{Patterned Collector}

The use of a patterned collector allows the fabrication of nanofibers with varied morphologies, such as a honeycomb structure or patterns with raised morphologies (Figure 4). The collector used can be of two dissimilar materials with patterns for the selective deposition of nanofibers. The collector can be a conductive or a non-conducting surface. The fabrication of aligned nanofibers were also demonstrated by Dan Li [13]. The surface area and geometry of the collector play a major role in the deposition of fibers. Modeling the electrostatic behavior and altering the polymer solution potentially leads to the fabrication of nanofibers of novel morphologies. Ding et al. demonstrated the deposition of Poly(ethylene oxide) (PEO) fibers on a patterned collector composed of pyramidal protrusions [14]. The fibers were selected and deposited on the tips to form a pattern. 


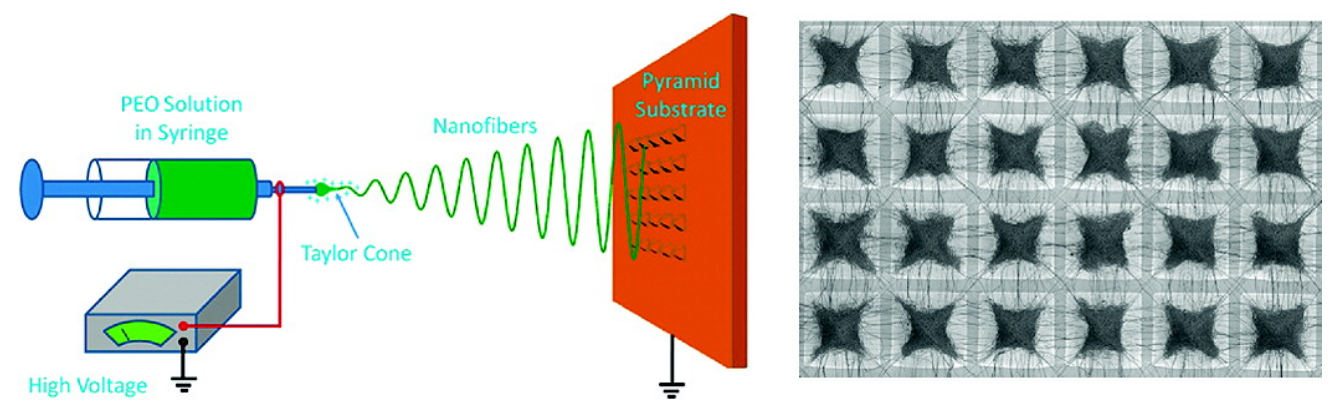

Figure 4. A representation of the electrospinning technique used by Ding and colleagues for the controlled deposition of fibers on a patterned collector. Adapted with permission from [14], American Chemical Society, 2009.

\subsection{Gap Electrospinning}

Gap electrospinning is an alternate way of achieving aligned nanofibers over long distances. A schematic representation of the gap electrospinning setup is shown in Figure 5. The collector consists of two conductive electrodes, which are separated by an insulating gap. The fibers are deposited from one end of the electrodes to the other as the insulating gap causes the fibers to depend on the electrostatic attraction provided by the conductive electrodes. The collector can further be modified with the use of multiple electrodes or stacking electrodes to get thicker 3D constructs.
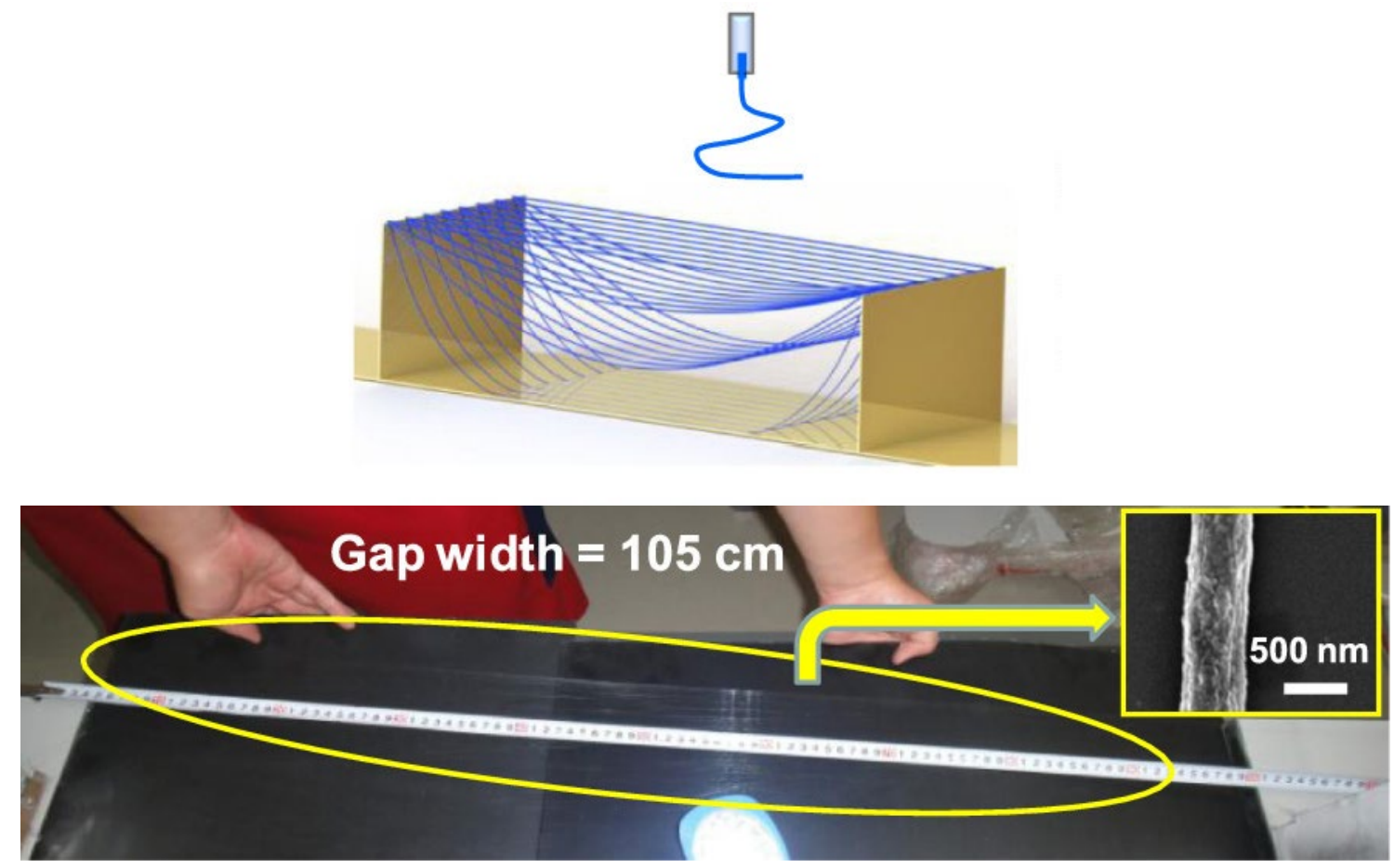
Figure 5. A representation of gap electrospinning and the length of fibers that can be produced by the technique. Adapted with permission from [36], American Chemical Society, 2018.

\subsection{Magnetic Field Associated Electrospinning}

A magnetic field has been used as a parameter to align nanofibers. Polymer solutions are deposited on a collector, which has magnets positioned in parallel, similar to gap electrospinning, to ensure alignment as reported by Yaqing Liu [37]. This technique can be used to stretch the fibers and manipulate the branching of jets to influence the electrical and mechanical properties. The technique introduces an additional force on the jet and can be used to balance out the instabilities caused in the jets.

\subsection{Wet Spinning}

In wet spinning, the electrospun fibers are collected on the surface of a liquid, such as water, which is grounded or negatively charged. In this technique, layers of random fibers are collected, leading to the fabrication of thick constructs. The use of water provides control over the porosity of the scaffold. Tzezana et al. reported the fabrication of 3D constructs for tissue engineering using hydrospinning [38]. The porosity of the scaffold fabricated ensured increased cellular infiltration compared to the fabrication of scaffolds on a plate. The porosity of the electrospun fibers was $80.4 \%$ with an average thickness of $0.29 \mathrm{~mm}$ while the fibers fabricated through wet spinning had a porosity of $99.3 \%$ with an average thickness of $10 \mathrm{~mm}$.

\subsubsection{Changes in Orientation}

This section discusses the changes that have been made in the geometrical positioning of the tip and collector. The generally used set-up is vertical electrospinning, where gravity acts on the droplet, thereby attracting it to the collector. The alternatives to these arrangements are horizontal electrospinning and converse electrospinning.

\subsection{Vertical Electrospinning}

This is the conventionally used electrospinning set-up, where the spinneret is positioned in parallel on top of a collector (Figure 6A). Gravitational forces act on the jet during electrospinning, accelerating the jet elongation and jet decay. Several studies exist to show the role of parameters and equations modeling the electrospinning process, generally considering gravitational forces to be constant throughout the process [15-18]. 


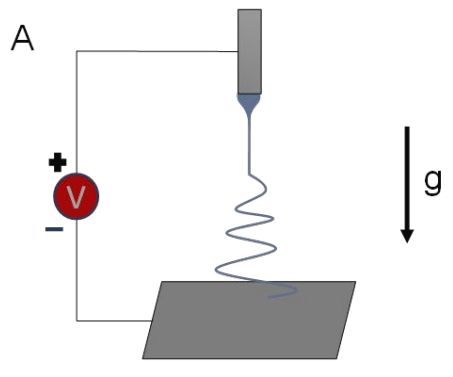

Vertical Electrospinning
B

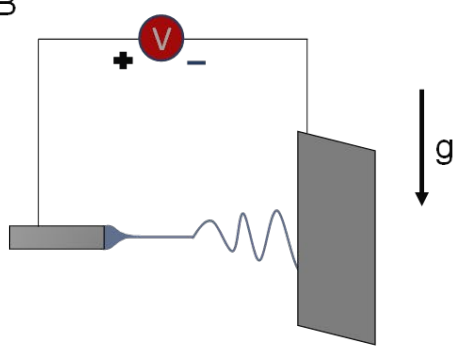

Horizontal Electrospinning

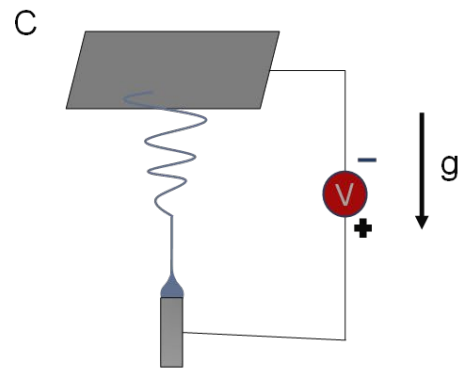

Converse Electrospinning

Figure 6. A schematic illustration of the different electrospinning modifications made by changes in orientation of the electrospinning. The effect of gravity causes changes in the resultant nanostructures and affects the electrospinning process.

\subsection{Horizontal Electrospinning}

Horizontal electrospinning is thus called because the electrical fields are parallel to the ground. The spinneret and the collector are along the same axis, parallel to the ground (Figure 6B). The gravitational forces act on the droplet in the downward direction and hence higher electrical fields are needed to overcome the surface tension of the polymer solution and gravitational forces. The projectile motion of the jet is different because of the additional forces and the altered shape of the Taylor cone.

\subsection{Converse Electrospinning}

The converse electrospinning is the converse of the vertical electrospinning method. The spinneret is positioned perpendicular to the ground underneath the collector, which is parallel to the ground (Figure 6C). The spinneret and the collector lie on the same vertical axis. The effect of gravitational force is highest on the droplet and acts against it. Hence, higher electrical fields are required to initiate the formation of a Taylor cone compared to vertical and horizontal electrospinning. Thicker diameter nanofibers with a very narrow size distribution are obtained using this technique [19].

\subsubsection{Changes in Spinneret}

\subsection{Coaxial Electrospinning}

Coaxial electrospinning is a modification where multiple polymer solutions are electrospun simultaneously from coaxial capillaries to form coaxial or multiaxial nanofibers as seen in Figure 7. The process was first demonstrated by Loscertales and colleagues in 2002, where a coaxial spinneret was used [20]. A schematic representation of the coaxial electrospinning setup and the changes in the Taylor cone during the electrospinning process are shown in Figure 3. The spinneret has two or more separate syringe pumps for the 
injection of the polymer solutions in the inner and outer capillaries. The inner capillary helps in the formation of the core polymer while the outer capillaries help in the formation of the sheath(es).

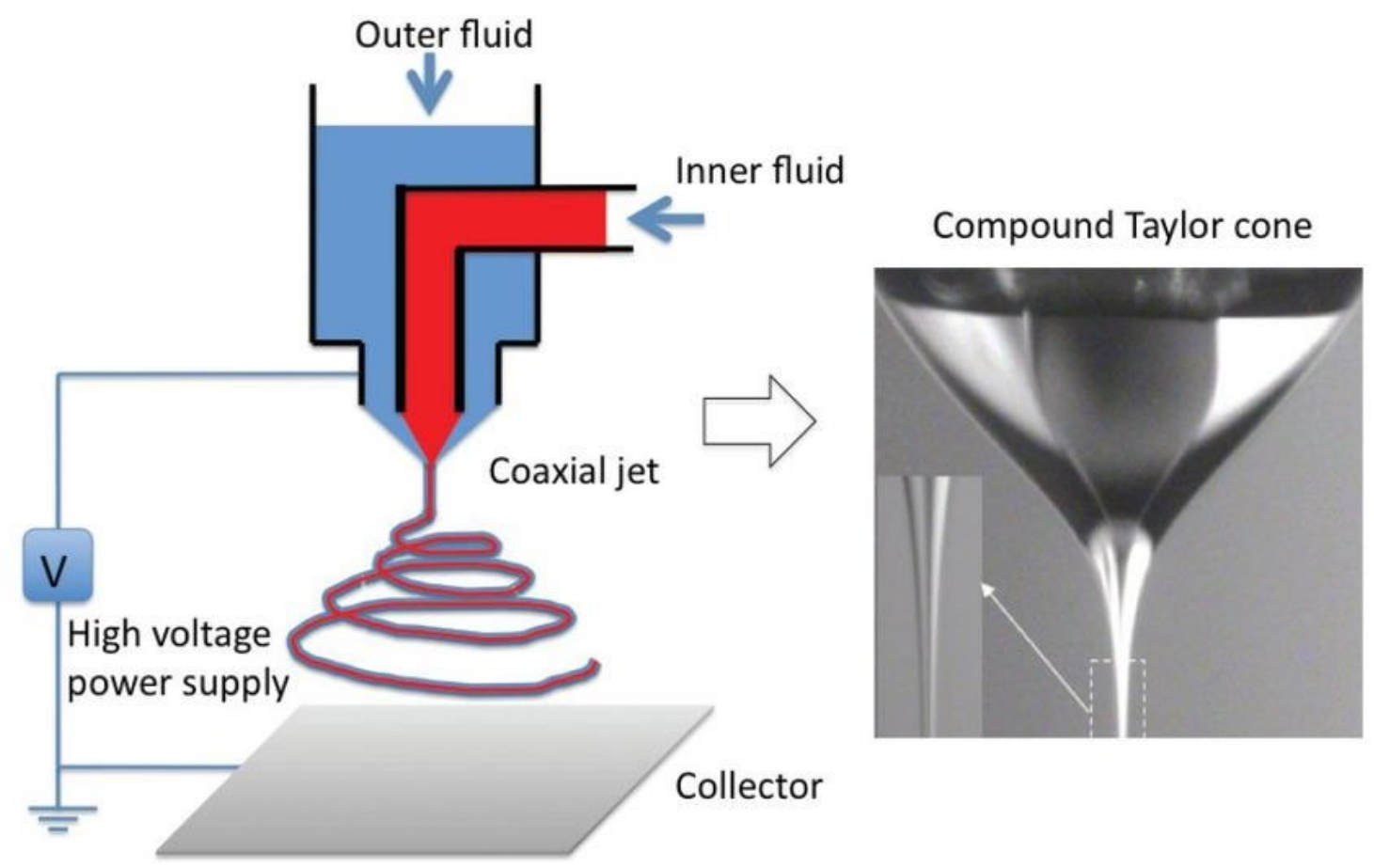

Figure 7. The basic representation of the setup for coaxial electrospinning and the change in the Taylor cone during the fabrication of common core-shell nanofibers. Reprinted with permission from [45], IntechOpen, 2010.

Interestingly, Bazilevsky and colleagues demonstrated the fabrication of coaxial fibers from a single spinneret using selective precipitation of the core polymer in a polymer blend and the polymer present on the sheath was able to form a Taylor cone along with the precipitate as seen in Figure 8 [21]. The theoretical considerations of the formation of coaxial nanofibers using a single nozzle was reviewed in detail by A.L. Yarin [22].

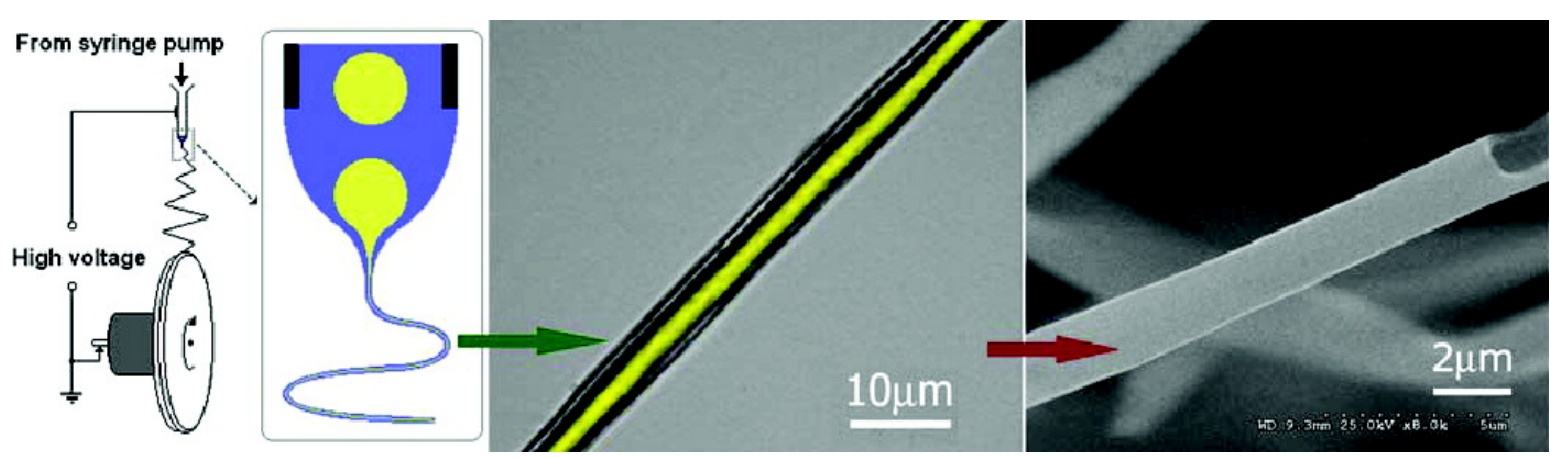


Figure 8. The setup by Bazilevsky and colleagues for the fabrication of single nozzle coaxial electrospinning of polyacrylonitrile (PAN) and poly(methyl methacrylate) (PMMA) using Dimethylformamide (DMF) as a solvent. PAN forms the core while PMMA forms the sheath of the polymer. Reprinted with permission from [21], American Chemical Society, 2007.

Coaxial electrospinning includes a few more controllable parameters than the conventional technique. Since the core and sheath polymers interact, the properties of the polymer solution, like miscibility, boiling point, and viscosity, play a significant role. Additionally, since the process involves electrospinning two or more polymer systems at the same time, the electrospinning parameters need to be compatible. The fluid flow rate is one parameter that can be individually altered during the electrospinning process. Some of the challenges during electrospinning are the solidification of jets at different points, improper elongation of the jets, and irregular flow rate ratios, which affect the continuous electrospinning process.

Recently, quadriaxial and triaxial spinnerets have also been designed and used to produce tetra-layered and triaxial nanofibers, respectively (Figure 9). The advantage of coaxial electrospinning is that it produces nanofibers of polymers with completely different properties. The core and the sheath polymers retain their properties and, in some cases, enhance the properties of the nanofibers. Also, this technique allows the electrospinning of polymer systems, which are not electrospinnable by themselves due to low viscosity or high conductivity. In order to prepare hollow nanofibers, a sacrificial polymer is used in the core, which is preferentially soluble in a solvent or more thermally degradable than the sheath polymer. Hence, coaxial electrospinning can be used to create novel polymer combinations, such as polymer/inorganic and inorganic/inorganic coaxial nanofibers, and fabrication of nanofibers that contain easily degradable compounds, like enzymes. 


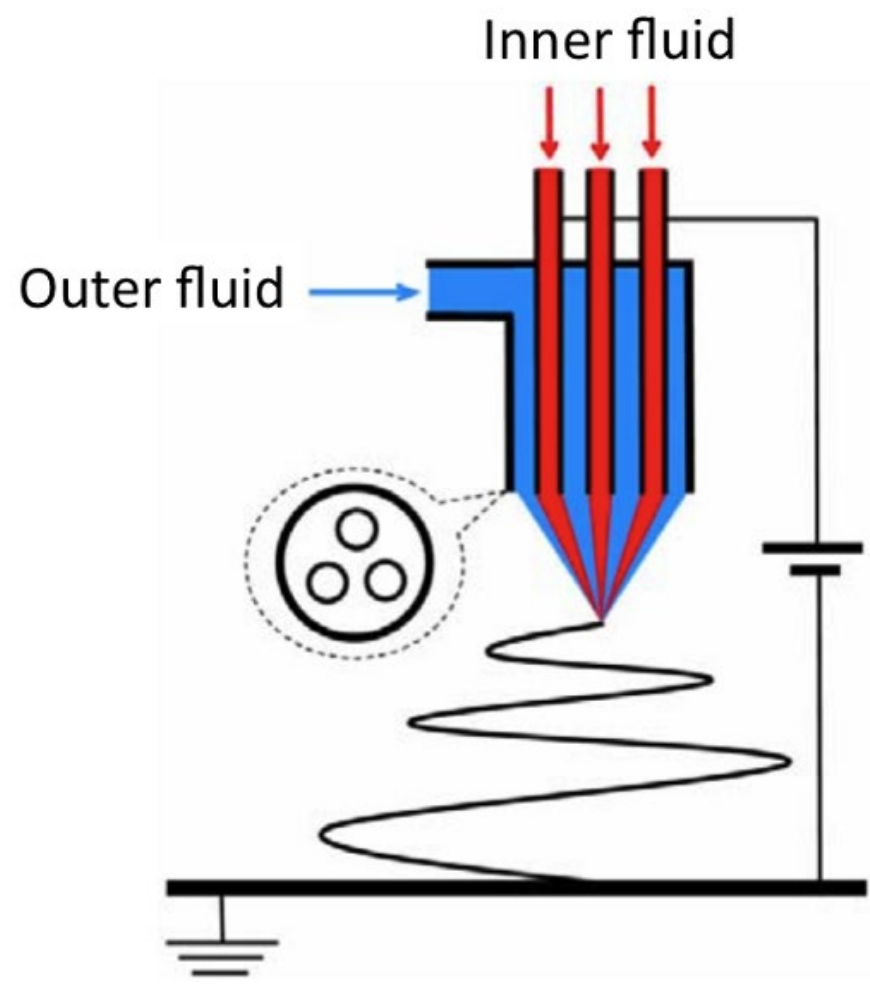

Figure 9. A schematic illustration of an example of a multiaxial spinneret system. Reprinted with permission from [45], IntechOpen, 2010.

\subsection{Co-Electrospinning}

Co-electrospinning involves the use of multiple spinnerets and simultaneous electrospinning on the same collector (Figure 10). This enables the production of composites that have the favorable properties of several polymers. Since different spinnerets are used, the morphologies of the resultant nanocomposites can be varied greatly. Modifications of this technique include using a single voltage source for multiple spinnerets or using multiple spinnerets, each with its own voltage supply. The major challenge associated with co-electrospinning is the interference of the jets during electrospinning. The charges on the jet repel each other and hence, the jets have an angle. While the parameters controlling electrospinning are applicable to co-electrospinning as well, the advantage is in the ability to prepare nanofibers on a large scale using multiple spinnerets. To ensure that there is no interference among the jets, spinnerets placed on either side of the collector to prepare aligned nanofibers have been reported [23]. Coelectrospinning is usually combined with the use of a rotating mandrel to prepare aligned nanocomposites [24]. 


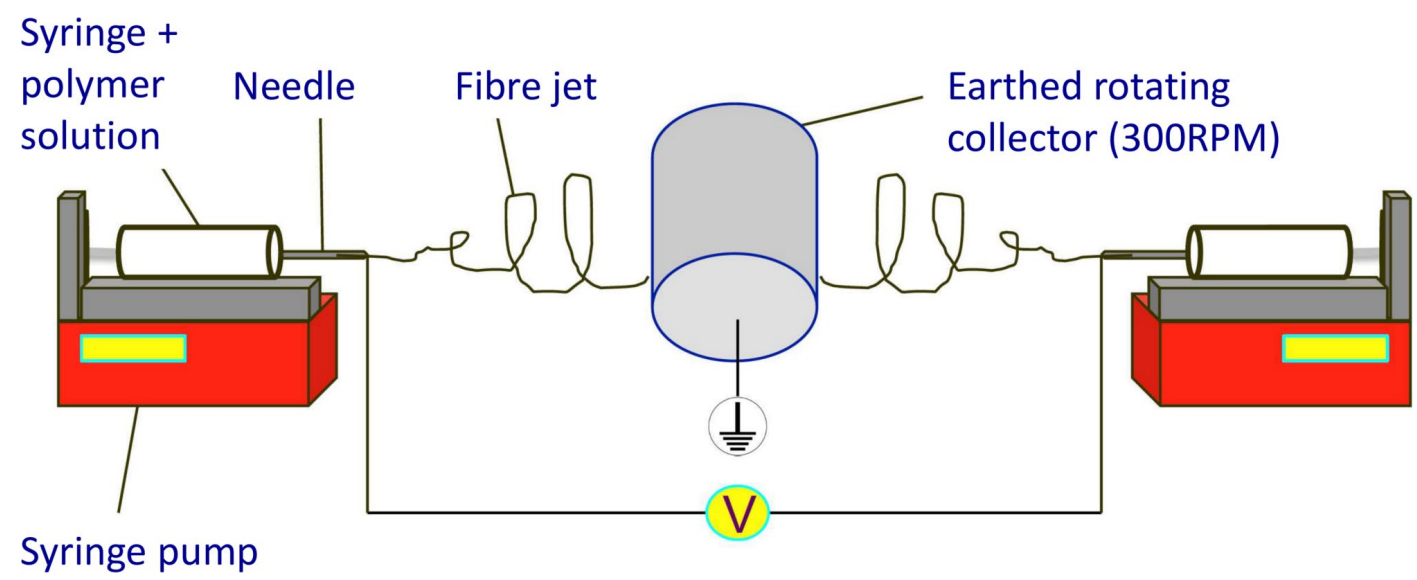

Figure 10. A graphical representation of the co-electrospinning setup used by Hillary and colleagues for the development of scaffolds with improved biophysical properties and bioactivity [50]. Reprinted with permission from [50], PLoS ONE, 2016.

\subsection{In-Line Polymer Blending}

Another approach to create nanocomposites is to combine multiple favorable properties of different polymers for use in in-line polymer blending. The polymer solutions are fed to a mixing chamber and flow through a single spinneret for electrospinning. This method of electrospinning needs precise control of the solvents and polymers used. One of the important advantages of this technique is the fabrication of nanofibers from immiscible and miscible blends, which results in novel nanomaterials.

\subsubsection{Other Modifications}

\subsection{Centrifugal Electrospinning}

Centrifugal electrospinning uses centrifugal force for the fabrication of nanofibers apart from the electrostatic and gravitational forces. Ultra-thin fibers are produced, owing to the centrifugal forces acting during jet elongation. This technique was first reported by Weitz and colleagues [25]. The set-up consists of a rotating spinneret and an annular collector, which is present around the spinneret. The diameter of the collector is the distance of separation from the tip to the collector. The revolution of the spinneret helps in providing the centrifugal force during electrospinning. This set-up can be further modified by using different spinnerets, like a coaxial spinneret, or co-electrospinning.

\subsection{Near Field Electrospinning}

The design of the setup of near field electrospinning is like the vertical electrospinning setup, except that the distance between the tip of the spinneret and the collector is in the microscale. The technique is used for the controlled deposition of fibers over a surface. 
The electric field used is high and the polymer flow rate is maintained to ensure a steady jet. Lin et al. demonstrated the use of near field electrospinning for the production of fibers with diameters ranging from 50 to $500 \mathrm{~nm}$ on a collector and the polymer feed rate was similar to that of a dip pen (Figure 11) [26].

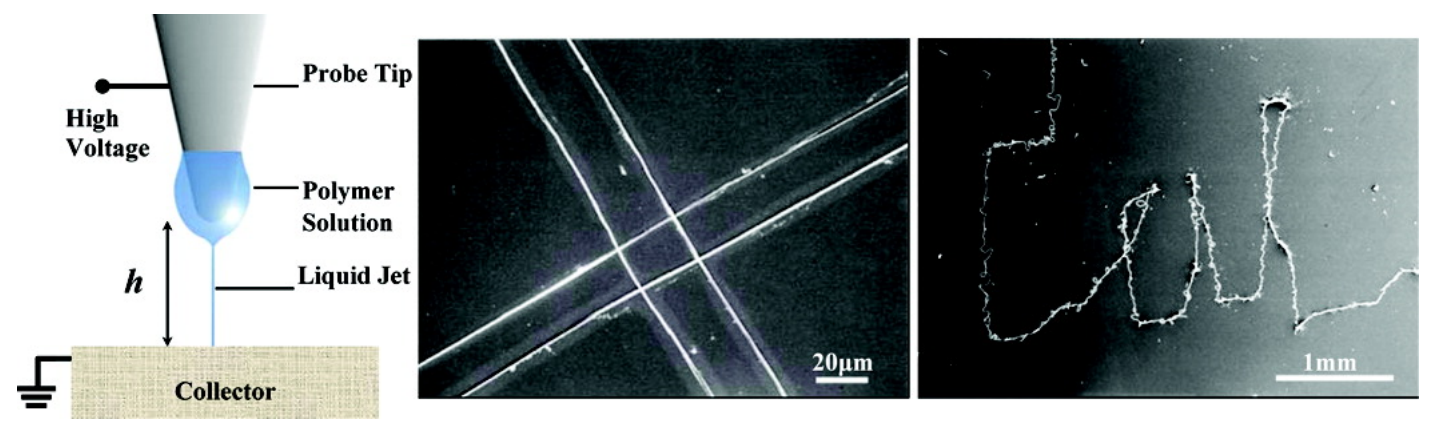

Figure 11. A schematic representation of the near field electrospinning process and the fibers obtained after electrospinning. Sun and colleagues used this technique to demonstrate the fabrication of continuous nanofibers with a desired pattern using a Tungsten tip. Reprinted with permission from [26], American Chemical Society, 2006.

\subsection{Needleless Electrospinning}

The initiation of a jet from a polymer solution can be done if the voltage applied is higher than the critical voltage needed for jet initiation. This theory was used to design spinnerets of any desired shape, a representation of which is shown in Figure 12. The first needle-less electrospinning was demonstrated and patented by Simm et al., where an annular electrode was used as the spinneret [27]. The mode of fiber generation is used to classify the two types of needle-less electrospinning setups. The rotating needle-less electrospinning setup uses mechanical forces to assist in the jet initiation from the surface of the polymer. The jets are formed from a thin layer of polymer solution on the surface of the spinneret because of the mechanical forces. This leads to perturbations assisting in the formation of Taylor cones followed by jet initiation, jet elongation, and fiber formation. Multiple jets are formed from the spinneret. The other mode of needle-less electrospinning is the stationary needle-less electrospinning, where the jet initiation occurs from the liquid surface with the use of external forces, such as magnetic fields, gravity, and the flow of gases. This technique has been in focus recently because of the ease of formation of nanofibers, higher productivity compared to conventional electrospinning, and the advantage of no clogged orifices [28]. The challenge with this technique is the use of a higher voltage for the jet initiation process. 


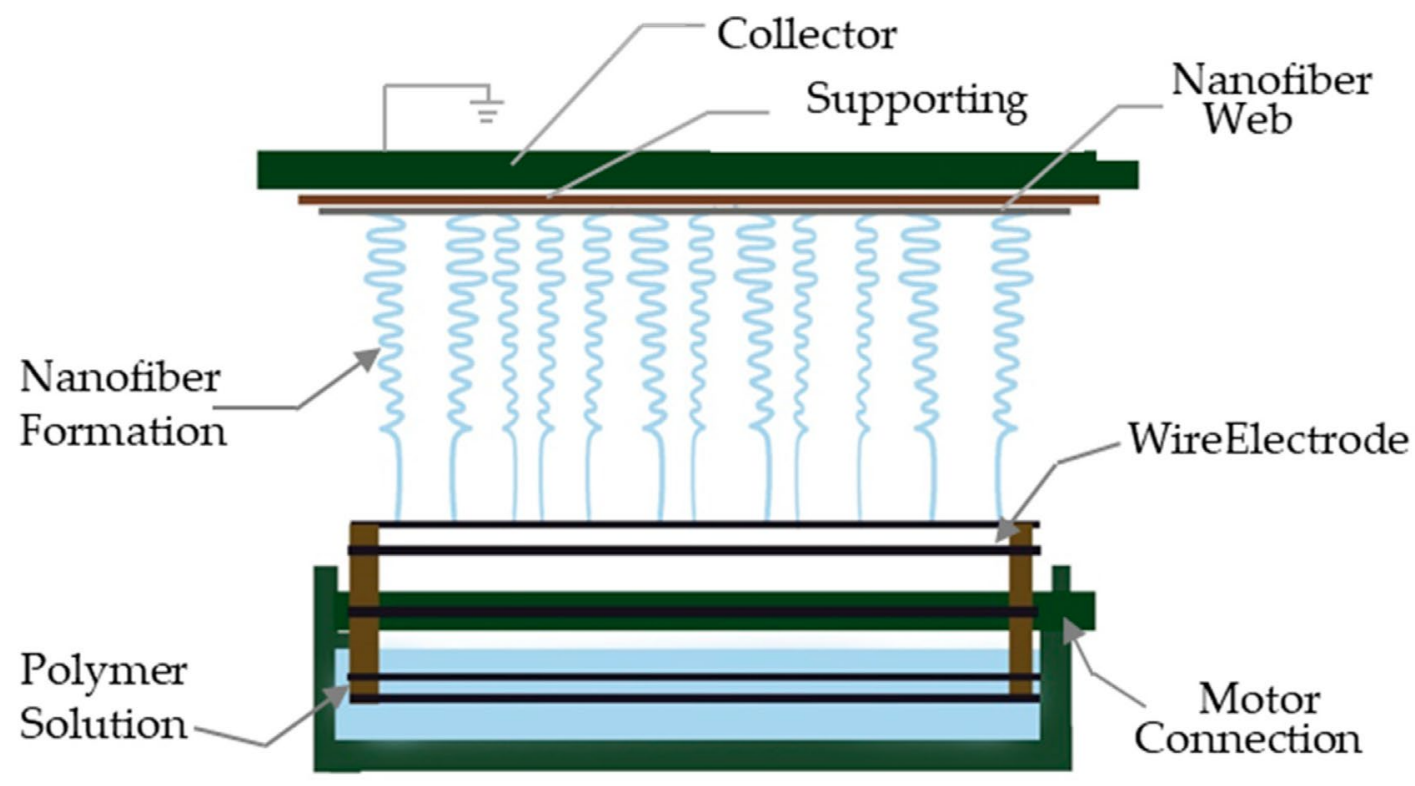

Figure 12. A basic representation of the linear needle-less electrospinning setup. Reprinted with permission from [55], MDPI, 2018.

\subsection{Emulsion Electrospinning}

Emulsion electrospinning is a technique used to create nanofibers of different compositions and structures. The feed polymer solution used for electrospinning is an emulsion of two or more polymers that are insoluble with each other. A ratio of hydrophilic and hydrophobic polymers has been used to fabricate fibers with core-shell morphologies as demonstrated by $\mathrm{Xu}$ et. al. [29]. Emulsion electrospinning is different from the single nozzle coaxial electrospinning described by Bazilevsky and colleagues [21,30]. The fibers fabricated using emulsion electrospinning may or may not have a continuous core-shell morphology but are composed of different phases of the polymers. Emulsion electrospinning provides control over the composition of the core and the sheath and diameters of the fibers by allowing modifications of the emulsion composition and the emulsification parameters. This system is beneficial in loading a drug or bioactive component that is soluble only in an inorganic solvent into an organic media or vice versa. The difference in the loading of the component is different compared to polymer blending and coaxial electrospinning as seen in Figure 13. Emulsion electrospinning is advantageous in tissue engineering applications where the bioactive components or drugs can be released in a controlled manner based on the degradation profile of the nanofibers. Some of the model proteins, microRNA, growth factors, and drugs that have been encapsulated are Bovine Serum Albumin (BSA) [31], basic fibroblasts growth factor (bFGF) [32], Cytochrome C [33], doxorubicin hydrochloride (anticancer drug) [34, 35], vascular endothelial growth factor (VEGF) and platelet-derived growth factor-bb (PDGF) [36], human-nerve growth factor [37], Rhodamine B [38], microRNA-126 [39], and epidermal 
growth factor (EGF) [40]. The other major advantage is the use of water-based solvent systems instead of more toxic solvents that provide ease of fabrication and usage.

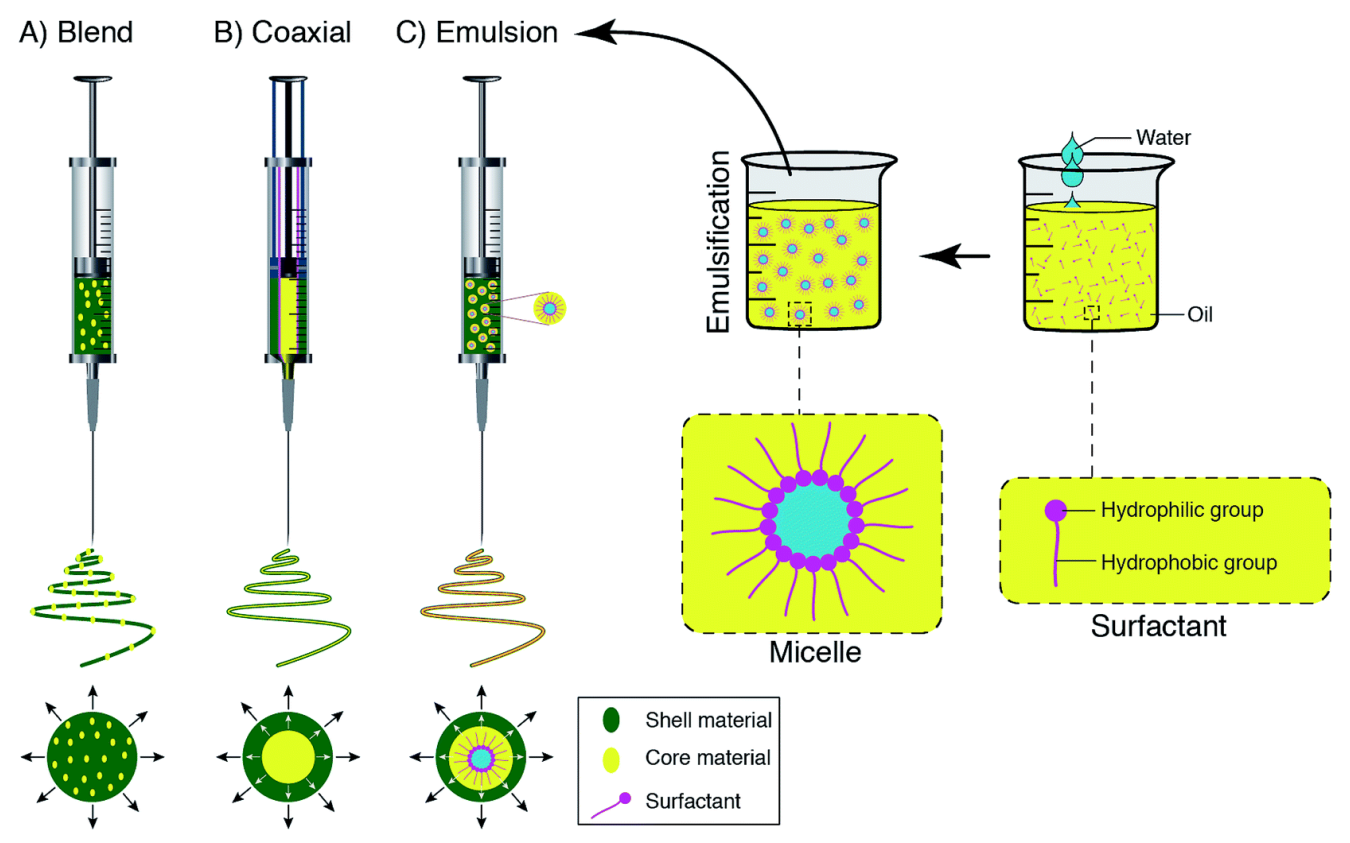

Figure 13. The difference in the loading of the polymer feed solution between blend, coaxial, and emulsion electrospinning and the difference in the resulting structure of the nanofibers. Reprinted with permission from [41], The Royal Society of Chemistry, 2017.

\subsection{Polymer Blends for Tissue Scaffold Engineering}

The blends for tissue scaffold engineering are prepared based on the requirements to mimic the extracellular microenvironment. Table 1 summarizes some of the key properties that are used to design an ideal scaffold. Apart from the key properties discussed in the table, properties, such as antibacterial activity, initiating cellular signaling, drug delivery, and electrical conductivity, are useful in scaffolds with more functionality [42-45]. The blends and functional scaffolds are designed and tested based on these design principles. Electrospinning provides flexibility for the formation of different morphologies of scaffolds. The morphologies of the scaffold play a major role in eliciting an appropriate tissue response and influences stem cell behavior [46-50]. The morphologies of the fibers include the topography of the scaffold and the diameter, orientation, and alignment of the individual nanofibers of the fibrous scaffold. 

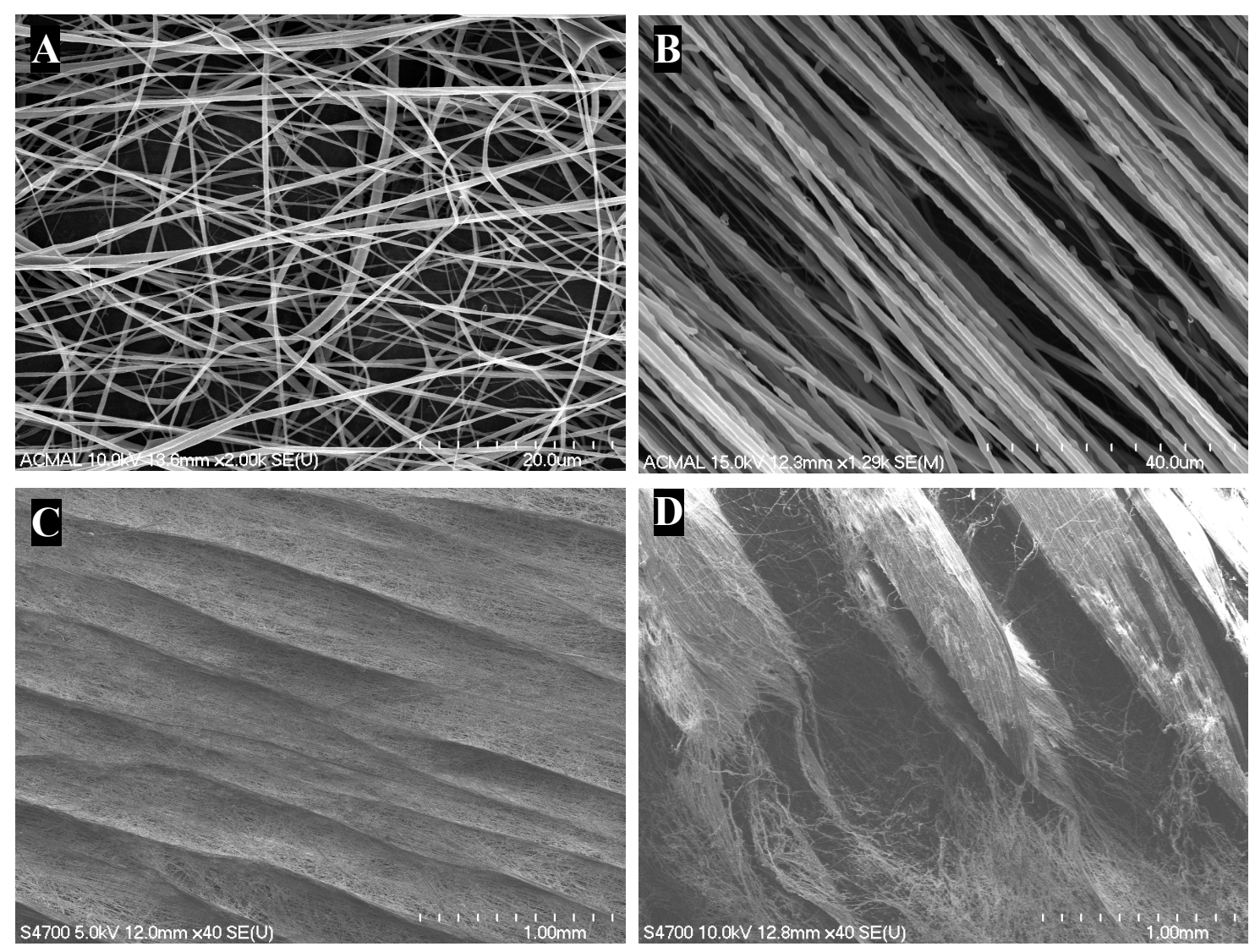

Figure 14. The field emission scanning electron microscope (FESEM) images of the commonly used morphologies for scaffold tissue engineering fabricated using electrospinning. (A) Random morphology composed of randomly oriented fibers (B) The aligned morphology consists of layers of identically oriented fibers in a $3 \mathrm{D}$ fibrous structure. (C) Overlapping morphology is composed of layers of fibers overlapped at regions (D) 3D honeycomb structured morphology consists of fibers in a specific arrangement in the scaffold.

Table 1. Properties of the scaffold considered when designing a scaffold.

Properties

Biocompatibility

Biodegradable/Nonbiodegradable

Electrical Conductivity

\section{Design Considerations}

Ensure scaffolds are compatible with the cells and do not elicit an immune response. An essential requirement of all scaffolds.

Based on the application, the scaffolds need to be biodegradable or nonbiodegradable. Biodegradable scaffolds degrade through enzymatic or hydrolytic action in a controlled manner.

Electrical signals form an integral part of the cell signaling cascade. Scaffolds that are conductive can be used to manipulate cell behavior accordingly. 


\begin{tabular}{|c|c|}
\hline Morphology & $\begin{array}{l}\text { Cell-scaffold behavior is influenced by the morphology of the scaffold. Porosity } \\
\text { is one of the properties that ensures appropriate nutrient transfer to different } \\
\text { layers of cells in the scaffold and cellular infiltration. Cellular alignment and } \\
\text { migration are also dependent on morphology. }\end{array}$ \\
\hline $\begin{array}{l}\text { Mechanical } \\
\text { Characteristics }\end{array}$ & $\begin{array}{l}\text { Mechanical properties, like the stiffness, Young's modulus, elasticity, and } \\
\text { relaxation modulus, directly affect cell behavior. Mimicking the properties of the } \\
\text { scaffold as closely as possible to the natural microenvironment is essential for an } \\
\text { ideal scaffold. }\end{array}$ \\
\hline Magnetic & $\begin{array}{l}\text { Magnetic stimulation in electroactive tissues, like cardiac, nerve, and bone } \\
\text { tissues, has shown increased cellular proliferation, differentiation, and cell } \\
\text { alignment along the direction of the magnetic field lines. The magnetic field can } \\
\text { be applied externally or by using scaffolds which exhibit magnetism. }\end{array}$ \\
\hline Bioactivity & $\begin{array}{l}\text { Bioactive scaffolds have surface ligands, like Arg-Gly-Asp (RGD) binding } \\
\text { sequences, that can be recognized by the host. They elicit a response from the } \\
\text { host due to the binding of surface receptors or peptides, or due to the release of } \\
\text { degradation products from the scaffold. }\end{array}$ \\
\hline Ease of manufacturing & $\begin{array}{l}\text { Cost of raw materials, manufacturing process, storage, etc. are some of the } \\
\text { factors that influence the effectiveness of a scaffold in tissue engineering } \\
\text { applications on a wide scale. }\end{array}$ \\
\hline
\end{tabular}

Some of the commonly used morphologies used for tissue engineering applications are scaffolds composed of randomly oriented fibers, aligned fibers, banded fibers, and honeycomb structured fibers (Figure 14). The topographies of each of these morphologies have unique properties that are beneficial in tissue engineering applications. For example, the aligned topography in certain tissues helps in better cellular migration and differentiation [51, 52]. The following sections review the developments made in the formation of functional scaffolds through polymer blending and electrospinning. The modifications in electrospinning have been used to fabricate various scaffolds with different morphologies, and structural and chemical properties that are tuned based on the intended application. The chemical properties of the scaffolds are important properties that are tuned in the case of biodegradable or bioabsorbable scaffolds, and to tune the drug release profile from the scaffold. The review by Cheng et al. provides some of the latest innovations in drug delivery from scaffolds by using electrospinning [53]. The state of the art polymer blends used for the fabrication of scaffolds for drug delivery applications were reviewed by Contreras-Cáceres and colleagues [54].

\subsubsection{Natural Polymer Blends}

Natural polymers are the first choice for the fabrication of scaffolds initially because of their biocompatibility and inherent bioactivity. The commonly used natural polymers in engineering scaffolds are components of the extracellular matrix, like collagen [55], elastin [56], hyaluronic acid [57], and fibrinogen [58], or derivatives from natural sources, like 
gelatin [59], chitosan [60,61], silk fibroin [62, 63], or vegetable oils. The main challenges associated with the use of natural polymers are the strict purification processes, chances of loss in conformation during material processing, difficulty in preparing polymer solutions, variable degradation rates, and lower mechanical strength of the scaffolds. However, polymer blending has been a useful strategy to prepare functional scaffolds that are able to overcome these common problems. The use of post-processing techniques, use of sacrificial templates, and blending with binary or ternary solvent systems has been key to the fabrication of functional scaffolds from natural polymers. Table 2 summarizes the scaffold characteristics in terms of the natural polymers used, solvents used to blend the polymers, modifications to the electrospinning equipment, tissue targeted, and a description of the research.

Table 2. Electrospun scaffolds based on natural polymer blends.

\begin{tabular}{|c|c|c|c|c|c|}
\hline Polymers Used & Solvents Used & $\begin{array}{l}\text { Type of } \\
\text { Electrospinning }\end{array}$ & $\begin{array}{l}\text { Type of } \\
\text { Tissue } \\
\text { Engineering }\end{array}$ & Comments & Ref. \\
\hline $\begin{array}{l}\text { Alginate/Gelatin } \\
\text { (PEO: sacrificial template; } \\
\text { Pluronic }{ }^{\circledR} \quad \text { F-127: } \\
\text { Surfactant) }\end{array}$ & PBS and water & $\begin{array}{l}\text { Wet } \\
\text { Electrospinning } \\
\text { (Ethanol) }\end{array}$ & $\begin{array}{l}\text { Cardiac } \\
\text { Tissue } \\
\text { Engineering }\end{array}$ & $\begin{array}{l}\text { The method of } \\
\text { polymer blending, } \\
\text { and choice of } \\
\text { electrospinning } \\
\text { helped in the } \\
\text { formation of a } \\
\text { microporous } \\
\text { network. } \\
\text { alginate/gelatin } \\
\text { hydrogel scaffolds } \\
\text { provide a } \\
\text { microenvironment } \\
\text { which help in } \\
\text { maturation of human } \\
\text { iPSC-derived } \\
\text { ventricular } \\
\text { cardiomyocyte. }\end{array}$ & {$[64]$} \\
\hline $\begin{array}{l}\text { Collagen/Chitosan/HA } \\
\text { (PEO: Sacrificial template) }\end{array}$ & $\begin{array}{l}\text { Acetic acid, DMSO } \\
\text { and water }\end{array}$ & $\begin{array}{l}\text { Vertical } \\
\text { Electrospinning }\end{array}$ & $\begin{array}{l}\text { Bone Tissue } \\
\text { Engineering }\end{array}$ & $\begin{array}{l}\text { The scaffolds } \\
\text { demonstrated } \\
\text { osteogenic } \\
\text { differentiation and } \\
\text { bone regeneration in } \\
\text { animal models. }\end{array}$ & [65] \\
\hline $\begin{array}{l}\text { Gelatin and oxidized } \\
\text { carboxymethyl cellulose }\end{array}$ & $\begin{array}{l}\text { Acetic acid and } \\
\text { water }\end{array}$ & $\begin{array}{l}\text { Rotating collector } \\
\text { coated with PEG }\end{array}$ & $\begin{array}{l}\text { Vascular } \\
\text { Tissue } \\
\text { Engineering }\end{array}$ & $\begin{array}{l}\text { Scaffolds with } \\
\text { tunable mechanical } \\
\text { properties and pore } \\
\text { sizes were fabricated. } \\
\text { Ther tubular } \\
\text { constructs (scaffolds) } \\
\text { had a homogenous } \\
\text { distribution of fibers. }\end{array}$ & [66] \\
\hline
\end{tabular}




\begin{tabular}{|c|c|c|c|c|c|}
\hline $\begin{array}{l}\text { Gelatin and } \\
\text { Bladder Matrix }\end{array}$ & 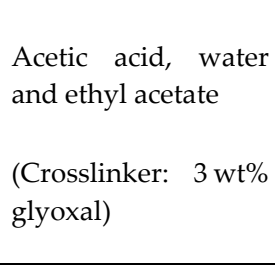 & $\begin{array}{l}\text { Low Voltage } \\
\text { Electrospinning }\end{array}$ & - & $\begin{array}{l}\text { Biofunctional ECM } \\
\text { fibers were fabricated } \\
\text { with tunable } \\
\text { biochemical, } \\
\text { mechanical, and } \\
\text { topographical } \\
\text { properties. }\end{array}$ & [67] \\
\hline Gelatin/Chitosan & $\begin{array}{l}\text { TFA and DCM (v/v } \\
7: 3)\end{array}$ & $\begin{array}{l}\text { Vertical } \\
\text { Electrospinning }\end{array}$ & $\begin{array}{l}\text { Skin Tissue } \\
\text { Engineering }\end{array}$ & $\begin{array}{l}\text { Fibrous scaffolds } \\
\text { with improved } \\
\text { mechanical } \\
\text { properties helped in } \\
\text { attachment, and } \\
\text { migration, cells } \\
\text { proliferation of } \\
\text { in vitro. }\end{array}$ & [68] \\
\hline Gelatin/Sodium Alginate & $\begin{array}{l}\text { Water } \\
\left.\text { ( } \mathrm{CaCl}_{2} \text { : crosslinker }\right)\end{array}$ & $\begin{array}{l}\text { Patterned } \\
\text { electrospinning }\end{array}$ & - & $\begin{array}{l}\text { 3D printing, freeze } \\
\text { drying, and } \\
\text { electrospinning were } \\
\text { used to manufacture } \\
\text { the porous scaffold. } \\
\text { Long term in vivo } \\
\text { studies demonstrated } \\
\text { the ability of cells to } \\
\text { vascularize on the } \\
\text { scaffolds. }\end{array}$ & [69] \\
\hline $\begin{array}{l}\text { Gelatin/Glycosaminoglyca } \\
\mathrm{n}\end{array}$ & TFE and water & Rotating collector & $\begin{array}{l}\text { Cartilage } \\
\text { Tissue } \\
\text { Engineering }\end{array}$ & $\begin{array}{l}\text { A nanofibrous } \\
\text { scaffold was } \\
\text { fabricated and tested } \\
\text { with stem cells, } 15 \% \\
\text { glycosaminoglycan in } \\
\text { gelatin matrix } \\
\text { showed the best } \\
\text { results. }\end{array}$ & [70] \\
\hline $\begin{array}{l}\text { HA and collagen } \\
\text { (PVP: sacrificial template) }\end{array}$ & Ethanol & $\begin{array}{l}\text { Vertical } \\
\text { Electrospinning }\end{array}$ & $\begin{array}{l}\text { Bone Tissue } \\
\text { Engineering }\end{array}$ & $\begin{array}{l}\text { Bottom-up method } \\
\text { was used to fabricate } \\
\text { bone Haversian } \\
\text { microstructure } \\
\text { scaffold. }\end{array}$ & [71] \\
\hline $\begin{array}{l}\text { SF and HA } \\
\text { (PEO: Sacrificial template) }\end{array}$ & Water & $\begin{array}{l}\text { Wet } \\
\text { Electrospinning }\end{array}$ & $\begin{array}{l}\text { Bone Tissue } \\
\text { Engineering }\end{array}$ & $\begin{array}{l}\text { Mussel inspired } \\
\text { polydopamine was } \\
\text { used as an adhesive } \\
\text { to coat another layer } \\
\text { of HA on the fibers } \\
\text { post-electrospinning. } \\
\text { The scaffolds } \\
\text { promoted cellular } \\
\text { differentiation in } \\
\text { vitro. }\end{array}$ & [72] \\
\hline
\end{tabular}




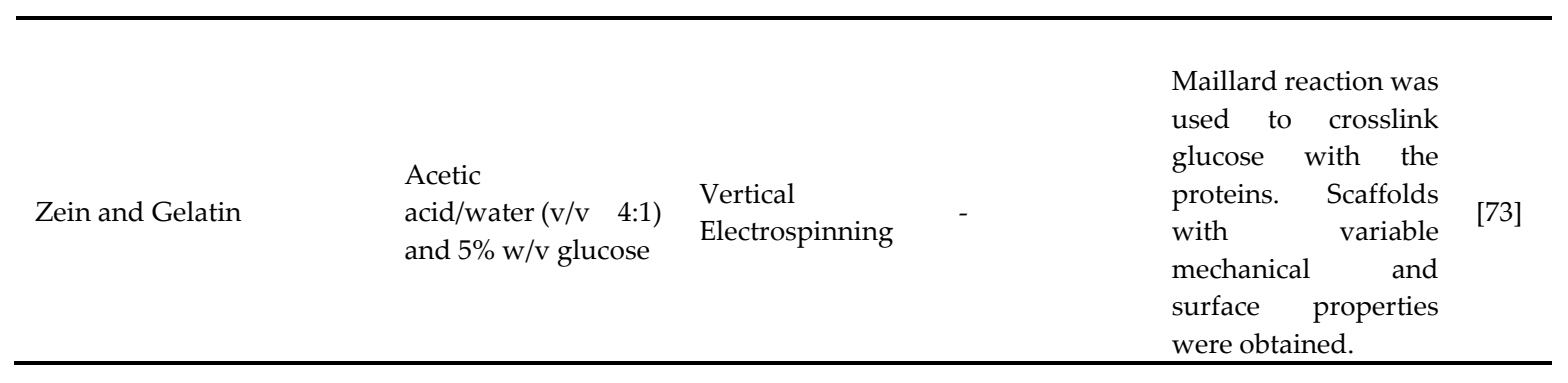

\subsubsection{Synthetic Polymer Blends}

There are a vast number of synthetic polymers that can be used for the fabrication of functional scaffolds. Polymer blending facilitates the incorporation of complementary properties of different synthetic polymers. There are several synthetic polymers that have been approved by the Food and Drug Administration (FDA) for tissue engineering, like PCL [74, 75], PLLA, PGA, and PLGA. Some of these polymers are biodegradable while some of the polymers are bioactive because of the degradation products which are recognized by the native tissues. Conductive polymers, such as PANI and PPy [76], and piezoelectric polymers [77], like PVDF, provide additional features to the scaffold and can be used to elicit electrical or mechanical stimulus to the tissues, respectively. The major advantages associated with synthetic polymers are the ease of scaling up, electrospinnability, relative affordability, superior electrical and mechanical characteristics, and relative ease of altering structures. Hanumantharao et al. demonstrated the fabrication of different morphologies of scaffolds from a polymer blend of PCL-PANI using electrospinning as seen in Figure 15. The fibroblasts used for in vitro analysis exhibited a difference in behavior based on morphology [78]. The disadvantage is that following electrospinning, the fibers need to be surface treated before culturing with cells or implantation. Table 3 summarizes the scaffold characteristics in terms of the synthetic polymers used, solvents used to blend the polymers, modifications to the electrospinning equipment, tissue targeted, and a description of the research. The most commonly used polymers as seen from the table are PCL [79], PLLA, and PPy [80]. 

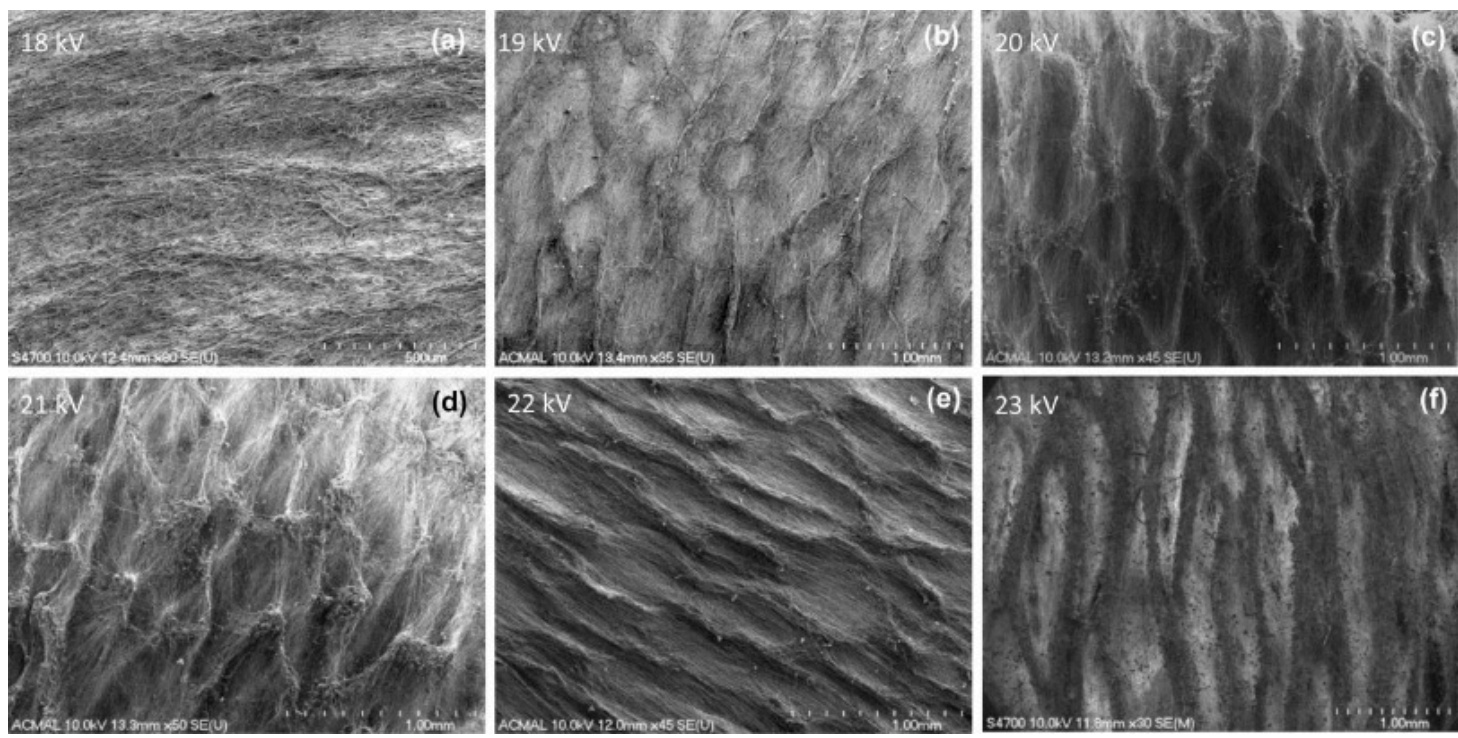

Figure 15. Field Emission Scanning Electron Microscope (FESEM) image of the different morphologies obtained using a synthetic polymer blend of PCL and PANI at different voltages. The scaffolds had no significant change in surface chemistry but had different mechanical properties. Reprinted with permission from [104], Elsevier, 2019.

Table 3. Electrospun scaffolds based on synthetic polymer blends.

\begin{tabular}{|c|c|c|c|c|c|}
\hline Polymers Used & $\begin{array}{l}\text { Solvents } \\
\text { Used }\end{array}$ & $\begin{array}{l}\text { Type of } \\
\text { Electrospinning }\end{array}$ & $\begin{array}{l}\text { Type of } \\
\text { Tissue } \\
\text { Engineering }\end{array}$ & Comments & Ref. \\
\hline PANI, PEG, and PLA & $\begin{array}{l}\text { Chloroform, } \\
\text { acetone and } \\
\text { water }\end{array}$ & $\begin{array}{l}\text { Coaxial and } \\
\text { uniaxial } \\
\text { electrospinning }\end{array}$ & $\begin{array}{l}\text { Cardiac } \\
\text { tissue } \\
\text { engineering }\end{array}$ & $\begin{array}{l}\text { Presence of doped PANI } \\
\text { and PEG helps in } \\
\text { increasing electrical } \\
\text { conductivity and affects } \\
\text { thermal properties. Use } \\
\text { of PLA helps in reducing } \\
\text { the toxicity caused by } \\
\text { PANI. }\end{array}$ & {$[81]$} \\
\hline PBAT and PPy & DMF and CF & $\begin{array}{l}\text { Climate } \\
\text { controlled } \\
\text { electrospinning }\end{array}$ & $\begin{array}{l}\text { Bone tissue } \\
\text { engineering }\end{array}$ & $\begin{array}{l}\text { The fabricated scaffolds } \\
\text { provided a surface for } \\
\text { depositing } \\
\text { nanohydroxyapatite } \\
\text { (nHAp). The scaffolds } \\
\text { were bioactive and } \\
\text { helped in the } \\
\text { differentiation of cells. }\end{array}$ & {$[82]$} \\
\hline
\end{tabular}




\begin{tabular}{|c|c|c|c|c|c|}
\hline PBAT/PPy & DMF and CF & $\begin{array}{l}\text { Vertical } \\
\text { Electrospinning }\end{array}$ & $\begin{array}{l}\text { Neural } \\
\text { Tissue } \\
\text { Engineering }\end{array}$ & $\begin{array}{l}\text { Scaffolds composed of a } \\
\text { conductive polymer } \\
\text { (PPy) and biodegradable } \\
\text { commercial polymer } \\
\text { (PBAT) were fabricated } \\
\text { through polymer } \\
\text { blending and } \\
\text { electrospinning. The } \\
\text { scaffolds supported } \\
\text { neuronal differentiation } \\
\text { and spreading. }\end{array}$ & [83] \\
\hline $\begin{array}{l}\text { PCL } \\
\text { (PVA: sacrificial template) }\end{array}$ & $\begin{array}{l}\text { PCL: CF } \\
\text { PVA: PC12 } \\
\text { cell culture } \\
\text { medium }\end{array}$ & $\begin{array}{l}\text { Liquid-liquid } \\
\text { coflowing } \\
\text { electrospinning } \\
\text { method }\end{array}$ & $\begin{array}{l}\text { Neural } \\
\text { Tissue } \\
\text { Engineering }\end{array}$ & $\begin{array}{l}\text { Fibers with PCL sheath } \\
\text { and PVA/PC12 cell cores } \\
\text { were fabricated. Cells } \\
\text { were grown inside the } \\
\text { hollow fibers after } \\
\text { dissolving the PVA. The } \\
\text { scaffold provides a route } \\
\text { to make nerve } \\
\text { connections. }\end{array}$ & [84] \\
\hline PCL and PANI & HFIP & $\begin{array}{l}\text { Vertical } \\
\text { Electrospinning }\end{array}$ & $\begin{array}{l}\text { Cardiac } \\
\text { Tissue } \\
\text { engineering }\end{array}$ & $\begin{array}{l}\text { The fabricated scaffolds } \\
\text { provide a conductive } 3 \mathrm{D} \\
\text { environment that } \\
\text { showed potential as bio } \\
\text { actuators. }\end{array}$ & [85] \\
\hline PCL and PANI & Chloroform & $\begin{array}{l}\text { Rotating } \\
\text { collector }\end{array}$ & $\begin{array}{l}\text { Skin Tissue } \\
\text { Engineering }\end{array}$ & $\begin{array}{l}\text { Honeycomb patterns of } \\
\text { varying dimensions } \\
\text { were fabricated through } \\
\text { self-assembly by altering } \\
\text { the voltage applied } \\
\text { during electrospinning. }\end{array}$ & [78] \\
\hline PCL and PGS & $\begin{array}{l}\mathrm{CF} \text { and } \\
\text { acetone }\end{array}$ & $\begin{array}{l}\text { Sequential } \\
\text { Electrospinning }\end{array}$ & $\begin{array}{l}\text { Vascular } \\
\text { tissue } \\
\text { engineering }\end{array}$ & $\begin{array}{l}\text { Tubular scaffolds were } \\
\text { fabricated from PGS and } \\
\text { PCL. The PGS (inner } \\
\text { layer) is a fast degrading } \\
\text { polymer that provides a } \\
\text { non-thrombogenic } \\
\text { surface while PCL (outer } \\
\text { layer) provides } \\
\text { mechanical stability and } \\
\text { controls the degradation } \\
\text { rate. }\end{array}$ & [86] \\
\hline
\end{tabular}




\begin{tabular}{|c|c|c|c|c|c|}
\hline PCL and PPy & $\begin{array}{l}\text { DCM/DMF } \\
(\mathrm{v} / \mathrm{v} 1: 1)\end{array}$ & $\begin{array}{l}\text { Rotating } \\
\text { collector }\end{array}$ & $\begin{array}{l}\text { Muscle } \\
\text { tissue } \\
\text { engineering }\end{array}$ & $\begin{array}{l}\text { Copolymer of PCL-PPy } \\
\text { was initially prepared } \\
\text { before electrospinning. } \\
\text { The scaffolds were } \\
\text { conductive and } \\
\text { composed of aligned } \\
\text { fibers. It was found that } \\
\text { conductivity did not } \\
\text { play a major role in } \\
\text { cellular differentiation. }\end{array}$ & [87] \\
\hline $\begin{array}{l}\text { PCL or PLLA and } \\
\text { hexaaminocyclotriphosphazene } \\
(\mathrm{HACTP})\end{array}$ & $\begin{array}{l}\text { PCL: Formic } \\
\text { acid and } \\
\text { Acetic acid } \\
\text { PLLA: TFA }\end{array}$ & $\begin{array}{l}\text { Needle-less } \\
\text { Electrospinning }\end{array}$ & - & $\begin{array}{l}\text { Two different types of } \\
\text { scaffolds were } \\
\text { fabricated. The addition } \\
\text { of HACTP increased the } \\
\text { cell spreading, } \\
\text { metabolism, } \\
\text { proliferation, and } \\
\text { bioactivity of the } \\
\text { scaffolds. }\end{array}$ & [88] \\
\hline PCL/PHB/58S bioactive glass & $\begin{array}{l}\text { CF /DMF } \\
(v / v \text { 8:2) and } \\
\text { ethanol }\end{array}$ & $\begin{array}{l}\text { Horizontal } \\
\text { electrospinning }\end{array}$ & $\begin{array}{l}\text { Skeletal } \\
\text { tissue } \\
\text { engineering }\end{array}$ & $\begin{array}{l}\text { The fabricated fibers } \\
\text { exhibited high stiffness } \\
\text { of PHB, flexibility of } \\
\text { PCL, and bioactivity of } \\
58 \mathrm{~S} \text { bioactive glass. }\end{array}$ & [89] \\
\hline PCL/PLGA and BMP-2 & $\begin{array}{l}\text { PLGA and } \\
\text { PCL: TFE } \\
\text { BMP-2: BSA } \\
\text { and water }\end{array}$ & $\begin{array}{l}\text { Coaxial } \\
\text { electrospinning }\end{array}$ & $\begin{array}{l}\text { Bone Tissue } \\
\text { Engineering }\end{array}$ & $\begin{array}{l}\text { 3D scaffolds were } \\
\text { prepared using TISA } \\
\text { post-electrospinning. } \\
\text { The scaffolds promoted } \\
\text { osteogenic } \\
\begin{array}{l}\text { differentiation and } \\
\text { proliferation. }\end{array}\end{array}$ & [90] \\
\hline PCL/PLGA/PANI & $\begin{array}{l}\text { CF/DMF } \\
\text { (v/v 3:2) }\end{array}$ & $\begin{array}{l}\text { Rotating } \\
\text { collector }\end{array}$ & $\begin{array}{l}\text { Neural } \\
\text { Tissue } \\
\text { Engineering }\end{array}$ & $\begin{array}{l}\text { Electrically conductive } \\
\text { scaffolds were } \\
\text { fabricated. The scaffolds } \\
\text { when electrically } \\
\text { stimulated resulted in } \\
\text { neurite outgrowth and } \\
\text { cell proliferation in } \\
\text { vitro. }\end{array}$ & [91] \\
\hline
\end{tabular}




\begin{tabular}{|c|c|c|c|c|c|}
\hline PCL-PLA (4:1) & $\begin{array}{l}\mathrm{DCM} / \mathrm{DMF} \\
(\mathrm{v} / \mathrm{v} 3: 2)\end{array}$ & $\begin{array}{l}\text { Rotating } \\
\text { collector }\end{array}$ & $\begin{array}{l}\text { Bone tissue } \\
\text { engineering }\end{array}$ & 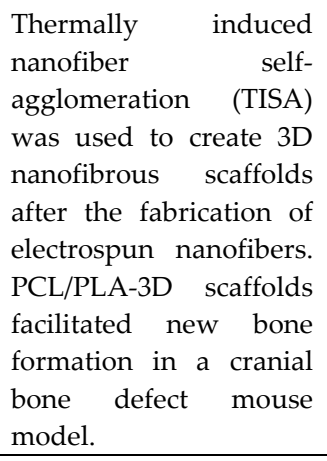 & [92] \\
\hline $\mathrm{PHBV} / \mathrm{PEO}$ & TFE & $\begin{array}{l}\text { Rotating } \\
\text { collector } \\
\text { electrospinning }\end{array}$ & $\begin{array}{l}\text { Neural } \\
\text { Tissue } \\
\text { Engineering }\end{array}$ & $\begin{array}{l}\text { The aligned PHBV/PEO } \\
\text { fibers after } \\
\text { electrospinning were } \\
\text { coated with laminin } \\
\text { after treatment with } \\
\text { plasma. The scaffolds } \\
\text { provided topographic } \\
\text { cues for the cellular } \\
\text { alignment and } \\
\text { orientation. In vivo } \\
\text { studies demonstrated } \\
\text { the effectiveness of the } \\
\text { scaffold for peripheral } \\
\text { nerve regeneration. }\end{array}$ & [93] \\
\hline \multirow[t]{2}{*}{ PU and PGS } & $\begin{array}{l}\text { Two types of } \\
\text { solvent } \\
\text { systems } \\
\text { were used. } \\
\text { CF/DMF } \\
\text { (v/v 3:2). }\end{array}$ & \multirow[t]{2}{*}{$\begin{array}{l}\text { Vertical } \\
\text { Electrospinning }\end{array}$} & \multirow[t]{2}{*}{$\begin{array}{l}\text { Vocal fold } \\
\text { tissue } \\
\text { engineering }\end{array}$} & \multirow{2}{*}{$\begin{array}{l}\text { Two different solvent } \\
\text { systems were used to } \\
\text { obtain scaffolds } \\
\text { composed of PU/PGS. } \\
\text { The morphology and } \\
\text { mechanical properties } \\
\text { were different when the } \\
\text { solvent system was } \\
\text { changed. Scaffolds } \\
\text { mimicking mechanical } \\
\text { properties of vocal folds } \\
\text { were fabricated. }\end{array}$} & \multirow[t]{2}{*}[94]{} \\
\hline & $\begin{array}{l}\text { HFIP, } \\
\text { and acetic } \\
\text { acid }\end{array}$ & & & & \\
\hline PVA and tetraethyl orthosilicate & Water & $\begin{array}{l}\text { Vertical } \\
\text { Electrospinning }\end{array}$ & - & $\begin{array}{l}\text { A 3D silica sponge was } \\
\text { fabricated using self- } \\
\text { assembly. The scaffolds } \\
\text { have high porosity, low } \\
\text { density, and } \\
\text { demonstrated high cell } \\
\text { vitality and proliferation } \\
\text { rates. }\end{array}$ & [95] \\
\hline
\end{tabular}

\subsubsection{Mixed Polymer Blends}

Mixed polymer blends are composed of a mixture of natural and synthetic polymers to form a homogenous blend, which is electrospun to fabricate functional scaffolds. The 
blending and miscibility of synthetic polymers are well characterized because of their relatively known or characterizable glass transition temperatures. Natural polymers have variable glass transition temperatures owing to the variability in processing and changes in conformations. Hence, a blend of natural and synthetic polymer is challenging. A suitable solvent system is needed for preparing the blend, such that the natural polymer does not lose its native structure and consequently, its properties in the blend. Table 4 summarizes the scaffold characteristics in terms of the natural polymers used, synthetic polymers used, solvents used to blend the polymers, modifications to the electrospinning equipment, tissue targeted, and key points of the research.

Table 4. Electrospun scaffolds based on mixture of synthetic and natural polymer blends.

\begin{tabular}{|c|c|c|c|c|c|c|}
\hline $\begin{array}{l}\text { Natural } \\
\text { Polymers }\end{array}$ & $\begin{array}{l}\text { Synthetic } \\
\text { Polymers }\end{array}$ & Solvents Used & $\begin{array}{l}\text { Type of } \\
\text { Electrospinning }\end{array}$ & $\begin{array}{l}\text { Type of } \\
\text { Tissue } \\
\text { Engineering }\end{array}$ & Comments & Ref. \\
\hline
\end{tabular}

\begin{tabular}{|c|c|c|c|c|c|c|}
\hline $\begin{array}{l}\text { 6-O- } \\
\text { Tritylchitosan } \\
\text { (Chitosan } \\
\text { derivative) }\end{array}$ & PCL & DMF & $\begin{array}{l}\text { Vertical } \\
\text { Electrospinning }\end{array}$ & $\begin{array}{l}\text { Bone Tissue } \\
\text { Engineering }\end{array}$ & $\begin{array}{l}\text { The use of chitosan } \\
\text { derivative along with } \\
\text { PCL helped in } \\
\text { increasing the } \\
\text { biocompatibility and } \\
\text { mechanical } \\
\text { properties of the } \\
\text { scaffold. }\end{array}$ & [96] \\
\hline Alginate/PEO & PCL/PEO & DMSO & $\begin{array}{l}\text { Co- } \\
\text { electrospinning }\end{array}$ & $\begin{array}{l}\text { Cancer } \\
\text { research }\end{array}$ & $\begin{array}{l}\text { Scaffolds with } \\
\text { tunable properties } \\
\text { were obtained, which } \\
\text { interacted } \\
\begin{array}{l}\text { cancer with } \\
\text { differently. }\end{array}\end{array}$ & [97] \\
\hline CA & PVP & $\begin{array}{l}\text { Acetone and } \\
\text { water }\end{array}$ & $\begin{array}{l}\text { Vertical } \\
\text { Electrospinning }\end{array}$ & $\begin{array}{l}\text { Bone Tissue } \\
\text { Engineering }\end{array}$ & $\begin{array}{l}\text { Polymer blending } \\
\text { and electrospinning } \\
\text { was used to create } \\
\text { coaxial nanofibers of } \\
\text { CA/PVP. }\end{array}$ & [98] \\
\hline $\begin{array}{l}\text { Carboxymeth } \\
\text { yl chitosan }\end{array}$ & PCL & $\begin{array}{l}\text { Acetic } \\
\text { acid/formic } \\
\text { acid (v/v 2:3) }\end{array}$ & $\begin{array}{l}\text { Vertical } \\
\text { Electrospinning }\end{array}$ & $\begin{array}{l}\text { Bone Tissue } \\
\text { Engineering }\end{array}$ & $\begin{array}{l}\text { Carboxymethyl } \\
\text { chitosan was used in } \\
\text { place of chitosan to } \\
\text { ensure the fabrication } \\
\text { of scaffolds with a } \\
\text { uniform } \\
\text { morphology. The } \\
\text { scaffolds promoted } \\
\text { cellular proliferation } \\
\text { when compared with } \\
\text { chitosan/PCL } \\
\text { scaffold. }\end{array}$ & [99] \\
\hline
\end{tabular}




\begin{tabular}{|c|c|c|c|c|c|c|}
\hline Chitosan & $\begin{array}{l}\text { Polyamide } \\
6,6\end{array}$ & $\begin{array}{l}\text { Acetic acid and } \\
\text { HFIP }\end{array}$ & $\begin{array}{l}\text { Vertical } \\
\text { Electrospinning }\end{array}$ & $\begin{array}{l}\text { Bone tissue } \\
\text { engineering }\end{array}$ & $\begin{array}{l}\text { The increase in } \\
\text { concentration of } \\
\text { chitosan showed } \\
\text { enhanced suitability } \\
\text { as scaffolds by } \\
\text { increasing the } \\
\text { bioactivity. }\end{array}$ & [100] \\
\hline Chitosan & PHB & TFA & $\begin{array}{l}\text { Vertical } \\
\text { Electrospinning }\end{array}$ & $\begin{array}{l}\text { Cartilage } \\
\text { tissue } \\
\text { engineering }\end{array}$ & $\begin{array}{l}\text { The blend was } \\
\text { prepared to increase } \\
\text { the hydrophilicity of } \\
\text { the scaffolds. }\end{array}$ & [101] \\
\hline Chitosan & PVA & Acetic acid & $\begin{array}{l}\text { Needle-less } \\
\text { Electrospinning }\end{array}$ & - & $\begin{array}{l}\text { The scaffolds have a } \\
\text { controlled } \\
\text { degradation rate and } \\
\text { mechanical } \\
\text { properties. }\end{array}$ & [102] \\
\hline Chitosan & PCL & $\begin{array}{l}\text { DCM and DMF } \\
(\mathrm{v} / \mathrm{v} 7: 3)\end{array}$ & $\begin{array}{l}\text { Vertical } \\
\text { Electrospinning }\end{array}$ & - & 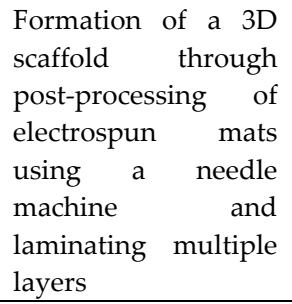 & [103] \\
\hline Chitosan & PCL & $\mathrm{DMF}$ and $\mathrm{CF}$ & Rotating collector & - & $\begin{array}{l}\text { Nano fibrillated } \\
\text { chitosan was blended } \\
\text { with PCL to } \\
\text { electrospin the } \\
\text { scaffolds, resulting in } \\
\text { improved } \\
\text { mechanical and } \\
\text { surface properties } \\
\text { compared to PCL. }\end{array}$ & [104] \\
\hline Chitosan & PVA & $\begin{array}{l}\text { Acetic acid and } \\
\text { water }\end{array}$ & $\begin{array}{l}\text { Vertical } \\
\text { Electrospinning }\end{array}$ & - & $\begin{array}{l}\text { The nanofibers were } \\
\text { crosslinked with } \\
\text { glutaraldehyde post } \\
\text { electrospinning. The } \\
\text { mechanical } \\
\text { properties of the } \\
\text { scaffolds could be } \\
\text { varied by changing } \\
\text { the crosslinking time. }\end{array}$ & [105] \\
\hline Chitosan & PLA & $\begin{array}{l}\text { PLA: CF } \\
\text { Chitosan: acetic } \\
\text { acid }\end{array}$ & $\begin{array}{l}\text { Vertical } \\
\text { Electrospinning }\end{array}$ & - & $\begin{array}{l}\text { A porous nanofiber } \\
\text { network of fibers was } \\
\text { fabricated using a } \\
\text { binary } \\
\text { system. }\end{array}$ & [106] \\
\hline
\end{tabular}




\begin{tabular}{|c|c|c|c|c|c|c|}
\hline $\begin{array}{l}\text { Chitosan and } \\
\text { hyaluronic } \\
\text { acid }\end{array}$ & $\begin{array}{l}\text { PCL } \\
\text { (PEO: } \\
\text { Sacrificial } \\
\text { Template) }\end{array}$ & $\begin{array}{l}\text { Water, Formic } \\
\text { Acid, Acetones }\end{array}$ & $\begin{array}{l}\text { Vertical } \\
\text { Electrospinning }\end{array}$ & $\begin{array}{l}\text { Skin Tissue } \\
\text { Engineering }\end{array}$ & $\begin{array}{l}\text { A 3D bilayered } \\
\text { scaffold composed of } \\
\text { chitosan/PCL- } \\
\text { hyaluronic acid was } \\
\text { fabricated. The } \\
\text { scaffold showed } \\
\text { good mechanical and } \\
\text { surface properties as } \\
\text { well as facilitated cell } \\
\text { proliferation and } \\
\text { nutrient transfer in } \\
\text { comparison to PCL } \\
\text { and chitosan/PCL. }\end{array}$ & [107] \\
\hline Collagen & $\begin{array}{l}\text { PCL and } \\
\text { nano } \\
\text { bioglass }\end{array}$ & Acetic acid & $\begin{array}{l}\text { Vertical } \\
\text { Electrospinning }\end{array}$ & $\begin{array}{l}\text { Nerve Tissue } \\
\text { engineering }\end{array}$ & $\begin{array}{l}\text { Bioactive with } \\
\text { tunable } \\
\text { biodegradation rates } \\
\text { were fabricated. }\end{array}$ & [108] \\
\hline Collagen & PLA & $\begin{array}{l}\text { HFIP and } \\
\text { Water (v/v 8:2) }\end{array}$ & $\begin{array}{l}\text { Patterned } \\
\text { Electrospinning }\end{array}$ & $\begin{array}{l}\text { Skin Tissue } \\
\text { Engineering }\end{array}$ & $\begin{array}{l}\text { Multi-level } \\
\text { architecture scaffolds } \\
\text { were obtained by } \\
\text { using a patterned } \\
\text { collector. Collagen } \\
\text { helped in improving } \\
\text { the mechanical and } \\
\text { surface properties of } \\
\text { the scaffold. }\end{array}$ & [109] \\
\hline Collagen & PLGA & HFIP & $\begin{array}{l}\text { Co- } \\
\text { electrospinning }\end{array}$ & $\begin{array}{l}\text { Neural } \\
\text { Tissue } \\
\text { Engineering }\end{array}$ & $\begin{array}{l}\text { The fabricated } \\
\text { scaffolds had the } \\
\text { advantages of } \\
\text { collagen and PLGA. } \\
\text { The scaffolds were } \\
\text { tested using TBI } \\
\text { models on animals } \\
\text { and were found to be } \\
\text { successful. }\end{array}$ & [110] \\
\hline Collagen & PCL & HFIP & $\begin{array}{l}\text { Modified } \\
\text { Electrospinning } \\
\text { setup }\end{array}$ & $\begin{array}{l}\text { Wound } \\
\text { healing } \\
\text { applications }\end{array}$ & $\begin{array}{l}\text { Manipulation of the } \\
\text { collector during } \\
\text { fabrication was used } \\
\text { to fabricate a } \\
\text { nanotopographical } \\
\text { patterned scaffold } \\
\text { with control over the } \\
\text { porosity and pore } \\
\text { size. }\end{array}$ & [111] \\
\hline Collagen & PCL & HFIP & $\begin{array}{l}\text { Near } \quad \text { Field } \\
\text { Electrospinning }\end{array}$ & - & $\begin{array}{l}\text { Near-field } \\
\text { electrospinning was } \\
\text { used to create } \\
\text { interconnected fiber } \\
\text { junctions and fiber } \\
\text { overlays. }\end{array}$ & [112] \\
\hline
\end{tabular}


HFIP

$\begin{array}{lllll}\begin{array}{l}\text { Decellularize } \\ \mathrm{d} \text { meniscus }\end{array} & \text { PCL } & \text { (Crosslinker: 1- } & \text { Horizontal } & \text { Meniscus } \\ \text { extracellular } & & \text { ethyl-3-3- } & \text { Electrospinning } & \text { tissue } \\ \text { matrix } & & \begin{array}{l}\text { dimethylamino } \\ \text { propyl } \\ \text { carbodimide) }\end{array} & \\ & & & \end{array}$

In a series of studies,

the fabrication of

decellularized

meniscus

extracellular

matrix/PCL and their

use as scaffolds for

meniscus repair were

discussed. The

scaffold had the

surface receptors

from the [113,

decellularized 114]

meniscus

extracellular matrix

and the tensile

strength from PCL.

Post-electrospinning,

freeze drying, and

crosslinking was

done to ensure the

scaffold mimicked

the natural meniscus

microenvironment.

The scaffolds were

fabricated

by

alternating between

electrospinning

PLGA and

electrospraying
PLGA: HFIP;

Demineralize

d Bone Matrix PLGA

and HA

and
Multi-jet Calvarial

electrospinning

with rotating

collector

hyaluronate:

Water defect

reconstructi

on demineralized bone matrix and HA on a

$\mathrm{Mg}$ alloy mesh. The

scaffolds provided an attractive treatment option for calvarial defect reconstruction without the use of additional growth factors.

PCL improved the mechanical properties of the scaffold while gelatin and fibrinogen increased the bioactivity and surface properties of the scaffold. Optimal concentrations of the components in polymer blend were necessary to fabricate the scaffold. 


\begin{tabular}{|c|c|c|c|c|c|c|}
\hline Gelatin & PGS-PMMA & HFP & $\begin{array}{l}\text { Vertical } \\
\text { Electrospinning }\end{array}$ & $\begin{array}{l}\text { Nerve tissue } \\
\text { engineering }\end{array}$ & $\begin{array}{l}\text { Uniform nanofibers } \\
\text { obtained from PGS- } \\
\text { PMMA/gelatin } \\
\text { blends, which were } \\
\text { biocompatible. The } \\
\text { PGS-PMMA blend } \\
\text { has tunable } \\
\text { molecular weights } \\
\text { and thermal } \\
\text { properties. }\end{array}$ & [117] \\
\hline Gelatin & PCL & $\begin{array}{l}\text { TFE and acetic } \\
\text { acid }\end{array}$ & $\begin{array}{l}\text { Vertical } \\
\text { Electrospinning }\end{array}$ & $\begin{array}{l}\text { Endotheliu } \\
\mathrm{m} \\
\text { regeneration }\end{array}$ & $\begin{array}{l}\text { Addition of gelatin } \\
\text { increased } \\
\text { hydrophilicity but } \\
\text { decreased } \\
\text { mechanical } \\
\text { properties. A balance } \\
\text { between the two was } \\
\text { shown to act as a } \\
\text { superior scaffold }\end{array}$ & [118] \\
\hline Gelatin & PCL & TFE & $\begin{array}{l}\text { Vertical } \\
\text { Electrospinning }\end{array}$ & $\begin{array}{l}\text { Vascular } \\
\text { Tissue } \\
\text { Engineering }\end{array}$ & $\begin{array}{l}\text { Human umbilical } \\
\text { vein endothelial cells } \\
\text { and adipose-derived } \\
\text { mesenchymal stem } \\
\text { cells were co- } \\
\text { cultured on the } \\
\text { PCL/gelatin scaffolds } \\
\text { to form blood vessels. }\end{array}$ & [119] \\
\hline Gelatin & $\begin{array}{l}\text { Poly(ester- } \\
\text { urethane) } \\
\text { urea }\end{array}$ & $\begin{array}{l}\text { HFIP } \\
\text { (Crosslinker: } \\
\text { Glutaraldehyd } \\
\text { e) }\end{array}$ & $\begin{array}{l}\text { Conjugated } \\
\text { electrospinning }\end{array}$ & $\begin{array}{l}\text { Skin Tissue } \\
\text { Engineering }\end{array}$ & $\begin{array}{l}\text { Nanoyarns were } \\
\text { formed using the } \\
\text { modified technique } \\
\text { of electrospinning. } \\
\text { Gelatin helped in } \\
\text { increased the } \\
\text { wettability of the } \\
\text { scaffolds. }\end{array}$ & [120] \\
\hline Gelatin & PLLA-CL & HFIP & $\begin{array}{l}\text { Conjugated } \\
\text { electrospinning } \\
\text { with rotating } \\
\text { collector }\end{array}$ & $\begin{array}{l}\text { Annulus } \\
\text { Fibrosus } \\
\text { Tissue } \\
\text { Engineering }\end{array}$ & $\begin{array}{l}\text { Aligned nanoyarn } \\
\text { scaffolds } \\
\text { fabricated, were } \\
\text { have a fibrous } \\
\begin{array}{l}\text { morphology } \\
\text { allowed and } \\
\text { infiltration } \\
\text { proliferation in vivo. }\end{array}\end{array}$ & [121] \\
\hline
\end{tabular}




\begin{tabular}{|c|c|c|c|c|c|c|}
\hline $\begin{array}{lr}\text { Gelatin } & \text { and } \\
\text { Aloe } & \text { Vera } \\
\text { extract } & \end{array}$ & PCL & Acetic acid & $\begin{array}{l}\text { Co- } \\
\text { Electrospinning }\end{array}$ & $\begin{array}{l}\text { Skin Tissue } \\
\text { Engineering }\end{array}$ & $\begin{array}{l}\text { The addition of aloe } \\
\text { vera extract to the } \\
\text { polymer blend } \\
\text { during } \\
\text { electrospinning } \\
\text { helped in increasing } \\
\text { fibroblast } \\
\text { proliferation } \\
\text { compared to PCL and } \\
\text { PCL/gelatin } \\
\text { scaffolds. }\end{array}$ & [122] \\
\hline $\begin{array}{l}\text { Gelatin and } \\
\text { Chitosan }\end{array}$ & PGS & Acetic acid & $\begin{array}{l}\text { Vertical } \\
\text { Electrospinning }\end{array}$ & $\begin{array}{l}\text { Nerve tissue } \\
\text { engineering }\end{array}$ & $\begin{array}{l}\text { PGS/chitosan/gelatin } \\
(1: 1: 2) \text { was used to } \\
\text { produce nanofibers } \\
\text { at the } 80 \mathrm{~nm} \text { scale. } \\
\text { Gelatin was } \\
\text { incorporated to make } \\
\text { ther blend } \\
\text { homogenous. }\end{array}$ & [123] \\
\hline $\begin{array}{l}\text { Gelatin and } \\
\text { Chondroitin } \\
\text { sulfate }\end{array}$ & PVA & $\begin{array}{l}\text { Acetic acid and } \\
\text { water }\end{array}$ & Rotating collector & - & $\begin{array}{l}\text { Ternary blend } \\
\text { consisting of gelatin, } \\
\text { chondroitin sulfate, } \\
\text { and PVA was used to } \\
\text { fabricate a bead free } \\
\text { nanofibrous scaffold. }\end{array}$ & [124] \\
\hline $\begin{array}{l}\text { Gelatin and } \\
\text { HA }\end{array}$ & PLLA & HFIP & $\begin{array}{l}\text { Vertical } \\
\text { Electrospinning }\end{array}$ & $\begin{array}{l}\text { Bone Tissue } \\
\text { Engineering }\end{array}$ & $\begin{array}{l}\text { Post-processing of } \\
\text { electrospun scaffolds } \\
\text { was done by } \\
\text { homogenizing, } \\
\text { freeze-drying, and } \\
\text { thermal crosslinking } \\
\text { techniques to obtain a } \\
\text { 3D scaffold. }\end{array}$ & [125] \\
\hline $\begin{array}{l}\text { Gelatin and } \\
\text { Hyaluronic } \\
\text { acid }\end{array}$ & PLA & HFIP & $\begin{array}{l}\text { Vertical } \\
\text { Electrospinning }\end{array}$ & $\begin{array}{l}\text { Cartilage } \\
\text { Tissue } \\
\text { Engineering }\end{array}$ & $\begin{array}{l}\text { A } \quad 3 D \text { scaffold } \\
\text { composed } \\
\text { gelatin/PLA } \\
\text { crosslinked with } \\
\text { hyaluronic acid was } \\
\text { fabricated, which } \\
\text { demonstrated } \\
\text { enhanced repair of } \\
\text { cartilage defects in } \\
\text { rabbits. }\end{array}$ & [126] \\
\hline
\end{tabular}




\begin{tabular}{|c|c|c|c|c|c|c|}
\hline $\begin{array}{l}\text { Gelatin } \\
\text { methacrylami } \\
\text { de }\end{array}$ & PCL & HFIP & $\begin{array}{l}\text { Horizontal } \\
\text { Electrospinning }\end{array}$ & $\begin{array}{l}\text { Vascular } \\
\text { Tissue } \\
\text { Engineering }\end{array}$ & $\begin{array}{l}\text { An optimized } \\
\text { concentration of } \\
\text { polymers in the blend } \\
\text { was obtained for } \\
\text { appropriate } \\
\text { mechanical and } \\
\text { surface properties. } \\
\text { The scaffolds } \\
\text { supported the } \\
\text { endothelial } \\
\text { remodeling cell } \\
\text { providing by } \\
\text { required biological } \\
\text { cues the } \\
\text { mechanotransductio } \\
\text { n. }\end{array}$ & [127] \\
\hline $\begin{array}{l}\text { Gelatin } \\
\text { methacrylami } \\
\text { de }\end{array}$ & PCL & $\begin{array}{l}\text { HFIP } \\
\text { (Ethanol and 2- } \\
\text { Hydroxy-4'-(2- } \\
\text { hydroxyethoxy } \\
\text { )-2- } \\
\text { methylpropiop } \\
\text { henone used } \\
\text { for } \\
\text { photocrosslinki } \\
\text { ng) }\end{array}$ & $\begin{array}{l}\text { Co- } \\
\text { Electrospinning }\end{array}$ & $\begin{array}{l}\text { Vascular } \\
\text { Tissue } \\
\text { Engineering }\end{array}$ & $\begin{array}{l}\text { A shape morphing } \\
\text { scaffold was } \\
\text { manufactured by } \\
\text { post-processing the } \\
\text { gelatin } \\
\text { methacrylamide/PCL } \\
\text { fibers and combining } \\
\text { it with a shape } \\
\text { memory polymer. } \\
\text { The scaffold was } \\
\text { rolled into 3D tube } \\
\text { structures at } \\
\text { physiological The } \\
\text { temperatures. Thaffolds provided an } \\
\text { scaffold } \\
\text { adequate } \\
\text { microenvironment } \\
\text { for inducing } \\
\text { endothelization. }\end{array}$ & [128] \\
\hline HA & PCL & $\begin{array}{l}\mathrm{DCM} / \mathrm{DMF} \\
(\mathrm{v} / \mathrm{v} 3: 2)\end{array}$ & $\begin{array}{l}\text { Patterned } \\
\text { electrospinning }\end{array}$ & $\begin{array}{l}\text { Bone Tissue } \\
\text { Engineering }\end{array}$ & $\begin{array}{l}\text { Alternating } \\
\text { electrospinning and } \\
\text { electro spraying, and } \\
\text { a honeycomb } \\
\text { patterned collector } \\
\text { were used to obtain a } \\
\text { scaffold composed of } \\
\text { multiple layers of } \\
\text { honeycomb } \\
\text { patterned PCL } \\
\text { nanofibers with HA } \\
\text { nanoparticles. In } \\
\text { vitro analysis } \\
\text { revealed the scaffold } \\
\text { promoted } \\
\text { osteocompatibility } \\
\text { and osteoconduction. }\end{array}$ & [129] \\
\hline
\end{tabular}




\begin{tabular}{|c|c|c|c|c|c|c|}
\hline $\begin{array}{l}\text { HA } \\
\text { bioceramic }\end{array}$ & $\begin{array}{l}\text { PVA and } \\
\text { PCL }\end{array}$ & $\begin{array}{l}\text { PCL and HA } \\
\text { bioceramic: CF } \\
\text { and Methanol } \\
\text { PVA: Water }\end{array}$ & $\begin{array}{l}\text { Co- } \\
\text { Electrospinning }\end{array}$ & $\begin{array}{l}\text { Bone Tissue } \\
\text { Engineering }\end{array}$ & $\begin{array}{l}\text { The favorable } \\
\text { properties of all three } \\
\text { components helped } \\
\text { in the fabrication of a } \\
\text { scaffold that } \\
\text { supported the } \\
\text { growth of stromal } \\
\text { stem cells. }\end{array}$ & [130] \\
\hline HSA & PCL & $\begin{array}{l}\text { HFIP and } \\
\text { water }\end{array}$ & Electronetting & - & $\begin{array}{l}\text { Bimodal structures } \\
\text { were obtained in the } \\
\text { shape of webs, which } \\
\text { help in cell } \\
\text { attachment. }\end{array}$ & [131] \\
\hline $\begin{array}{l}\text { Human Liver } \\
\text { ECM proteins }\end{array}$ & PLLA & $\begin{array}{l}\text { HFIP and } \\
\text { Acetic acid }\end{array}$ & $\begin{array}{l}\text { Vertical } \\
\text { Electrospinning }\end{array}$ & $\begin{array}{l}\text { Liver Tissue } \\
\text { Engineering }\end{array}$ & $\begin{array}{l}\text { A translatable niche } \\
\text { for hepatocytes } \\
\text { obtained } \\
\text { providing } \\
\text { biochemical } \\
\text { from the } \\
\text { proteins the } \\
\begin{array}{l}\text { structural } \\
\text { from PLLA. }\end{array} \\
\end{array}$ & [132] \\
\hline Lactic acid & PCL & $\begin{array}{l}\text { DCM/DMF (9:1 } \\
\text { ratio by } \\
\text { weight). }\end{array}$ & $\begin{array}{l}\text { Multiple pins } \\
\text { rotator } \\
\text { electrospinning }\end{array}$ & $\begin{array}{l}\text { Connective } \\
\text { tissues }\end{array}$ & $\begin{array}{lr}\begin{array}{l}\text { Scaffolds } \\
\text { aligned }\end{array} & \text { mimicking } \\
\text { fibrils } & \text { wellagen } \\
\text { fabricated. } & \end{array}$ & [133] \\
\hline $\begin{array}{l}\text { Laminin and } \\
\text { Collagen }\end{array}$ & $\begin{array}{l}\text { Polydioxano } \\
\text { ne }\end{array}$ & $\begin{array}{l}\text { HFIP and } \\
\text { water }\end{array}$ & $\begin{array}{l}\text { Magnetic field- } \\
\text { assisted } \\
\text { electrospinning } \\
\text { with coaxial } \\
\text { spinneret }\end{array}$ & $\begin{array}{l}\text { Neural } \\
\text { Tissue } \\
\text { Engineering }\end{array}$ & $\begin{array}{l}\text { Aligned laminin- } \\
\text { polydioxanone/colla } \\
\text { gen core-shell fibers } \\
\text { were fabricated. } \\
\text { Laminin was } \\
\text { systematically } \\
\text { released from the } \\
\text { fibers. The scaffolds } \\
\text { promoted the } \\
\text { hippocampal cell } \\
\text { behaviors in vitro. }\end{array}$ & [134] \\
\hline Lecithin & PLA and PU & $\begin{array}{l}\text { THF/DMF } \\
\text { mixture }\end{array}$ & $\begin{array}{l}\text { Horizontal } \\
\text { electrospinning } \\
\text { with rotating } \\
\text { collector }\end{array}$ & $\begin{array}{l}\text { Liver Tissue } \\
\text { Engineering }\end{array}$ & $\begin{array}{l}\text { The use of PU and } \\
\text { lecithin helped in } \\
\text { increasing the } \\
\text { flexibility, } \\
\text { hydrophilicity, and } \\
\text { bioactivity. The fibers } \\
\text { also had higher } \\
\text { hydrophilicity and } \\
\text { biocompatibility than } \\
\text { the tissue culture } \\
\text { plate. }\end{array}$ & [135] \\
\hline
\end{tabular}




\begin{tabular}{|c|c|c|c|c|c|c|}
\hline Lignin & PCL & $\mathrm{CF}$ & $\begin{array}{l}\text { Vertical } \\
\text { Electrospinning }\end{array}$ & $\begin{array}{l}\text { Neural } \\
\text { Tissue } \\
\text { Engineering }\end{array}$ & 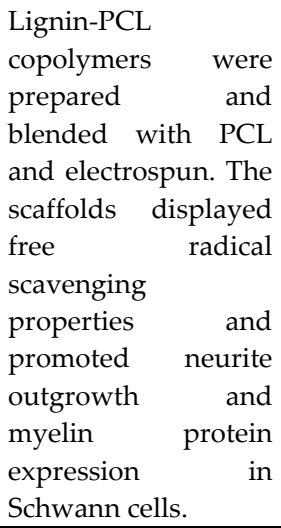 & [136] \\
\hline $\begin{array}{l}\text { Neem oil and } \\
\text { Corn oil }\end{array}$ & PU & $\mathrm{DMF}$ & $\begin{array}{l}\text { Vertical } \\
\text { Electrospinning }\end{array}$ & $\begin{array}{l}\text { Bone Tissue } \\
\text { Engineering }\end{array}$ & $\begin{array}{l}\text { Neem oil and corn oil } \\
\text { were integrated into } \\
\text { the PU matrix to } \\
\text { fabricate } \\
\text { biocompatible } \\
\text { scaffolds with a } \\
\text { higher tensile } \\
\text { strength and } \\
\text { hydrophilicity in } \\
\text { relation to PU/corn } \\
\text { oil and PU scaffolds. }\end{array}$ & [137] \\
\hline Oyster shell & PLLA & $\begin{array}{l}\text { CF/DMF } \quad(v / v \\
3: 1)\end{array}$ & Rotating collector & $\begin{array}{l}\text { Bone Tissue } \\
\text { Engineering }\end{array}$ & $\begin{array}{l}\text { The scaffolds were } \\
\text { composed of aligned } \\
\text { fibers. The scaffolds } \\
\text { promoted cellular } \\
\text { adhesion and } \\
\text { differentiation in } \\
\text { vivo. }\end{array}$ & [138] \\
\hline SF & PU & HFIP & $\begin{array}{l}\text { Vertical } \\
\text { Electrospinning }\end{array}$ & $\begin{array}{l}\text { Cardiac } \\
\text { Tissue } \\
\text { Engineering }\end{array}$ & $\begin{array}{l}\text { The scaffolds had } \\
\text { variable degradation } \\
\text { rates and mechanical } \\
\text { properties, which } \\
\text { could be controlled } \\
\text { by modifying the } \\
\text { ratio of SF in the } \\
\text { blend. The scaffolds } \\
\text { were viable } \\
\text { candidates for heart } \\
\text { valve tissue } \\
\text { engineering }\end{array}$ & [139] \\
\hline
\end{tabular}




\begin{tabular}{|c|c|c|c|c|c|c|}
\hline SF & PLGA & $\begin{array}{l}\text { PLGA: } \\
\text { and DMF } \\
\text { SF: Formic } \\
\text { Acid }\end{array}$ & $\begin{array}{l}\text { Multilayer } \\
\text { electrospinning }\end{array}$ & $\begin{array}{l}\text { Skin Tissue } \\
\text { Engineering }\end{array}$ & $\begin{array}{l}\text { A novel method of } \\
\text { electrospinning was } \\
\text { used to prepare a } \\
\text { sandwich of PLGA } \\
\text { between SF. The } \\
\text { scaffold fabricated } \\
\text { helped in the } \\
\text { proliferation of skin } \\
\text { cells. }\end{array}$ & [140] \\
\hline SF & $\begin{array}{l}\text { PEO } \\
\text { (sacrificial } \\
\text { template) }\end{array}$ & $\begin{array}{l}\text { Ethanol, Water, } \\
\text { Formic acid, } \\
\text { Calcium } \\
\text { chloride }\end{array}$ & $\begin{array}{l}\text { Jet } \\
\text { Electrospinning }\end{array}$ & - & $\begin{array}{l}\text { Highly aligned fibers } \\
\text { were produced using } \\
\text { stable jet } \\
\text { electrospinning to } \\
\text { form a scaffold with } \\
\text { high anisotropy. }\end{array}$ & [141] \\
\hline SF & PCL & Formic acid & $\begin{array}{l}\text { Wet } \\
\text { Electrospinning }\end{array}$ & $\begin{array}{l}\text { Bone Tissue } \\
\text { Engineering }\end{array}$ & $\begin{array}{l}\text { Post-processing of } \\
\text { the SF/PCL scaffolds } \\
\text { was done by } \\
\text { functionalizing with } \\
\text { polyglutamate acid } \\
\text { conjugated with } \\
\text { BMP-2 peptide. Wet } \\
\text { electrospinning } \\
\text { helped in the } \\
\text { formation of 3D } \\
\text { scaffolds. The } \\
\text { functionalized } \\
\text { scaffolds enhanced } \\
\text { cellular } \\
\text { differentiation in } \\
\text { comparison with the } \\
\text { SF/PCL scaffold. }\end{array}$ & [142] \\
\hline SF & PLLA-CL & HFIP & Rotating collector & $\begin{array}{l}\text { Bone Tissue } \\
\text { Engineering }\end{array}$ & $\begin{array}{l}\text { A dual layered } \\
\text { scaffold composed of } \\
\text { random and aligned } \\
\text { fibers was fabricated. } \\
\text { It was found to be a } \\
\text { suitable model for } \\
\text { tendon to bone } \\
\text { healing from in vivo } \\
\text { experiments. }\end{array}$ & [143] \\
\hline
\end{tabular}




\begin{tabular}{|c|c|c|c|c|c|c|}
\hline SF & PLLA-CL & HFIP & $\begin{array}{l}\text { Vertical } \\
\text { Electrospinning }\end{array}$ & $\begin{array}{l}\text { Conjunctiva } \\
\text { Reconstructi } \\
\text { on }\end{array}$ & $\begin{array}{l}\text { Transparent scaffolds } \\
\text { were fabricated, } \\
\text { which are } \\
\text { hydrophilic and } \\
\text { porous. Conjunctival } \\
\text { epithelial cells were } \\
\text { seeded on the } \\
\text { scaffolds. The cells } \\
\text { seeded on scaffolds } \\
\text { were able to form } \\
\text { stratified } \\
\text { conjunctival } \\
\text { epithelium, including } \\
\text { goblet cells }\end{array}$ & [144] \\
\hline SF & PEO & Water & $\begin{array}{l}\text { Vertical } \\
\text { Electrospinning }\end{array}$ & $\begin{array}{l}\text { Periodontal } \\
\text { tissue } \\
\text { regeneration }\end{array}$ & $\begin{array}{l}\text { Ultrasonication was } \\
\text { used as a parameter } \\
\text { to alter the viscosity } \\
\text { of the sol-gel prior to } \\
\text { electrospinning. The } \\
\text { amount of polymer in } \\
\text { the final scaffold } \\
\text { could be varied using } \\
\text { this technique. }\end{array}$ & [145] \\
\hline $\begin{array}{l}\text { SF and } \\
\text { Platelet-rich } \\
\text { plasma }\end{array}$ & $\begin{array}{l}\text { PCL and } \\
\text { PVA }\end{array}$ & $\begin{array}{l}\text { HFIP } \\
\text { water }\end{array}$ & $\begin{array}{l}\text { Co- } \\
\text { electrospinning }\end{array}$ & $\begin{array}{l}\text { Bone Tissue } \\
\text { Engineering }\end{array}$ & $\begin{array}{l}\text { Platelet rich plasma } \\
\text { was incorporated } \\
\text { into the scaffolds by } \\
\text { making a suitable } \\
\text { blend with PVA. Co- } \\
\text { electrospinning was } \\
\text { used to fabricate } \\
\text { scaffolds with SF, } \\
\text { PCL, PVA, and } \\
\text { platelet rich plasma. } \\
\text { The scaffolds } \\
\text { exhibited a sustained } \\
\text { release of platelet rich } \\
\text { plasma and } \\
\text { promoted cellular } \\
\text { differentiation, } \\
\text { proliferation, and } \\
\text { migration. }\end{array}$ & [146] \\
\hline Starch & PVA & Ethanol & $\begin{array}{l}\text { Vertical } \\
\text { Electrospinning }\end{array}$ & $\begin{array}{l}\text { Wound } \\
\text { healing } \\
\text { applications }\end{array}$ & $\begin{array}{l}\text { Crosslinking using } \\
\text { glutaraldehyde post- } \\
\text { electrospinning } \\
\text { helped in improving } \\
\text { the mechanical } \\
\text { properties of the } \\
\text { scaffold. }\end{array}$ & [147] \\
\hline
\end{tabular}




\begin{tabular}{|c|c|c|c|c|c|c|}
\hline $\begin{array}{l}\text { Sunflower oil } \\
\text { and Neem oil }\end{array}$ & PU & DMF & $\begin{array}{l}\text { Vertical } \\
\text { Electrospinning }\end{array}$ & $\begin{array}{l}\text { Bone Tissue } \\
\text { Engineering }\end{array}$ & $\begin{array}{l}\text { Plant oils were } \\
\text { successfully } \\
\text { integrated into the } \\
\text { polymer matrix to } \\
\text { enhance the } \\
\text { mechanical and } \\
\text { properties the } \\
\text { bioactivity of } \\
\text { scaffolds. }\end{array}$ & [148] \\
\hline Tussah SF & PLA & HFIP & $\begin{array}{l}\text { Double conjugate } \\
\text { electrospinning } \\
{[149]}\end{array}$ & $\begin{array}{l}\text { Bone Tissue } \\
\text { Engineering }\end{array}$ & $\begin{array}{l}\text { A novel method of } \\
\text { electrospinning was } \\
\text { used to fabricate } \\
\text { scaffolds with high } \\
\text { mechanical strength. }\end{array}$ & [150] \\
\hline $\begin{array}{l}\text { Virgin } \\
\text { coconut oil }\end{array}$ & PU & DMF & $\begin{array}{l}\text { Vertical } \\
\text { Electrospinning }\end{array}$ & $\begin{array}{l}\text { Vascular } \\
\text { Tissue } \\
\text { Engineering }\end{array}$ & $\begin{array}{l}\text { The presence of } \\
\text { virgin coconut oil in } \\
\text { the polymer matrix } \\
\text { helped in increasing } \\
\text { antithrombogenicity, } \\
\text { surface activity, and } \\
\text { mechanical } \\
\text { properties of the } \\
\text { scaffold. }\end{array}$ & [151] \\
\hline $\begin{array}{l}\text { Zein and } \\
\text { Gum Arabic }\end{array}$ & PCL & $\begin{array}{l}\text { Formic acid } \\
\text { and glacial } \\
\text { acetic acid }\end{array}$ & $\begin{array}{l}\text { Vertical } \\
\text { Electrospinning }\end{array}$ & $\begin{array}{l}\text { Skin Tissue } \\
\text { Engineering }\end{array}$ & $\begin{array}{l}\text { PCL helped in } \\
\text { improving the } \\
\text { mechanical } \\
\text { properties, zein } \\
\text { helped in moderating } \\
\text { the degradation } \\
\text { while gum arabic } \\
\text { helped in improving } \\
\text { the surface } \\
\text { properties. }\end{array}$ & [152] \\
\hline
\end{tabular}

\subsection{Nanofiller Polymer Blends}

Fillers in polymer blends help to impart specific properties to the fibers. They can be uniformly distributed in the fibers or can be present on the surface. The fillers can aid in the formation of chemical bonds, fill regions in the polymer matrix, change the orientation of polymers, electrical conductivity, and modify surface groups. Table 5 summarizes the scaffold characteristics in terms of the polymers used, fillers used, solvents used to blend the polymers, tissue targeted, and a description of the research. Some of the commonly used nanofillers are HA [153], GO [30], and ferromagnetic nanoparticles [154, 155].

Table 5. Electrospun scaffolds based on the use of nanofiller systems.

\begin{tabular}{|c|c|c|c|c|c|}
\hline Polymers Used & Filler & Solvents Used & $\begin{array}{l}\text { Type of } \\
\text { Tissue } \\
\text { Engineering }\end{array}$ & Comments & Ref. \\
\hline
\end{tabular}




\begin{tabular}{|c|c|c|c|c|c|}
\hline $\begin{array}{l}\text { 4-arm } \quad \text { PCL-(Zn- } \\
\text { curcumin } \quad \text { complex) } \\
\text { and } \\
\text { carboxymethyl } \\
\text { chitosan }\end{array}$ & GO & $\begin{array}{l}\text { DMF } \\
\text { DCM }\end{array}$ & $\begin{array}{l}\text { Bone Tissue } \\
\text { Engineering }\end{array}$ & $\begin{array}{l}\text { Core shell nanofibers were } \\
\text { fabricated composed of PCL- } \\
\text { (Zn-curcumin complex core } \\
\text { and GO-PVA-carboxymethyl } \\
\text { chitosan sheath. The scaffolds } \\
\text { showed enhanced osteogenic } \\
\text { capability and antibacterial } \\
\text { activity. }\end{array}$ & [156] \\
\hline Agarose acetate & $\begin{array}{l}\beta \text {-tricalcium } \\
\text { phosphate }\end{array}$ & $\begin{array}{l}\text { Acetic acid } \\
\text { and DMAC }\end{array}$ & $\begin{array}{l}\text { Bone Tissue } \\
\text { Engineering }\end{array}$ & $\begin{array}{l}\text { The addition of } \beta \text {-tricalcium } \\
\text { phosphate helped in increasing } \\
\text { cellular differentiation and } \\
\text { proliferation in comparison to } \\
\text { the scaffold without the filler. }\end{array}$ & [157] \\
\hline $\begin{array}{l}\text { Alginic acid sodium } \\
\text { salt/ PVA }\end{array}$ & $\begin{array}{l}\text { Graphene } \\
\text { sheets }\end{array}$ & Water & $\begin{array}{l}\text { Neural Tissue } \\
\text { Engineering }\end{array}$ & $\begin{array}{l}\text { Electrically conductive } \\
\text { scaffolds with high mechanical } \\
\text { strength were fabricated. The } \\
\text { use of filler helped in } \\
\text { increasing the mechanical } \\
\text { strength by forming strong } \\
\text { bridges with the matrix. }\end{array}$ & [158] \\
\hline Chitosan and PU, PPy & $\begin{array}{l}\text { Functionalized } \\
\text { MWCNT }\end{array}$ & TFA & $\begin{array}{l}\text { Neural Tissue } \\
\text { Engineering }\end{array}$ & $\begin{array}{l}\text { Nerve conduit was fabricated } \\
\text { using aligned fibers. Post } \\
\text { processing } \\
\text { chitosan/PU/MWCNT fibers } \\
\text { was done by sheathing with } \\
\text { PPy. }\end{array}$ & [159] \\
\hline Chitosan/PVP & GO & $\begin{array}{l}\text { Acetic acid } \\
\text { and distilled } \\
\text { water }\end{array}$ & $\begin{array}{l}\text { Skin Tissue } \\
\text { engineering }\end{array}$ & $\begin{array}{l}\text { The preparation of chitosan- } \\
\text { based blends and addition of } \\
\text { GO increased the mechanical } \\
\text { properties of the scaffold. }\end{array}$ & [160] \\
\hline PCL & $\mathrm{ZnO}$ & HFIP & $\begin{array}{l}\text { Periodontal } \\
\text { tissue } \\
\text { engineering }\end{array}$ & $\begin{array}{l}\text { In vivo testing of the scaffolds } \\
\text { demonstrated the antibacterial } \\
\text { and osteoconductive } \\
\text { properties of the fibrous } \\
\text { scaffold. }\end{array}$ & [161] \\
\hline PCL & $\begin{array}{l}\text { Nano } \\
\text { particles }\end{array}$ & TFE & $\begin{array}{l}\text { Bone Tissue } \\
\text { Engineering }\end{array}$ & $\begin{array}{l}\text { A polymer blending method to } \\
\text { increase the quantity of nano } \\
\text { HA particles were used to } \\
\text { fabricate scaffolds. }\end{array}$ & [162] \\
\hline $\begin{array}{l}\text { (PEO: } \quad \text { Sacrificial } \\
\text { template) }\end{array}$ & HA & $\begin{array}{l}\text { Acetic acid } \\
\text { and DMSO }\end{array}$ & $\begin{array}{l}\text { Tendon and } \\
\text { Ligament } \\
\text { Regeneration }\end{array}$ & $\begin{array}{l}\text { The HA particles were } \\
\text { integrated into the polymer } \\
\text { matrix for the fabrication of } \\
\text { scaffolds, which are suitable } \\
\text { for tendon and ligament } \\
\text { regeneration. The scaffolds } \\
\text { mimic the mechanical } \\
\text { properties closely. }\end{array}$ & [163] \\
\hline
\end{tabular}




\begin{tabular}{|c|c|c|c|c|c|}
\hline PCL and Gelatin & $\begin{array}{l}\text { halloysite } \\
\text { nanotubes }\end{array}$ & Acetic acid & $\begin{array}{l}\text { Wound } \\
\text { healing } \\
\text { applications }\end{array}$ & $\begin{array}{l}\text { Needle-less and free liquid } \\
\text { surface electrospinning was } \\
\text { used to fabricate uniform mats. } \\
\text { Addition of halloysite } \\
\text { nanotubes helped in increasing } \\
\text { the mechanical properties of } \\
\text { the scaffold. }\end{array}$ & [164] \\
\hline PCL/Gelatin & $\begin{array}{l}\text { Lanthanum } \\
\text { chloride }\left(\mathrm{LaCl}_{3}\right)\end{array}$ & $\begin{array}{l}\text { PCL: DCE and } \\
\text { ethanol } \\
\text { Gelatin: } \\
\text { Formic acid } \\
\text { and ethanol }\end{array}$ & $\begin{array}{l}\text { Wound } \\
\text { Healing } \\
\text { applications }\end{array}$ & $\begin{array}{l}\text { Co-electrospinning using a } \\
\text { rotating collector was used for } \\
\text { the fabrication of the scaffolds. } \\
\text { The scaffolds showed } \\
\text { comparable mechanical } \\
\text { properties to skin and showed } \\
\text { good bioactivity. }\end{array}$ & [165] \\
\hline PCL/Gelatin/Chitosan & $\begin{array}{l}\beta \text {-tricalcium } \\
\text { phosphate }\end{array}$ & $\begin{array}{lr}\text { Acetic } & \text { acid } \\
\text { and } & \text { Formic } \\
\text { acid. } & \end{array}$ & $\begin{array}{l}\text { Bone Tissue } \\
\text { Engineering }\end{array}$ & $\begin{array}{l}\text { A functional scaffold for } \\
\text { guided bone regeneration was } \\
\text { fabricated from an immiscible } \\
\text { blend. The mechanical and } \\
\text { surface properties increased } \\
\text { with the increasing } \\
\text { concentration of the filler. }\end{array}$ & [166] \\
\hline PCL-Aloe Vera & $\begin{array}{l}\text { Mg-Ferrite } \\
\text { nanoparticles }\end{array}$ & TFE & - & $\begin{array}{l}\text { Magnetic nanofibers were } \\
\text { prepared, and in vitro viability } \\
\text { was tested on fibroblasts. }\end{array}$ & [167] \\
\hline PCL-Chitosan & $\mathrm{MgO}$ & TFE and water & - & $\begin{array}{l}\text { Fibrous scaffolds with tunable } \\
\text { physical properties were } \\
\text { fabricated. }\end{array}$ & [168] \\
\hline PEA & rGO & CF and DMF & $\begin{array}{l}\text { Cardiac } \\
\text { Tissue } \\
\text { Engineering }\end{array}$ & $\begin{array}{l}\text { The nanofiller decreased the } \\
\text { voltage required for } \\
\text { electrospinning and increased } \\
\text { the electrical conductivity of } \\
\text { the scaffolds. }\end{array}$ & [169] \\
\hline PHBV & $\begin{array}{l}\text { Silicate } \\
\text { containing HA }\end{array}$ & $\mathrm{CF}$ & $\begin{array}{l}\text { Bone Tissue } \\
\text { Engineering }\end{array}$ & $\begin{array}{l}\text { The piezoelectric activity of } \\
\text { PHBV and bioactivity of } \\
\text { silicate containing HA helped } \\
\text { in cellular differentiation, } \\
\text { alignment, and proliferation of } \\
\text { cells when compared to PHBV } \\
\text { scaffolds and PCL scaffolds. }\end{array}$ & [170] \\
\hline PLA and Chitosan & $\begin{array}{l}\text { Tricalcium } \\
\text { Phosphate }\end{array}$ & TFE & - & $\begin{array}{l}\text { Cryomilling was used to } \\
\text { prepare a fine powder of the } \\
\text { polymers and filler before } \\
\text { dissolution. The scaffolds are a } \\
\text { suitable candidate for bone } \\
\text { tissue engineering application. }\end{array}$ & [171] \\
\hline
\end{tabular}




\begin{tabular}{|c|c|c|c|c|c|}
\hline PLA and PVAc & GO & $\begin{array}{l}\text { DMF, } \\
\text { Chloroform } \\
\text { and Acetic } \\
\text { acid }\end{array}$ & $\begin{array}{l}\text { Bone Tissue } \\
\text { Engineering }\end{array}$ & $\begin{array}{l}\text { The dual-electrospinning } \\
\text { technique was used to fabricate } \\
\text { triple shaped memory } \\
\text { polymers. Addition of GO } \\
\text { helped to improve the } \\
\text { properties. }\end{array}$ & [172] \\
\hline PLGA & GO & HFIP & $\begin{array}{l}\text { Skeletal } \\
\text { tissue } \\
\text { engineering }\end{array}$ & $\begin{array}{l}\text { PLGA and GO (wt. ratio 20:3) } \\
\text { were used to create 3D } \\
\text { scaffolds with increased } \\
\text { hydrophilicity. }\end{array}$ & [173] \\
\hline PLGA & $\begin{array}{l}\text { Silica } \\
\text { Nanoparticles }\end{array}$ & HFIP & $\begin{array}{l}\text { Bone Tissue } \\
\text { Engineering }\end{array}$ & $\begin{array}{l}\text { The scaffolds fabricated } \\
\text { promoted cellular } \\
\text { differentiation, migration, and } \\
\text { proliferation. The mechanical } \\
\text { properties of the scaffolds } \\
\text { increased as the silica } \\
\text { nanoparticles helped to } \\
\text { reinforce the fibers. }\end{array}$ & [174] \\
\hline PLLA & $\mathrm{Fe}_{3} \mathrm{O}_{4}$ & $\begin{array}{l}\text { DCM/DMF } \\
(4: 1 \mathrm{v} / \mathrm{v})\end{array}$ & $\begin{array}{l}\text { Bone Tissue } \\
\text { Engineering }\end{array}$ & $\begin{array}{l}\text { The scaffolds with a filler } \\
\text { helped in the better healing of } \\
\text { bone defects in animal studies } \\
\text { in comparison with neat PLLA } \\
\text { grafts. }\end{array}$ & [175] \\
\hline PLLA/Lactic acid & $\begin{array}{l}\beta \text {-tricalcium } \\
\text { phosphate }\end{array}$ & DCM/Acetone & $\begin{array}{l}\text { Bone Tissue } \\
\text { Engineering }\end{array}$ & $\begin{array}{l}\text { Low density fluffy fibrous } \\
\text { scaffolds were fabricated. The } \\
\text { lactic acid was bleached out } \\
\text { post-electrospinning from the } \\
\text { scaffolds. The scaffolds } \\
\text { promoted cellular infiltration } \\
\text { because of their morphology } \\
\text { and bioactive filler molecules. }\end{array}$ & [176] \\
\hline $\begin{array}{l}\text { Poly(3- } \\
\text { hydroxybutyrate-co-4- } \\
\text { hydroxybutyrate) }\end{array}$ & GO & $\mathrm{CF}$ & $\begin{array}{l}\text { Bone Tissue } \\
\text { Engineering }\end{array}$ & $\begin{array}{l}\text { The GO in the scaffold helped } \\
\text { in modifying the diameter of } \\
\text { fibers, positively affecting the } \\
\text { mechanical and surface } \\
\text { properties of the scaffolds and } \\
\text { enhancing cellular } \\
\text { differentiation and } \\
\text { proliferation in comparison } \\
\text { with scaffolds without the filler } \\
\text { molecules. }\end{array}$ & [177] \\
\hline PU & $\begin{array}{l}\text { Zinc Nitrate } \\
\text { hexahydrate }\end{array}$ & DMF & $\begin{array}{l}\text { Wound } \\
\text { healing } \\
\text { applications }\end{array}$ & $\begin{array}{l}\text { The incorporation of zinc } \\
\text { nitrate in the PU scaffolds } \\
\text { helped in increasing the } \\
\text { bioavailability and blood } \\
\text { compatibility. }\end{array}$ & [178] \\
\hline
\end{tabular}




\begin{tabular}{|c|c|c|c|c|c|}
\hline PU and PDMS & $\begin{array}{l}\text { HA } \\
\text { nanoparticles }\end{array}$ & THF & $\begin{array}{l}\text { Bone Tissue } \\
\text { Engineering }\end{array}$ & $\begin{array}{l}\text { Scaffolds composed of an } \\
\text { interconnected pore network } \\
\text { were fabricated. The } \\
\text { composition of the HA } \\
\text { nanoparticles was optimized to } \\
\text { ensure maximum cell } \\
\text { proliferation and vitality. }\end{array}$ & [179] \\
\hline PVA & $\begin{array}{l}\text { Nanohydroxy } \\
\text { apatite and } \\
\text { cellulose } \\
\text { nanofibers }\end{array}$ & Water & $\begin{array}{l}\text { Bone Tissue } \\
\text { Engineering }\end{array}$ & $\begin{array}{l}\text { The fillers were used to } \\
\text { improve the mechanical } \\
\text { properties of the scaffold, } \\
\text { reduce the degradation rate, } \\
\text { and increase cellular activity in } \\
\text { relation to PVA/nanohydroxy } \\
\text { apatite and PVA fibers. }\end{array}$ & [180] \\
\hline PVA & $\gamma-\mathrm{Fe} 2 \mathrm{O} 3$ & Water & - & $\begin{array}{l}\text { The fabrication process } \\
\text { involved 3D printing and } \\
\text { thermal inversion phase } \\
\text { separation for fabrication of } \\
\text { the collector and } \\
\text { electrospinning of the polymer } \\
\text { blend with filler for obtaining } \\
\text { the scaffold. The scaffold had } \\
\text { milli, micro, and microporous } \\
\text { layers because of the } \\
\text { fabrication process. The filler } \\
\text { helped in increasing the } \\
\text { mechanical properties of the } \\
\text { scaffold in relation with PVA. }\end{array}$ & [181] \\
\hline PVA and Alginate & $\begin{array}{l}\text { Graphene }(1 \% \\
\text { PVP dispersing } \\
\text { agent })\end{array}$ & Water & - & $\begin{array}{l}\text { Needle-less electrospinning } \\
\text { was used for the fabrication of } \\
\text { conductive scaffolds with a } \\
\text { high surface area. The } \\
\text { inclusion of a filler improved } \\
\text { the properties of the scaffold } \\
\text { greatly. }\end{array}$ & [182] \\
\hline PVDF & $\begin{array}{l}\text { Barium } \\
\text { Titanate and } \\
\text { multiwalled- } \\
\text { carbon } \\
\text { nanotubes }\end{array}$ & $\begin{array}{l}\text { DMF and } \\
\text { Acetone }\end{array}$ & - & $\begin{array}{l}\text { A fluffy } 3 \mathrm{D} \text { fibrous } \\
\text { piezoelectric scaffold was } \\
\text { fabricated by controlling the } \\
\text { relative humidity during } \\
\text { electrospinning }\end{array}$ & [183] \\
\hline PVDF & GO & $\begin{array}{l}\text { DMAC and } \\
\text { Acetone }\end{array}$ & $\begin{array}{l}\text { Bone Tissue } \\
\text { Engineering }\end{array}$ & $\begin{array}{l}\text { The PVDF containing GO } \\
\text { exhibited good } \\
\text { osteoconductive properties } \\
\text { and can be used as a } \\
\text { bioimplant. }\end{array}$ & [184] \\
\hline
\end{tabular}




\begin{tabular}{|c|c|c|c|c|c|}
\hline SF & Reduced GO & Formic acid & - & $\begin{array}{l}\text { The incorporation of reduced } \\
\text { GO in the SF matrix helped } \\
\text { improve the mechanical and } \\
\text { thermal properties of the } \\
\text { scaffold. The scaffolds also } \\
\text { promoted osteogenic } \\
\text { differentiation in vitro. }\end{array}$ & [185] \\
\hline SF & $\begin{array}{l}\mathrm{CoFe}_{2} \mathrm{O}_{4} \text { and } \\
\mathrm{Fe}_{3} \mathrm{O}_{4}\end{array}$ & Formic acid & - & $\begin{array}{l}\text { Magnetic fillers were used to } \\
\text { prepare scaffolds, which are } \\
\text { magnetically responsive and } \\
\text { biodegradable. }\end{array}$ & [186] \\
\hline SF & GO & Formic acid & $\begin{array}{l}\text { Wound } \\
\text { dressing } \\
\text { applications }\end{array}$ & $\begin{array}{l}\text { Scaffolds exhibiting } \\
\text { antibacterial activity and high } \\
\text { porosity were fabricated. GO } \\
\text { was integrated into the } \\
\text { polymer matrix and this } \\
\text { increased the number of } \\
\text { oxygen containing groups. }\end{array}$ & [187] \\
\hline
\end{tabular}

\subsection{Perspectives and Conclusions}

Electrospinning is a relatively old technique but has not yet lost its significance because of its ease of use and ability to be combined with other techniques. The modifications made to the electrospinning apparatus help in adapting the process to fabricate scaffolds with a single polymer or several blended polymers or through multiple inputs. The use of polymer blends adds more flexibility to the manufacturing process. Blends between natural and synthetic polymers and the use of nanofiller systems have been used to demonstrate the fabrication of mechanical, surface, biochemical, and electrical properties, which are impossible to obtain through any single polymer. The blending and electrospinning process has also been used to prepare nanostructures from immiscible blends, which are otherwise unable to be processed. The fabrication of scaffolds that mimic the mechanical, surface, electrical, and biochemical properties of a variety of tissues have been electrospun and processed. The post-processing of the nanofibers after electrospinning by using techniques such as freeze fracture or cell electrospinning, are interesting methods for further exploration of the potential of electrospinning. Further research is needed to study the dependence on nanotopographical cues in cell behavior in vivo. Techniques, such as rotating collector electrospinning [188] and near field electrospinning [189], have been used previously to fabricate scaffolds that provide topographical cues to the cells in in vitro conditions. New inroads have also been made in the integration of nanofillers and bioactive compounds in the polymer matrix that can induce differentiation, modulate cell behavior, and prevent bacterial infections. The major challenges in the preparation of polymer blends lie in the identification of a suitable solvent system, processing conditions, and method of electrospinning. This is critical for blends containing synthetic and natural polymers, where the window for electrospinning is reduced and the prediction of solubility and miscibility 
is hard. Finally, the scaffolds fabricated need to be characterized for long-term stability, degradation profiles, and long-term in vivo responses.

Author Contributions: Samerender Nagam Hanumantharao- writing-original draft preparation, conceptualization, data curation, methodology, investigation; Smitha Raoproject administration, writing-review and editing, supervision

Funding: This research received no external funding

Acknowledgments: The authors would like to acknowledge the support received by Samerender Nagam Hanumantharao through the Portage Health Foundation (PHF) graduate assistantship.

Conflicts of Interest: The authors declare no conflict of interest.

\begin{tabular}{|c|c|}
\hline $\mathrm{CA}$ & Cellulose Acetate \\
\hline $\mathrm{CF}$ & Chloroform; \\
\hline DCE & 1,2-dichloroethane \\
\hline DCM & Dichloromethane \\
\hline DMAC & Dimethylacetamide \\
\hline DMEM & Dulbecco Modified Eagle's Medium \\
\hline DMF & $\mathrm{N}, \mathrm{N}$-dimethylformamide \\
\hline DMSO & Dimethyl sulfoxide \\
\hline $\mathrm{ECM}$ & Extracellular Matrix \\
\hline GO & Graphene Oxide \\
\hline HA & Hydroxyapatite; \\
\hline HFP & 1,1,1,3,3,3-hexa-fluoro-2-propanol \\
\hline FESEM & Field Emission Scanning Electron Microscope \\
\hline HSA & Human Serum Albumin \\
\hline MWCNT & multiwalled-carbon nanotubes \\
\hline PANI & Polyaniline \\
\hline PBAT & Poly(butylene adipate-co-terephthalate) \\
\hline PBS & Phosphate buffered saline \\
\hline
\end{tabular}




\begin{tabular}{|c|c|}
\hline PCL & Poly-caprolactone \\
\hline PDMS & polydimethylsiloxane \\
\hline PEA & Poly(ester amide) \\
\hline PGA & Polyglycolide \\
\hline PGS & Poly(Glycerol Sebacate) \\
\hline PHB & Polyhydroxybutyrate \\
\hline PHBV & Poly(hydroxybutyrate-cohydroxyvalerate) \\
\hline PLA & Poly(lactic acid) \\
\hline PLGA & Poly(lactic-co-glycolic acid) \\
\hline PLLA & Poly(1-lactic acid) \\
\hline PLLA-CL & Poly(L-lactic acid-co-e-caprolactone) \\
\hline PMMA & Poly(methyl methacrylate) \\
\hline PPy & Polypyrrole \\
\hline PVA & Polyvinyl alcohol \\
\hline PVAc & Polyvinyl acetate \\
\hline PVDF & Polyvinylidene fluoride \\
\hline PVP & Polyvinyl pyrrolidone \\
\hline SF & Silk fibroin \\
\hline TFA & Trifluoracetic acid \\
\hline TFE & 2,2,2-Trifluoroethanol \\
\hline TFE & 2,2,2-Trifluoroethanol \\
\hline THF & Tetrahydrofuran \\
\hline
\end{tabular}




\title{
3 Self-Assembly of 3D Nanostructures in Electrospun Polycaprolactone-Polyaniline Fibers and their Application as Scaffolds for Tissue Engineering
}

\author{
Samerender Nagam Hanumantharao ${ }^{1}$, Carolynn Que $^{1},{ }^{*}$ Smitha Rao ${ }^{1}$ \\ ${ }^{1}$ Department of Biomedical Engineering, Michigan Technological University, Houghton, \\ MI 49931, USA
}

*Corresponding author: Email: smithar@mtu.edu

Received 15 December 2018, Accepted 16 March 2019, Available online 19 March 2019. https://doi.org/10.1016/j.mtla.2019.100296

Keywords: Electrospinning; Nanofibers; Scaffolds; 3D nanostructures

\begin{abstract}
:
The fabrication of synthetic scaffolds that mimic the microenvironment of cells is a crucial challenge in materials science. The honeycomb morphology is one such bio-mimicking structure that possesses unique physical properties and high packing efficiency in a 3dimensional space. Here, we present a novel method for electrospinning polycaprolactonepolyaniline with continuous, self-assembled, uniform, interwoven nanofibers forming patterns without the use of templates or porogens. By using the approach presented here, unique architectures mimicking the natural mechanical anisotropy of extracellular matrix were created by varying the electric field. Adult human dermal fibroblasts (HDFa) cells were successfully cultured on the nanofiber scaffolds without any external growth factors or post-processing of the nanofibers. Our data indicates that despite identical chemical composition, the physical properties impact cell attachment, alignment, and penetration into the scaffold. The mechanical strength of the fibers also plays a role with a distinct preference to fibers with high stiffness and ultimate tensile strength. Thus, by tuning the electric field, fibers with different physical properties and patterns can be fabricated for different applications.
\end{abstract}




\section{Graphical Abstract:}

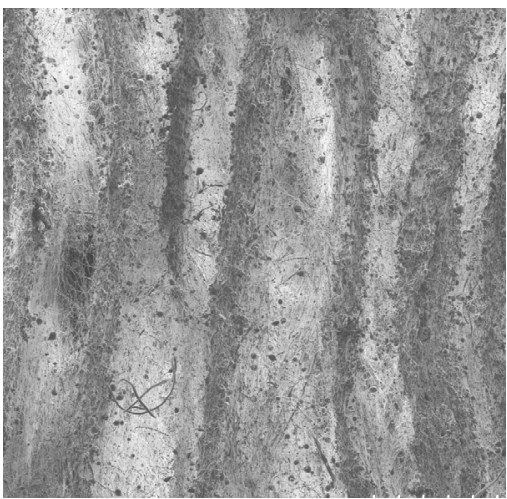

FESEM image of the honeycomb structured nanofibrous scaffold
Seeding scaffolds with Human Dermal Fibroblasts

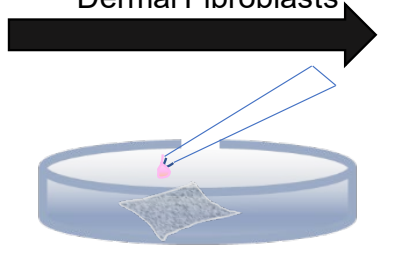

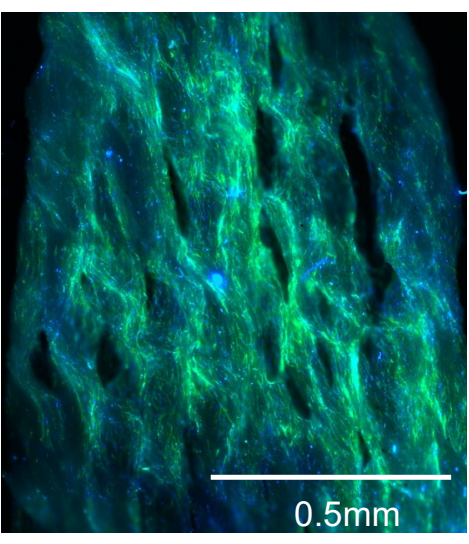

Scaffolds seeded with fibroblasts after Day 7. Blue: nucleus. Green: F-Actin

\subsection{Introduction}

The process of self-assembly refers to the efficient and spontaneous bottom-up arrangement of nanostructures with well-defined symmetry and long-range order [190]. Some of the applications of these self-assembled nanostructures are in designing catalysts [191], optics [192], batteries [193], supercapacitors [194], filters with molecular sieves [195], scaffolds for engineering the 3D microenvironment of tissues [196], and drug delivery applications [197]. Self-directed assembly of nanomaterials has been of considerable interest and has gained momentum recently due to advances in fabrication techniques and ability to automate the production. This is critical for the commercial production of functional structures using templating approaches with nanometer scale accuracy. The fabrication of the self-assembled nanostructures can be achieved by mixing colloidal solutions, nanostructured templating, self-organizing medium and wrapping [198]. However, as the complexity of the structure increases, the template and the selfassembled structures will involve integration of multiple processes each with their specific material and chemical requirements.

The formation of different nanostructures including nanodots and nanofibers, can be achieved through electrospinning, an electric-field assisted fabrication technique [199]. The advantages include low cost, quick processing speeds, long-term stability, ease of control and scalability. Nanofibers obtained from electrospinning have been reported for drug delivery [200], tissue scaffold engineering [201], energy storage devices [202], actuators [203], solid state devices [204], smart textiles [205], filters [206], optoelectronics [207], resonators [208], and wound healing [209]. However, the limitation is in efficiently producing nanostructures with high aspect ratio. To address this challenge, modifications such as low temperature electrospinning [210], self-assembly [211], replacement of 
collector with a template of required pattern or liquid bath [212-214], post-processing and additive manufacturing have all been reported [215].

The honeycomb topology can be broadly classified based on the shape of the 2D unit cell type such as square, circular, triangular and hexagonal which is stretched in columns to create $3 \mathrm{D}$ structures. The topology of the unit cell is optimized based on the required function. The hexagonal cells help in convective transfer whereas the triangular honeycomb possess superior mechanical properties. This makes the honeycomb structure extremely useful in designing complex nanostructures at the macro- and nanoscale. The packing and tiling of honeycomb structures is also very efficient which allows for high packing densities and interactions between multiple points in a plane [216]. Using electrospinning, the honeycomb structure has been previously reported for energy storage applications [217], tissue scaffold engineering [218], and for use as filters [219]. The pattern offers a wide range of flexibility in design by varying the pore size. Thandavamoorthy et al. demonstrated the production of self-assembled honeycomb structures in membranes using polyurethane [220]. Electrospraying and electrospinning was used by Wittmer to generate 3D honeycomb structures using a patterned collector [221]. Lavielle and colleagues used photolithography to make special patterned collectors composed of conducting and dielectric materials [222]. The fibers were collected in the conducting parts of the collectors and thus provided control over the 3D constructs. Reis and colleagues investigated the self-assembly of the nanofibers to form various $3 \mathrm{D}$ structures and the dependence on parameters in forming the structures [223]. The investigations were limited to homogenous polymers and the underlying nanofibers had no alignment. Since the pattern relied on the underlying template on the collector, altering the pores in the structures required changing the collector, thereby limiting dynamic alterations of the structures formed. Nedjari and colleagues fabricated honeycomb shaped scaffolds using a patterned template for tissue engineering studies [224]. While the study explored the effect of geometric cues provided by the honeycomb structures, the relation between the variations in the size of the pores to the cell behavior was not reported. Garcia et al. demonstrated the fabrication of honeycomb structures composed of PCL and hydroxyapatite using a patterned collector for bone tissue engineering [129]. A combination of electrospinning and electrospraying was used to obtain the combination of two polymers.

In this paper, a novel technique for self-assembly of nanofibers to form varying patterns of honeycomb structure using electrospinning is presented. This is the first study to report a template free method for the self-assembly of nanofibers to form a pattern with long-range order using polycaprolactone and polyaniline (PCL-PANI). A blend of polymers were used to obtain the required polarity to create the nanopatterns. The size of the pores formed was controlled by changing the electric field alone without any further changes to the electrospinning set-up. The 3D nanostructure thus obtained has the potential for use in drug delivery and energy storage applications with a demonstrated use in tissue engineering. 


\subsection{Experimental Section}

\subsubsection{Fabrication of Scaffolds}

All the materials were used as procured unless specified. Polycaprolactone (PCL): (MW = 70,000 GPC; Scientific Polymer Products, USA) and Polyaniline (PANI): Emeraldine base (MW = 50,000; Aldrich Chemistry, USA) were used for making the nanostructures. The doped form of PANI-PCL solution was prepared by dissolving equal concentrations of $0.35 \%$ wt. emeraldine base and 10-camphorsulfonic acid (CSA) (Aldrich Chemistry; France), and 15\% PCL in chloroform (Sigma Aldrich, USA) and filtering the solution using a syringe filter $(0.22 \mu \mathrm{m})$. The concentration of PANI in the filtered solution was determined post filtration using UV-vis spectrophotometer (LAMBDA 35; PerkinElmer, Inc). For electrospinning, the voltage was varied between $18-24 \mathrm{kV}$ in steps of $1 \mathrm{kV}$ to obtain different nanostructures using a rotating cylinder (EM-DIG and EM-RTC; IME Technologies, Netherlands) as collector. The environmental conditions were maintained as a constant. The tip-collector distance was maintained constant at $0.17 \mathrm{~m}$.

\subsubsection{Characterization of scaffolds}

The nanofibers were coated with a $5 \mathrm{~nm}$ thick coating of $\mathrm{Au} / \mathrm{Pd}$ before visualizing the fibers using a Field emission scanning electron microscope (FESEM; Hitachi S-4700 FE-SEM). The chemical bonds present in the scaffolds were analyzed using an Attenuated Fourier Transform Infrared Spectroscopy (Thermo Scientific ${ }^{\mathrm{TM}}$, Nicolet ${ }^{\mathrm{TM}}$ iS50 FTIR Spectrometer). Dynamic mechanical analyzer (TA Instruments ${ }^{\mathrm{TM}}$, DMA Q800) was used for characterizing the mechanical anisotropy of the scaffolds. The scaffolds were stretched along the direction of alignment of the fibers at isothermal conditions $\left(30^{\circ} \mathrm{C}\right)$. All the characterization experiments were repeated at least thrice to make sure the data was statistically significant.

\subsubsection{Cell Culture}

Adult Human Dermal Fibroblasts (HDFa) were purchased from America Type Cell Culture (ATCC ${ }^{\circledR}$ PCS-201-012 ${ }^{\mathrm{TM}}$ ), and were cultured in Dulbecco's modified eagle medium, DMEM, (Life Technologies, USA) supplemented with $10 \%$ fetal bovine serum (Life Technologies, USA) along with $1 \%$ Penicillin Streptomycin (Life Technologies, USA) under standard culture conditions $\left(37^{\circ} \mathrm{C}, 65 \% \mathrm{RH}\right.$ and $\left.5 \% \mathrm{CO}_{2}\right)$. The PCL-PANI scaffolds were cut into squares of $0.5 \mathrm{~cm}^{2}$ area (approximately), sterilized using ethanol, and irradiated in UV. Cells in exponential growing phase were trypsinized, counted using a Bright-Line $^{\mathrm{TM}}$ hemocytometer, seeded (2500 cells per scaffold) and cultured in the incubator under standard cell culture conditions. 


\subsubsection{Cell characterization}

Cells were visualized via fluorescence microscopy after 2, 3 and 7 days of growth. The cells were stained with DAPI (4',6-diamidino-2-phenylindole) (Life Technologies, USA) and Alexa Fluor ${ }^{\circledR} 488$ Phalloidin (Life Technologies, USA) for nuclei and cytoskeletal FActin visualization, respectively according to manufacturer's protocols.

\subsubsection{Cell Viability}

The proliferation studies of fibroblasts on the scaffolds were determined using the CellTiter-Blue ${ }^{\circledR}$ Cell Viability Assay (Promega, Madison, WI). A commercially available wound healing dressing (Hollister Endoform Dermal Template Collagen Dressing) was used as the positive control. A $10 \%$ DMSO solution was added to blank scaffold to serve as the negative control. The scaffolds including the positive control were cut into squares of side $0.5 \mathrm{~cm}(\mathrm{n}=3)$. At Days 1, 3 and 5 culture media was replaced and $20 \%$ volume of CellTiter-Blue ${ }^{\circledR}$ Cell Viability Assay reagent was added and incubated for $4 \mathrm{~h}$ under standard culture conditions $\left(37^{\circ} \mathrm{C}, 65 \% \mathrm{RH}\right.$ and $\left.5 \% \mathrm{CO}_{2}\right)$.. A $75 \mu \mathrm{L}$ aliquot of the incubated medium was transferred to a 96-well opaque culture plate (Corning Glass Works), and determined using a plate reader (Beckman Coulter DTX 880 Multimode Detector) at 560 $\mathrm{nm}$ excitation and $590 \mathrm{~nm}$ emission. The standard error bars were calculated by determining the standard error of mean.

\subsection{Results and Discussion}

The self-assembly of the honeycomb structures is a multi-step process which begins with the preparation of the polymer blends. The polymer-solvent interactions play a significant role in not only influencing the viscoelasticity and polarity of the solution but also in the morphology, topology, crystallinity, electroactivity and mechanical properties of the fibers. Polycaprolactone (PCL), a commonly used viscoelastic polymer was chosen because of its high elasticity, biocompatibility, biodegradability and its solubility in a wide range of solvents. Polyaniline (PANI) is a semiconducting polymer that is known to be conductive in its salt form. The emeraldine salt is obtained by addition of CSA as a dopant. A miscible binary polymer blend was prepared using chloroform. Chloroform was selected as the solvent because of its high intrinsic viscosity, low boiling point and electrical conductivity among all the other organic solvents. Concentrations of PANI and PCL in the blend was prepared according to their solubility parameters in chloroform $[225,226]$. The concentration of PANI in the PCL-PANI blend post-filtration was characterized to be $0.4 \mathrm{mg} / \mathrm{mL}$ using UV-visible spectrophotometry (Figure S1). The concentration of PANI dispersed is low because of the solvent used and the presence of a secondary polymer (PCL) in the blend. The PANI is present in its salt form in the PCL-PANI blend which can be interpreted by the presence of peaks corresponding to polaron and exciton transitions [227]. The range at which a polymer blend can be electrospun is very narrow [228]. One of the important determinants of electrospinning is the formation of a Taylor cone which is dependent on the surface tension of the polymer blend and applied electric field among 
others. A stable Taylor cone-jet is essential for continuous electrospinning and the change in the Taylor cone formed affects the morphology of the nanofibers [229]. In our study reported here, except for the voltage applied to the nozzle, all the parameters used for the electrospinning process to obtain the different honeycomb patterns were kept constant.
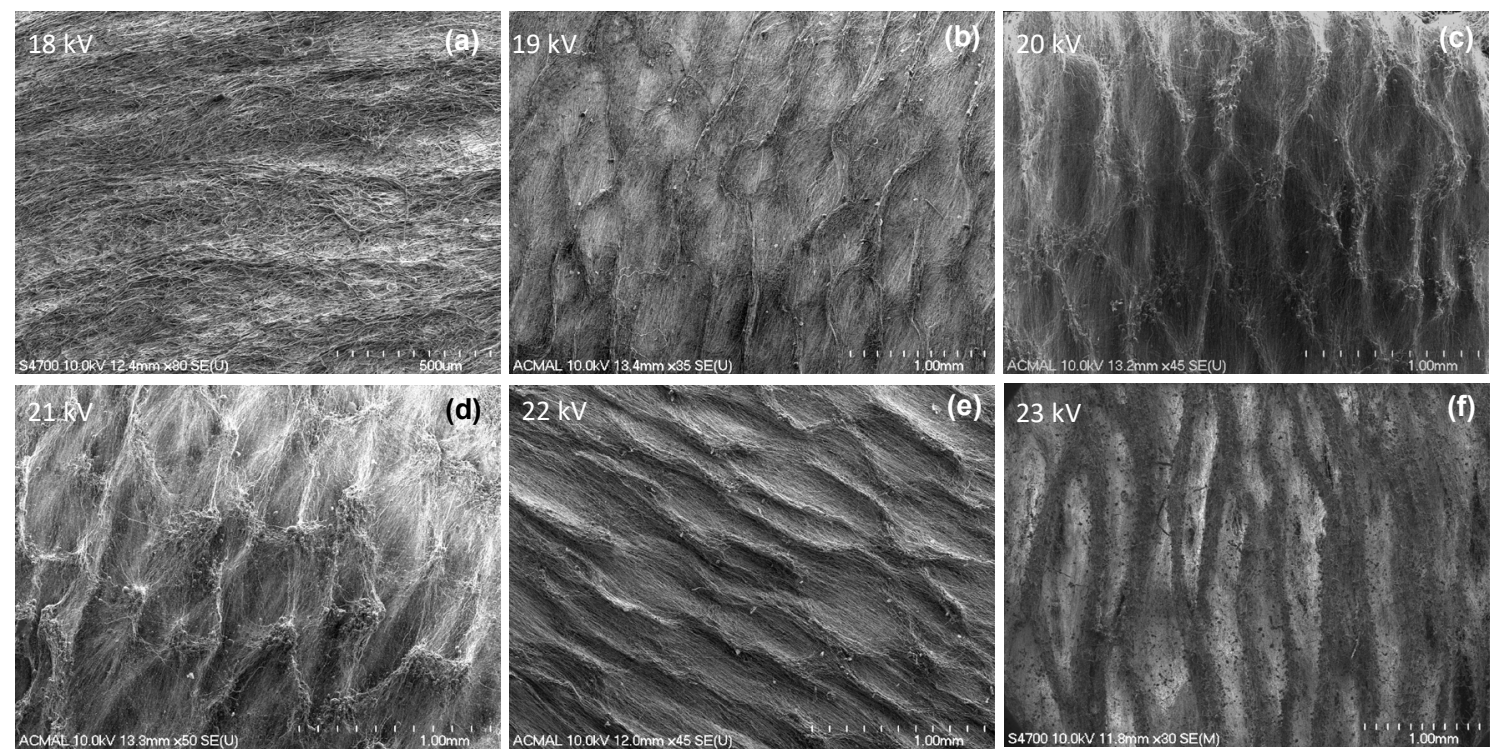

Figure 1. Field emission scanning electron microscopic (FESEM) images of the PCLPANI scaffolds exhibiting porous morphology and fiber alignment. PCL-PANI-18kV had a wavy architecture without pores (a). The PCL-PANI-19kV the pore formation is visible but is more pronounced in PCL-PANI 20kV and PCL-PANI-21kV (b, c, d). In PCL-PANI$22 \mathrm{kV}$, the structures were elongated and incomplete (e). In PCL-PANI-23kV elongated, complete and repeated structures are formed (f).

The low and high magnification FESEM images provide information to relate the effect of electric field during electrospinning to the morphology of the fibers. Low magnification FESEM images of the honeycomb structures formed by electrospinning at different voltages is shown in figure 1(a) through 1(f) and higher magnification FESEM images is shown in supplementary figures S2-S7. At $18 \mathrm{kV}$, the fibers obtained have little to no alignment and there are no interwoven structures or beading, as can be seen from figure 1(a). The higher magnification image (figure S2) shows the formation of clusters of fibers in regions which indicate that a higher electric field (voltage or change in tip-collector distance) and time was needed for the fibers to be elongated. The PCL-PANI-19kV, in figure 1(b) have interconnected pores which are small and uneven in arrangement. The honeycomb arrangement cannot be seen uniformly across the entire surface. A higher magnification image (figure S3) of the fibers revealed aligned fibers that are concentrated towards the beads. The underlying structure of the fibers were well aligned and fibrous with little or no beads. This confirms that the formation of beads is not an initial process but occurs at a later point of time during the electrospinning. In PCL-PANI-20kV (figure $1 \mathrm{c})$, the pores are uniaxially aligned and larger than the pores from $19 \mathrm{kV}$, with the presence of beading along the perimeters of the pores but not in the other regions. However, a higher 
magnification (figure S4) of the scaffold's underlying structure showed beading and a wavy alignment with overlapping layers of fibers forming a tightly packed structure. In PCL-PANI-21kV (figure 1d) deeper pores compared to PCL-PANI-20kV can be observed. A high magnification (figure S5) of the structure showed the presence of beads and the alignment of the underlying architecture. The fibers are attracted more towards the area containing beads and thus create pores. The honeycomb pattern in the PCL-PANI-22kV (figure 1e) is stretched out and incomplete and the underlying fibers are aligned with little or no beading. The higher magnification image (figure S6) showed the underlying structure is completely devoid of beads and the droplets. This change in size of pores and increase in beading is more evident as the voltage is increased. The PCL-PANI-23kV (figure 1f) sample is continuous and has long-range order throughout in a symmetrical manner. The underlying alignment is limited to a few layers of the fibers. A high magnification (figure S7) of the regions between the walls of the honeycomb structure and the base layer showed a large concentration of droplets on the walls of the honeycomb structure. The beads act as points of attraction for the fibers to form and this creates a dense network. On a macroscale (millimeter scale) this leads to the formation of a pattern, a honeycomb structure in this case. Varying the electric field intensity altered the formation, the size of pores, and the nature of the fiber between non-uniform, wavy and straight, since all the other parameters were constant. A high contrast FESEM image (figure S9) of the fibers helps in highlighting the regions of fiber formation and the size of the pores.

The fibers were collected at a high uptake velocity which decreases the distance between two individual large diameter fibers and at the same time increases alignment rate of the smaller diameter fibers [230]. The underlying structure in each case is a layer of fibers or beads which have a directionality along the rotation of the collector. The time point at which the beads start collecting on the surface of the fibers over the duration of electrospinning is dependent on the electric field. This process fails to start in the case of the fibers electrospun at $18 \mathrm{kV}$ and happens almost immediately in the fibers electrospun at $24 \mathrm{kV}$ (figure S7). The formation of the beads on the fibers causes a change in the distribution of the electrostatic charges. The beads behave in a manner similar to point charges, attracting the fibers towards them. A similar mechanism was used by Sun et al. to fabricate 3D spongiforms [231]. The beads formed have repeated patterns because of the Rayleigh instability and splaying on a rotating collector, finally forming the interconnected honeycomb morphology. The change in the dimensions of the honeycomb structure is in accordance with finding by Reis et al.[223]. Briefly, the experimental verification for the in situ polarization and the factors responsible for electrodynamic tailoring was studied. It was found that the initial regions of high fiber density act as the motifs for the selfassembled process. Attraction to the motifs and repulsion from the other areas favors the growth of the nanostructures. The polarity of the fibers leads to the formation of wavy fibers. The wavy fibers are absent at an increased electric field leading to formation of straight fibers. Increasing the electrical field also increases the surface charge density during electrospinning affecting the shape of the Taylor cone [232]. The formation of the Taylor cone was discontinuous at electric field intensities lower than $1.05 \mathrm{kV} / \mathrm{cm}$. This was due to surface tension and viscosity of the droplet at the needle. The size of the pores of the honeycomb increases gradually with increase in electric field. The Taylor cone then 
recedes into the needle when the applied voyage is $22 \mathrm{kV}$. When the voltage applied is increased to $24 \mathrm{kV}$, highly beaded structures with very few fibers were formed.
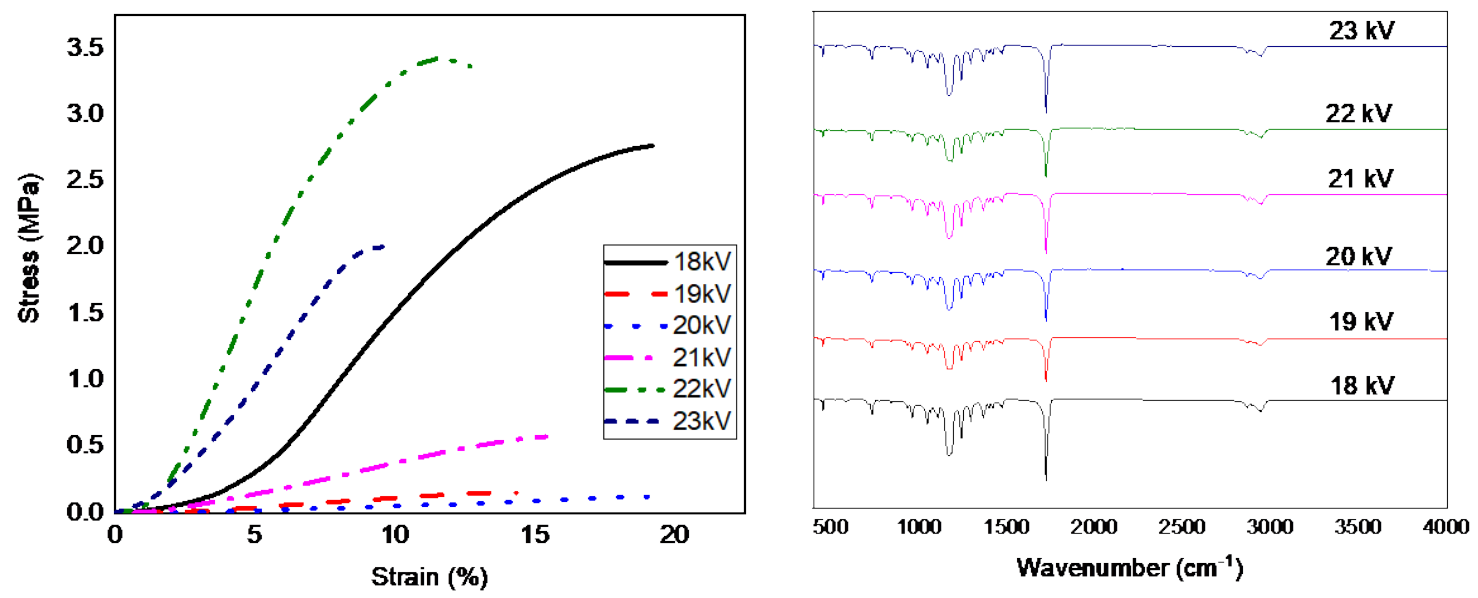

Figure 2A. Mechanical analysis of the different scaffolds illustrated by the stress-strain profiles. PCL-PANI-22kV had the highest ultimate tensile strength while PCL-PANI-20kV had the lowest tensile strength. Figure 2B. Surface analysis of the different scaffolds illustrated by the ATR-FTIR spectra. All the scaffolds had the same functional groups and there was no considerable change in area of the peaks of interest indicating similar surface chemistries between all the scaffolds.

The surface chemistry of the nanofibrous scaffolds were analyzed using ATR-FTIR. A $\%$ Transmittance versus wavenumber plot was generated and signature peaks were identified as shown in data from figure $2 \mathrm{~A}$. The characteristic peaks of PCL, such as the $\mathrm{C}=\mathrm{C}, \mathrm{C}=\mathrm{O}$ are present in all the spectra. The strong intensity peaks at $1722 \mathrm{~cm}^{-1}(\mathrm{C}=\mathrm{O}$ stretching) and 1167 (C-O stretching), and medium intensity peaks at $2944 \mathrm{~cm}^{-1}$ (asymmetric $\mathrm{CH}_{2}$ stretching) and $2866 \mathrm{~cm}^{-1}$ (symmetric $\mathrm{CH}_{2}$ stretching) are unique to PCL. The strong intensity peak at $1293 \mathrm{~cm}^{-1}$ is specific to the $\mathrm{C}-\mathrm{O}$ and $\mathrm{C}-\mathrm{C}$ stretching in the crystalline phase of PCL. The weak intensity peak at $582 \mathrm{~cm}^{-1}$ is specific to the $\mathrm{C}=\mathrm{O}$ wag in PCL. The characteristic peaks of PCL at $1231 \mathrm{~cm}^{-1}$ (asymmetric C-O-C stretching) and $1176 \mathrm{~cm}^{-1}$ (C-O stretching) confirm the presence of PCL in the PCL-PANI sample. PANI has characteristic peaks in the 3000-3500 region, which is specific to the amine group. The $\mathrm{N}-\mathrm{H}$ stretching at $3442 \mathrm{~cm}^{-1}$ is specific to PANI. The percentage transmittance of the peaks remained almost similar without significant changes across the scaffolds fabricated at different voltages as inferred from figure S10 and S11.

The mechanical properties of the scaffolds were inferred from the stress-strain plot in figure $2 \mathrm{~B}$ and the analysis from the plot is tabulated in table 1 . The mechanical properties of the scaffold are an important characteristic for cell proliferation, differentiation and migration. The variability in mechanical properties of the scaffolds can also be related with their structures as seen using FESEM. Youngs modulus of the fibers, Ultimate tensile strength and elongation at failure for the scaffolds were extrapolated from the stress-strain relationship of the scaffold. The scaffolds which had a higher percentage of beads have 
poor mechanical strength indicated by their ultimate tensile strength. The PCL-PANI$19 \mathrm{kV}, 20 \mathrm{kV}$ and $21 \mathrm{kV}$ exhibited poor tensile strength and breakage at low strains. The PCL-PANI-18kV, 22kV and $23 \mathrm{kV}$ exhibited high stiffness and ultimate tensile strength, which is ideal for culturing fibroblasts $[233,234]$. Thus, the voltage used for fabricating the honeycomb structures enabled modification of the mechanical properties of the scaffold based on the required application [235].

Table 1. Mechanical Analysis of the different samples calculated from the stress-strain profiles.

\begin{tabular}{|c|c|c|c|c|c|c|}
\hline Sample & $\begin{array}{l}\text { PCL- } \\
\text { PANI- } \\
18 \mathrm{kV}\end{array}$ & $\begin{array}{l}\text { PCL- } \\
\text { PANI- } \\
19 k V\end{array}$ & $\begin{array}{l}\text { PCL- } \\
\text { PANI- } \\
20 \mathrm{kV}\end{array}$ & $\begin{array}{l}\text { PCL- } \\
\text { PANI- } \\
21 \mathrm{kV}\end{array}$ & $\begin{array}{l}\text { PCL- } \\
\text { PANI- } \\
22 \mathrm{kV}\end{array}$ & $\begin{array}{l}\text { PCL- } \\
\text { PANI- } \\
23 \mathrm{kV}\end{array}$ \\
\hline $\begin{array}{l}\text { Youngs } \\
\text { Modulus } \\
(\mathrm{MPa})\end{array}$ & $\begin{array}{l}0.178 \pm 0.0 \\
8\end{array}$ & $\begin{array}{l}0.009 \pm 0.0 \\
03\end{array}$ & $\begin{array}{l}0.009 \pm 0.0 \\
04\end{array}$ & $\begin{array}{l}0.051 \pm 0.0 \\
1\end{array}$ & $\begin{array}{l}0.42 \pm \\
0.05\end{array}$ & $\begin{array}{l}0.271 \pm 0.0 \\
1\end{array}$ \\
\hline $\begin{array}{l}\text { Ultimate } \\
\text { Tensile } \\
\text { Strength } \\
(\mathrm{MPa})\end{array}$ & $\begin{array}{l}2.089 \pm 0.7 \\
1\end{array}$ & $\begin{array}{l}0.179 \pm 0.0 \\
4\end{array}$ & $\begin{array}{l}0.148 \pm 0.0 \\
2\end{array}$ & $\begin{array}{l}0.506 \pm 0.0 \\
7\end{array}$ & $\begin{array}{l}3.68 \pm \\
0.18\end{array}$ & $\begin{array}{l}1.748 \pm 0.2 \\
2\end{array}$ \\
\hline $\begin{array}{l}\text { Elongatio } \\
\mathrm{n} \text { at } \\
\text { Fracture } \\
(\%)\end{array}$ & $\begin{array}{l}20.848 \pm 3 \\
98\end{array}$ & $\begin{array}{l}33.873 \pm \\
17.68\end{array}$ & $\begin{array}{l}38.337 \pm 2 \\
0.02\end{array}$ & $\begin{array}{l}23.030 \pm 1 \\
4.90\end{array}$ & $\begin{array}{l}13.47 \pm \\
0.73\end{array}$ & $\begin{array}{l}9.112 \pm 0.8 \\
4\end{array}$ \\
\hline
\end{tabular}
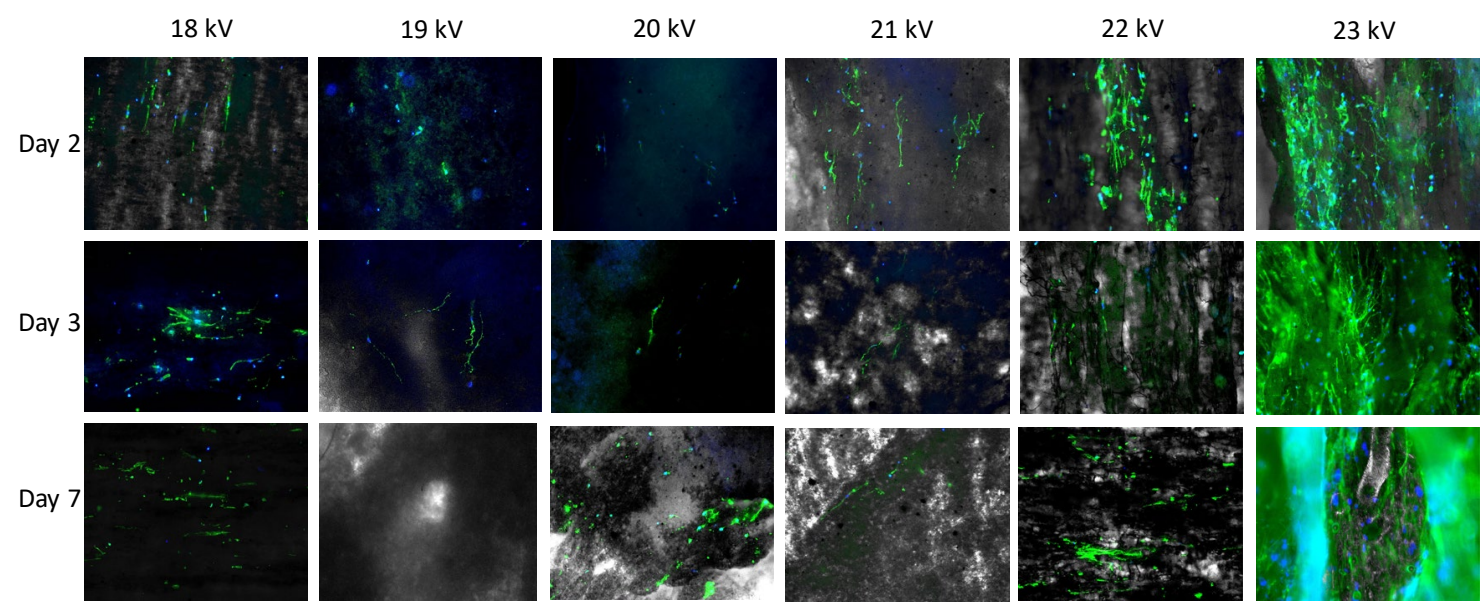
Figure 3. Fluorescent and brightfield overlay microscope images of adult dermal human fibroblast (HDFa) cells on the different scaffolds on days 2,3 and 7. There appears to be a greater degree cell attachment and alignment on PCL-PANI-23kV for all days. The nuclei were stained with DAPI (blue) and F-actin filaments were stained with Alexa Fluor ${ }^{\circledR}$ Phalloidin (green). Images are at 10X magnification.

In order to demonstrate feasibility of using PCL-PANI scaffolds in wound healing applications, we carried out cell studies using Adult Human dermal Fibroblast cells (HDFa). The cells act as a good model for in vitro wound healing studies [236]. The scaffolds after cell seeding were fixed and stained with DAPI and Alexa Fluor ${ }^{\circledR} 488$ Phalloidin to visualize the nucleus and the cytoskeleton (F-actin), respectively as shown in figure 3. The cells were seeded on the sterilized scaffolds without further post-processing or addition of factors to enhance adhesion. Based on the localization of the cells observed after 2 days, they appear to accumulate more towards the edges of the honeycomb structure. The number of cells is more in PCL-PANI-23kV compared to the other scaffolds. From the images, on day 3 , the cells aligned along the direction of alignment of the fibers. By day 7 , the cells proliferated along the depth of the scaffold and spread uniformly across the entire scaffold. The cells in PCL-PANI-23kV had the highest cell infiltration while PCLPANI-19kV had the lowest cell number. The direction of cell alignment was inferred from the brightfield overlay on the fluorescent images outlining the F-actin filaments. The outof-focus regions in the images are from the multiple layers of the electrospun fiber forming the scaffold. The stiffness of the scaffold helps the cells adhere and grow on it.
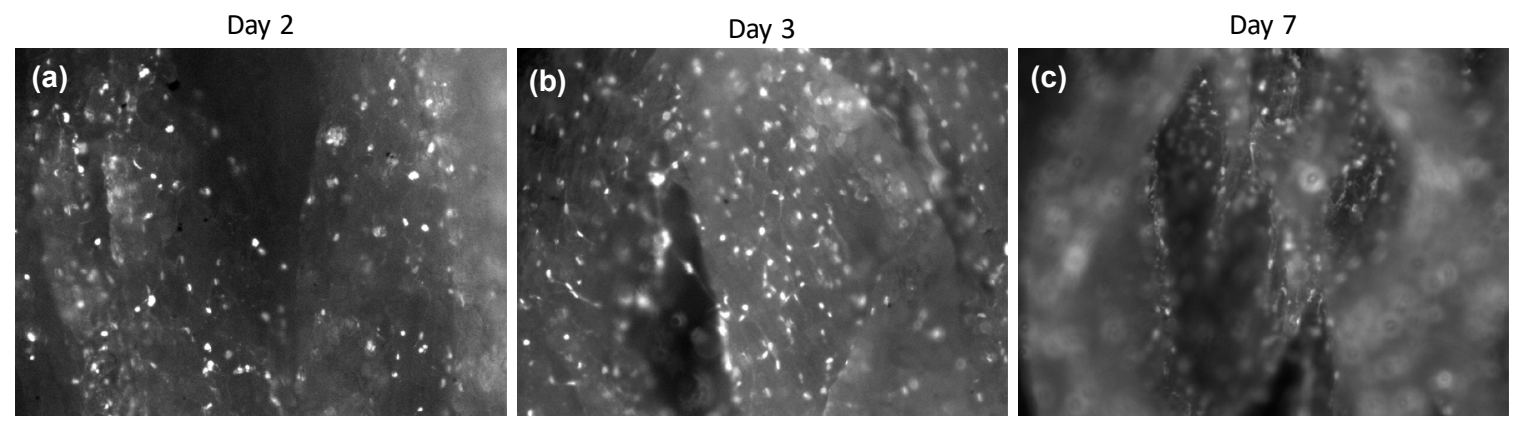

Figure 4. Grayscale images of the fluorescent signals obtained from cell nuclei stained with DAPI (white) demonstrating the localization and infiltration of the dermal fibroblasts on the PCL-PANI-23kV honeycomb structures. The cells remained on the surface on day 2 (a), show proliferation on the surface with some cell infiltration on day 3 (b) with complete infiltration on day 7 (c). All the images were taken at a magnification of $10 \mathrm{X}$.

The shape of the nucleus at different points of the cell seeding for PCL-PANI-23kV (figure 4) shows the synergy of cell, scaffold and typology of the scaffold. The structural characteristics of the scaffold enabled penetration of cells into the lower layers of the scaffold. The cells on day 2 were spread on the upper region of the scaffold. The cell number increased significantly as seen from the number of cells on day 3 . There was some level of cellular infiltration on day 3. The cells from day 7 had completely infiltrated 
towards the bottom layer of the scaffold. This sort of cell-scaffold interaction helps in increased cell numbers during tissue engineering studies which is essential in applications such as wound healing patches and organ engineering. The PCL in the fibers helps in increasing the elasticity and increase the rupture point of the scaffold.

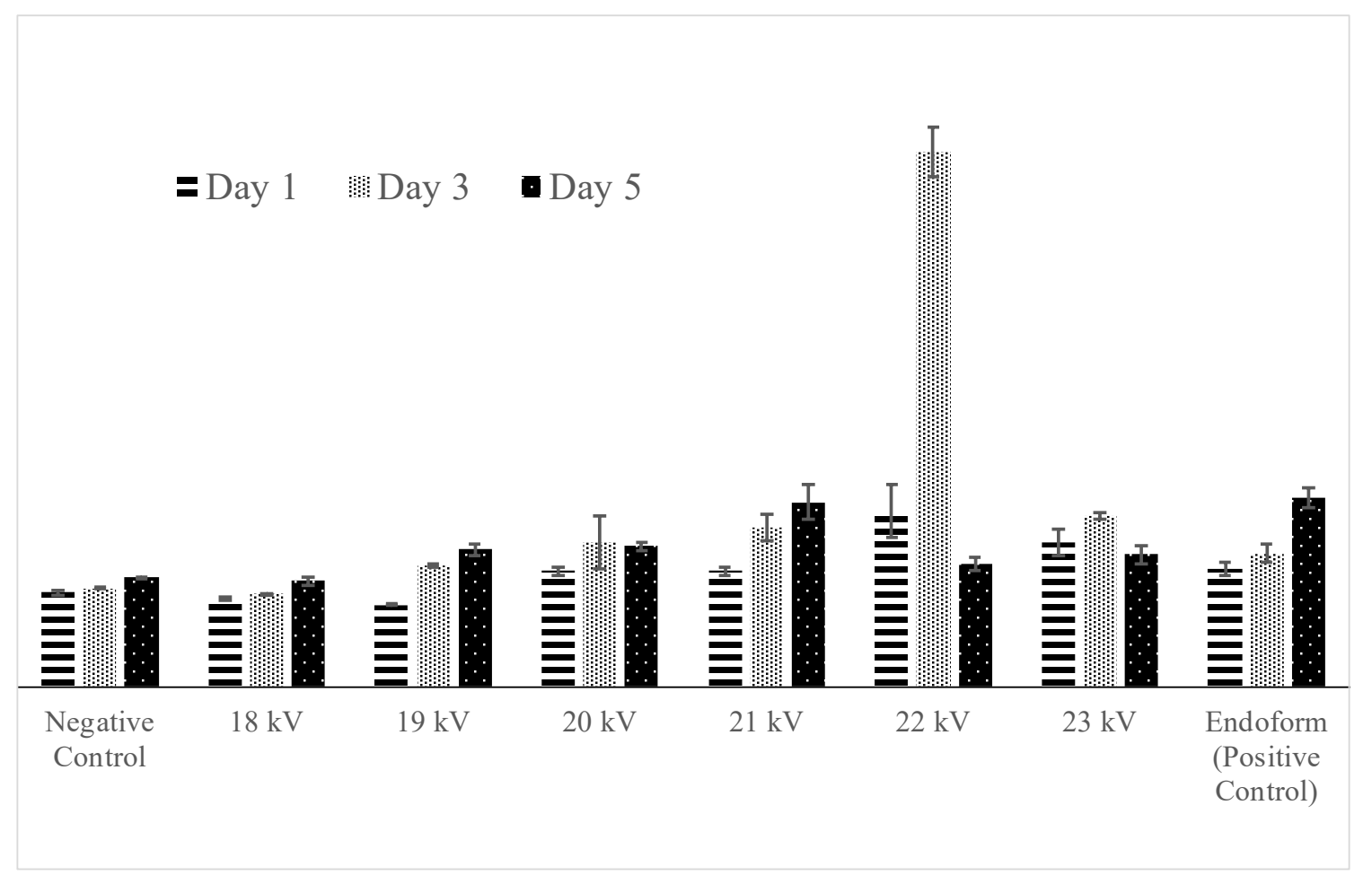

Figure 5. Quantification of cell viability and proliferation on all the scaffolds and controls on Days 1, 3 and 5. Among all the scaffolds, PCL-PANI-22kV and PCL-PANI-23kV had the highest cell viability.

The CellTiter-Blue cell viability assay (Figure 5) revealed that the fibroblasts proliferated differently on each scaffold. The cells adhered better and proliferated more freely on PCLPANI 20kV, PCL- PCL-PANI-21kV, PCL-PANI-22kV and PCL-PANI-23kV. The initial cell proliferation was highest on PCL-PANI-22kV followed by PCL-PANI-23kV. On day 3 , the rate of cell proliferation doubled than the rate on day 1 on PCL-PANI-22kV. However, on day 5, the cell proliferation decreased for PCL-PANI-22kV and PCL-PANI$23 \mathrm{kV}$. This can be attributed to the limited nutrient supply available to the cells since there was no change of media during the study. The cell proliferation was limited on all the three days on PCL-PANI-18kV and PCL-PANI-19kV and was comparable to the negative control. The cell proliferation behavior on PCL-PANI-21kV was similar to the positive control. The PCL-PANI-23kV and PCL-PANI-22kV have favorable properties for designing an ideal scaffold for wound healing applications. However, PCL-PANI-23kV provides a stable microenvironment for the cells to infiltrate and migrate along the scaffold. The cell proliferation data agrees well with the cell seeding images obtained after immunofluorescent staining. The morphology of the scaffold plays a major role in the 
alignment, infiltration and proliferation of the cells. The cells on PCL-PANI $23 \mathrm{kV}$ have elliptical nucleus and elongated morphologies with the cells distributed throughout the scaffold. The stiffness of the scaffold makes it mechanically compliant for the cells to adhere and proliferate. Furthermore, since the topology and mechanical characteristics are unique to each tissue, the current method of self-assembly could potentially be used as scaffolds for other tissues based on their unique properties.

\subsection{Conclusions}

Here we have successfully demonstrated self-alignment and alteration of the pore size and alignment of electrospun PCL-PANI honeycomb structures without the use of a template. The electric field was controlled to alter the distribution, size and structure of the pattern on the fiber scaffolds. The fibers were characterized for their mechanical strength and surface chemistry, and cellular compatibility was demonstrated using adult human fibroblast cells (HDFa). PCL-PANI-23kV had the best cellular growth and infiltration among all the other scaffolds.

\section{Acknowledgements}

The authors acknowledge the financial support provided by the Department of Biomedical Engineering at Michigan Technological University. The work was partially supported by financial contribution from Ken Gunther, an alumnus of Michigan Tech. The authors would also like to acknowledge Owen Mills at Applied Chemical and Morphological Analysis Laboratory (ACMAL) at Michigan Technological University and Dr. Kathryn Perrine, Department of Chemistry for their help with characterization and Infrared Spectroscopy.

\section{Declaration of Interest}

None

\section{Data Availability}

The processed data required to reproduce these findings have been shared (doi:10.17632/978kbhm3yw.1). The raw data obtained cannot be shared at this time as the data also forms part of an ongoing study.

\subsection{Supplementary Information}



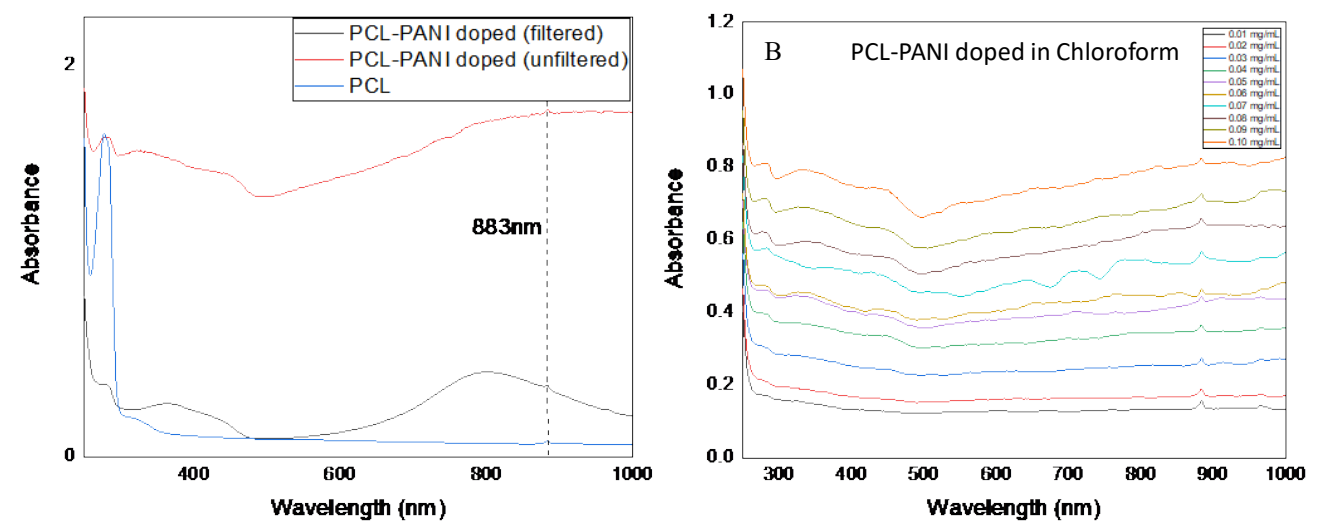

Figure S1. UV-visible spectrum of PCL-PANI solutions to calculate the concentration of doped PANI in the polymer blend post-filtration. The UV-visible spectra of PCL dissolved in chloroform, PCL-PANI doped pre- and post- filtration is represented in figure S1 A. Broad peaks at 349-362 nm corresponds to the transition of electrons in the benzenoid ring while the peaks around $433-444 \mathrm{~nm}$ and $782-815 \mathrm{~nm}$ corresponds to the polaron and exciton transition respectively [39]. The calibration curve for the doped PANI was obtained from the spectra represented in figure S1 B.
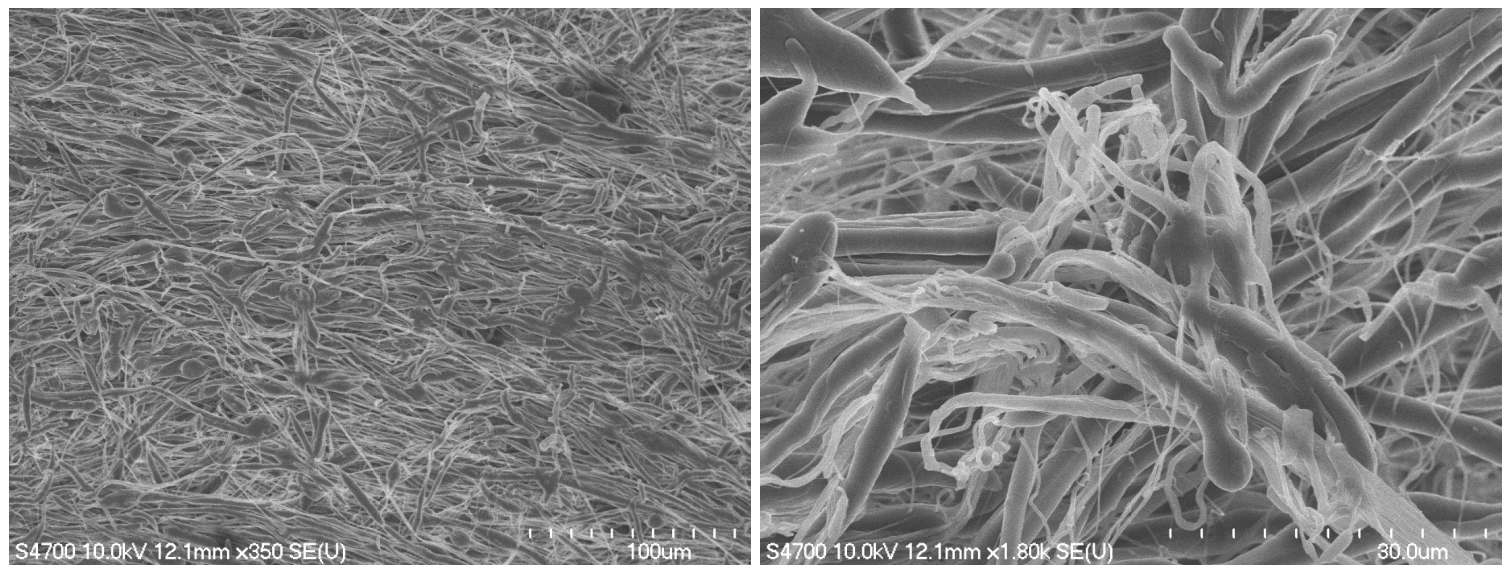

Figure S2. High magnification Field emission scanning electron microscopic (FESEM) images of the PCL-PANI-18kV scaffolds. The scaffolds have a wavy architecture. The fibers form clusters in regions which indicate that a higher electric field or uptake velocity was needed to improve alignment during the fabrication process. 

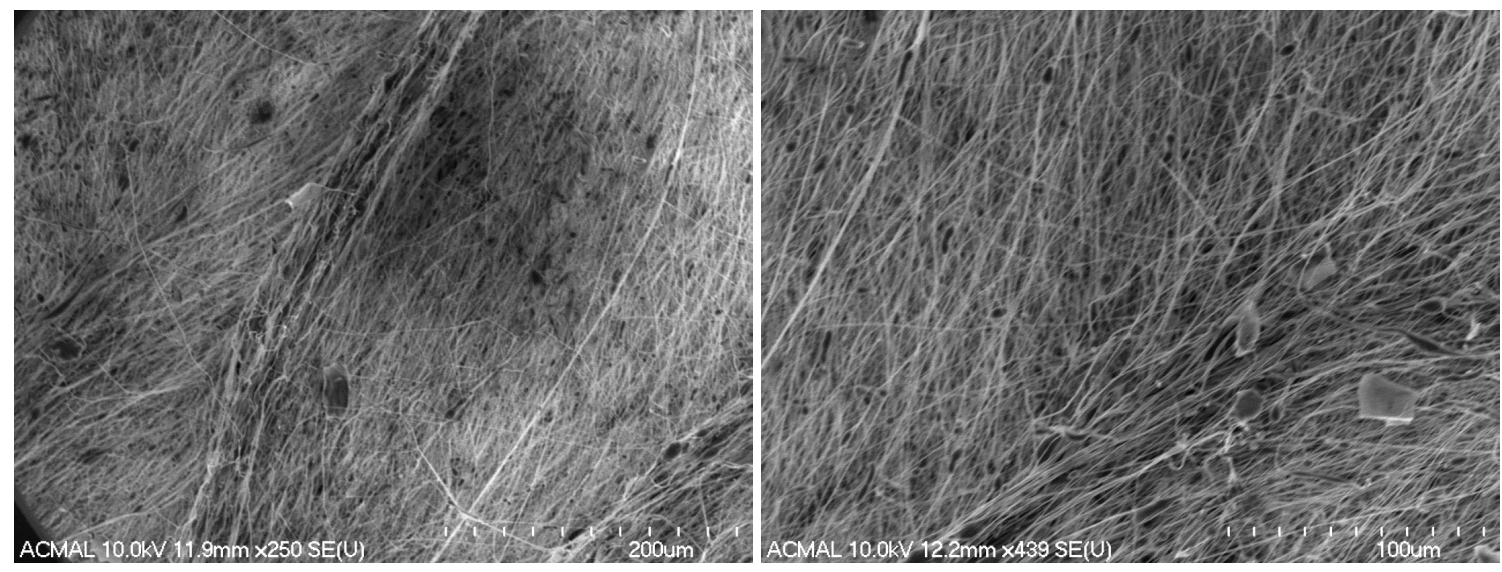

Figure S3. High magnification Field emission scanning electron microscopic (FESEM) images of the PCL-PANI-19kV scaffolds. The scaffolds had better alignment compared to PCL-PANI-19kV. The fibers accumulate around the droplets formed leading to the formation of pores.
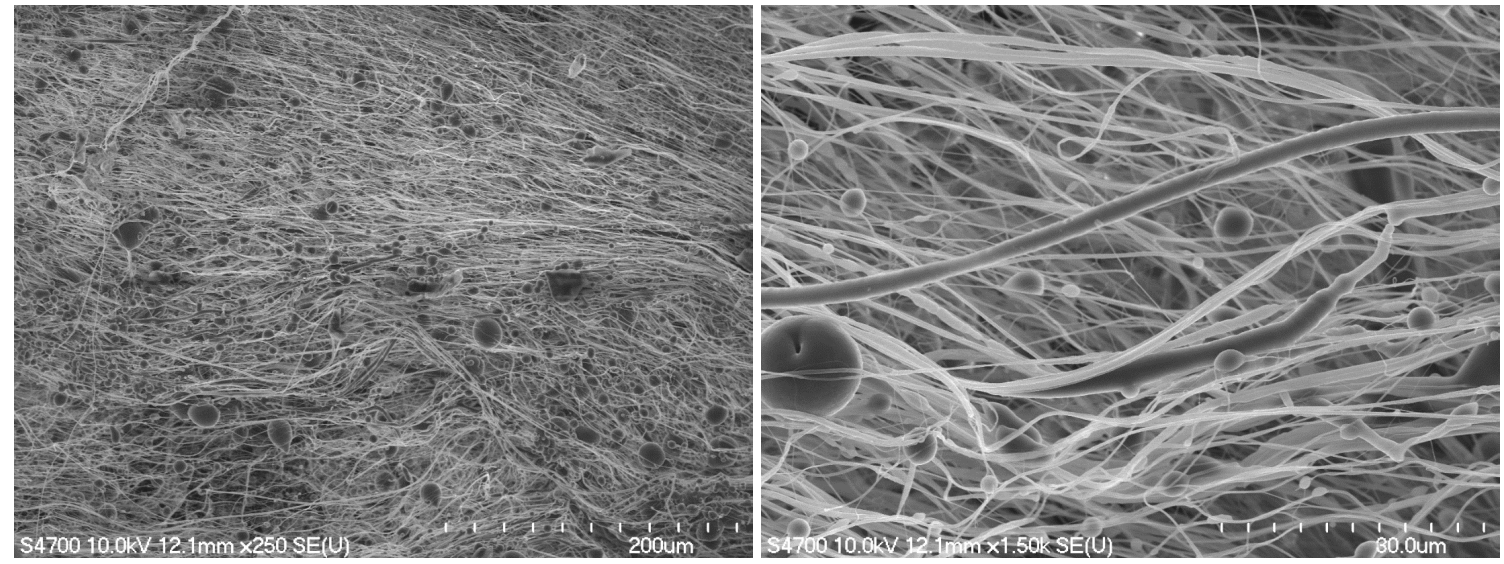

Figure S4. High magnification Field emission scanning electron microscopic (FESEM) images of the PCL-PANI-20kV scaffolds. The number of droplets increased with the increase in voltage. The underlying layer had fibers with a wavy architecture. 

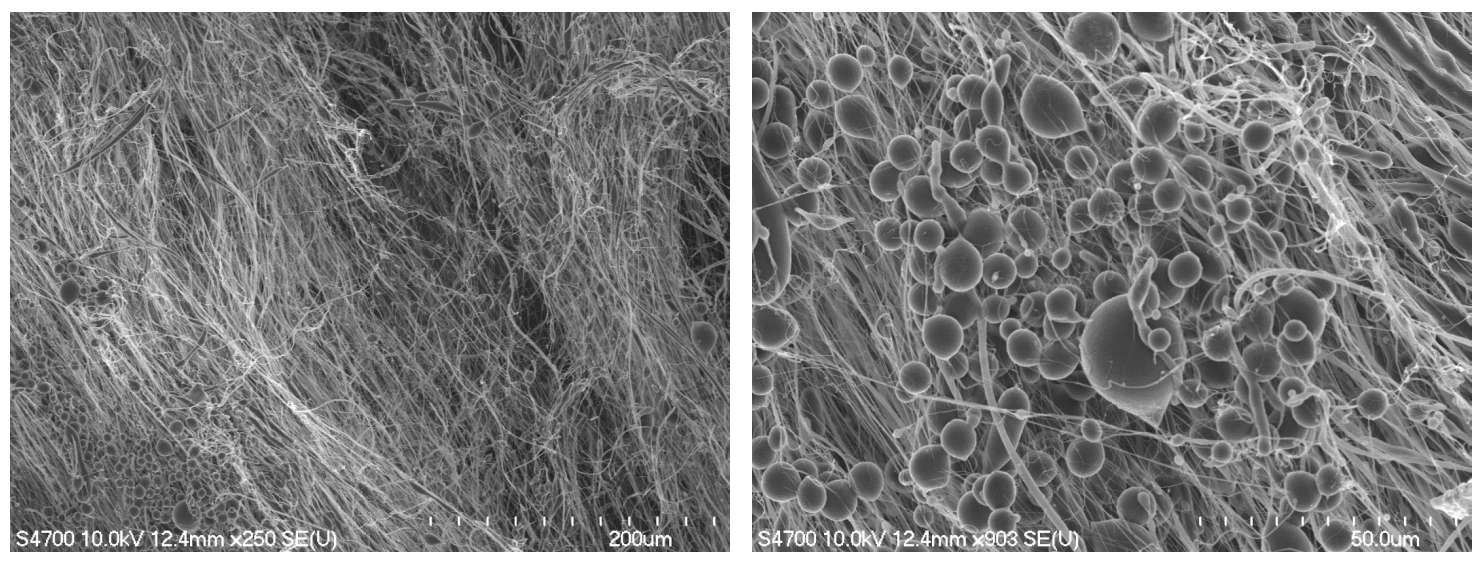

Figure S5. High magnification Field emission scanning electron microscopic (FESEM) images of the PCL-PANI-21kV scaffolds. A large concentration of droplets leads to pronounced and deep pores originating around the droplets.
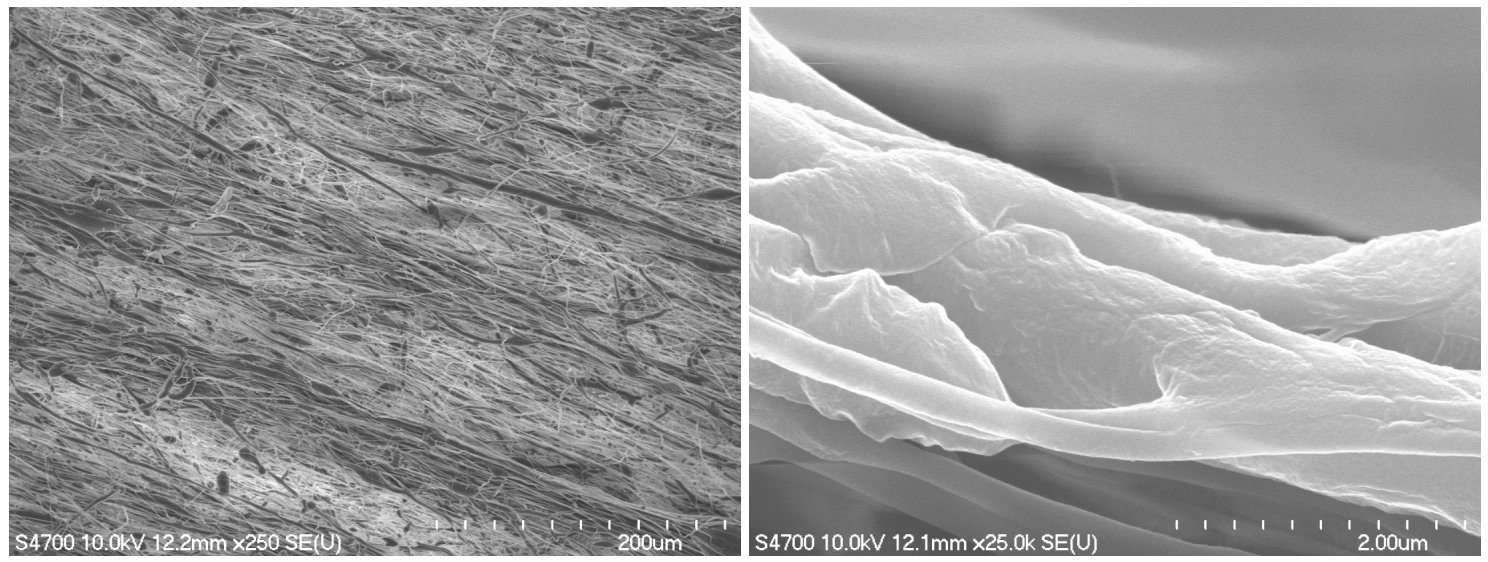

Figure S6. High magnification Field emission scanning electron microscopic (FESEM) images of the PCL-PANI-22kV scaffolds. The fibers were uniformly aligned with close packing leading to overlapping between the fibers. The smaller diameter nanofibers were formed from splitting of the larger diameter fibers leading to a rough surface appearance. 

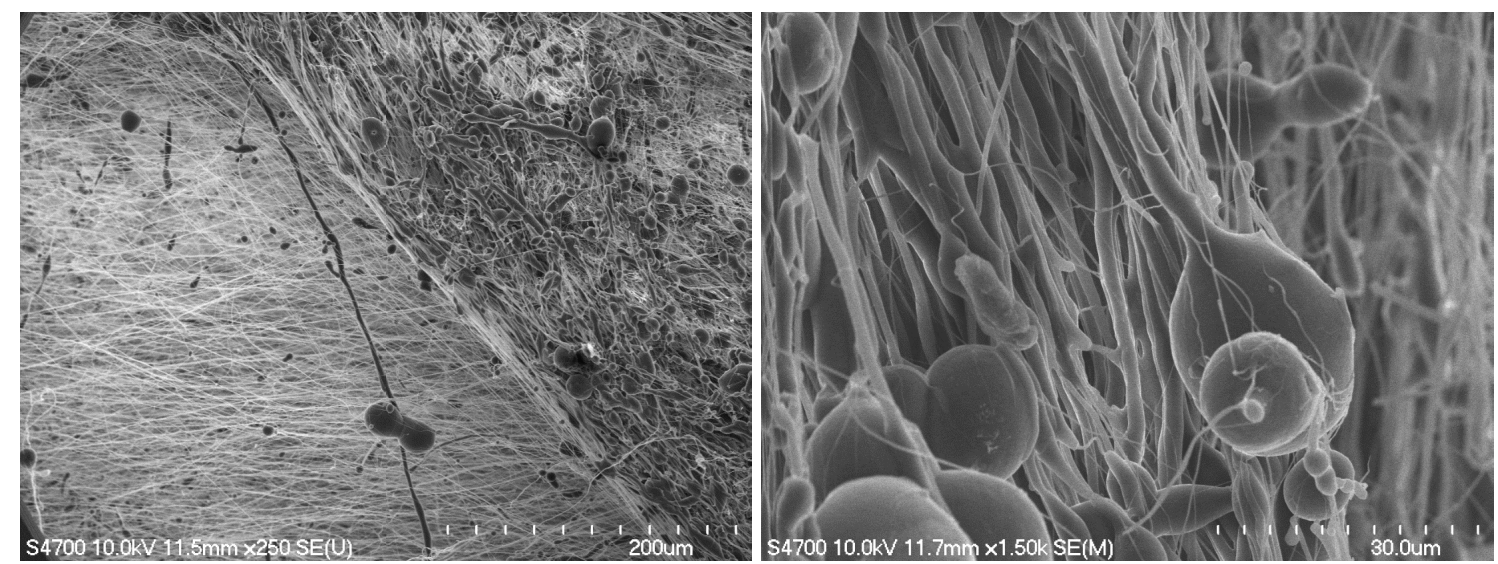

Figure S7. High magnification Field emission scanning electron microscopic (FESEM) images of the PCL-PANI-23kV scaffolds. There were two distinct regions that appear as the top and the bottom layer and form the elongated repeated patterns. The walls forming the structure were lined with a very dense concentration of droplets and fibers leading to the unique physical appearance and characteristics.
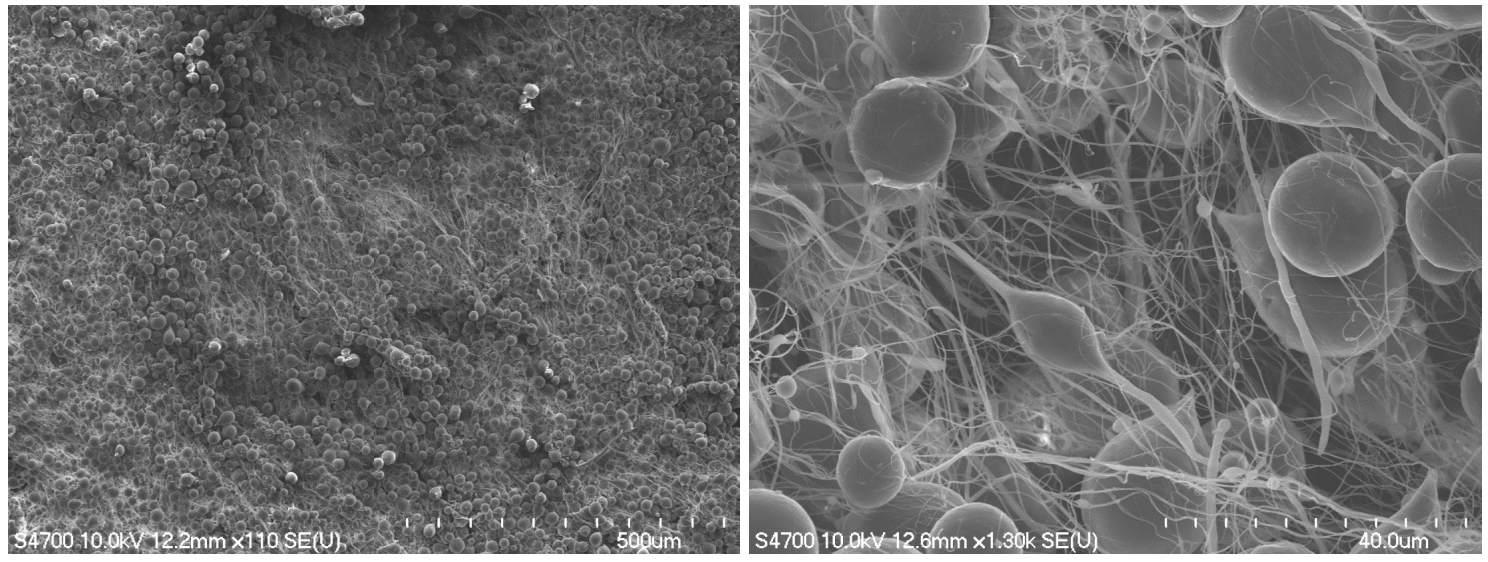

Figure S8. High magnification Field emission scanning electron microscopic (FESEM) images of the PCL-PANI-24kV scaffolds. Beading was present throughout the sample and without any localization of fibers to form structures. 

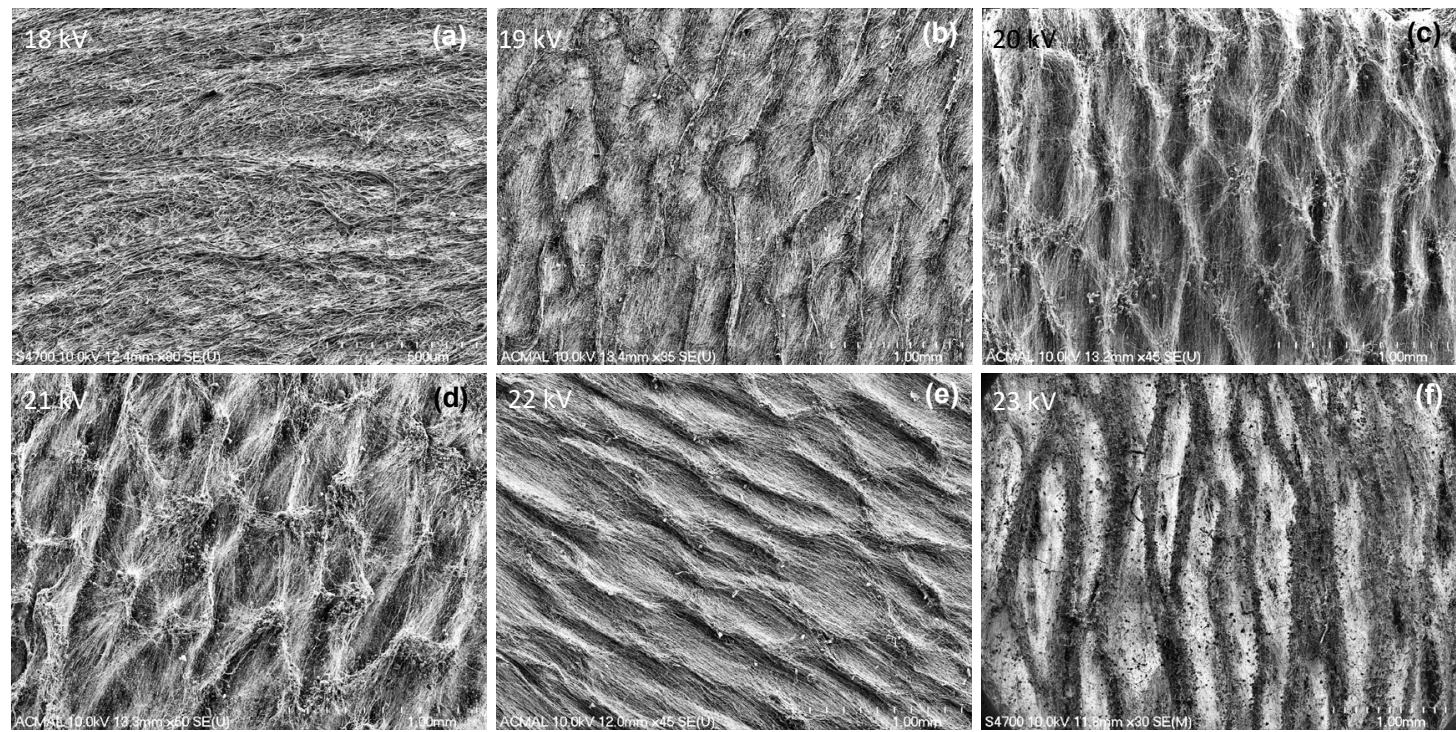

Figure S9. Field emission scanning electron microscopic (FESEM) images of the PCLPANI scaffolds exhibiting porous morphology and fiber alignment. The high contrast FESEM images reveal the 3D honeycomb structures.

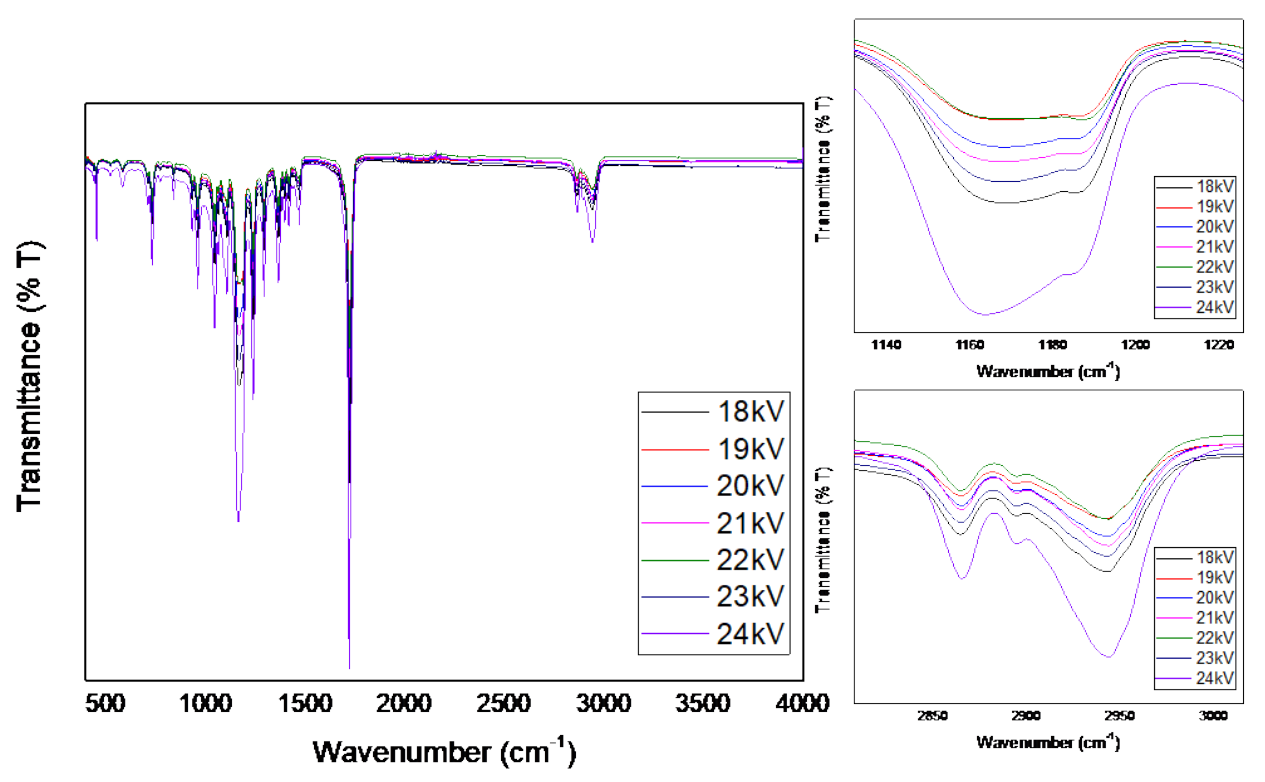


Figure S10. Surface analysis of the different scaffolds illustrated by the ATR-FTIR spectra. There was no significant difference between the different peaks for all the scaffolds indicating no significant difference in surface chemistries of the scaffolds.
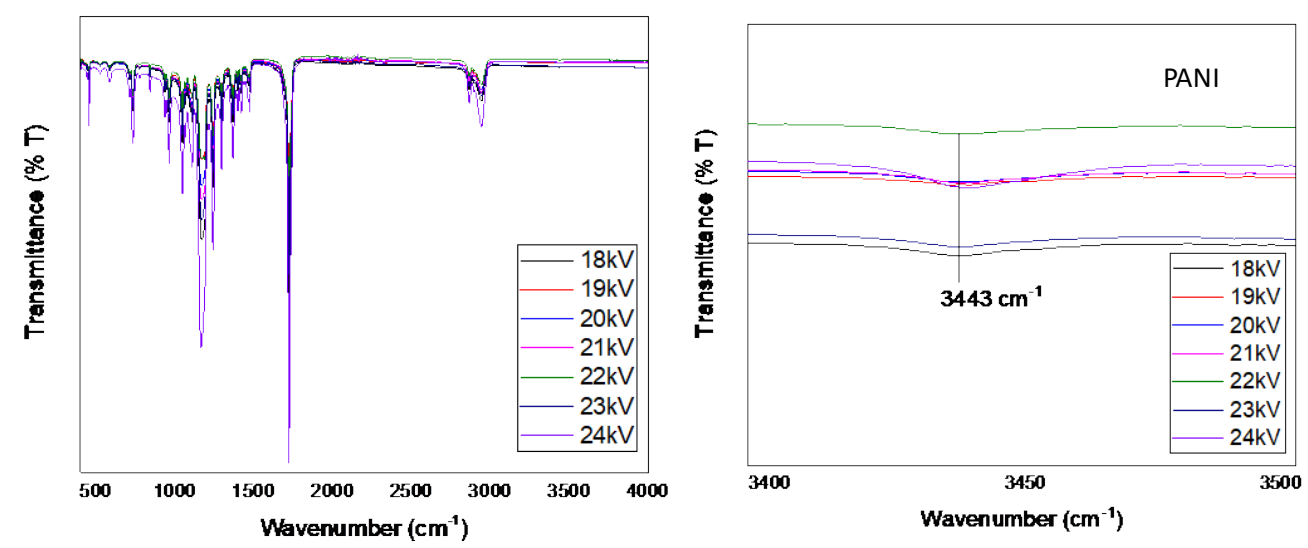

Figure S11. Surface analysis of the different scaffolds illustrated by the ATR-FTIR spectra. The characteristic peak corresponding to aniline can be seen at $3443 \mathrm{~cm}^{-1}$. The intensity of transmittance is relatively lower than the other peaks. The peaks have relatively low intensity because of the low concentration of the bonds in the scaffold. 


\title{
$4 \quad$ Engineered three-dimensional scaffolds modulating fate of breast cancer cells using stiffness and morphology related cell adhesion
}

\author{
Samerender N. Hanumantharao ${ }^{1}$, Carolynn A. Que ${ }^{1}$, Brennan J. Vogl ${ }^{1}$, ${ }^{*}$ Smitha Rao ${ }^{1}$, \\ Senior Member, IEEE \\ ${ }^{1}$ Department of Biomedical Engineering, Michigan Technological University, Houghton, \\ MI 49931, USA
}

Published in: IEEE Open Journal of Engineering in Medicine and Biology ( Volume: 1 )

Page(s): $41-48$

Date of Publication: 09 January 2020

Electronic ISSN: 2644-1276

INSPEC Accession Number: 19425089

DOI: $10.1109 / \mathrm{OJEMB} .2020 .2965084$

Publisher: IEEE

*Corresponding author:Email: smithar@mtu.edu

Abstract - Goal: Artificially engineering the tumor microenvironment in vitro as a vital tool for understanding the mechanism of tumor progression. In this study, we developed three-dimensional cell scaffold systems with different topographical features and mechanical properties but similar surface chemistry. The cell behavior was modulated by the topography and mechanical properties of the scaffold. Adenocarcinoma (MCF7), triplenegative (MDA-MB-231) and premalignant (MCF10AneoT) breast cancer cells were seeded on the scaffold systems. The cell viability, cell-cell interaction and cell-matrix interactions were analyzed. The preferential growth and alignment of specific population of cells were demonstrated. Among the different scaffolds, triple-negative breast cancer cells preferred honeycomb scaffolds while adenocarcinoma cells favored mesh scaffolds and premalignant cells preferred the aligned scaffolds. The 3D model system developed here can be used to support growth of only specific cell populations or for the growth of tumors. This model can be used for understanding the topographical and mechanical features affecting tumorigenesis, cancer cell growth and migration behavior of malignant and metastatic cancer cells. 
Index Terms - 3D scaffold, breast cancer, cell adhesion, microenvironment, durotaxis, topotaxis.

\section{Impact Statement:}

A standard ready to use template-free 3D scaffold tailored to mimic the durotactic and topotactic gradients of breast cancer cell for in vitro tumor modeling.

\subsection{Introduction}

The cancer microenvironment is a complex system consisting of extracellular matrix, stromal cells, adipocytes, fluids and vasculature [237]. This system is dynamically remodeled during tumorigenesis leading to a constantly evolving temporal and spatial $3 \mathrm{D}$ structures with distinct physical and pathophysiological alterations conducive to tumors [238]. The conventional 2D cell culture systems do not recapitulate the $3 \mathrm{D}$ tumor microenvironment. The cells grow in monolayers, lose polarity, and have an altered shape leading to changes in gene expression and splicing [239-243]. It fails to recreate the complex 3D intercellular signaling cascades and cell-matrix interactions, hypoxic conditions characteristic of tumor microenvironment, and communication between cells in different niches [244-246]. Three-dimensional systems such as tumor cell spheroids lack repeatability and are difficult to handle for animal studies [247]. The scaffolds provide a stable 3D environment for the cells to adhere, migrate, proliferate and differentiate[248]. They closely mimic the microenvironment with hypoxia-like conditions and cellular niches. Various materials, both natural (e.g. Engelbreth-Holm-Swam extract, collagen) and synthetic (polycaprolactone, poly(lactic-co-glycolic acid) have been used to fabricate scaffolds [249-251]. Recently, the adverse impact of using biological materials on immune cell recruitment was reported by Wolf and colleagues [252]. Synthetic polymer scaffolds have an advantage of being readily available and their production can be upscaled industrially [253]. Current 3D scaffold systems have limitations in design and connecting in vivo and in vitro conditions due to reductionist approaches. The synthetic material systems provide key information regarding cell migration and signaling cascades, but fail to consider the mechanical properties and topographical cues, including roughness, curvature, porosity and fibrosity of the tumor microenvironment [254-258].

In this study, we engineered 3D scaffolds composed of well-defined morphologies and mechanical properties from polycaprolactone (PCL) using electrospinning [259, 260]. PCL is a synthetic, biodegradable, aliphatic polyester with slow and controllable degradation rates, and tunable mechanical properties [261]. Scaffolds with mesh, aligned and honeycomb morphologies (Fig. 1) were fabricated by manipulating the parameters used during electrospinning. Scaffolds with mesh morphology were designed to mimic the fibrous structure naturally present in the extracellular matrix of the breast tissue (ECM). The aligned morphology (naturally present in connective tissue) in scaffolds has previously been demonstrated to provide cues for durotaxis leading to differentiation, alignment of cells and as a predictor for breast cancer survival [262-266]. The porous honeycomb structure (naturally present in bones and alveolar tissue) mimicking the complex 
architecture present in tissues has been previously explored for tissue engineering [267269], fibroblast growth and to inhibit the growth of cancer cells [270, 271]. Here we further investigate and delineate the effect of scaffold morphology on cancer cells. Breast cancer cell lines representing ductal adenocarcinoma (MCF-7), triple-negative metastatic (MDAMB-231) and pre-malignant cancer (MCF10AneoT) were used to investigate the role of mechanical properties and topography on cancer cell adhesion and proliferation, and provide insights into the preferential behavior of cancer cells [272]. The scaffolds replicating different morphologies naturally present in the body helps in mimicking the conditions in vitro and explore the potential of topotactic and durotactic gradients of the extracellular matrix.

\subsection{Materials and methods}

\subsubsection{Fabrication of Scaffolds}

All the materials were used as procured unless specified otherwise. Polycaprolactone (PCL): (Mw 70,000 GPC; Scientific Polymer Products, USA) was used to obtain a sol-gel consisting of $20 \%$ PCL in chloroform (Sigma Aldrich, USA) for electrospinning. The voltage, rotational speed of the rotating collector and the polymer feed rate were varied as indicated in table 1 (EM-DIG and EM-RTC; IME Technologies, Netherlands). Humidity, temperature, polymer fluid volume and tip-collector distance were constant.

Table 1. Parameters Used for Electrospinning Scaffolds

\begin{tabular}{cccc}
\hline \hline Type of Scaffold & $\begin{array}{c}\text { Voltage } \\
(\mathrm{kV})\end{array}$ & $\begin{array}{c}\text { Rotational } \\
\text { Speed (RPM) }\end{array}$ & $\begin{array}{c}\text { Polymer Feed Rate } \\
(\mu \mathrm{L} / \mathrm{min})\end{array}$ \\
\hline Mesh & 11 & 150 & 4 \\
Aligned & 11 & 275 & 4 \\
Honeycomb & 10 & 300 & 3
\end{tabular}

\subsubsection{Characterization of Scaffolds}

The scaffolds were prepared by sputter coating with a $5 \mathrm{~nm}$ thick coating of $\mathrm{Au} / \mathrm{Pd}$ for field emission scanning electron microscope (FESEM; Hitachi S-4700 FE-SEM). Fiji[273] was used for image analysis. The surface chemistry of the scaffolds was characterized using Attenuated Fourier Transform Infrared Spectroscopy (ATF-FTIR, Thermo Scientific ${ }^{\mathrm{TM}}$, Nicolet ${ }^{\mathrm{TM}}$ iS50) with a deuterated triglycine sulfate detector element. The measurements range of $400-4000 \mathrm{~cm}^{-1}$ at a resolution of $4 \mathrm{~cm}^{-1}$ with 256 scans was used. The mechanical properties of the scaffolds were determined by using a dynamic mechanical analyzer (TA Instruments ${ }^{\mathrm{TM}}$, DMA Q800) under uniaxial strain ramp at isothermal conditions $\left(37^{\circ} \mathrm{C}\right)$. 
The Young's modulus was determined from the linear region of the stress-strain plot, uniaxial stiffness was determined from the force-displacement curve. The modulus of toughness and modulus of resilience were calculated from the area under the curve and area under the linear region of the stress-strain curve, respectively.

\subsubsection{Cell Culture, Seeding, Viability, and Immunochemistry}

Breast ductal adenocarcinoma cancer cells (MCF7/ATCC ${ }^{\circledR}$ HTB-22 ${ }^{\mathrm{TM}}$ ) and triplenegative malignant basal breast cancer cells (MDA-MB-231/ATCC® HTB-26 ${ }^{\mathrm{TM}}$ ) were procured from American Type Cell Culture (ATCC). The premalignant cancer cell line, MCF10AneoT, was acquired from the Animal Model and Therapeutics Evaluation Core (AMTEC), Barbara Ann Karmanos Cancer Institute, Wayne State University. All cells were maintained under standard culture conditions and seeded on scaffolds as previously reported [33]. The scaffolds of $0.25 \mathrm{~cm}^{2}$ and $0.5 \mathrm{~cm}^{2}$ area (1500 cells) were used for cell viability and immunocytochemistry respectively following sterilization in ethanol and irradiation in UV. The cell viability $(\mathrm{n}=9)$ was analyzed using CellTiter-Blue ${ }^{\circledR}$ Cell Viability Assay (Promega, Madison, WI). Cells on tissue culture treated plates in similar conditions served as positive controls $(\mathrm{n}=3)$. The fluorescence intensity was measured after 4 hours (Beckman Coulter DTX 880 Multimode Detector, ex/em 560nm/590nm). Fixed samples were permeabilized with Triton-X 100 and stained with Alexa Fluor ${ }^{\circledR} 594$ Phalloidin (Invitrogen, USA) and DAPI (4',6-diamidino-2-phenylindole) (Life Technologies, USA) for visualizing the cytoskeletal F-Actin and A-T regions of the nucleus, respectively, according to the manufacturer's protocols.

\subsubsection{Statistical Analysis}

Mechanical characterization of the scaffold was represented as mean \pm SD (standard deviation). For cell viability, descriptive statistics was represented as mean \pm SEM (standard error of mean). OriginPro $2018 \mathrm{~b}$ and IBM ${ }^{\circledR}$ SPSS statistics V25 was used for statistical evaluation of cell proliferation. One-way ANOVA followed by post-hoc Tukey's HSD test was used to calculate significance $(\mathrm{p}<0.05)$ between days and difference between cell lines for each scaffold morphology. 


\subsection{Results}

\subsubsection{Topographical Characterization}
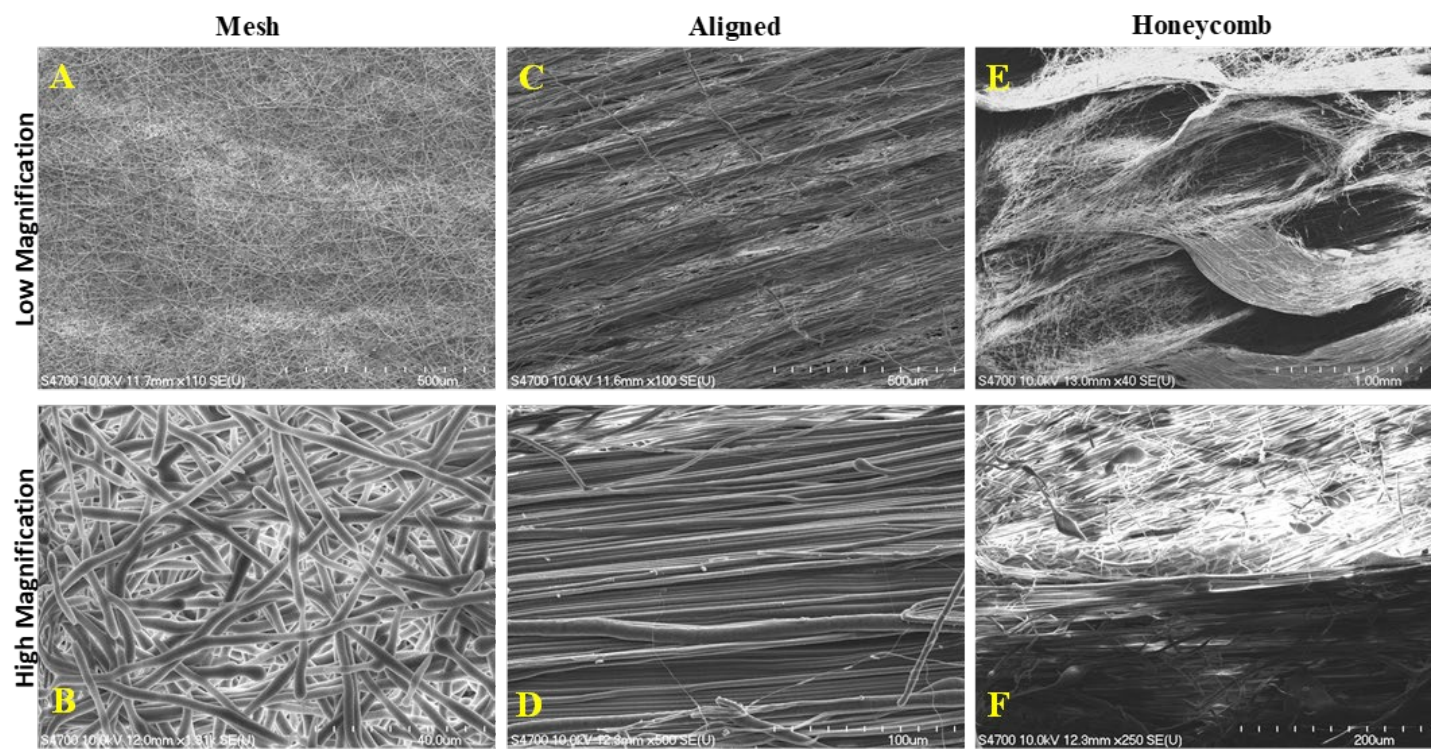

Fig. 1. Field emission scanning electron microscopic (FESEM) images of the PCL scaffolds exhibiting different morphologies. The low magnification images (A,C,E) are present on the top while the high magnification images $(B, D, F)$ are present in the bottom.

From the FESEM images (Fig. 1), the formation of three distinct topographies can be inferred. The mesh scaffolds (Fig. 1A) have randomly oriented fibers, densely packed forming a 3D structure. The change in contrast (Fig. 1B) of the mesh like network indicates different layers. The aligned scaffolds had fibers tightly packed along an identical orientation. The scaffolds exhibited alternating regions of larger and smaller diameter fibers (Fig. 1C). A high concentration of overlapping aligned fibers in a tight network is visible in the high magnification (Fig. 1D). A low magnification image of the honeycomb scaffolds (Fig. 1E) composed of interlocking fibers in a specific pattern forming asymmetrical elongated honeycomb like structures. The structures had a long-range order and high aspect ratio. Densely packed fibers along the walls and aligned fibers at the bottom is visible in the high magnification image (Fig. 1F) of the boundary of the pores. The degree of alignment was the lowest in the mesh scaffolds (Fig. 2A) and highest in aligned scaffolds (Fig. 2B). The honeycomb morphology had a degree of alignment spread over a broad range of angles and a higher alignment of fibers than mesh but less than the aligned morphology (Fig. 2C). The fibers in mesh morphology had little depth with uniform topography (Fig. 2D), while the aligned morphology had fibers with a pattern resembling grids forming grooves (Fig. 2E). The porous structures were well defined and present throughout the honeycomb morphology forming distinct regions (Fig. 2F). 

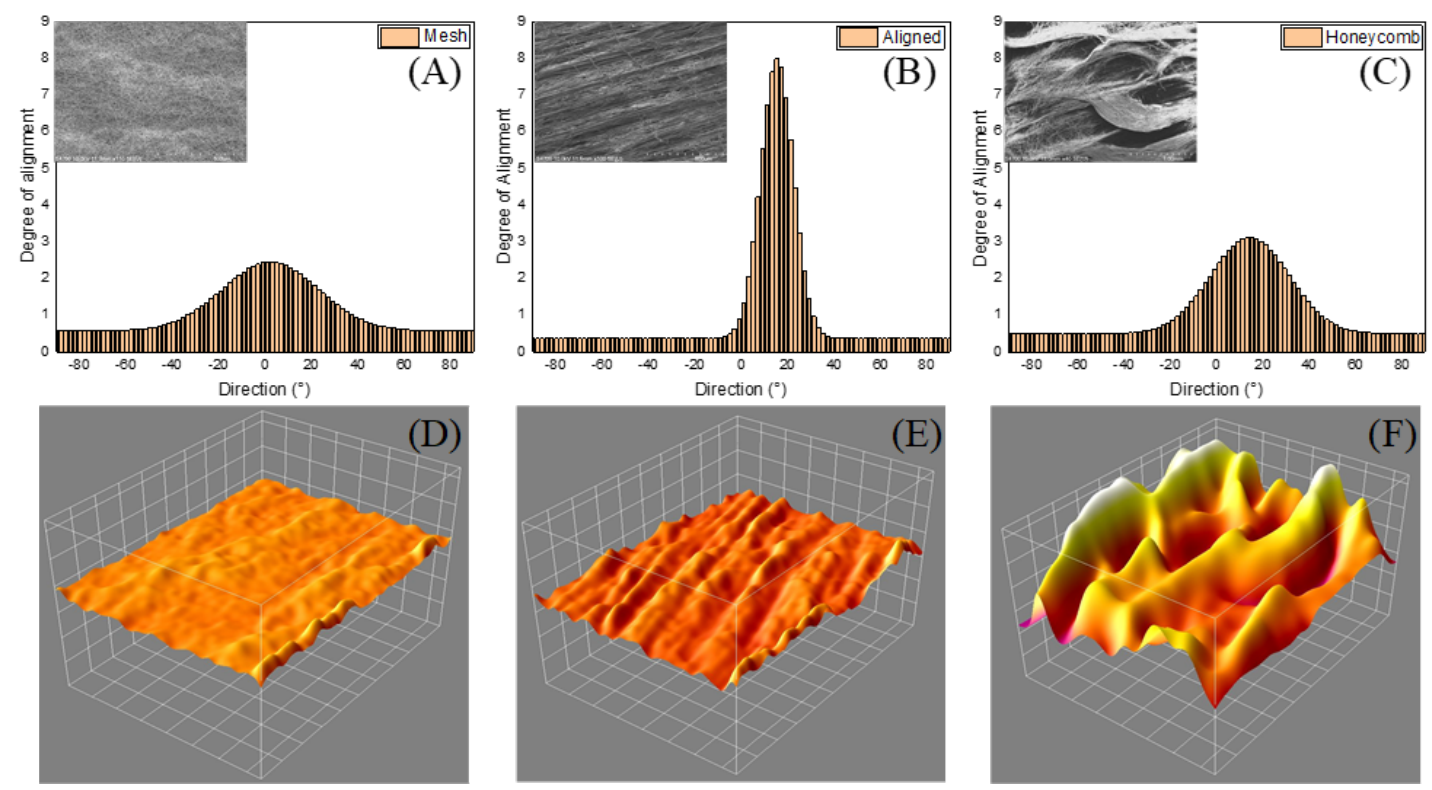

Fig. 2. The degree of alignment and 3D topography scan of different morphologies of the scaffolds was characterized using the directionality plugin in ImageJ from the FESEM images used in figure 1 as seen in the inlay $(n=5)$. A) The mesh fibers had a high dispersion of fibers with low degree of alignment. B) The aligned fibers had a high concentration of fibers in a narrow angle range with little deviation in other directions. C) The honeycomb scaffolds had a broader range of deviation compared to the aligned scaffold but a much narrower distribution than the mesh scaffolds. D) The fibers in mesh had little depth profile. E) The fibers in aligned morphology had aligned fibers forming a pattern resembling grids F) The fibers in the honeycomb morphology had well defined porous structure forming distinct regions.

\subsubsection{Surface Chemistry}
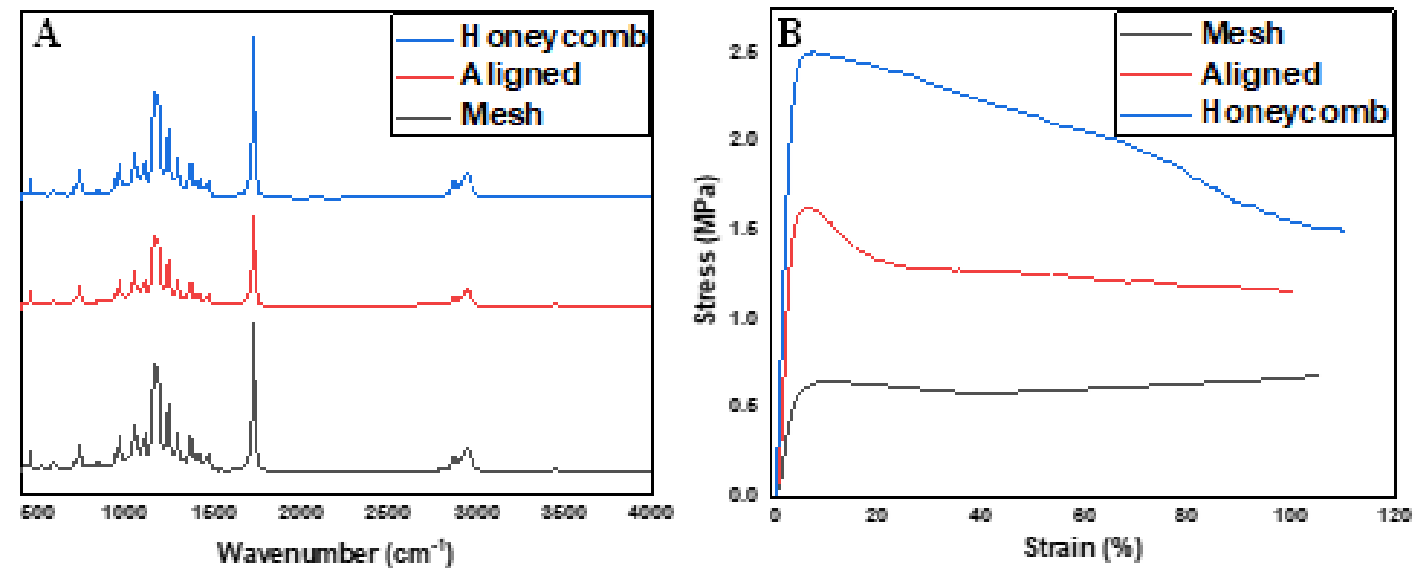
Fig. 3. The surface and mechanical characterization of the PCL scaffolds of different morphologies was done. A) Surface characterization was done using ATR-FTIR spectroscopy. The peaks distinctive to the molecular bond orientations present in PCL were identified. All the morphologies had similar surface chemistry. B) The mechanical properties of the scaffolds was characterized using DMA at isothermal conditions $\left(37^{\circ} \mathrm{C}\right)$ and represented as stress-strain graph.

Surface characterization of the chemical bonds on the scaffolds was done using ATR-FTIR (Fig. 3A and S1). As the incident beam was focused on a larger surface area, it cannot be used to compare between the isotropic nature of the fibers in different scaffold morphologies. The stretching of the $\mathrm{C}-\mathrm{O}$ and $\mathrm{C}-\mathrm{C}$ bonds in the crystalline phase causes a peak at $1294 \mathrm{~cm}^{-1}$. The high electric field applied causes the PCL chains to orient along a direction accentuating the crystalline phase of PCL[274]. The peaks at $2942 \mathrm{~cm}^{-1}$ and $2865 \mathrm{~cm}^{-1}$ represent asymmetric and symmetric stretching of the $\mathrm{CH}_{2}$ group. The peak at $1723 \mathrm{~cm}^{-1}$ corresponds to $\mathrm{C}=\mathrm{O}$ vibration of ester. The bands at $1239 \mathrm{~cm}^{-1}$ and $1165 \mathrm{~cm}^{-1}$ are associated with asymmetric and symmetric stretching of the ester $\mathrm{COO}$ group. The peak at $1365 \mathrm{~cm}^{-1}$ correspond to the $\mathrm{CH}_{2}$ band vibrations while the $\mathrm{O}-\mathrm{C}$ vibrations and $\mathrm{CH}_{2}$ vibration occur at $961 \mathrm{~cm}^{-1}$ and $732 \mathrm{~cm}^{-1}$ respectively.

\subsubsection{Mechanical Properties}

Table 2. Mechanical properties of the different morphologies of the scaffold

\begin{tabular}{lccc}
\hline \hline & Mesh & Aligned & Honeycomb \\
\hline $\begin{array}{l}\text { Young's Modulus } \\
\text { (MPa) }\end{array}$ & $0.155 \pm 0.01$ & $0.560 \pm 0.12$ & $0.569 \pm 0.14$ \\
$\begin{array}{l}\text { Modulus of } \\
\text { Resilience }\end{array}$ & $1.603 \pm 0.64$ & $2.945 \pm 0.59$ & $5.547 \pm 0.04$ \\
$\begin{array}{l}\text { Ultimate Tensile } \\
\text { Strength (MPa) } \\
\text { Strain at Failure } \\
\quad \text { (\%) }\end{array}$ & $0.818 \pm 0.12$ & $1.549 \pm 0.07$ & $2.341 \pm 0.14$ \\
& $104.512 \pm$ & $106.736 \pm 5.99$ & $107.512 \pm 4.60$ \\
$\quad \begin{array}{ccc}\text { Modulus of } \\
\text { Toughness }\end{array}$ & $76.473 \pm$ & $136.885 \pm 9.41$ & $189.273 \pm$ \\
& 10.63 & & 28.40 \\
\hline \hline
\end{tabular}

The mechanical characterization of the scaffolds (Table 2) was done using DMA at isothermal conditions (Fig. 3B). The stress-strain behavior of the scaffolds was unique to each morphology. The honeycomb scaffold has the highest average ultimate strength and 
stiffness. The aligned scaffolds have mechanical properties comparable to the honeycomb scaffolds with respect to the Young's modulus and stiffness, however the modulus of toughness is lower than honeycomb scaffolds. The mesh scaffolds have relatively poor strength and toughness. All three morphologies have a non-significant difference in strain at failure.

\subsubsection{Immunocytochemistry}

Breast cancer cell lines representing various stages of cancer progression (adenocarcinoma, premalignant, triple-negative/metastatic) were used to evaluate the behavior of cancer cells on different topographies and mechanical properties of the scaffold. The cells were stained and fixed on days 1,2 and 3. Qualitative analysis of the behavior of the cells to changes in morphology of the scaffold was assessed by immunocytochemistry on fixed cells on days 1,2 , and 3 after seeding. High magnification images of some of the phenotypes used for characterizing the behavior of the scaffolds is shown in figure S2.

\subsubsection{MCF7}
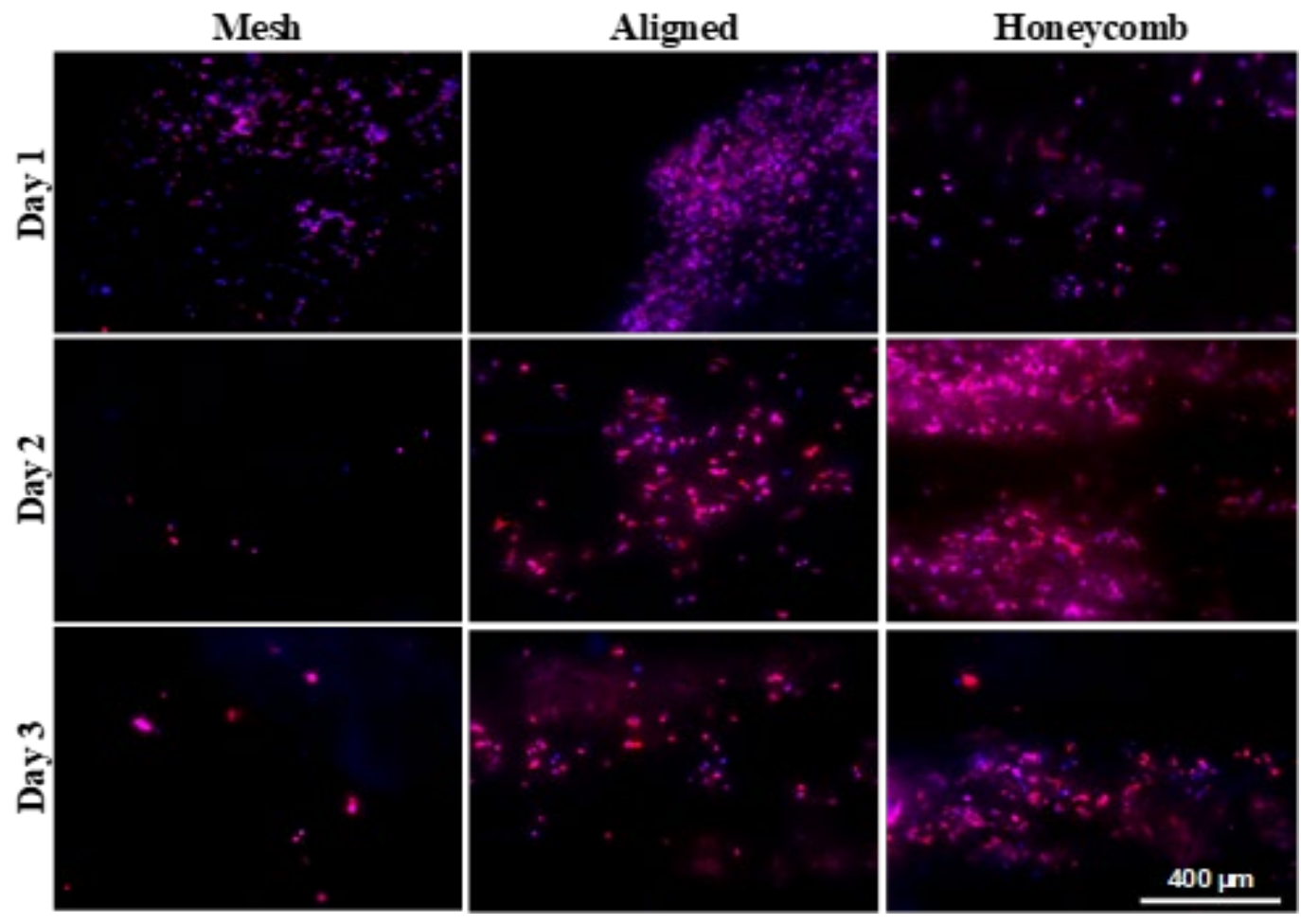

Fig. 4. Fluorescent microscope images of Adenocarcinoma cells (MCF7) on different morphologies of the PCL scaffold on days 1, 2 and 3. The nuclei were stained with DAPI (blue) and the F-actin filaments were stained with Alexa Fluor ${ }^{\circledR} 594$ Phalloidin (red). The overlapping of the blue and red and dispersion by the fibers causes some of the cells to be seen pink in color. Images captured at 10X magnification 
From Fig. 4 and S3, the cells were clumped on all three morphologies on day 1 with extensive clumping in the aligned scaffolds. In the mesh morphology, on days 2 and 3 , cells infiltrated the scaffold and were spread affecting imaging. In the aligned scaffold, on days 2 and 3 oriented along the direction of alignment of the fibers and were spread out with an elongated morphology. The honeycomb scaffold had a high concentration of cells in the pores and almost negligible number of cells on the boundary of the pores on day 2 . On day 3, the cells infiltrated the layers of fibers and were present in between the fibers (blurred regions between the fibers). Based on the cellular distribution, morphology and orientation (day 3), it appears that the cells responded to the nanotopographical cues, distinctive of the morphologies of the scaffolds.

\subsubsection{MDA-MB-231}
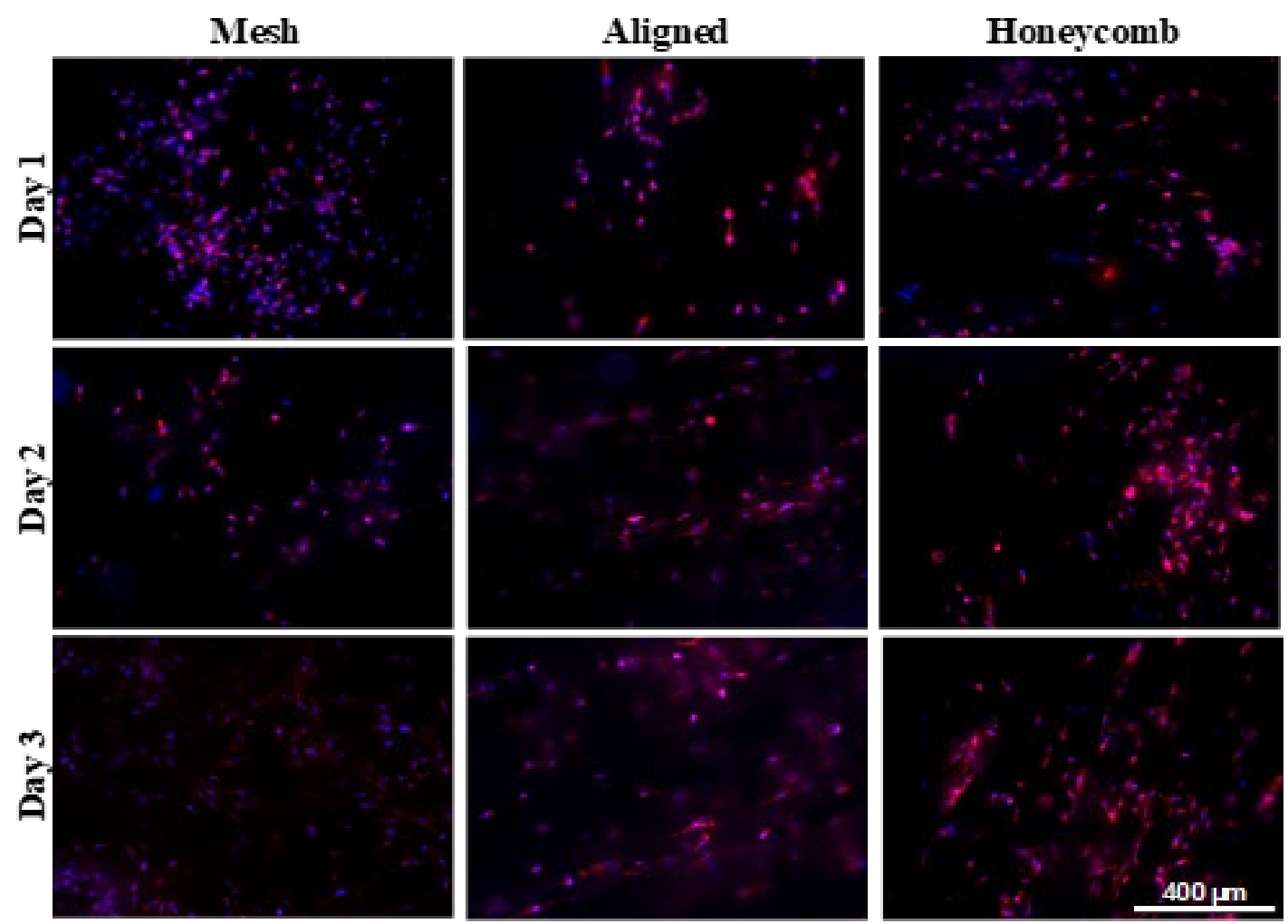

Fig. 5. Fluorescent microscope images of triple negative breast cancer cells (MDA-MB231) on different morphologies of the PCL scaffold on days 1, 2 and 3. The nuclei were stained with DAPI (blue) and the F-actin filaments were stained with Alexa Fluor ${ }^{\circledR} 594$ Phalloidin (red). The overlapping of the blue and red and dispersion by the fibers causes some of the cells to be seen pink in color. Images captured at $10 \mathrm{X}$ magnification.

From Fig. 5 and S4, the cells were distributed across the mesh scaffold without any orientation on all three days. In the aligned scaffold, the cells lacked alignment on day 1, but were spread out. Cell alignment and elongation was along the fiber alignment on day 2 . On day 3 , the cells infiltrated the layers (blurred background) on day 3 . There was little 
cellular alignment in the honeycomb on day 1 . However, this improved on day 2 and the cells infiltrated the scaffolds with preferential attachment to the walls rather than the underlying layers of the pores. Clumping was not observed in any of the morphologies.

\subsubsection{MCF10Aneot}
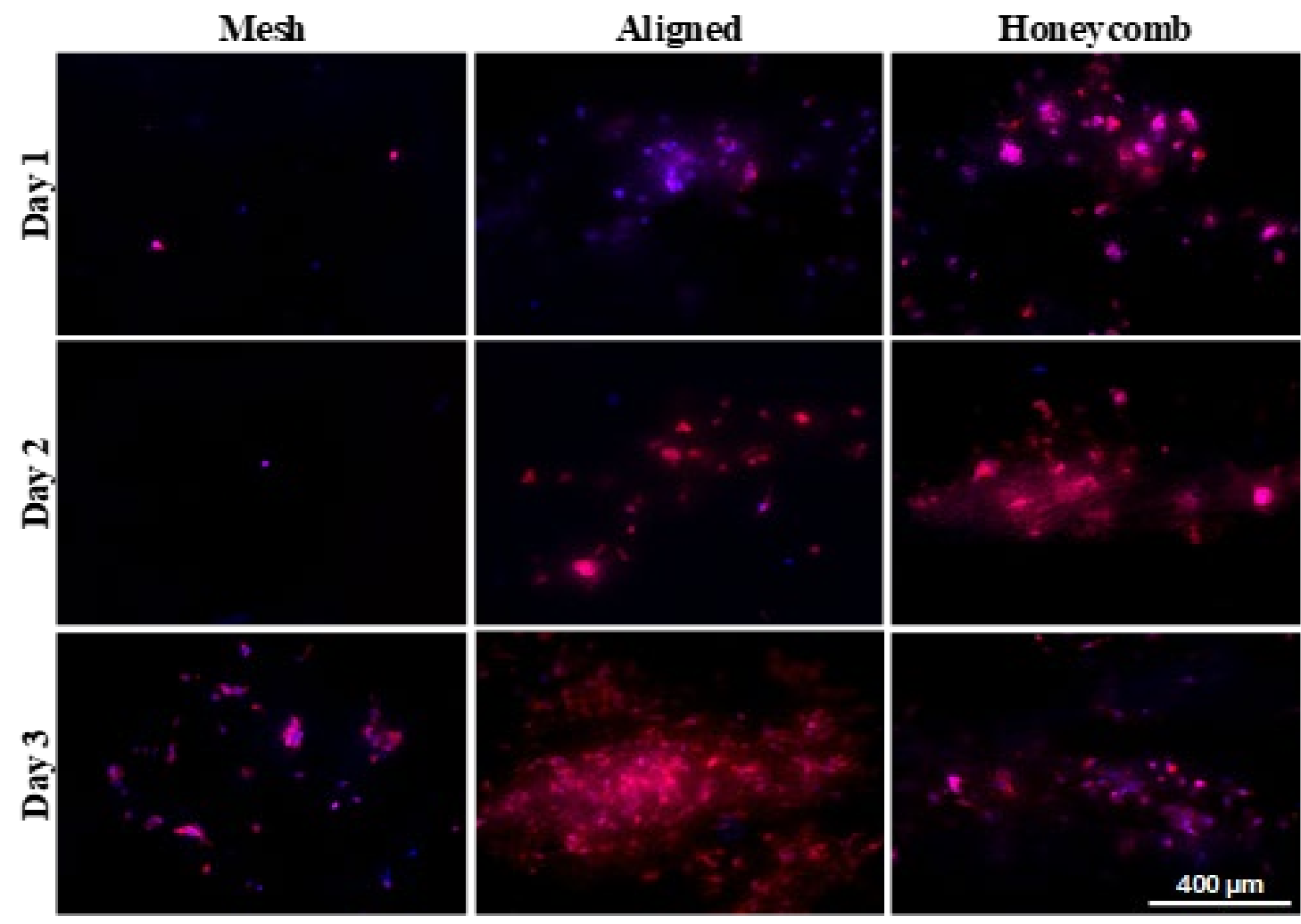

Fig. 6. Fluorescent microscope images of premalignant breast cancer cells (MCF10AneoT) on different morphologies of the PCL scaffold on days 1, 2 and 3. The nuclei were stained with DAPI (blue) and the F-actin filaments were stained with Alexa Fluor ${ }^{\circledR} 594$ Phalloidin (red). The overlapping of the blue and red and dispersion by the fibers causes some of the cells to be seen pink in color. Images captured at 10X magnification.

From Fig. 6 and S5, the cells appear clumped in all the scaffold morphologies for all days with infiltration. In the mesh scaffold, the clumping was localized and lacked cellular orientation. In the aligned scaffolds, the cells were spread out with minimal cellular alignment on days 1 and 2 but, appear to align along the fibers on day 3 . In the honeycomb scaffold, cell infiltration was observed after day 1, with increased infiltration on days 2 and 3 . The cells on day 3 did not present the clumping observed on day 2 . 


\subsubsection{Cell Viability}
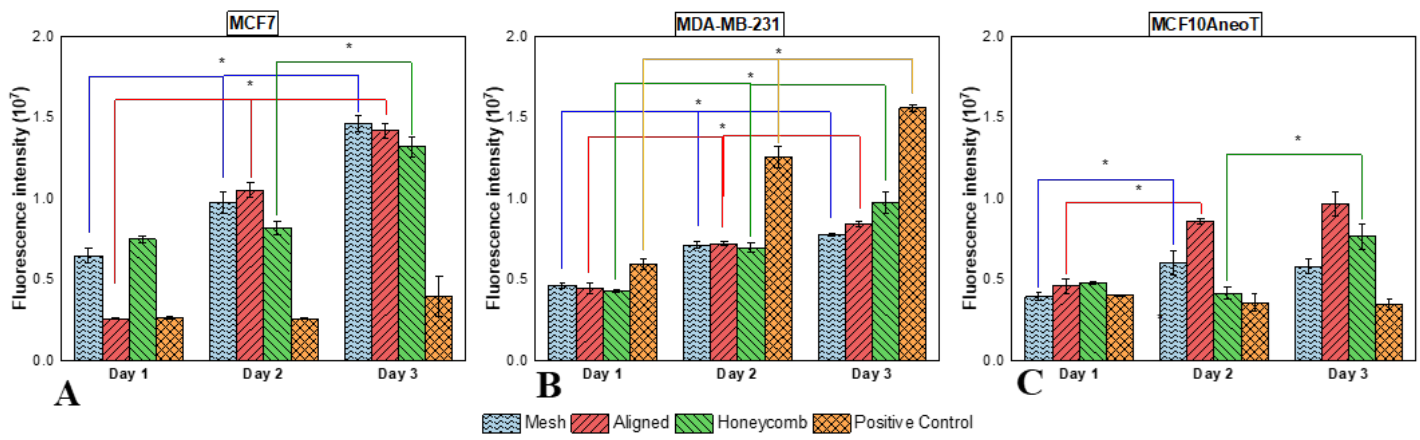

Fig. 7. Quantification of cell viability on days 1,2 and 3 on different morphologies and cell lines. A) Cell viability of MCF7 (adenocarcinoma) cells on different morphologies of the scaffold. Cells seeded on mesh scaffold had a significant increase in cell number till day 3 . B) Cell viability of MDA-MB-231 (triple negative) cells on different morphologies of the scaffold. The cell number increased consistently on all scaffolds till day 3. C) Cell viability of MCF10AneoT (premalignant) cells on different morphologies of the scaffold. The increase in cell viability was inconsistent across any morphology. Error bars represent the standard error of mean and statistical significance is indicated by $\mathrm{p}$ values $(* \mathrm{p}<0.05)$

The cell viability on scaffolds on days 1, 2 and 3 were analyzed by characterizing the reduction of resazurin to resorufin. MCF7 had a significant increase in cell number across all scaffold morphologies on all days (Fig. 7A). The number of viable cells was lowest in the positive control with non-significant increase for each day. While the MDA-MB-231 cells had a statistically significant increase in cell viability over all the days on all the morphologies (Fig. 7B), the positive control was the most favorable. The viability of MCF10AneoT cells (Fig. 7C) was not uniform. The increase in cell number was more pronounced from day 1 to day 2 in the mesh and aligned scaffolds and from day 2 to day 3 in honeycomb scaffolds with non-significant increase in the positive control. The increase in cell number for MDA-MB-231 and MCF7 was uniform in all scaffold morphologies.

\subsection{Discussion}

The parameters used for electrospinning were varied to form fibrous scaffolds with different morphologies from the same polymer blend eliminating variability introduced by using different materials or processing. The applied electric field determines the initial elastic stress and the bending instabilities in the jet [275] and to control the spatial deposition of fibers essential in creating the topographical features [276]. The rotational velocity of the collector helps in controlling the orientation, diameter and alignment[277]. However, the critical rotational velocity is determined by the applied average electric field. A lower rotational speed of the collector yields less alignment whereas higher rotational speed yields fibers with orientation perpendicular to the electric field vector. The average electric field was increased along with the rotational speed to fabricate novel three- 
dimensional honeycomb shaped scaffolds. The dense fiber network helps to mimic conditions of tumor-induced angiogenesis as reported by Bauer and colleagues [278, 279].

The mesh scaffolds behave like an elastomer owing to the low rotational velocity of the collector and voltage applied during electrospinning [280]. The aligned scaffolds have better orientation in the microcrystalline regions because of their morphology increasing the Young's modulus of the scaffold [281, 282]. The honeycomb structure by design has superior mechanical properties than other morphologies. This is due to loss in morphology with increasing strain and the fibers aligning in the direction of stress. The cell viability, cell-cell interaction and cell-scaffold behavior were influenced by the topographical features and mechanical properties of the scaffolds. The MCF7 cells proliferated well in all the scaffolds tested without any preference to a particular topographical feature. This agrees with the findings by Chaudhuri et al. on the inhibition of Rho-ROCK-Myosin signaling in malignant cells leading to proliferation of adenocarcinoma irrespective of the topography [283]. However, the cells preferred elastomeric scaffolds with low Young's modulus and stiffness (mesh) over compared to the honeycomb and the positive control. This agrees well with the experimental investigation on mechanical properties of the MCF7 cells through atomic force microscopy measurements by $\mathrm{Li}$ et al.[284] and durotaxis studies by Cavo et al.[285]. The triple-negative cells on the other hand thrived in scaffolds with high matrix stiffness, as expected due to regulation of the YAP (Yes-associated protein)/TAZ (transcriptional coactivator with PDZ-binding motif) and subsequent activation of the Hippo cascade [286]. The stiffness of the positive control (tissue culture plate $\sim 10 \mathrm{GPa}$ ) plays a major role in the cellular viability and agrees well with the findings by Mah et al.[287]. On the honeycomb and aligned scaffolds, the cell alignment and infiltration were guided by the topography and mechanical properties of the scaffold, demonstrating extensive cellular infiltration and alignment. The stiffness of the scaffolds also positively enhances the migration potential of the metastatic cells as reported by Lin et al.[288] and can drive tumor progression through a TWIST1-G3BP2 mechanotransduction pathway [289]. The premalignant cells preferred the aligned scaffolds and infiltrated and aligned along the orientation of the fibers. The topographical cues provided by the aligned scaffolds helps in cell spreading and can also impact tumor progression and metastasis [290]. However, there was a certain amount of clumping in the cells in all the morphologies. This clumping is directly correlated to the metastatic potential of the cells where, the clumped cells form protrusions followed by invasion[291, 292]. The increased viability and spreading of the premalignant cells in stiffer scaffolds are consistent with the observations made by Rubashkin et al.[293]. Thus, it can be concluded that the cells respond to changes in scaffold topography and mechanical properties based on the stage of cancer, effectively providing a suitable in vitro model. The scaffold provides an ideal platform for studying breast cancer metastasis or for localized therapy to inhibit the growth of metastatic cells. The efficient scaffold design also allows the system to be easily adopted for the study and treatment of other cancers through the respective durotaxic and topotaxic gradients [294]. 


\subsection{Conclusion}

Three-dimensional scaffolds with different topographies and mechanical properties were fabricated using electrospinning from polycaprolactone (PCL) with similar surface chemistry. Adenocarcinoma, triple-negative and premalignant breast cancer cells were seeded on scaffolds with different morphologies and characterized. Cell-cell and cellscaffold interaction was qualitatively analyzed and the cell viability across all the days were quantitatively assessed. The triple-negative cells preferred honeycomb scaffolds with higher stiffness and strength, while adenocarcinoma cells proliferated favorably on mesh scaffolds with low elastic modulus and premalignant cells favored aligned scaffolds with high stiffness and greater contact guidance. The current study can be used to design scaffolds which can mimic the tumor microenvironment and for selectively modeling cancer cell population in an in vitro 3D system.

\subsection{Supplementary Materials}

The accompanying supplementary materials includes ATR-FTIR showing similar surface properties for the different scaffolds presented and additional images of cells on scaffolds.

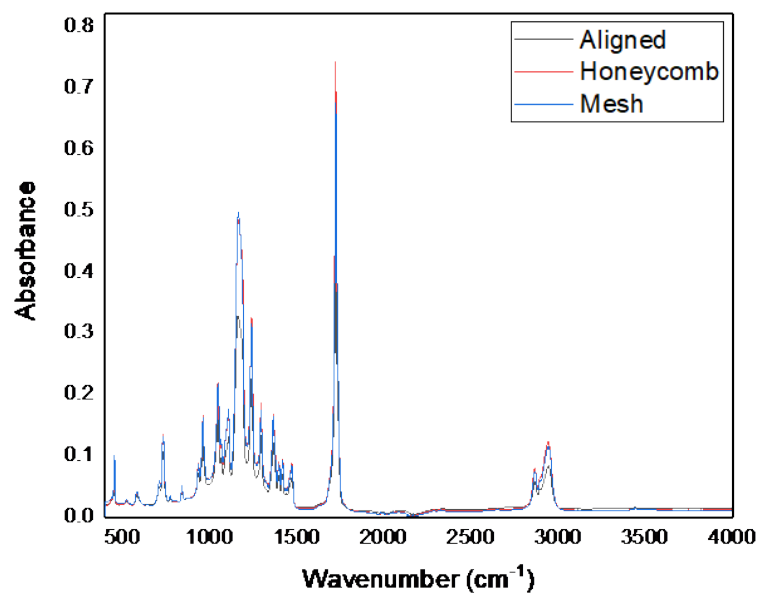

Fig. S1. The surface characterization of the PCL scaffolds of different morphologies was done. The spectrum is overlapped to show the similarity in absorbances of characteristics bonds of PCL from different morphologies of scaffolds. 

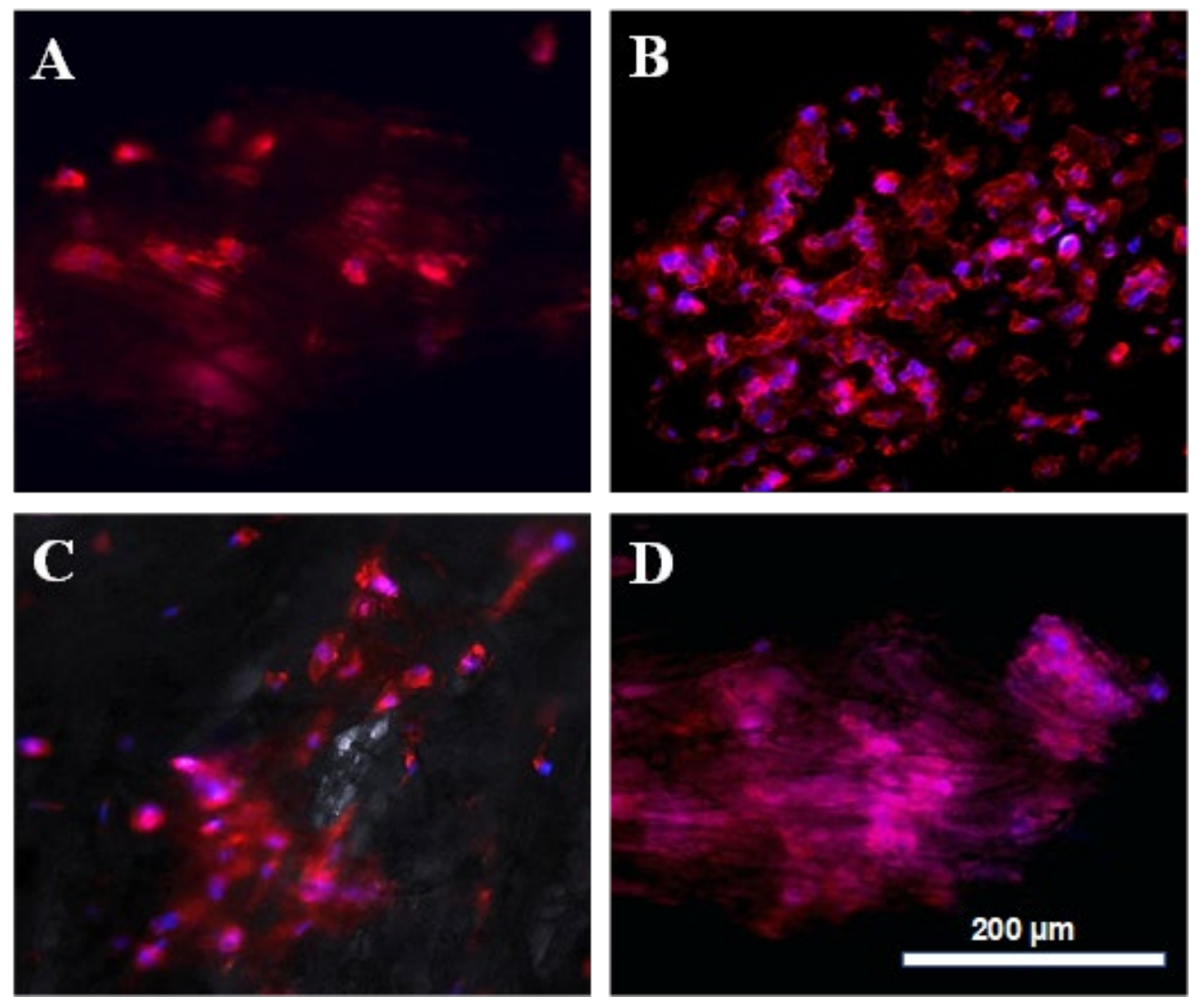

Fig. S2. Phenotypes of the cells on scaffolds used to define cell behavior on the different morphologies of the scaffold. The phase images show the fibers and the overlaid fluorescent images show the alignment of nucleus against the orientation of the fibers. A) Aligned and elongated cells infiltrating into the scaffold. B) Clumped cells on scaffolds C) Cells aligning along the alignment of the scaffold D) The cells infiltrating through the different layers of the scaffolds in clumps. 


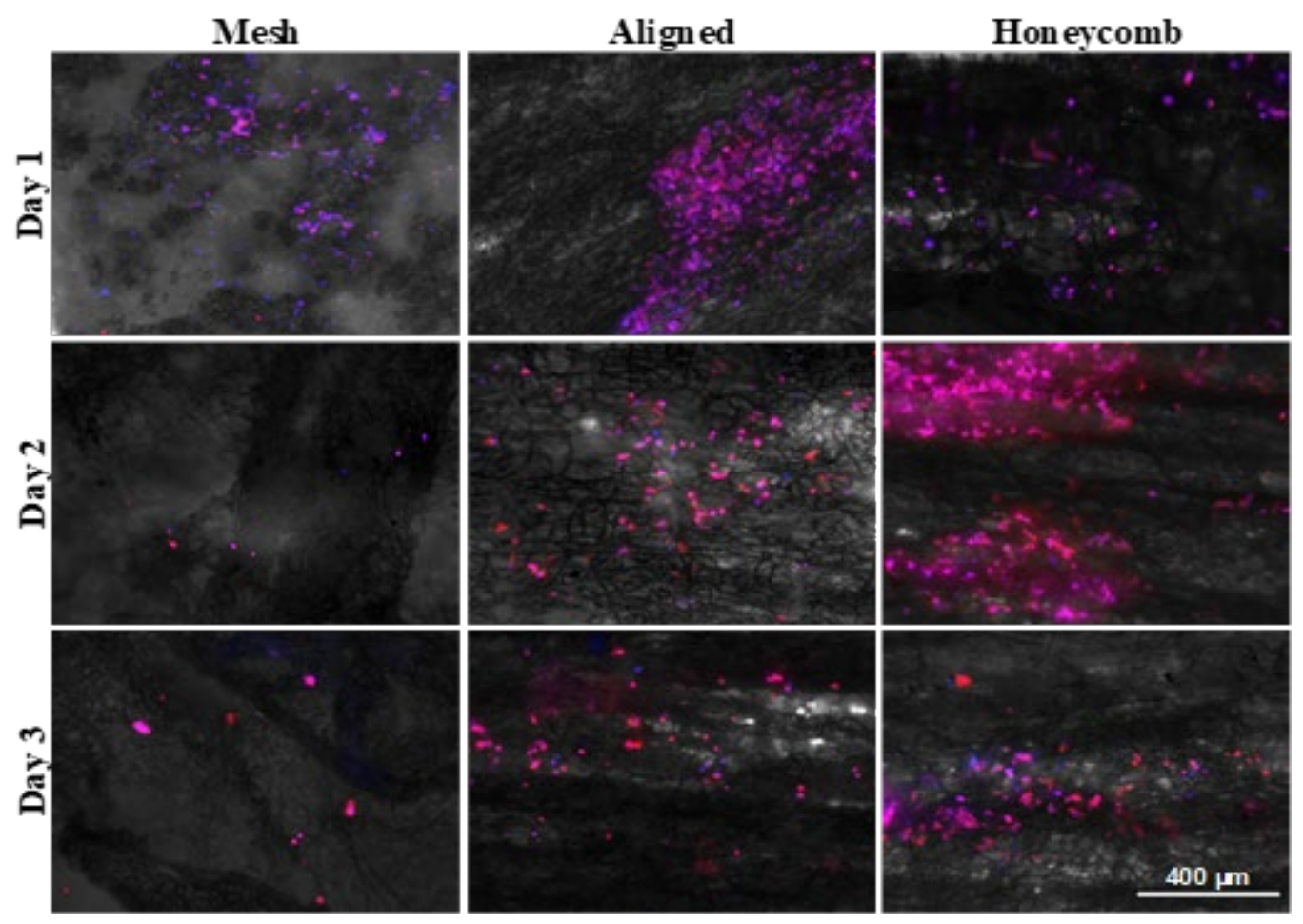

Fig. S3. Fluorescent microscope images of adenocarcinoma cells (MCF7) on different morphologies of the PCL scaffold on days 1, 2 and 3. The nuclei were stained with DAPI (blue) and the F-actin filaments were stained with Alexa Fluor ${ }^{\circledR} 594$ Phalloidin (red). The images were overlaid with the phase contrast image to provide additional information about the scaffold morphology. The overlapping of the blue and red and dispersion by the fibers causes some of the cells to be seen pink in color. Images captured at $10 \mathrm{X}$ magnification. 

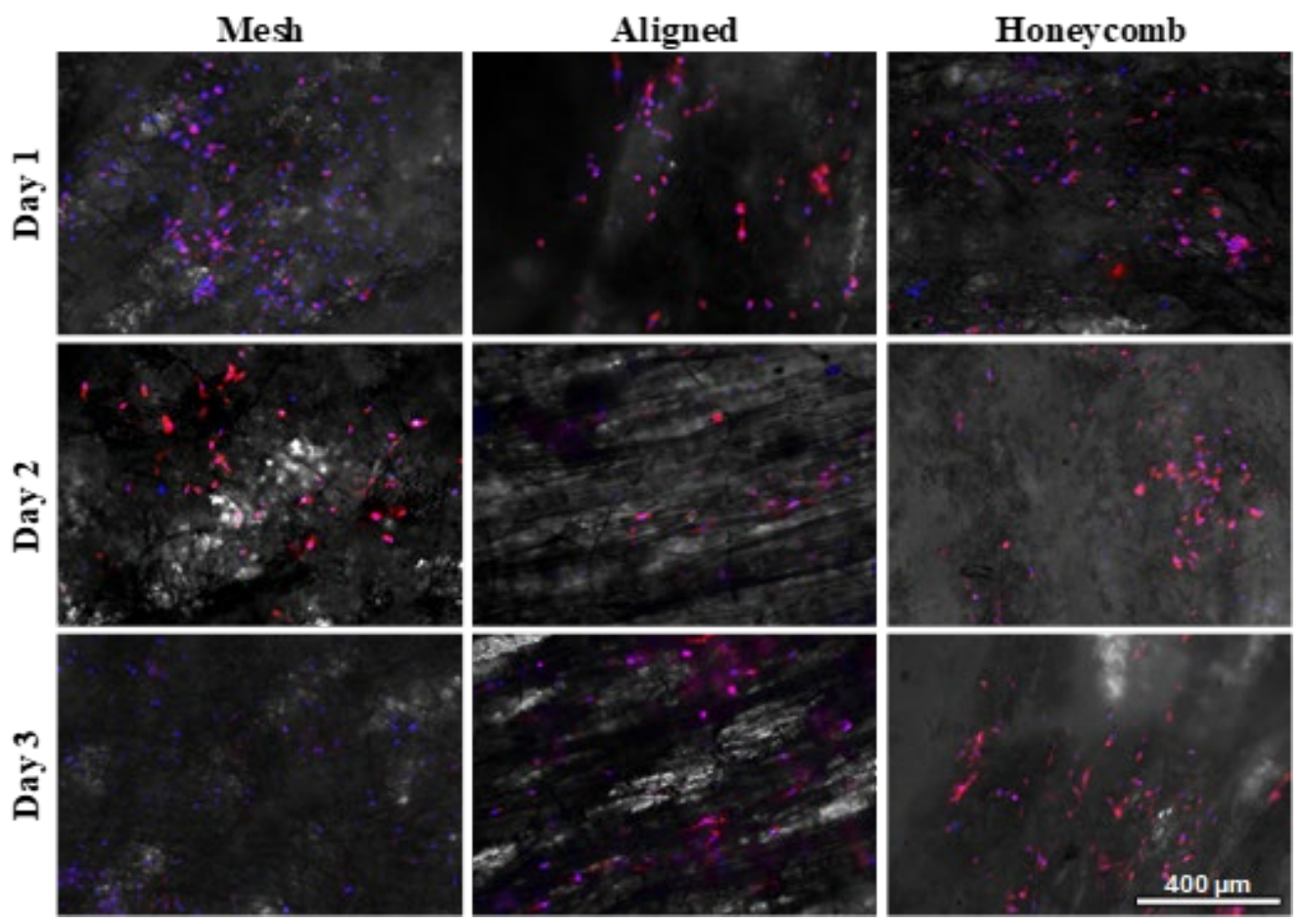

Fig. S4. Fluorescent microscope images of triple negative breast cancer cells (MDA-MB231) on different morphologies of the PCL scaffold on days 1,2 and 3. The nuclei were stained with DAPI (blue) and the F-actin filaments were stained with Alexa Fluor ${ }^{\circledR} 594$ Phalloidin (red). The images were overlaid with the phase contrast image to provide additional information about the scaffold morphology. The overlapping of the blue and red and dispersion by the fibers causes some of the cells to be seen pink in color. Images captured at $10 \mathrm{X}$ magnification. 

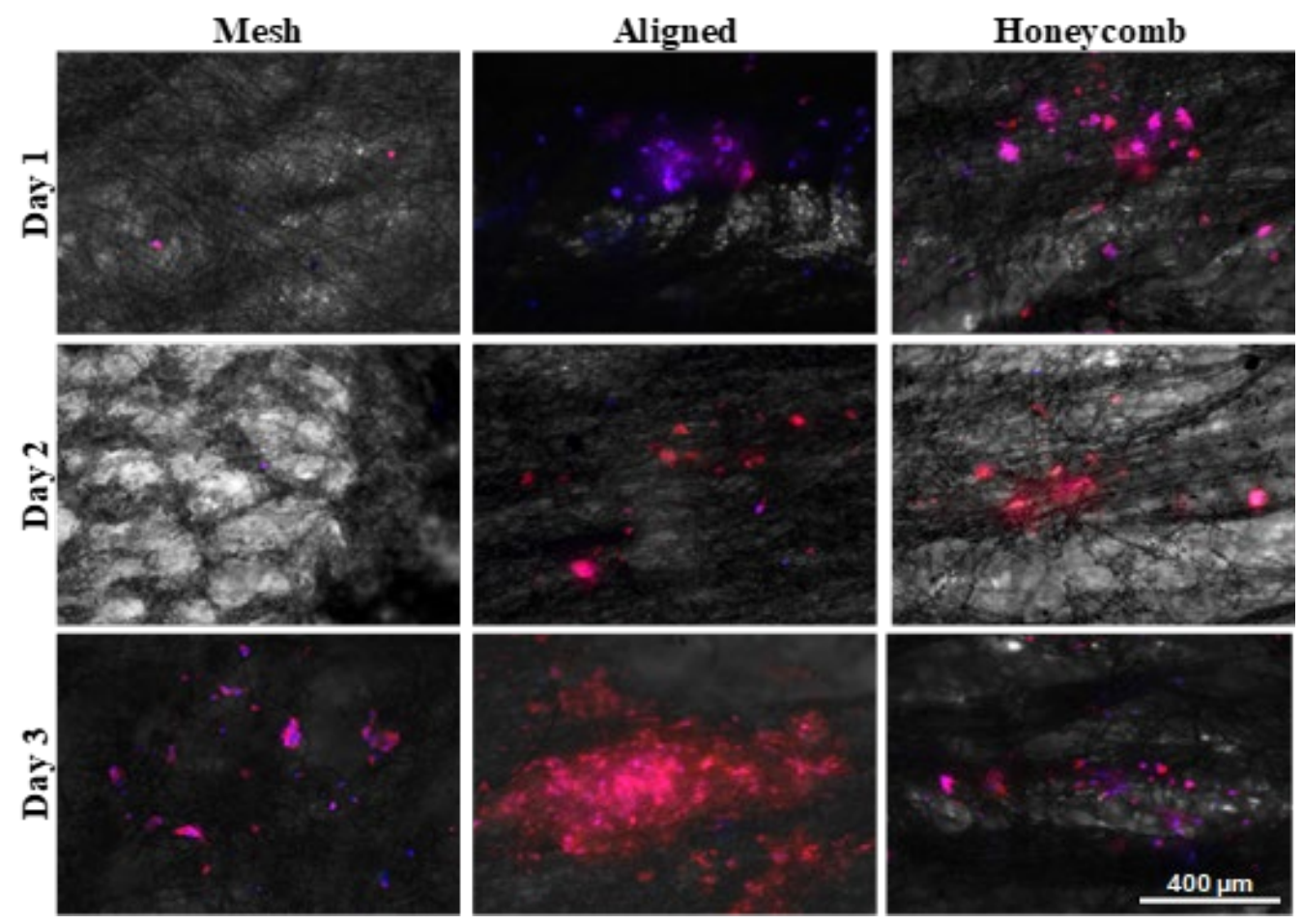

Fig. S5. Fluorescent microscope images of premalignant breast cancer cells (MCF10AneoT) on different morphologies of the PCL scaffold on days 1, 2 and 3. The nuclei were stained with DAPI (blue) and the F-actin filaments were stained with Alexa Fluor ${ }^{\circledR} 594$ Phalloidin (red). The images were overlaid with the phase contrast image to provide additional information about the scaffold morphology. The overlapping of the blue and red and dispersion by the fibers causes some of the cells to be seen pink in color. Images captured at $10 \mathrm{X}$ magnification.

\subsection{Acknowledgment}

The authors would like to acknowledge the financial support provided by the Portage Health Foundation Research Excellence Fund Research Seed Grant (PHF-REF-RS). S.N.H was supported by T3N award from the Michigan Economic Development Corporation and the PHF graduate assistantship. C. A. Q. was supported by PHF-REF-RS. B. J. V was supported by Undergraduate Research Internship Program (URIP) and Summer Undergraduate Research Fellowship (SURF) at Michigan Tech. The authors would like to acknowledge Paul Fraley, Department of Materials Science and Engineering and Dr. Kathryn Perrine, Department of Chemistry for help with mechanical characterization of scaffolds and surface characterization and Infrared Spectroscopy respectively. 


\title{
5 Scaffold morphology affects proliferation of renal, lung and bladder cancer cells
}

\author{
Samerender Nagam Hanumantharao, Carolynn Que and Smitha Rao* \\ Department of Biomedical Engineering, Michigan Technological University, Houghton, \\ MI 49931, USA \\ *Correspondence: smithar@mtu.edu
}

\begin{abstract}
Biomimetic scaffolds provide a reductionist approach to investigate the role of biophysical cues on cancer cells. In this study, we used electrospun polycaprolactone (PCL) based scaffolds of different morphologies (topography and mechanical properties), but similar surface chemistry. The scaffold morphologies were fabricated to represent the complex morphologies naturally present in the body. While the porous scaffold (honeycomb) had high mechanical strength, the interconnected network of fibers (mesh) had the lowest mechanical strength. The aligned scaffold had properties ranging between the two. Renal, bladder and lung carcinoma cell lines were seeded on the scaffolds along with a human epithelial cell line as a control. The cell-cell and cell-scaffold interactions were qualitatively analyzed along with cell motility. The proliferation of the cells on the scaffolds was quantitatively characterized. The highly metastatic and differentiated cancer cells favored the stiffer matrix while the cell lines expressing integrin $\alpha 5$ favored the mesh like architecture and low mechanical strength properties. The change in cell morphology and cell motility differed across scaffold morphologies for the same cell line. This study establishes the scaffolds as a tool to analyze the material interactions affecting cell proliferation, formation of clusters as well as migration and finds applications in understanding behavior of malignant and metastatic cancer cells.
\end{abstract}

Keywords: Electrospinning; Bladder cancer; 3D scaffolds, Tissue scaffold engineering, Renal cancer, Adenocarcinoma, Tumor modelling

\subsection{Introduction}

Biophysical cues provided by the microenvironment are crucial signals that have significant spatiotemporal effects on cell migration, differentiation, metastases and apoptosis $[295,296]$. These biophysical cues include matrix stiffness, viscoelasticity, geometrical cues, size of pores, fiber density and topography. This reciprocal interdependence between the cell and the matrix is evident in the dynamically remodeling tumor stroma, influencing complex processes such as cell adhesion, transmigration and invasion [290, 297, 298]. This remodeling leads to noticeable differences which are of clinical significance, for example, increased stiffness in the later stages of carcinoma and changes in collagen fiber morphology [264, 299-302]. There have been tremendous 
advancements on the development of physiologically relevant in vitro models to understand the role of biophysical cues. The variations in physical properties of the tumor stroma is of clinical significance and can be used for investigations into biophysical therapeutics [303]. However, majority of the research has focused on experiments on collagen-based matrices such as Matrigel ${ }^{\circledR}$, stimuli responsive polymer-based platforms, microfluidic devices and hydrogels [242, 304-307]. The complexity of the chemical composition of Matrigel along with inconsistencies in experimental results has led to a wide array of outcomes [308]. The stimuli responsive polymer-based platforms on the other hand, fail to recreate the complex architecture and anisotropy of the native tissue. While hydrogels provide different stiffnesses cells on the hydrogel respond to altered surface chemistries. Hydrogel encapsulated systems overcome some of these challenges but introduce complexities in imaging. More recently, three-dimensional (3D) substrates and microfluidic devices coupled with MEMS technology obtained using photolithography and molecular self-assembly have been reported [309-311]. The approaches used in these technologies modify the formation of focal adhesions and do not recapitulate the migration patterns seen in vivo $[312,313]$. Electrospun scaffolds provide the necessary anisotropy to replicate the 3D physical environment of the native tissue. However, lack of repeatability, use of dissimilar materials to obtain various tissue-like structures and control over the resulting structures has deterred significant research using electrospun scaffold. To overcome these challenges, we have previously used a self-assembly based electrospinning method to generate 3D structures with complex topographies [78]. In this study, we used 3D scaffolds with controlled geometries and stiffness with similar surface properties for understanding the mechanical properties of the microenvironment that interact with established cancer cell lines. A reductionist approach was used to focus on the topography and stiffness as two parameters of interest while there were no changes in the biochemical cues provided by the scaffolds [314]. Model renal, and bladder cancer cell lines and a lung carcinoma cell line were selected based on their origin and pathology [315-317]. The cells were seeded on the different morphologies of the scaffold and cultured under identical conditions. The differential interactions of the carcinoma cell lines with the scaffold demonstrated a method of in vitro tumor modelling, tumor engineering and exhibited potential for scaffold-based therapies.

\subsection{Materials and Methods}

\subsubsection{Fabrication and characterization of scaffolds}

A sol-gel of 20\% Polycaprolactone (PCL: Mw 70,000 GPC; Scientific Polymer Products, USA) in chloroform (Sigma Aldrich, USA) was used for electrospinning with parameters and conditions as described in our previous work [314]. In brief, the voltage, rotational speed of the rotating collector and the polymer feed rate were varied between $10 \mathrm{kV}$ to $11 \mathrm{kV}$, 150-300rpm and 3-4 $\mu 1 / \mathrm{min}$, respectively. Polycaprolactone (PCL) scaffolds of three different morphologies- aligned, honeycomb and mesh were obtained by manipulating the electrospinning parameters. The scaffolds were visualized using field emission scanning 
electron microscope (FESEM; Hitachi S-4700 FE-SEM) after sputter coating with an $\mathrm{Au} / \mathrm{Pd}$ target. The alignment of fibers was characterized from the FESEM image [318].

\subsubsection{Cell culture}

Clear cell renal carcinoma (Caki-1/ATCC HTB-46 ${ }^{\mathrm{TM}}$ ), renal cell adenocarcinoma (786-O /ATCC ${ }^{\circledR}$ CRL-1932 ${ }^{\mathrm{TM}}$ and ACHN/ATCC CRL-1611 ${ }^{\mathrm{TM}}$ ), bladder grade IV transitional cell carcinoma (TCCSUP/ATCC ${ }^{\circledR} \mathrm{HTB}-5^{\mathrm{TM}}$ ), bladder transitional cell papilloma (RT4/ATCC® HTB-2 ${ }^{\mathrm{TM}}$ ), bladder transitional cell carcinoma (T24/ATCC® HTB-4 ${ }^{\mathrm{TM}}$ ), lung carcinoma (A549/ATCC ${ }^{\circledR}$ CCL-185 ${ }^{\mathrm{TM}}$ ) and human embryonic kidney epithelial cells (HEK-293/ATCC® CRL-1573 ${ }^{\mathrm{TM}}$ ) were purchased from American Type Cell Culture (ATCC). The cell lines were cultured in the complete growth medium and standard culture conditions (Temperature: $37^{\circ} \mathrm{C}$, Relative humidity: $65 \%$ and $5 \% \mathrm{CO} 2$ ) as prescribed by the manufacturer. The cells in viable passages were trypsinized in the log phase and 1500 cells were seeded on the scaffolds $\left(0.25 \mathrm{~cm}^{2}\right.$ : cell viability studies and $0.5 \mathrm{~cm}^{2}$ : fluorescent staining).

\subsubsection{Cell proliferation}

The reduction of resazurin (CellTiter-Blue ${ }^{\circledR}$ Cell Viability Assay (Promega, Madison, WI)) was used to quantify the cell proliferation and metabolic activity on days 1,2 and 3 after cell seeding $(n=9)$. Appropriate controls were used including a positive control where the cells were cultured on tissue culture treated plates in similar conditions $(n=6)$. The fluorescence intensity of intracellular resorufin was measured in opaque walled clear bottom assay plates (Beckman Coulter DTX 880 Multimode Detector at $560 \mathrm{~nm}$ excitation and $590 \mathrm{~nm}$ emission).

\subsubsection{Fluorescent staining and analysis}

The cells seeded on the scaffolds were fixed on days 1, 2 and 3 after cell seeding using 4\% paraformaldehyde, permeabilized with Triton-X 100 and stained with DAPI (4',6diamidino-2-phenylindole) (Life Technologies, USA), Alexa Fluor ${ }^{\circledR} 594$ Phalloidin (Invitrogen, USA) in 786-O, ACHN, Caki-1, HEK-293 and A549 cells, and Alexa Fluor ${ }^{\circledR}$ 488 Phalloidin (Invitrogen, USA) in RT4, T24 and TCCSUP cells according to the manufacturer's protocols. The DAPI is a nuclear counterstain that fluoresces upon binding with A-T rich regions of dsDNA while phalloidin is a fluorescent probe that selectively stains cytoskeletal F-actin filaments. Panoramic images of the cells on scaffolds were stitched to provide a clear representation of cell-scaffold behavior. The quantitative analysis of the alignment of cells on the scaffolds were characterized using the Directionality plug-in tool in Fiji [318]. The orientation of cells seeded on different morphologies of the scaffolds counterstained with DAPI was calculated from the thresholded images (10X magnification) of cells after watershed separation. The coefficient of alignment is the highest value of alignment obtained along a specific orientation and the dispersion is the standard deviation of the gaussian function used to fit the distribution of cells on the scaffolds. A comparison of 
the coefficient of alignment of all the cell lines investigated on all three days and morphologies is represented in the supplementary information.

\subsubsection{Statistical Analysis}

The statistical evaluation of the cell proliferation was done using OriginPro $2018 \mathrm{~b}$ and IBM ${ }^{\circledR}$ SPSS statistics V25 and represented as mean \pm SEM. The orientation of the fibers is represented as a histogram plotted between the coefficient of alignment and direction between $90^{\circ}$ to $-90^{\circ}$. The orientation of the cells on the fibers is represented as coefficient of alignment and the error bars represent standard deviation. The statistical differences between different days for a particular cell line in a given morphology was calculated using One-way ANOVA followed by post-hoc Tukey's test to calculate significance at $p<0.05$ between different days for each morphology of the scaffold. The same method was used to calculate the significance between different cell lines for each of the scaffold morphologies.

\subsection{Results}

\subsubsection{Scaffold characterization}
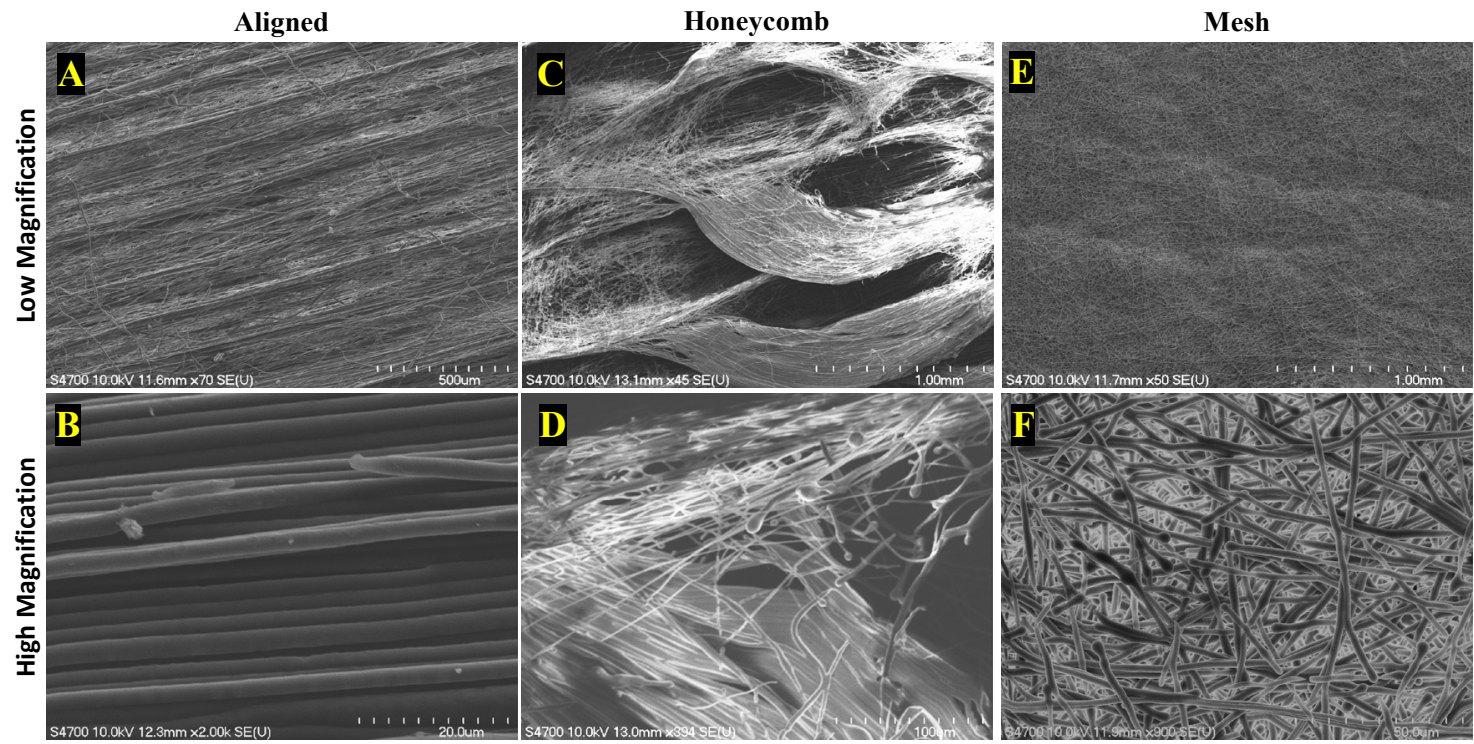

Figure 1. Field emission scanning electron microscopic (FESEM) images of PCL scaffolds exhibiting different morphologies. The low magnification images (A,C,E) are presented on top while the high magnification images $(B, D, F)$ are presented in the bottom.

A method of electrospinning with careful control of the environmental conditions enabled the repeatable fabrication of scaffolds with different morphologies. From the FESEM images, the topographies including the differential orientation of the fibers can be inferred (Figure S1). The mesh scaffolds (Figure 1A and Figure 1B) form a dense network of 
interconnected fibers of similar diameters. The internal connective spaces serve as supportive matrices and increase the porosity of the 3D structures. The aligned scaffolds (Figure 1C) had fibers of varying diameters forming bands on an underlying layer of aligned fibers with low anisotropy. The high magnification images of the aligned scaffolds (Figure 1D) revealed a dense packing of aligned fibers with similar diameters. The honeycomb scaffolds (Figure 1E) have a closely-knit honeycomb pattern with distinct porous regions underneath an array of aligned fibers. The 3D nature of the honeycomb scaffold can be easily inferred from the high magnification image (Figure 1F) which shows the walls of the honeycomb structure. The mechanical and surface characterization of the scaffolds were discussed elaborately in our previous study [314]. A tight control over the electrospinning parameters and good manufacturing practices ensured repeatability of scaffold morphology and mechanical properties.

\subsubsection{Fluorescence microscopy}

Cancer cell lines representing distinct stages of renal, bladder and lung cancer were used to evaluate the cell-scaffold interactions. The cells were counterstained for cytoskeleton (F-Actin) and nucleus (DAPI).

\subsubsection{786-O}

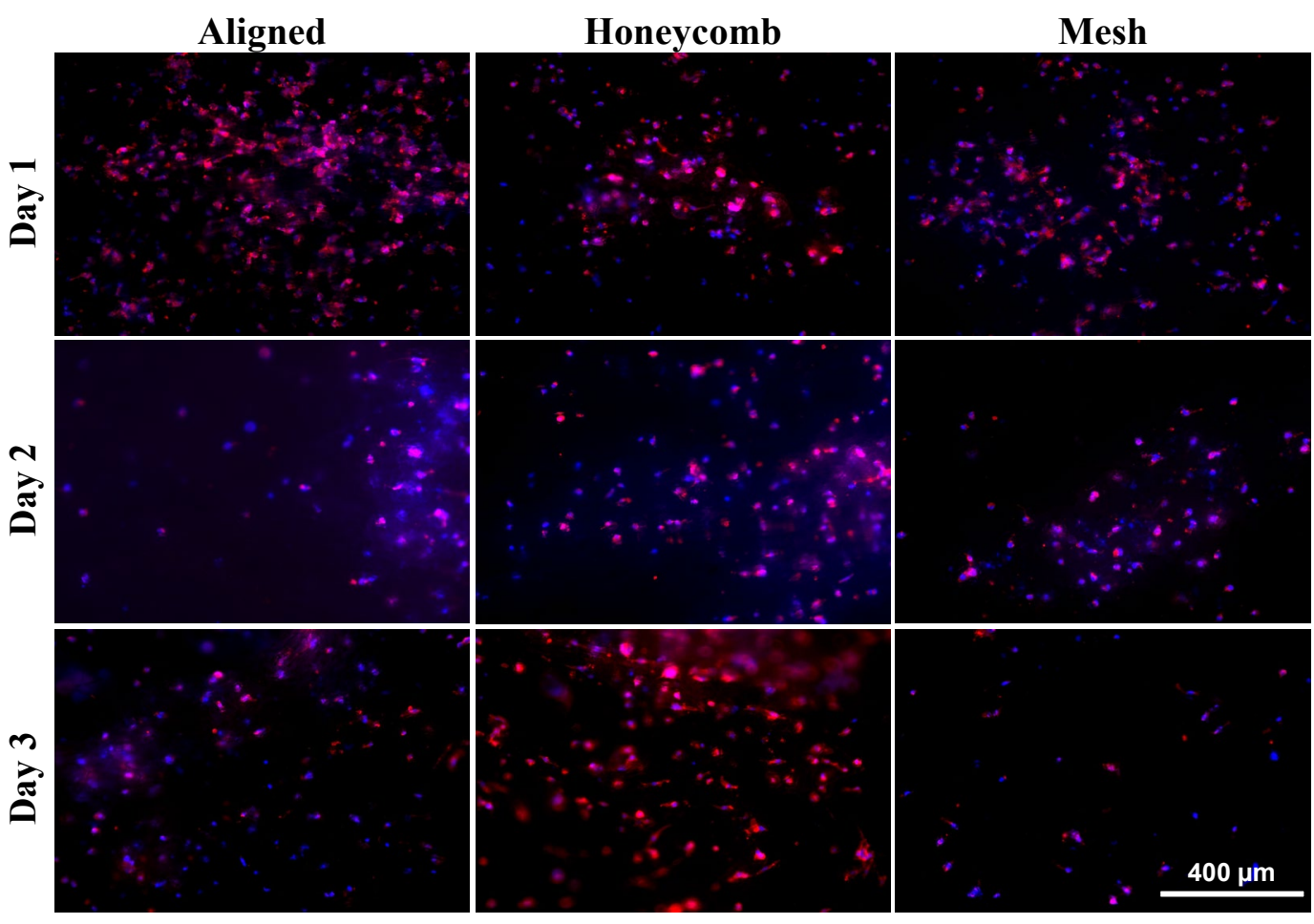

Figure 2. Fluorescent microscopy images of renal adenocarcinoma cells (786-O) on PCL scaffolds of different morphologies on days 1,2 and 3. The nuclei were stained with DAPI 
(blue) and the actin filaments were stained with Alexa Fluor ${ }^{\circledR} 594$ Phalloidin (red). All images captured at $10 \mathrm{X}$ magnification.

Qualitative characterization of the interaction of 786-O cells with different morphologies of the 3D scaffold revealed differences in coefficient of alignment (Figure S2) and cell behavior within each morphology (Figure 2). The cells had a distinguishing difference in behavior after 24 hours of cell seeding. The cells were clumped in groups but had an underlying orientation on the aligned scaffolds. The cells on honeycomb scaffolds were clumped in regions with some cell infiltration as inferred from the out-of-focus regions. The cells on all three morphologies of the scaffolds after 48 hours had reduced clumping, than the first 24 hours. The cells had relatively better infiltration on the aligned and honeycomb scaffolds compared to cells on the mesh scaffold. This was more evident on day 3 on the honeycomb scaffolds with cells having a fibroblast like morphology traversing across the different layers of the honeycomb scaffolds. The cells on the aligned scaffold had reduced cell spreading and increased cell clustering compared to day 2 . The cells on day 3 in the mesh scaffolds had increased cell motility which enabled their spreading along the scaffold (Figure S3). Based on the difference in cell distribution and morphology, it appears there is a preference of the cells based on biophysical cues towards the mesh scaffolds.

\subsubsection{ACHN}

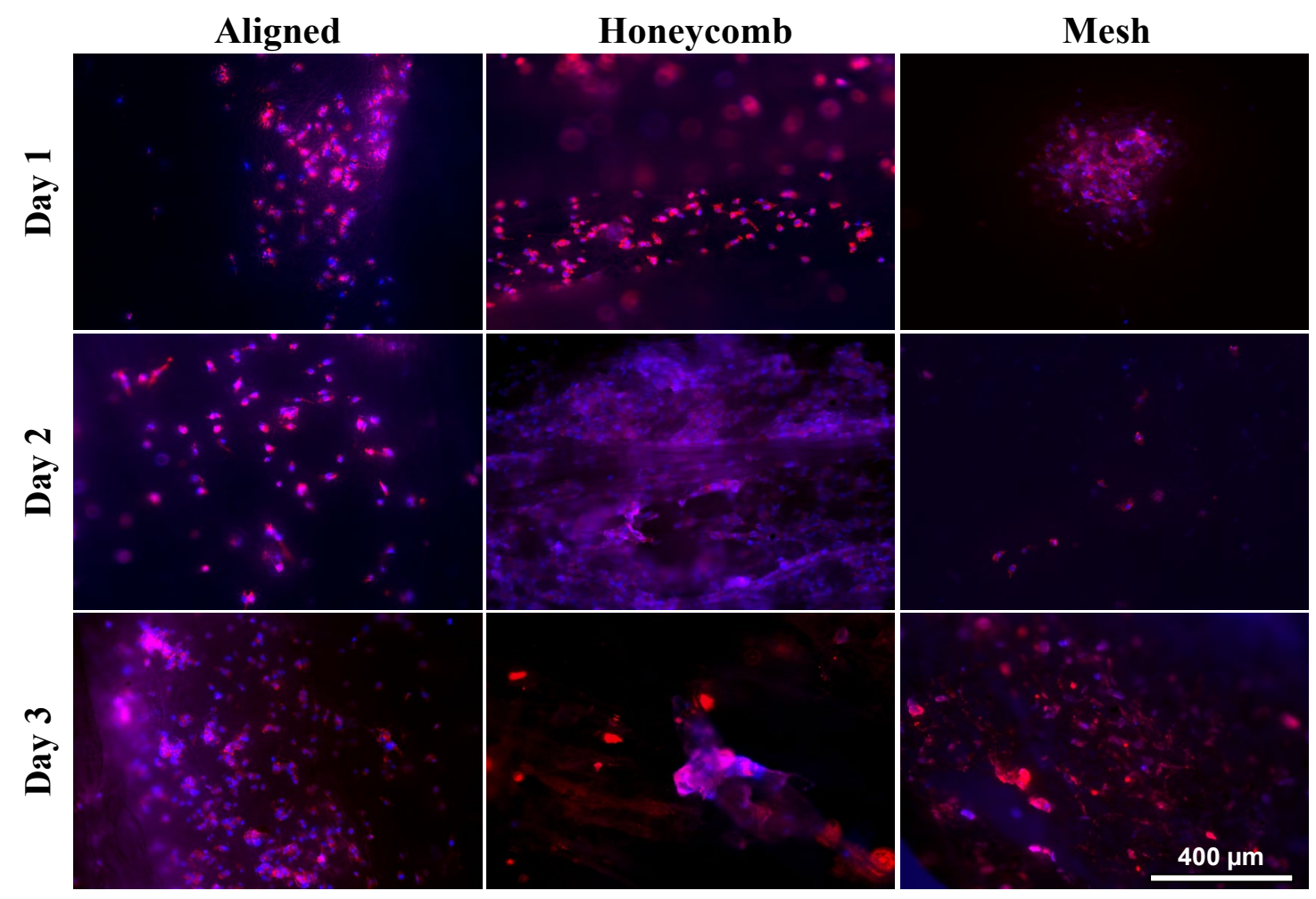


Figure 3. Fluorescent microscopy images of renal adenocarcinoma cells (ACHN) on PCL scaffolds of different morphologies on days 1,2 and 3. The nuclei were stained with DAPI (blue) and the actin filaments were stained with Alexa Fluor ${ }^{\circledR} 594$ Phalloidin (red). All images captured at 10X magnification.

The behavior of ACHN cells seeded on the three different morphologies of scaffolds were analyzed (Figure 2 and Figure S2). The cells penetrated easily through the depths of the scaffold within the first 24 hours. This is evident in the scaffolds with the aligned and honeycomb morphology. The cells on aligned morphology on day 2 had some spreading of cells with decreased clumping across the scaffolds. The cells on honeycomb morphology, however, did not spread across the scaffolds but accumulated in certain regions of the scaffold. The cells on mesh morphology on day 2 were distributed throughout the scaffold and there were very few regions where cells had clumped. Panoramic images (Figure S4) of the scaffolds of all three morphologies on day 2 provide a better representation of the differential interactions of the cells with the scaffolds. On day 3 , the cells on the aligned scaffolds migrated further with some clumping of cells. The cells on the honeycomb scaffolds did not migrate, with a high concentration of cells in a small region of the scaffold. The cells within the mesh morphology migrated and were well distributed. A comparison of the panoramic images (Figure S5) between the scaffolds with aligned and mesh morphology illustrated the role of topographical and durotactic cues in cell behavior qualitatively. The cells on the aligned scaffold were oriented along the alignment of the scaffold and migrated along with the contact guidance provided by the aligned fibers. The cells on the mesh scaffolds were spread across with less clumping. The differences in cell morphology for the renal adenocarcinoma cell line is well defined when comparing between the different scaffolds. 


\subsubsection{Caki-1}

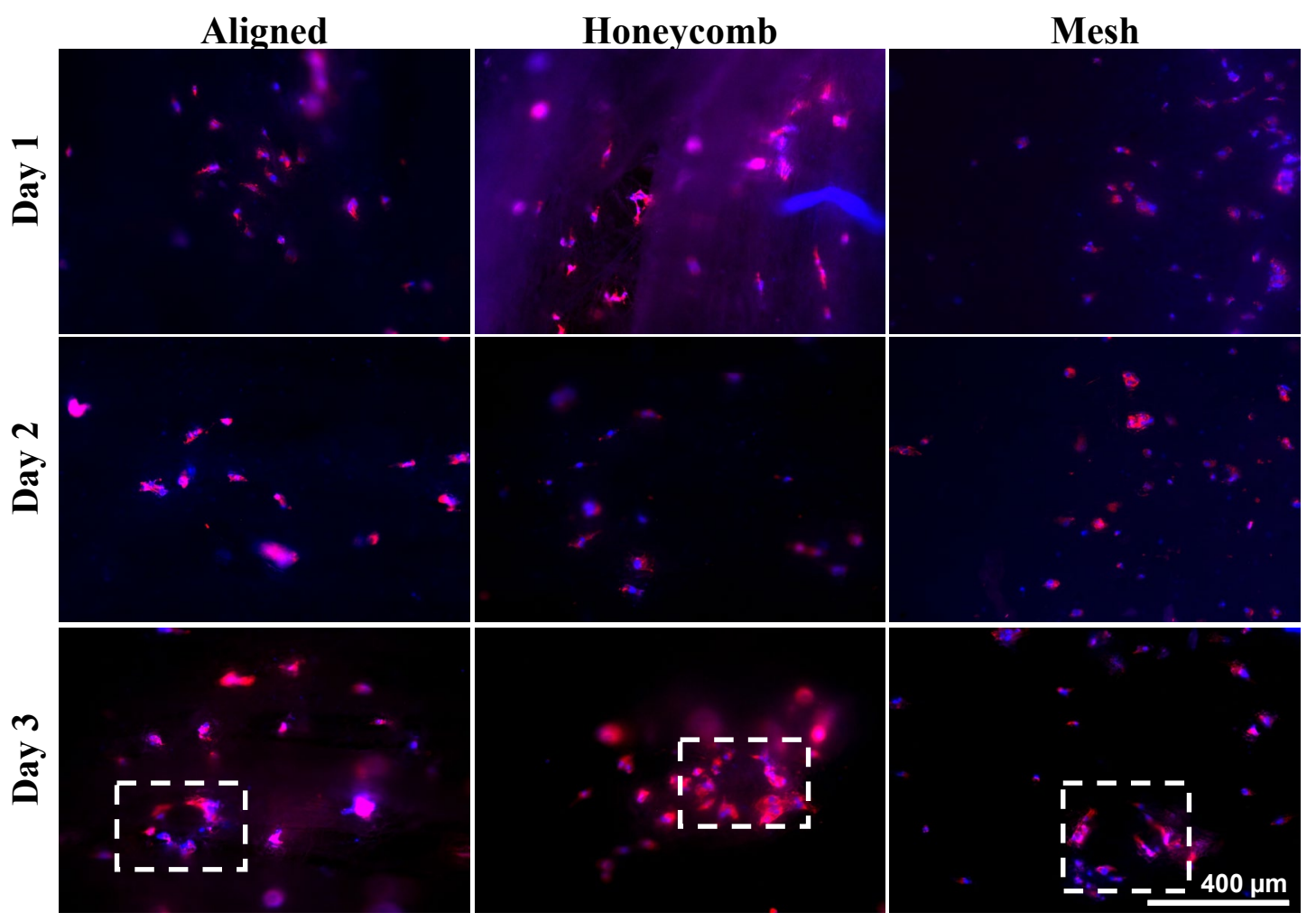

Figure 4. Fluorescent microscopy images of clear cell renal carcinoma cells (Caki-1) on PCL scaffolds of different morphologies on days 1, 2 and 3. The nuclei were stained with DAPI (blue) and the actin filaments were stained with Alexa Fluor ${ }^{\circledR} 594$ Phalloidin (red). All images captured at 10X magnification. The white dashed box shows the formation of complex structures similar to a convoluted tubule.

Qualitative analysis of the behavior of Caki-1, a model line of metastatic clear cell (cc) renal cell carcinoma (RCC) with scaffolds was done by staining the nucleus and F-actin filaments (Figure 4 and Figure S2). The cells had little or no alignment on day 1 across all the morphologies of the scaffold. However, the cells infiltrated through the different layers of the scaffold even on day 1 , with the infiltration being most evident for the honeycomb morphology. The cells were more spread out on day 2 on all the morphologies, with cellular infiltration and migration being more pronounced. The cells infiltrated and migrated better on the mesh scaffolds. The cells were oriented along the direction of fibers on day 3 in the aligned scaffolds. The cells were found in localized regions (inside pores) in the honeycomb morphology. The cells were spread out relatively more on the mesh scaffolds than the other scaffold morphologies. Interestingly, the polygonal shaped cells on the three morphologies of the scaffolds formed complex structures resembling tubules on day 3 as highlighted by the rectangular boxes in figure 4 . Although there were no clear distinctions about the topographical preferences between the three scaffolds, the $3 \mathrm{D}$ fibrous nature of the scaffolds helped in forming tubules without external growth factors. 


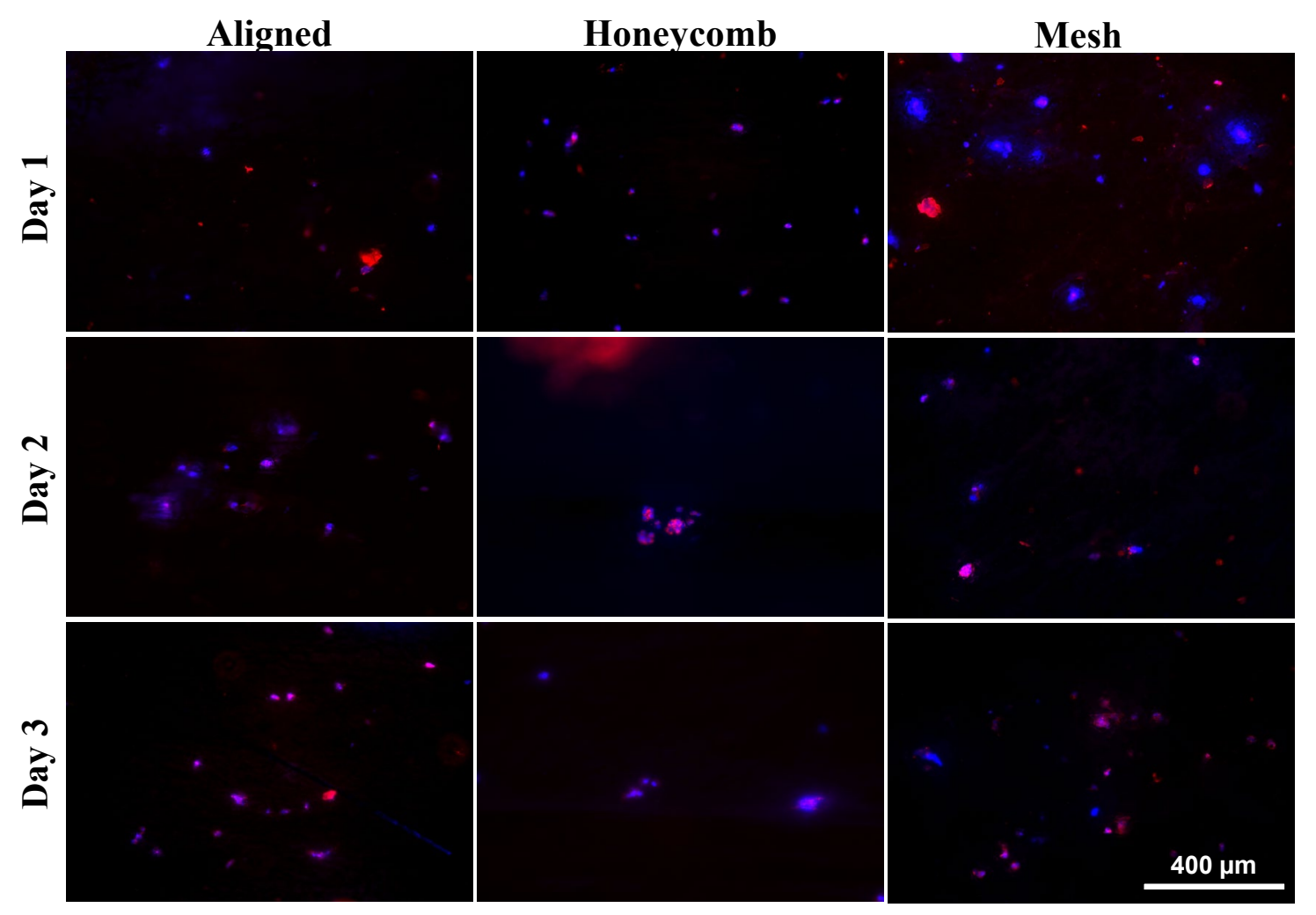

Figure 5. Fluorescent microscopy images of human embryonic kidney cells (HEK-293) of epithelial morphology on PCL scaffolds of different morphologies on days 1,2 and 3. The nuclei were stained with DAPI (blue) and the actin filaments were stained with Alexa Fluor ${ }^{\circledR} 594$ Phalloidin (red). All images captured at 10X magnification.

The human embryonic kidney epithelial cells were studied as a non-cancerous cell line. The morphology of the cells on the scaffolds were analyzed to provide qualitative results on scaffold-cell interactions including adhesion, spreading and coefficient of alignment (Figure 5 and Figure S2). The cells were isolated on day 1 in the aligned scaffolds compared to the mesh scaffolds where groups of cells or colonies were formed. The cells on the honeycomb scaffolds were spread out on day 1 but formed colonies with groups on day 2. The cells infiltrated through the different layers of the aligned scaffold on day 2 with spreading of the cells across the scaffold. There was little or no alignment of cells in the mesh morphology on day 2 and 3. The cells infiltrated through the different layers of the honeycomb scaffold on day 3. The panoramic image (Figure S6) provides a better representation of the non-preferential orientation and spreading of cells on the aligned and mesh morphology. 


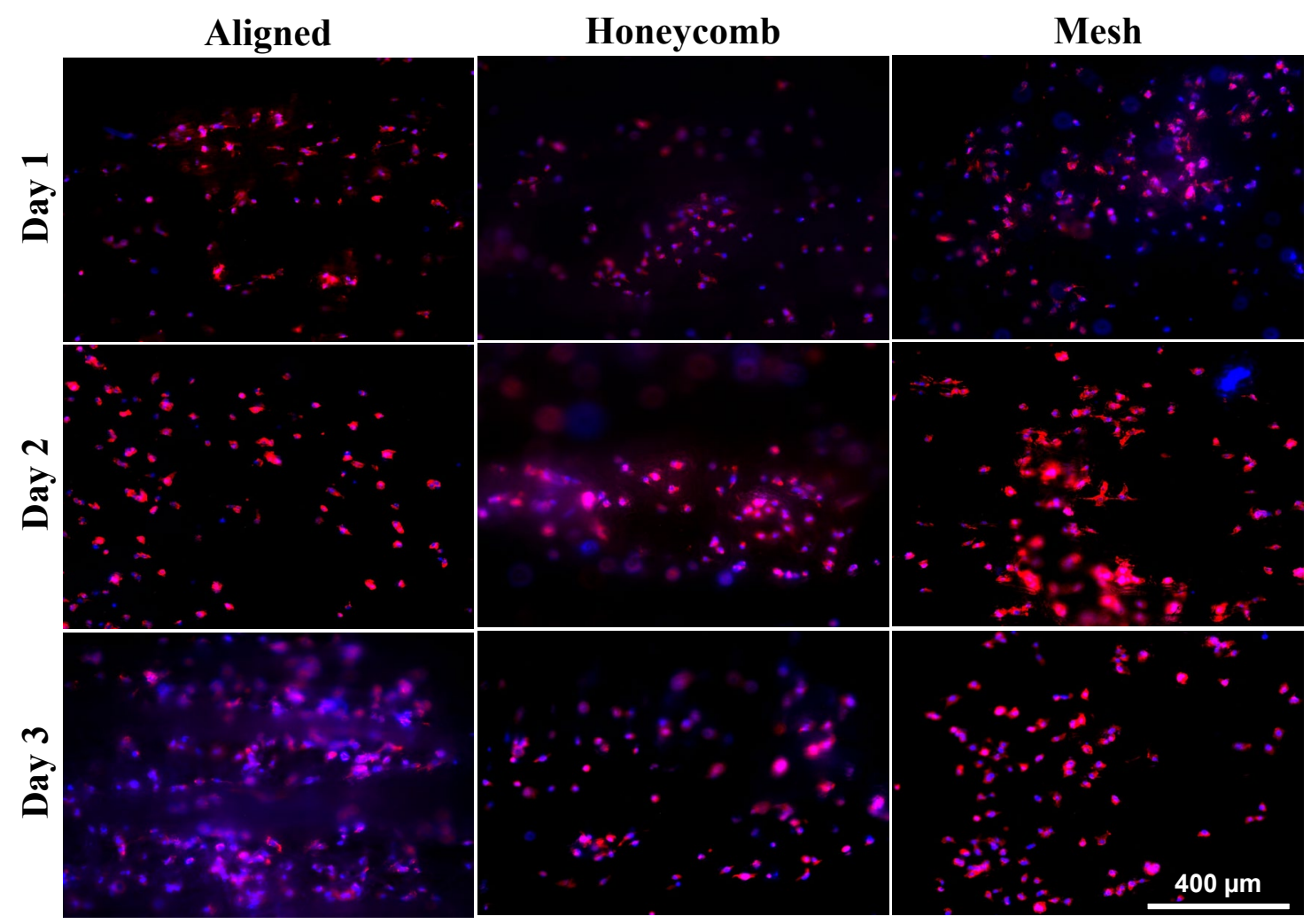

Figure 6. Fluorescent microscopy images of lung carcinoma cells (A549) on PCL scaffolds of different morphologies on days 1,2 and 3. The nuclei were stained with DAPI (blue) and the actin filaments were stained with Alexa Fluor ${ }^{\circledR} 594$ Phalloidin (red). All images captured at 10X magnification

The qualitative analysis of lung carcinoma cell line (A549) seeded on the scaffolds was inferred from figure 6. The coefficient of alignment of the cells on the scaffolds is represented in figure $\mathrm{S} 2$. The cells had little to no clumping on all the morphologies on day 1. The cells responded to the cues provided by the honeycomb scaffolds by aligning along the walls of the honeycomb structure. The cells infiltrated across the different layers of the scaffold in the honeycomb scaffold on day 2 and were uniformly distributed after 48 hours. The cells were elongated with a fibroblast like morphology on day 3 in all the scaffolds. The infiltration and selective localization of cells in the aligned scaffolds can be deduced from the image on day 3 . This localization is more pronounced in the honeycomb scaffolds as inferred from figure S7. The cells did not spread across the different regions of the scaffold but were localized to certain regions of the honeycomb scaffold. The cues provided by the scaffolds helped in influencing the cell morphology and motility. 

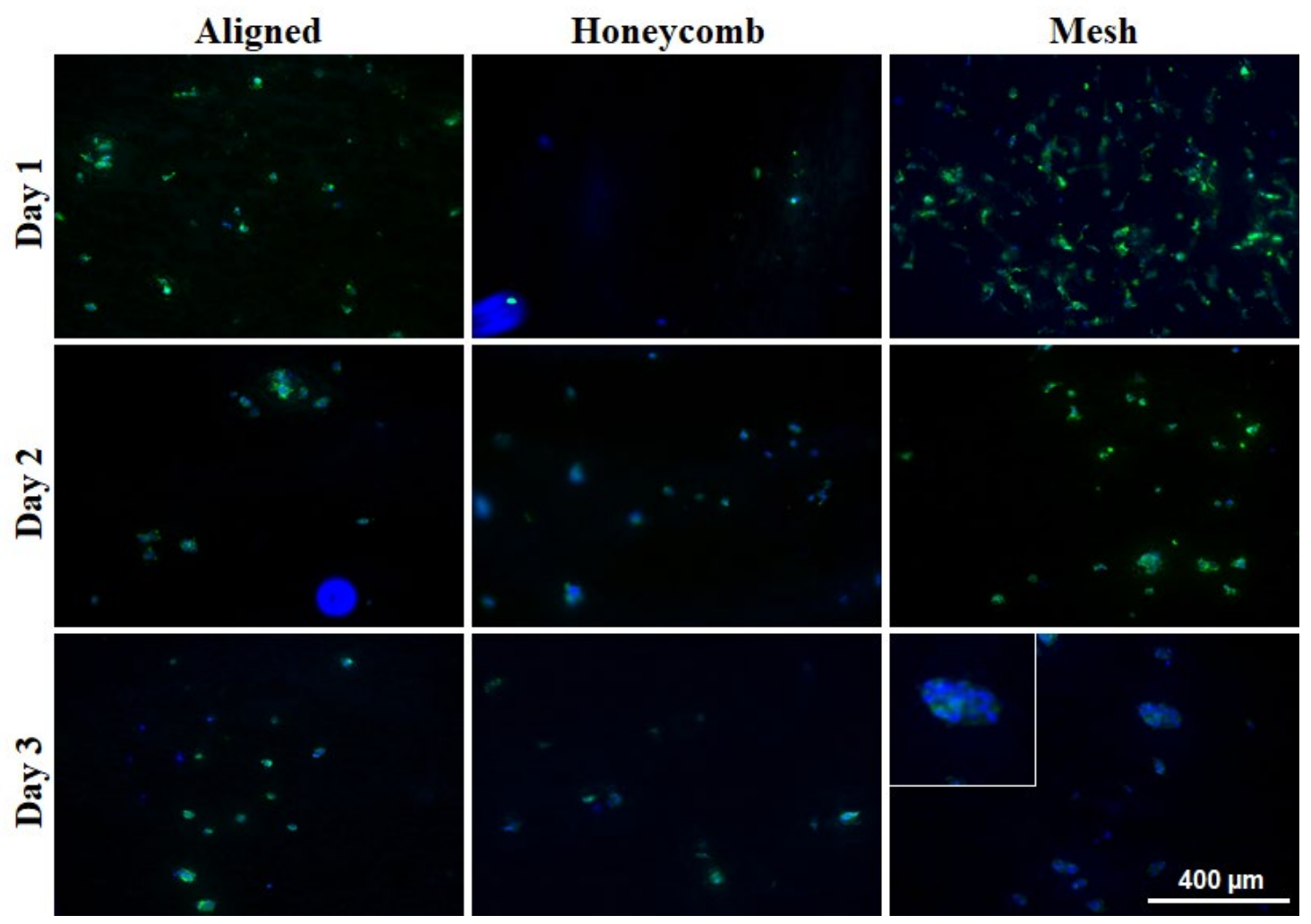

Figure 7. Fluorescent microscopy images of bladder transitional cell papilloma cells (RT4) on PCL scaffolds of different morphologies on days 1,2 and 3. The nuclei were stained with DAPI (blue) and the actin filaments were stained with Alexa Fluor ${ }^{\circledR} 488$ Phalloidin (green). The inset shows an enlarged image of the regions of interest. All images were captured at $10 \mathrm{X}$ magnification.

The morphology of the bladder transitional cell papilloma cells (RT4) on different scaffold types were qualitatively analyzed using fluorescence microscopy (Figure 7 and Figure S2). The cells were spread out more uniformly on the aligned and honeycomb scaffolds on day 1. The cells were elongated with a fibroblast like morphology on day 1 in the mesh scaffolds. The cells infiltrated through the depths of the scaffolds and were spread apart on day 2 and day 3 in the aligned and honeycomb scaffolds. The cells were elongated in the honeycomb scaffolds on day 3 while the cells were clumped together resembling papilloma in the mesh scaffolds. A panoramic image of the cells on the scaffolds on day 3 (Figure S8) shows the localization of cells in mesh scaffolds and the dissemination of cells on the aligned and honeycomb morphology. 


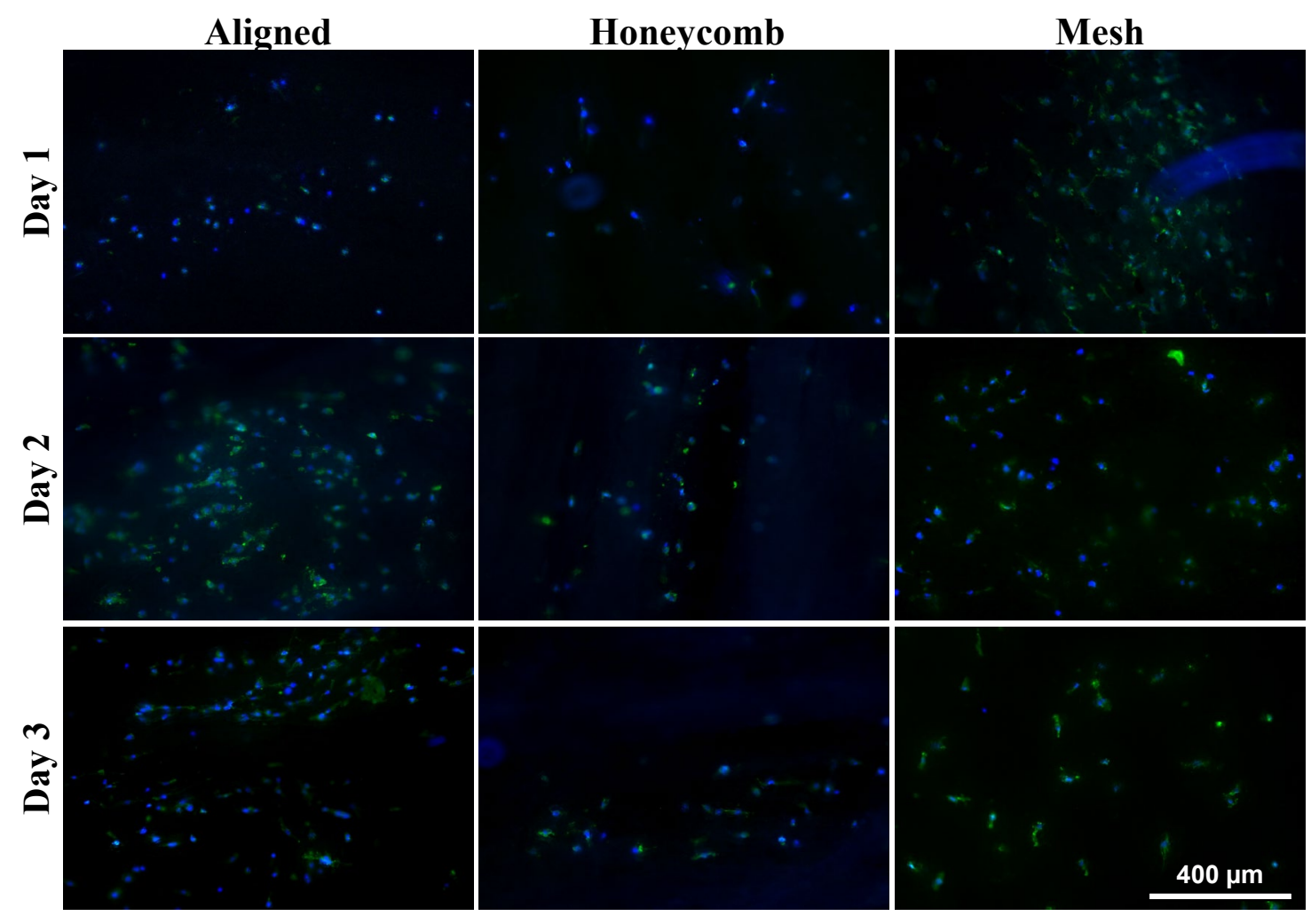

Figure 8. Fluorescent microscopy images of bladder transitional cell carcinoma cells (T24) on PCL scaffolds of different morphologies on days 1,2 and 3. The nuclei were stained with DAPI (blue) and the actin filaments were stained with Alexa Fluor ${ }^{\circledR} 488$ Phalloidin (green). All images were captured at 10X magnification.

The bladder transitional cell carcinoma cell (T24) morphology was analyzed qualitatively using fluorescence microscopy (Figure 8 and Figure S2). The cells on the aligned and honeycomb scaffolds on day 1 were spread across the scaffold with little or no clumping. The cells on mesh scaffolds were elongated and were concentrated in regions on days 1 and 2. On day 2, the cells on aligned scaffolds were elongated and oriented along the direction of fibers. The cells on the honeycomb scaffold infiltrated through the depths of the scaffold. The cells on the mesh scaffold were spread out further than day 2 with infiltration in regions. On day 3, the cells on the aligned scaffolds were elongated and oriented along the direction of the fibers with little or no clumping. The elongated cells on the aligned scaffolds were similar in morphology to the muscle invasive bladder cancer [319]. On day 3, the cells on the honeycomb scaffold were present at different layers with elongations along the direction of the fibers while the cells on the mesh scaffold were spread further apart than either of the two morphologies. The cells were present on different layers of the scaffolds as inferred from the different planes of focus. The topotactic and 
durotactic behavior of cells were observed with distinct changes in cellular morphology and infiltration pattern.

\subsubsection{TCCSUP}

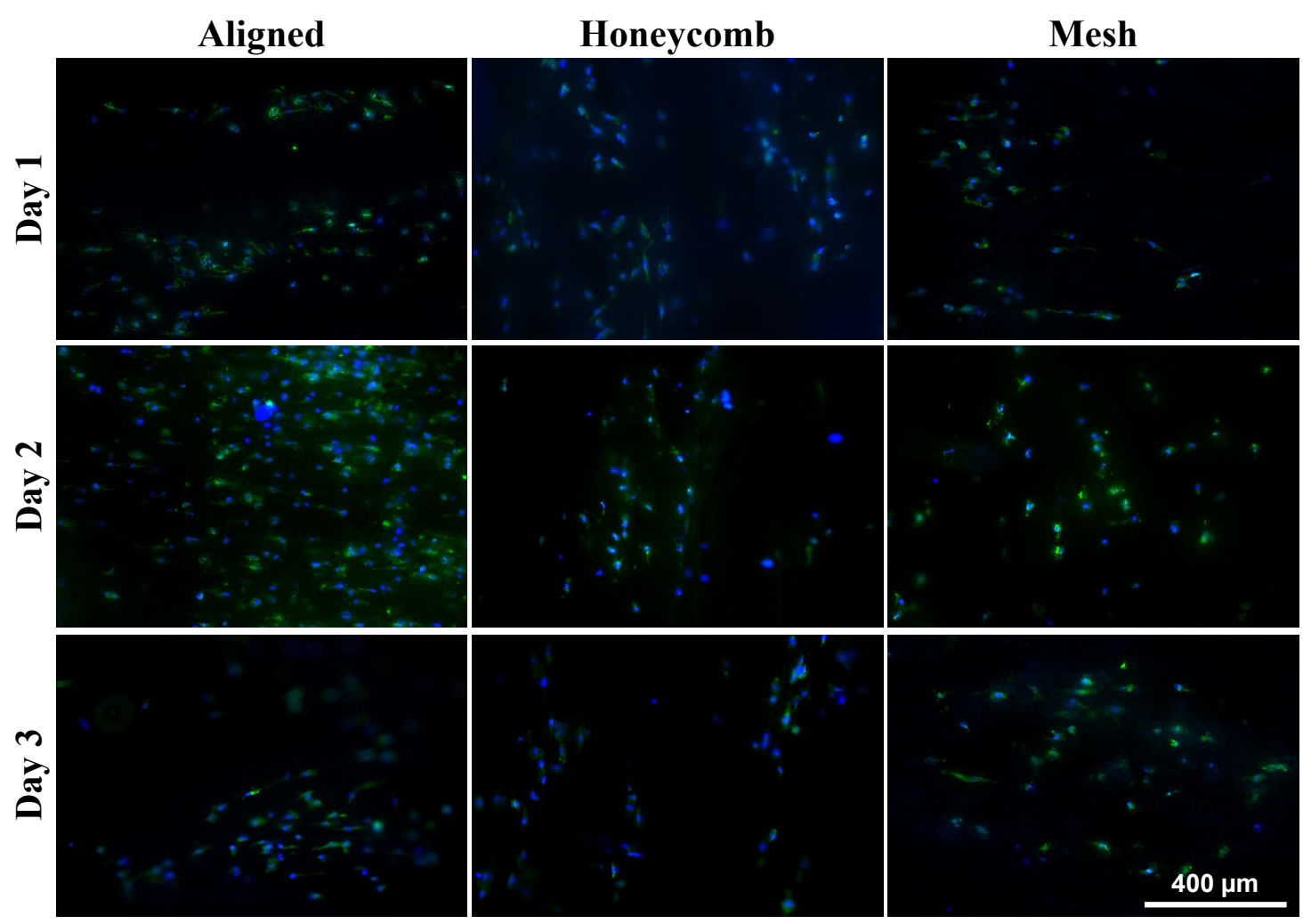

Figure 9. Fluorescent microscopy images of bladder grade IV transitional cell carcinoma (TCCSUP) on PCL scaffolds of different morphologies on days 1, 2 and 3. The nuclei were stained with DAPI (blue) and the actin filaments were stained with Alexa Fluor ${ }^{\circledR} 488$ Phalloidin (green). All images were captured at 10X magnification.

The morphology of the bladder grade IV transitional cell carcinoma (TCCSUP) on different scaffold types were qualitatively analyzed using fluorescence microscopy (Figure 9 and Figure S2). On day 1, the cells were distributed uniformly on the aligned scaffolds and oriented along the direction of the fibers. This behavior was consistent on day 2 as well. The cells on the honeycomb scaffolds were aligned along the direction of alignment of the walls of the pores on days 1 and 2 . The cells were elongated in the mesh scaffolds on day 1 with some cell infiltration on day 2 . The elongation of the cells along the orientation of the fibers and cellular infiltration is more pronounced on day 3 in the aligned scaffolds. The cells were present along the direction of the walls of the honeycomb pores on day 3. There were little or no cells present inside the pores. The cells in the mesh scaffolds were randomly oriented with some cell infiltration. A panoramic image of the cells on the scaffold (Figure S9) helps reiterate the preferential binding of the cells after 24 
hours to larger diameter and tightly packed fibers. The larger diameter fibers have higher tensile strength than the smaller diameter fibers [320]. This preferential binding of cells to fibers demonstrates the responsiveness of cells to mechanical and spatial cues.

\subsubsection{Cell Proliferation}
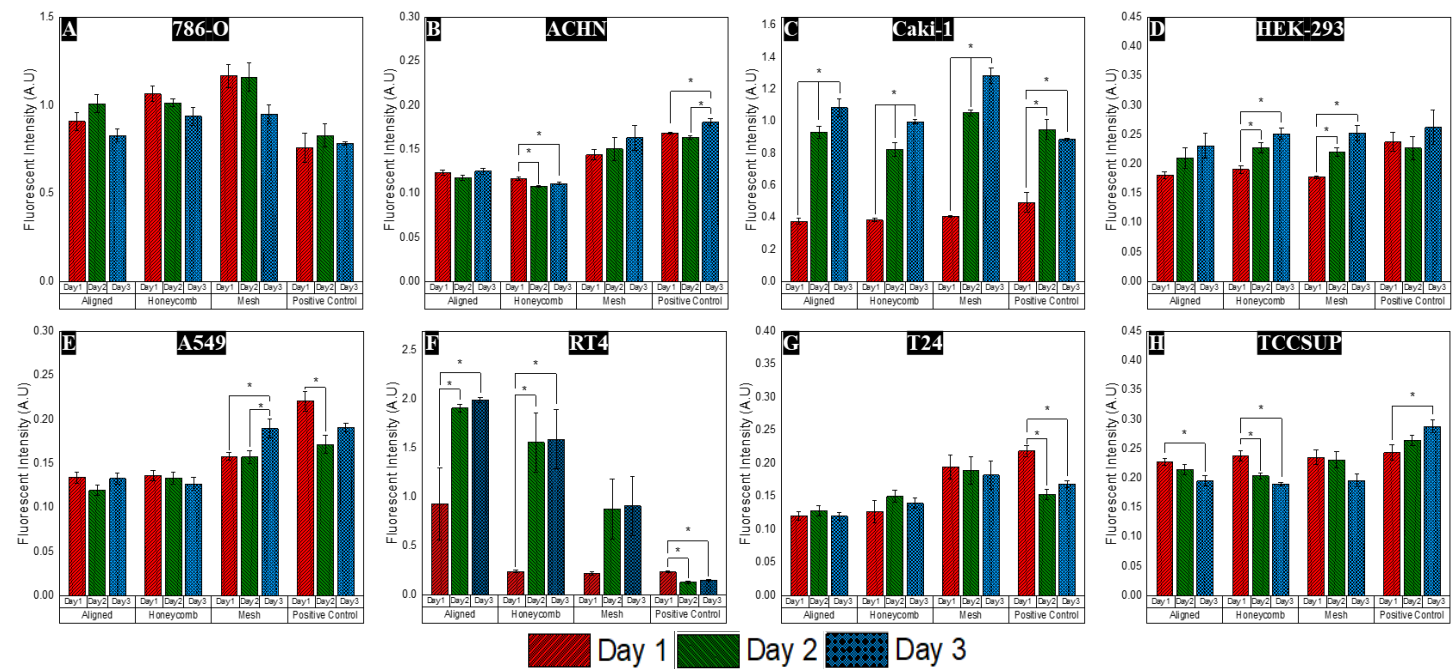

Figure 10. Quantification of cell proliferation on days 1, 2 and 3 on different scaffold morphologies and cell lines. A) The proliferation of 786-O (renal adenocarcinoma) cells on different morphologies of the scaffold. Cells seeded on scaffolds had higher cell proliferation on the scaffolds compared to the $2 \mathrm{D}$ positive control. B) The proliferation of ACHN (renal adenocarcinoma) cells on different morphologies of the scaffold. Cells seeded on the mesh scaffolds had higher proliferation and viability compared to other scaffold morphologies. C) The proliferation of Caki-1 (clear cell renal carcinoma) cells on different morphologies of the scaffold. Cells seeded on the mesh scaffolds had higher cell metabolism compared to other scaffold morphologies. D) The proliferation of HEK-293 (human embryonic kidney cells) cells on different morphologies of the scaffold. The proliferation across all different morphologies and positive control were similar. E) The proliferation of A549 (lung carcinoma) cells on different morphologies of the scaffold. Cells seeded on the mesh scaffolds and positive control had high cell proliferation and viability compared to other scaffold morphologies. F) The proliferation of RT4 (bladder transitional cell papilloma) cells on different morphologies of the scaffold. The cells preferred the 3D scaffolds compared to the $2 \mathrm{D}$ positive control. The cells preferred the scaffolds with contact guidance. G) The proliferation of T24 (bladder transitional cell carcinoma) cells on different morphologies of the scaffold. Cells seeded on the mesh scaffolds had higher cell proliferation compared to other scaffold morphologies. H) The proliferation of TCCSUP (bladder grade IV transitional cell carcinoma) cells on different morphologies of the scaffold. The cells preferred scaffolds with high mechanical strength and stiffness. Error bars are represented through the standard error of mean and statistical significance is indicated by $\mathrm{p}$ values $\left({ }^{*} \mathrm{p}<0.05\right)$. 
The reduction of resazurin to resorufin is a suitable tool to assess the cell proliferation based on the metabolic activity of cells [321]. The primary renal adenocarcinoma cell line (786-O) had a higher proliferation rate in the 3D scaffold with strong preference to mesh scaffolds compared to the 2D positive control (Figure 11A). T. renal adenocarcinoma established through pleural effusions (ACHN) demonstrated a similar trend showing a strong preference to the mesh scaffolds (Figure 11B). However, the positive control had higher cell proliferation on all three days compared to the 3D scaffolds. The increase in cell proliferation on day 3 in the positive control was statistically significant compared to the other two days. The clear cell renal carcinoma (Caki-1) had a strong preference towards the 3D scaffolds especially the mesh morphology (Figure 11C). The increase in cell proliferation was statistically significant across all days and morphologies of scaffolds. The cell proliferation in aligned and honeycomb scaffolds were comparable. The human embryonic kidney cells had similar cell proliferation irrespective of the scaffold morphology (Figure 11D). The increase in cell proliferation was statistically significant between day 1 and day 3 for the honeycomb and mesh morphologies. The lung carcinoma (A549) exhibited an entirely different trend where the cell proliferation rates decreased on the positive control between days 1 and 2 (Figure 11E). The cells preferred the mesh morphology among all the morphologies of the scaffold. The increase in cell proliferation on day 3 was statistically significant in the mesh scaffolds. The cell proliferation rates on the 3D scaffolds was incrementally higher than the proliferation rates of the bladder transitional cell papilloma (RT4) model cell line (Figure 11F). The cells preferred the aligned scaffolds among the other two morphologies with a statistically significant increase over all three days. The honeycomb scaffolds were preferred over the mesh scaffolds by the RT4 cells. The T24 cells preferred the mesh scaffolds over the aligned or honeycomb scaffolds (Figure 11G). The metastatic cell line TCCSUP favored the 2D positive control over the 3D scaffolds (Figure 11H). Among the scaffolds, the cells preferred the honeycomb scaffold. The cells had a declining trend in cell proliferation or metabolism across all three days in all the scaffolds.

\subsection{Discussion}

The cell proliferation, cell-scaffold interactions and changes in cellular morphology were evidently influenced by the geometric or topographical cues of the matrix and enhanced or antagonized by the stiffness of the scaffold matrix. Cancer cell lines representing distinct types of renal cancer seeded on the scaffolds showed a strong preference to the mesh scaffolds. The 786-O cells preferred the 3D platform which agreed well with the findings by Pan and colleagues [322]. However, the 786-O renal carcinoma cells preferred the mesh scaffolds over the honeycomb or aligned scaffolds which had the least stiffness and anisotropy. The 786-O which expresses integrin $\alpha 5$ and $\beta 1, \beta 3$ has a differential expression on day 3 with a more invasive fibroblast morphology versus an epithelial morphology on day $1[323,324]$. This mechanosensing by integrin $\alpha 5$ begins almost instantaneously as shown by Strohmeyer and colleagues [325]. The single and collective motility of metastatic cancer cells was dependent on the lower stiffness of the matrix [326, 327]. This behavior was more prominent in the case of ACHN cells seeded on the scaffolds. The lack of cell 
motility in the honeycomb fibers compared to even distribution in the mesh scaffolds which are consistent with the observations made by Kristal-Muscal et al. on the interactions of metastatic cancer cells with a compliant substrate [328]. The indentation of mechanically compliant substrates by the highly metastatic ACHN cells leads to better cell motility and spreading in the mesh scaffolds. [329] The spreading of cells is inversely proportional to the stiffness of the scaffolds. However, the positive control with the highest stiffness and 2D structure had the highest cell proliferation. We factor this to the reduced proliferation and increased differentiation of the integrins in 3D spheroids compared to 2D monolayers [330]. The localization of the cells in honeycomb structures compared to aligned distribution of fibers in the aligned scaffolds point towards an amoebic mode of migration with reduced proteolytic dependent migration [331]. We attribute this to the limits on nuclear deformation restricting migration through the pores [332]. The lack of migration and cell invasion leads to a decline in cell proliferation rates indicating porous scaffolds as a potential regulator of transmigration and invasion. This is due to Yes-associated protein (YAP) and YAP-mediated cross-regulating feedback mechanisms: YAP Wilms Tumor-1YAP-mediated downregulation leading to reduction of E-Cadherin expression and YAPTRIO-Merlin mediated regulation of Rho GTPase family proteins which increases cell motility [333]. Caki-1, a cell line to model metastatic clear cell (cc) renal cell carcinoma (RCC) has been shown to demonstrate similarities to the human proximal tubule epithelium [334]. These structures were present on all the scaffolds on day 3 . The cell proliferation behavior of Caki-1 was similar to the other renal metastatic cancer cell lines with cells preferring the mesh scaffolds over the other morphologies. The integrin $\alpha 5$ which is commonly expressed by renal cell carcinomas leads to similar cell adhesion and motility as 786-O and ACHN [335]. The HEK-293, a normal epithelial cell line did not express any significant changes in cell proliferation between the different scaffolds. However, the cell interactions and morphology of the cells varied between each scaffold. This was in correlation to the findings by $\mathrm{Su}$ and colleagues where cells grown in 3D spheres acquired stemness properties [336]. Like most metastatic carcinoma cell lines, the lung carcinoma (A549) had a high cell proliferation in the mechanically compliant mesh scaffolds. The cells were restricted to a small region of the honeycomb scaffold. This contradicted with the findings made by Tilghman et al. and Zhao et al. where there was higher proliferation on the stiffer substrate $[337,338]$. This difference in cell behavior in 2D vs 3D reiterates the importance of using 3D based culture methods and the role played by topographical cues.

The RT4 cells demonstrated differential cell shape and morphology which changed according to the scaffold on which cells were cultured [339]. The cells had a fibroblast like morphology on the aligned and honeycomb scaffolds with a papilloma like morphology on the mesh scaffolds on day 3 . The cell proliferation was highest on the aligned scaffolds. The motility of the cells was high when the anisotropy of the scaffolds provided contact guidance enabling cell spreading. The urothelial carcinoma T24 cancer cells are poorly differentiated and highly metastatic. The T24 cells expresses integrin $\alpha 5 \beta 1$ similar to the renal carcinomas which is necessary for cell adhesion, extravasation, and migration [340, 341]. The cells had higher proliferation and orientation on the aligned and honeycomb scaffolds but higher motility in the mesh scaffolds. These results complimented the 
research reported by Alfano and colleagues on the morphology of scaffolds necessary for bladder cancer cell invasion [342]. The mechanical preferences of the matrix were also reported by Raczkowska et al. [343]. The TCCSUP is a highly metastatic and differentiated bladder cancer cell line. There were no distinct differences in cell morphology between the cells seeded on different scaffolds and the cell proliferation rates. The cells spread uniformly on the scaffolds on all topographies but preferred the parts with mechanical strength (fig. S9). We hypothesize this to be due to an active YAP-induced oncogene addiction in cancer cells through the Hippo cascade causing loss of spatial preference and contact inhibition [344-346]. Thus, it can be concluded that the cells responded to the biophysical stimulus (geometrical cues and stiffness) with changes in cell morphology, proliferation, and motility. The scaffold provides a robust platform for design of novel tumor models, rapid screening of customized anti-cancer medications and therapies [294]. The ability to change topography without altering the surface chemical properties provides a holistic understanding of the driving mechanical factors such as tumor cell invasion, metastases, and survival.

\subsection{Conclusions}

Electrospun scaffolds of different morphologies were fabricated in a repeatable manner. The renal carcinoma cells seeded on the scaffolds favored the mesh scaffolds with the least mechanical strength. The ACHN cells had reduced migration from the pores of the honeycomb scaffold and subsequent reduction in cell proliferation rates. The HEK-293 cell line adapted to the different morphologies and no significant differences were found. The lung carcinoma cells proliferated well in the mesh scaffolds with a fibroblast like morphology on all the scaffolds. The RT4 cells demonstrated a fibroblast like morphology on the aligned scaffolds while epithelial morphology on the mesh scaffolds. The highly metastatic transitional carcinomas when poorly differentiated (T24) favored the mesh scaffolds with changes in cell morphology on the scaffold. However, when differentiated, (TCCSUP) they lost any contact inhibition or preference and proliferated well on stiffer matrices. The current findings lay the foundation to design in vitro tumor models and preferential inhibition of cell lines by altering the biophysical properties which in turn can lead to customized therapies and drug testing

Author Contributions: Samerender Nagam Hanumantharao- Conceptualization, visualization, methodology, software, validation, formal analysis, investigation, data curation, writing - original draft preparation; Carolynn Que- investigation, data curation, formal analysis, validation; Smitha Rao- resources, project administration, funding acquisition, supervision, writing - review and editing. All authors have read and agreed to the published version of the manuscript.

Funding: This work was supported by the financial support provided by the Department of Biomedical Engineering at Michigan Technological University, Portage Health Foundation (PHF) Research Excellence Fund Research Seed Grant for S. Rao. 
Acknowledgements: The authors would like to acknowledge the funding received through the Tech Talent Transfer award from the Michigan Economic Development Corporation for S. N. Hanumantharao; Portage Health Foundation (PHF) graduate assistantship for S. N. Hanumantharao, Doctoral finishing fellowship for Spring 2020 for S. N. Hanumantharao and financial support for C. Que through the PHF Research Excellence Fund Research Seed Grant for S. Rao.

Conflicts of Interest: The authors declare no conflict of interest. 


\subsection{Supplementary Information}

\section{Scaffold morphology affects proliferation of renal, lung and bladder cancer cells}

Samerender Nagam Hanumatharao, Carolynn Que and Smitha Rao*

Department of Biomedical Engineering, Michigan Technological University, Houghton, MI 49931, USA

*Correspondence: smithar@mtu.edu
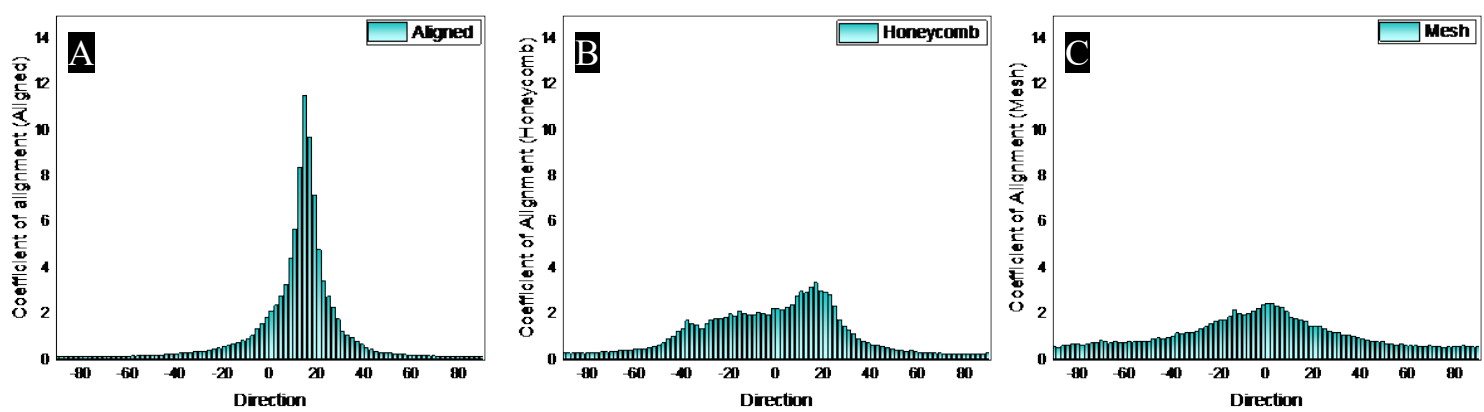

Fig. S1. The coefficient of alignment of all three scaffold morphologies (Aligned, Honeycomb and Mesh) were calculated using the Directionality plugin in Fiji from the FESEM image slices. The aligned scaffolds have a high density of fibers with defined orientations in a narrow range. This leads to peaks with low full width half maximums. The honeycomb scaffolds have their coefficient of alignment spread over a range of directions while the mesh scaffolds have no significant orientation.
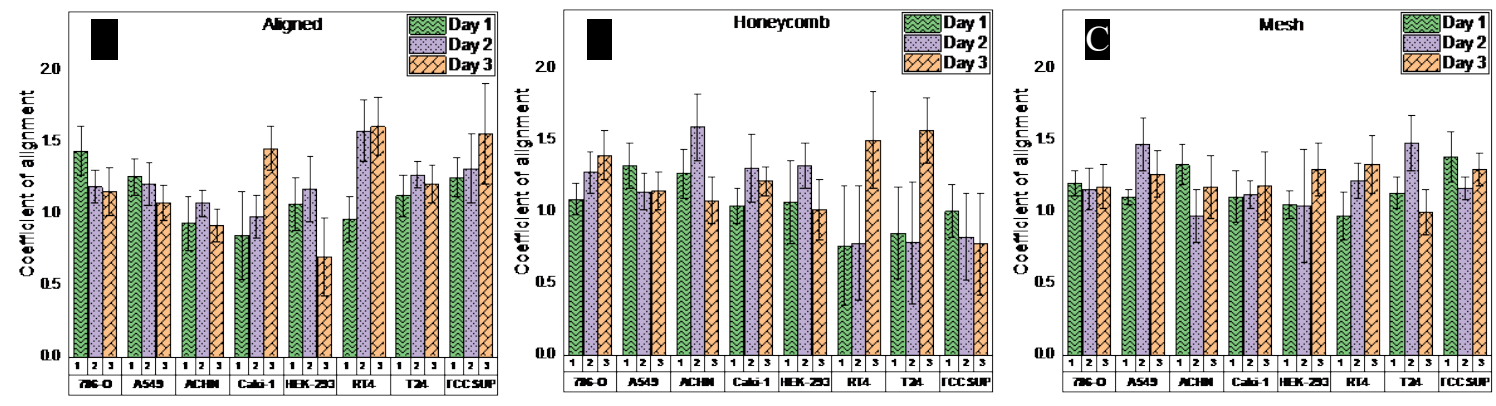

Fig. S2. The coefficient of alignment of cellular nuclei counter stained with DAPI on three scaffold morphologies on days 1, 2 and 3. A) Caki-1, TCCSUP and RT4 seeded on aligned scaffold had a significant increase in coefficient of alignment over three days. B) 786-O 
and RT4 seeded on honeycomb scaffolds had a significant increase in coefficient of alignment over three days while TCCSUP had a decrease over three days. C) RT4 seeded on mesh scaffolds had a significant increase in coefficient of alignment over three days. The alignment of 786-O and Caki-1 remained unchanged over the course of study.

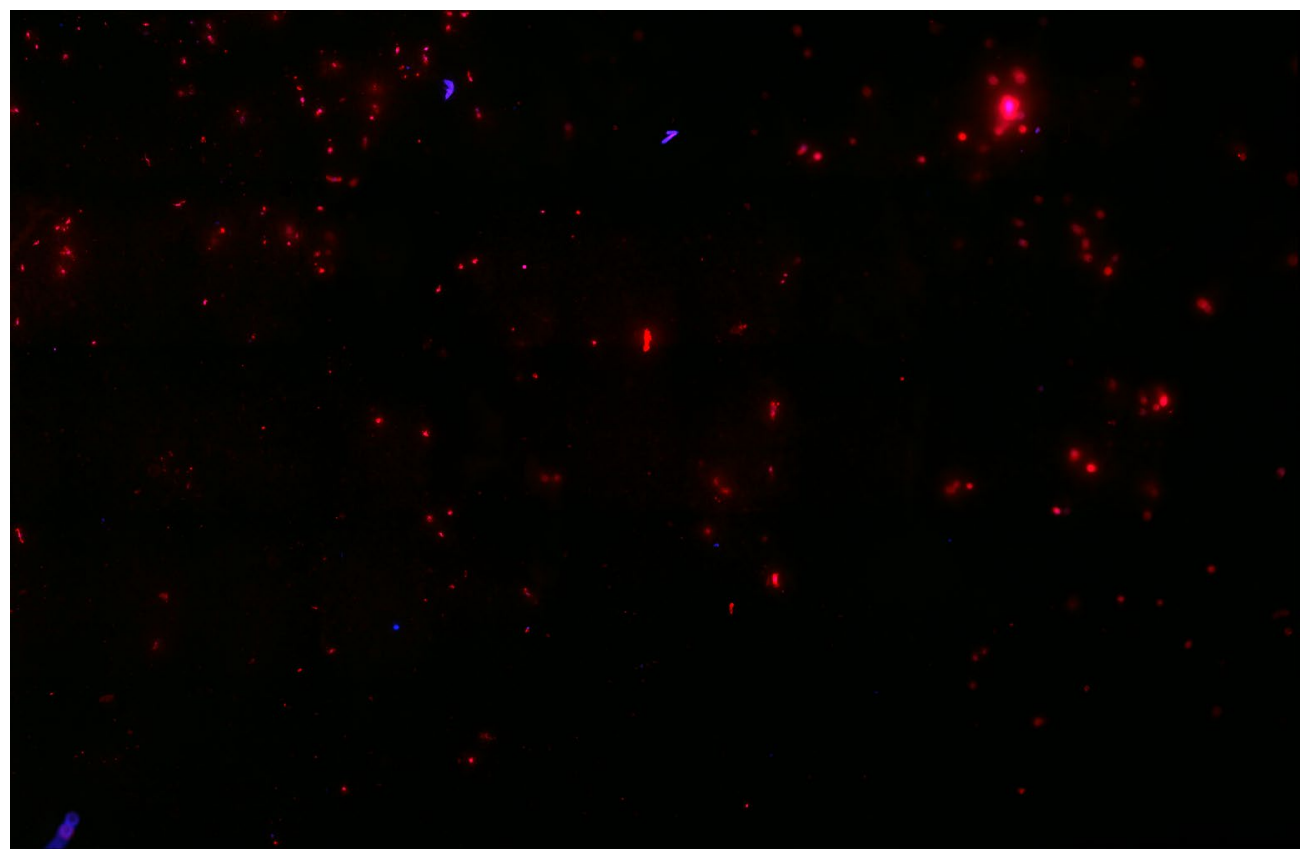

Fig. S3. A panoramic image of the fluorescent microscope images of renal adenocarcinoma cells $(786-\mathrm{O})$ on the mesh morphology $(1 \mathrm{~cm} \times 0.5 \mathrm{~cm})$ on day 3 . The nuclei were stained with DAPI (blue) and the actin filaments were stained with Alexa Fluor ${ }^{\circledR} 594$ Phalloidin (red). The cells migrated across the length and breadth of the scaffold.
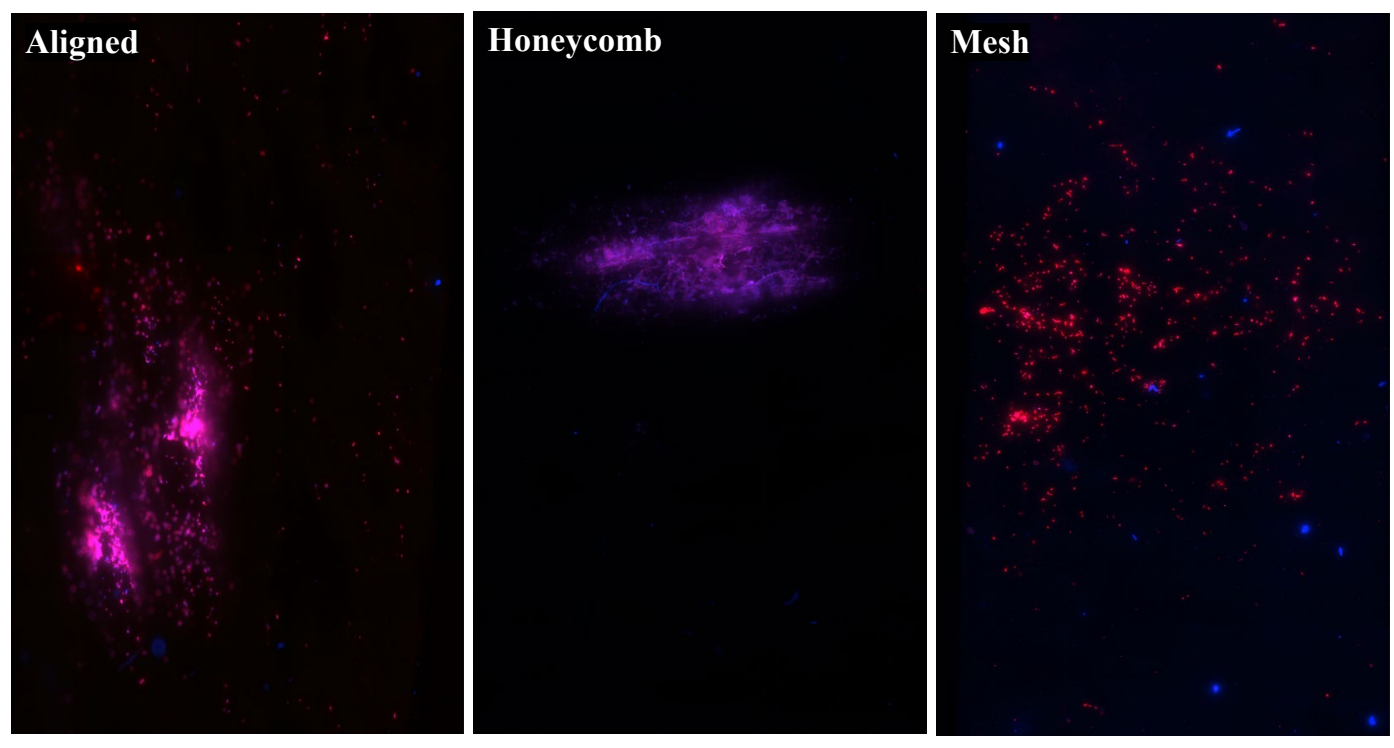
Fig. S4. A panoramic image of the fluorescent microscope images of renal adenocarcinoma cells $(\mathrm{ACHN})$ on the aligned, honeycomb and mesh morphology $(1 \mathrm{~cm} \times 0.5 \mathrm{~cm})$ on day 2 . The nuclei were stained with DAPI (blue) and the actin filaments were stained with Alexa Fluor ${ }^{\circledR} 594$ Phalloidin (red). The cells migrated along the orientation of the fibers in the scaffolds with aligned morphology. The cells were concentrated in a particular region of the honeycomb structure with little migration. The cells were uniformly distributed in the mesh scaffolds.
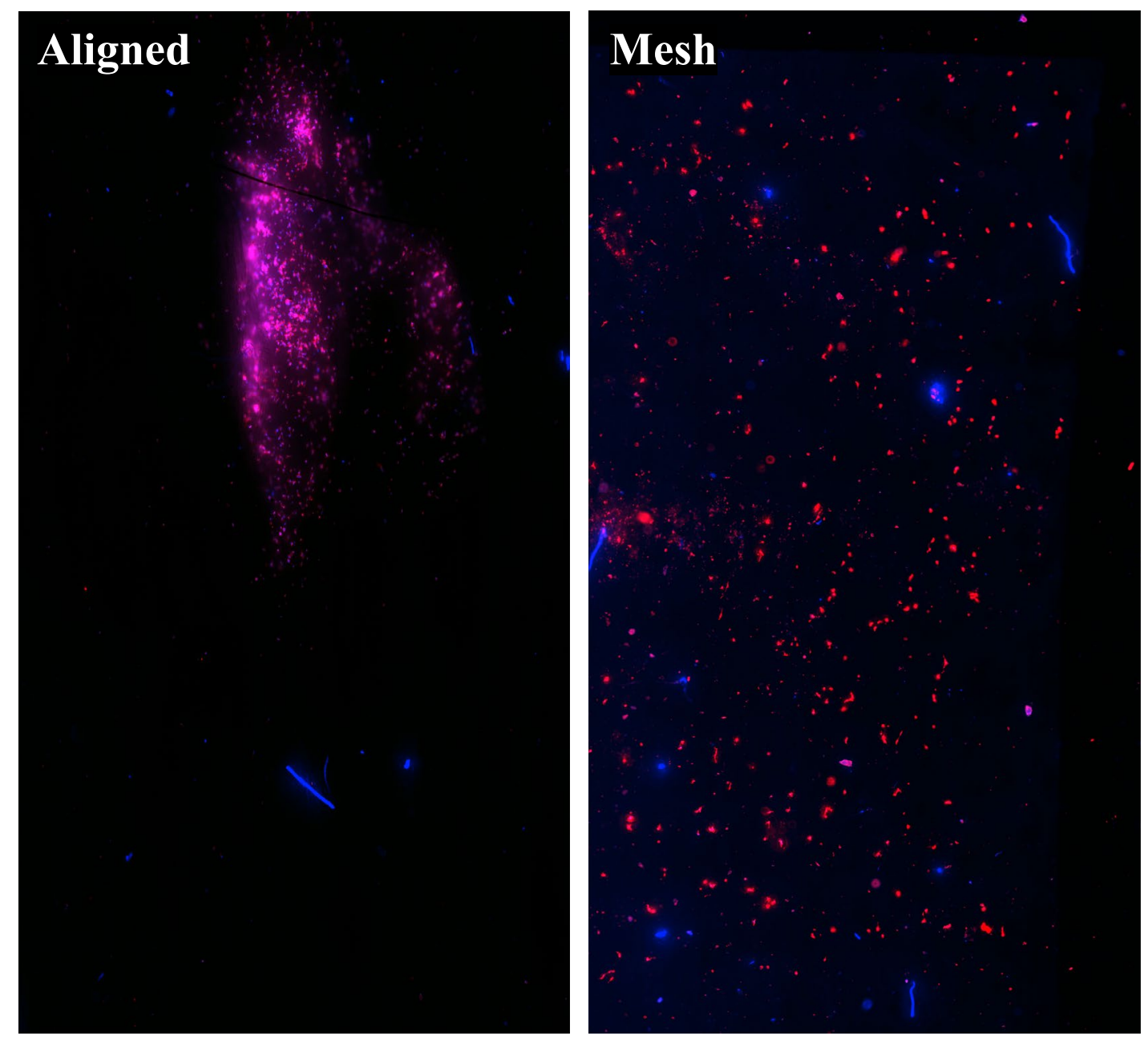

Fig. S5. A panoramic image of the fluorescent microscope images of renal adenocarcinoma cells $(\mathrm{ACHN})$ on the aligned and mesh morphology $(1 \mathrm{~cm} \times 0.5 \mathrm{~cm})$ on day 3 . The nuclei were stained with DAPI (blue) and the F-actin filaments were stained with Alexa Fluor ${ }^{\circledR}$ 594 Phalloidin (red). The differential preference of cells towards the topographical cues can be inferred based on the cell spreading pattern on the scaffolds. 


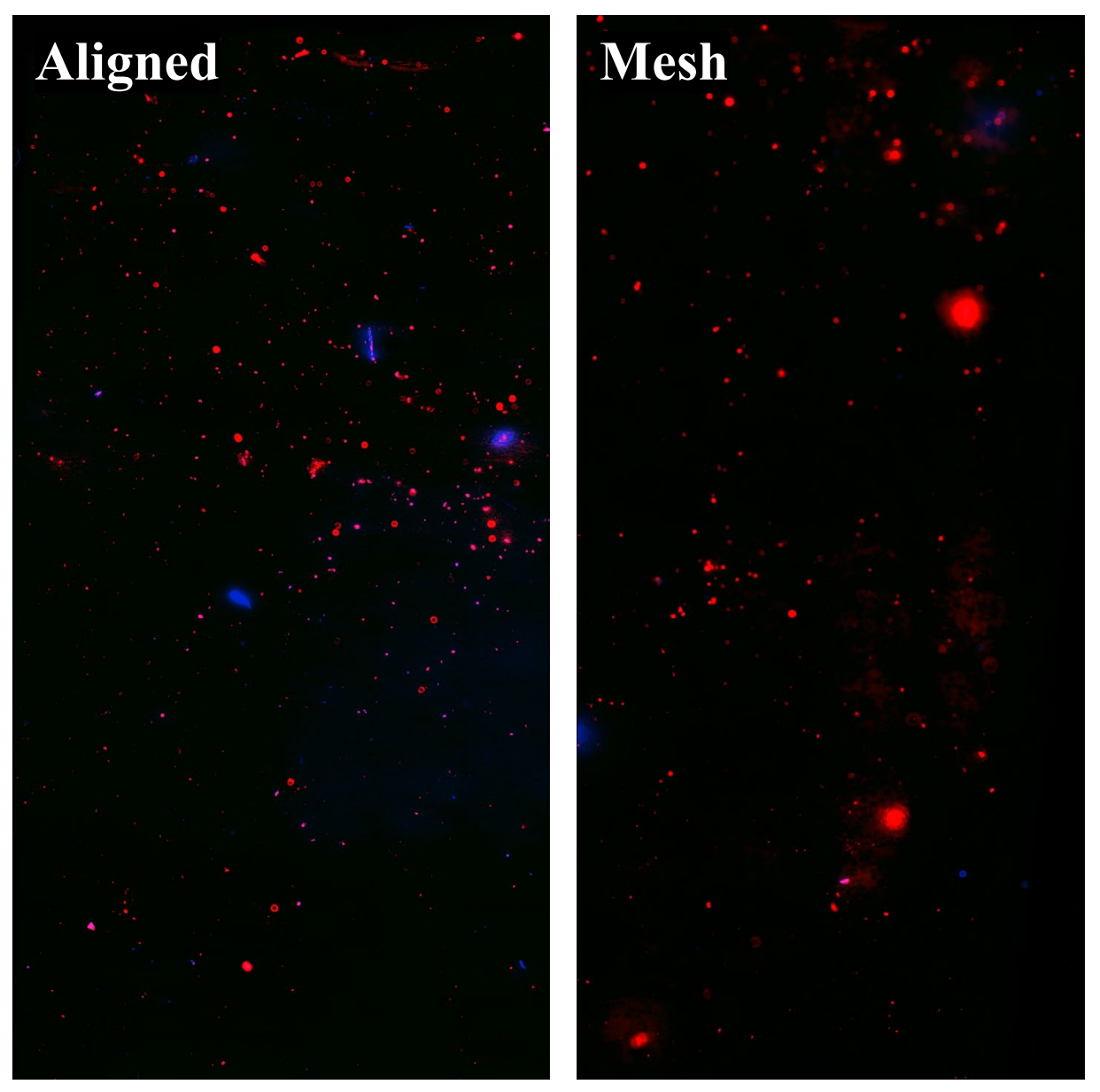

Fig. S6. A panoramic image of the fluorescent microscope images of Human Embryonic kidney epithelial cells (HEK-293) on the aligned and mesh morphology $(1 \mathrm{~cm} \times 0.5 \mathrm{~cm})$ on day 3. The nuclei were stained with DAPI (blue) and the F-actin filaments were stained with Alexa Fluor ${ }^{\circledR} 594$ Phalloidin (red). The cells did not have any specific preference toward a morphology of the scaffold. 


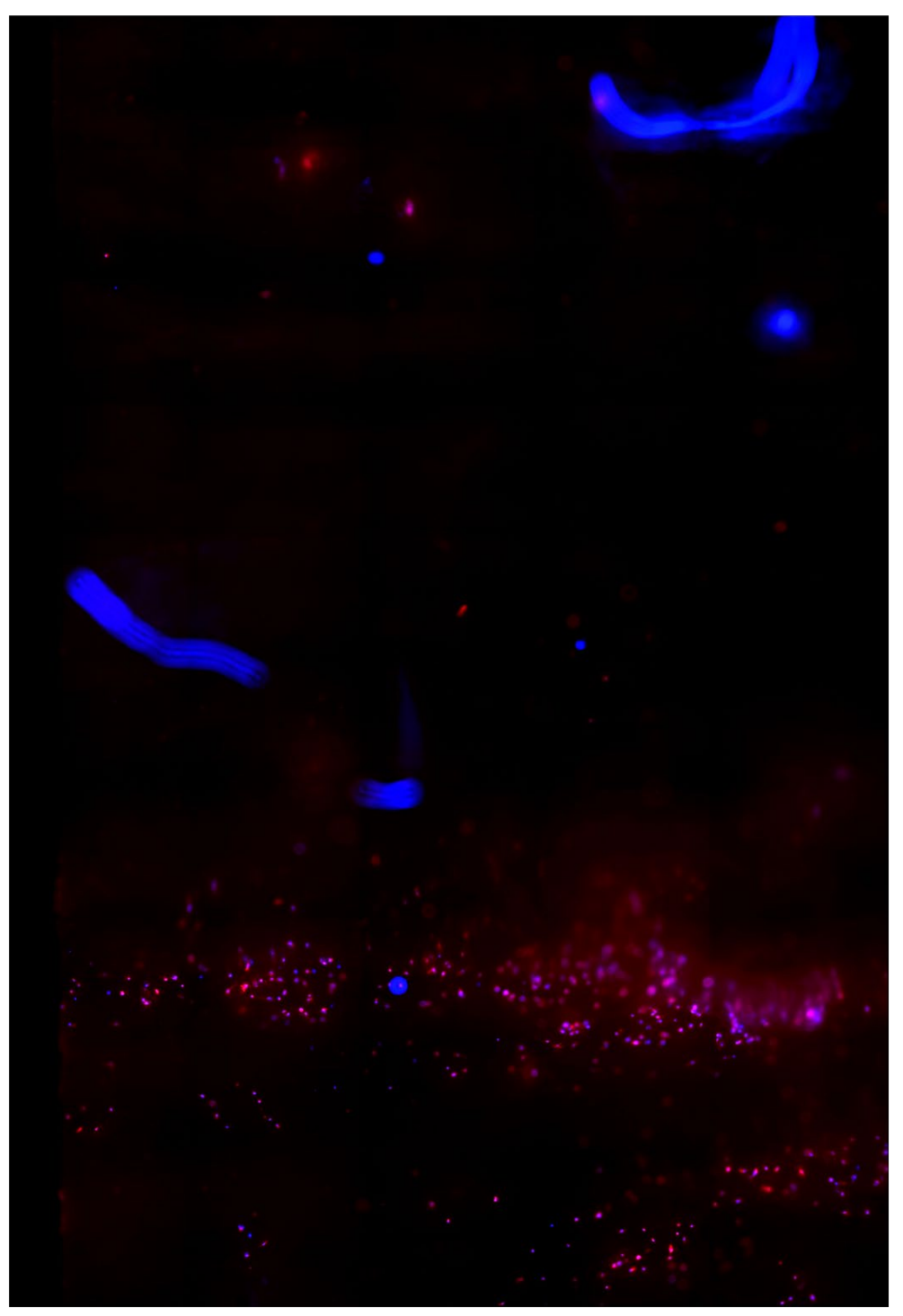

Fig. S7. A panoramic image of the fluorescent microscope images of lung carcinoma cells (A549) on the honeycomb morphology $(1 \mathrm{~cm} \times 0.5 \mathrm{~cm})$ on day 3 . The nuclei were stained with DAPI (blue) and the F-actin filaments were stained with Alexa Fluor ${ }^{\circledR} 594$ Phalloidin (red). The localization of cells in a small region of the scaffold and lack of spreading to other pores can be inferred. 

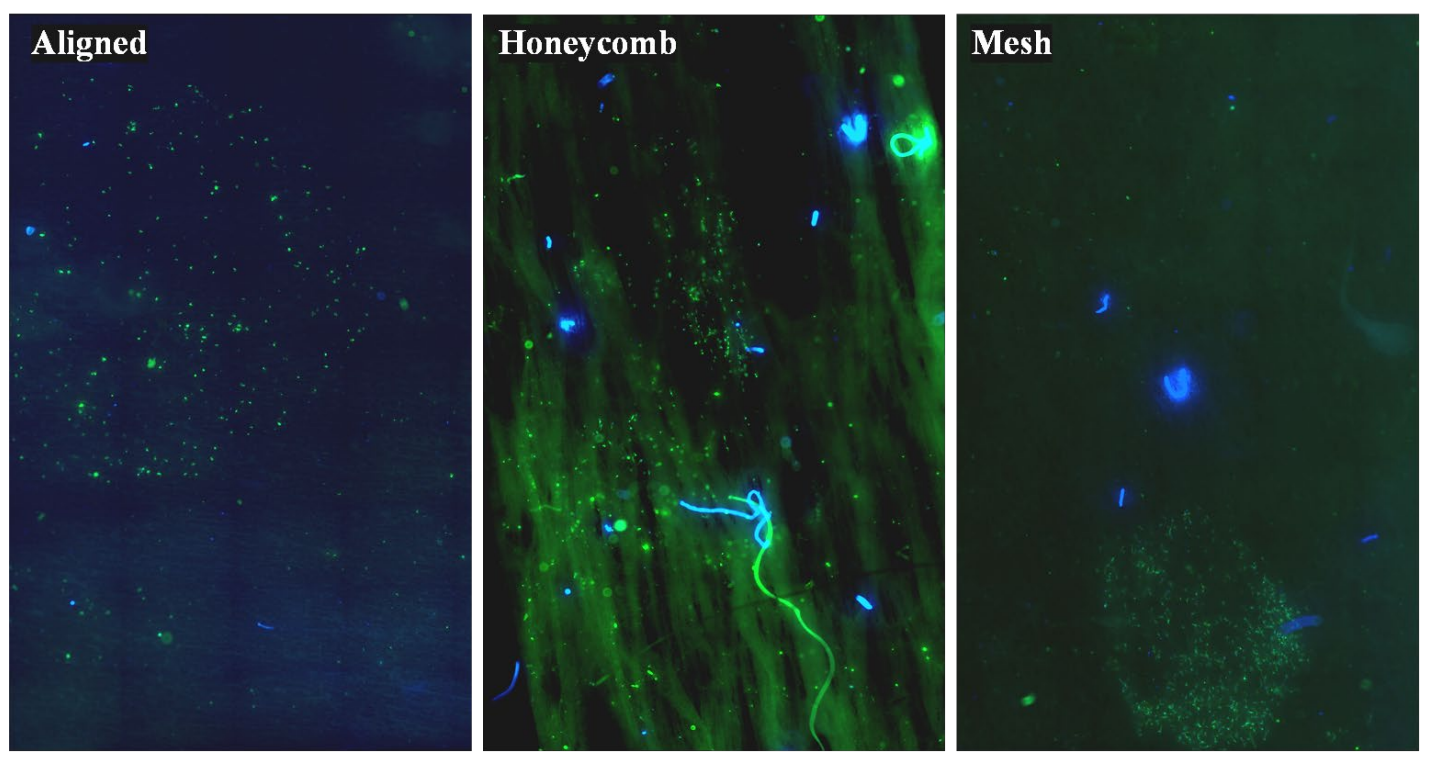

Fig. S8. A panoramic image of the fluorescent microscope images of transitional cell papilloma (RT4) on the aligned, honeycomb and mesh morphology $(1 \mathrm{~cm} \times 0.5 \mathrm{~cm})$ on day 3. The nuclei were stained with DAPI (blue) and the actin filaments were stained with Alexa Fluor ${ }^{\circledR} 488$ Phalloidin (green). Differential spreading of the cells can be seen on the three morphologies of the scaffold. The cells seemed to spread more on scaffolds which offer more contact guidance and have a greater orientation of fibers. 

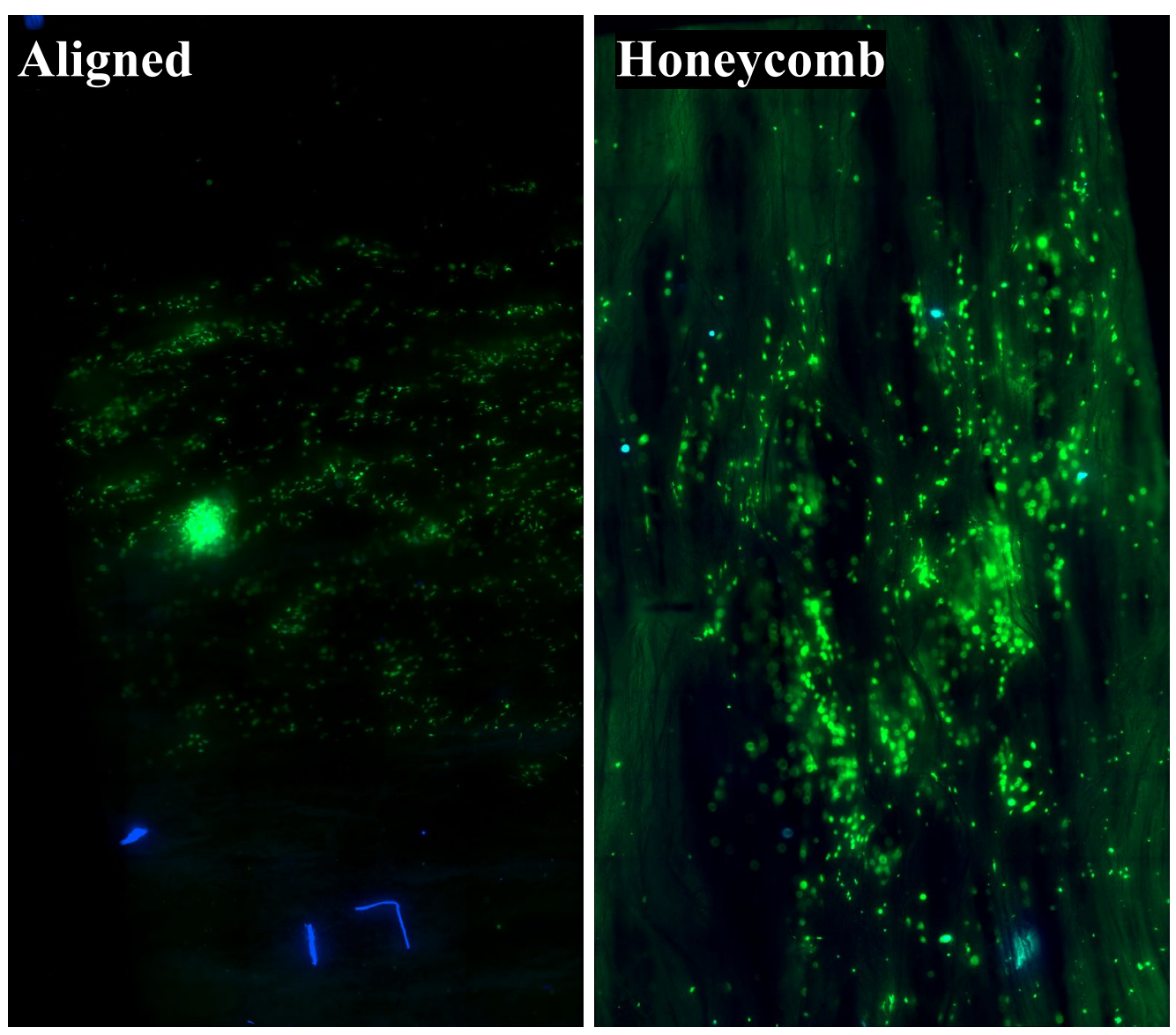

Fig. S9. A panoramic image of the fluorescent microscope images of bladder grade IV transitional cell carcinoma (TCCSUP) on the aligned and honeycomb morphology $(1 \mathrm{~cm} \times$ $0.5 \mathrm{~cm}$ ) on day 1 . The nuclei were stained with DAPI (blue) and the actin filaments were stained with Alexa Fluor ${ }^{\circledR} 488$ Phalloidin (green). The spreading of cells on the higher diameter fibers can be inferred based on the cell motility on the aligned and honeycomb scaffolds. 


\title{
6 A novel PCL scaffold based metabolic regulator in cancer cells
}

\author{
Samerender Nagam Hanumantharao ${ }^{1}$, Stephanie Bule ${ }^{1}$, Carolynn Que ${ }^{1}$, Brennan \\ Vogl', Marina Tanasova ${ }^{2}$, ${ }^{1}$ Smitha Rao ${ }^{1}$ \\ ${ }^{1}$ Department of Biomedical Engineering, Michigan Technological University, Houghton, \\ MI 49931, USA
}

${ }^{2}$ Department of Chemistry, Michigan Technological University, Houghton, MI 49931, USA

Corresponding author: *Email: smithar@mtu.edu

Keywords: Electrospinning; 3D scaffolds, metabolic reprogramming, Renal cancer, Breast cancer

\begin{abstract}
$\underline{\text { Abstract: }}$
Understanding the dynamic metabolic reprogramming of cancer cells is essential in understanding tumorigenesis. The presence of various metabolites and local biophysical cues influences the tumorigenesis process. In this study, we have used a GLUT5-specific fluorescent probe (ManCou-H) and fabricated scaffolds of different morphologies (aligned, honeycomb and mesh) from the same polymer solution for investigating the role of biophysical and metabolic cues on protein expression and metabolic activity of breast cancer cells. The study was also extended to lung and renal carcinoma cells. The internalization of the ManCou-H probes was analyzed across all the cell lines and changes in cell-cell as well as cell-matrix interactions were characterized visually. The cells responded to the metabolic and physical cues, which led to changes in protein expression in the breast cancer cells.
\end{abstract}

\subsection{Introduction:}

The increased metabolic requirement is a hallmark of cancer cells that has been well documented [347]. The complex microenvironments of tumors require flexibility in the metabolic pathways for the cells to proliferate and survive [348]. These adaptive mechanisms are commonly termed as metabolic reprogramming. The metabolic reprogramming of cells helps the cells utilize other metabolites apart from glucose and glutamine such as lactate, acetates, amino acids, polyamines, fatty acids, lipids, and exogenous proteins for energy acquisition, cell survival and biomass synthesis [349-352]. Metabolic reprogramming of cells involves multiple factors such as oncogenes, tumor suppressor genes and growth factors [353]. One of the first studies on increased oxygen use by tumor cells in comparison to normal cells was performed by Otto Warburg. The Warburg effect refers to the metabolic reprogramming of the glycolytic pathway increasing glycolytic flux. This in turn leads to several signaling cascades, one of which is the 
upregulation of the Glucose transporters (GLUTs) [354-357]. There are 14 known isoforms of GLUTs which can be separated into three classes based on their sequences [358-360]. The GLUTs have different affinities for carbohydrates and their location in the body is varied. Inhibition studies on GLUTs have resulted in apoptosis of cells. A recent study on gene set enrichment analyses found a correlation between the GLUTs and the YAP/TAZ pathway [361]. This cross-talk between the mechanotransduction pathways (YAP/TAZ, MAPK and P13K/Akt/mTOR signaling pathways) and metabolic reprogramming, including the role of GLUTs has been confirmed in vitro and in vivo [362366]. Although, the studies on GLUTs such as GLUT1 has been promising, their widespread expression in the body poses challenges in developing therapies or diagnostic targets. The fructose-specific transporter GLUT5 that has been shown to drive cancer metabolism in lung, renal and breast cancers [367-369].

In this paper, the relationship between mechanotransduction pathways and GLUT5 activity was leveraged to design novel scaffolds of different morphologies that release a GLUT5specific fluorescent probe regulating cell metabolism and altering cell behavior. The three morphologies of scaffolds used were based on our previously reported study [314]. The aligned scaffolds were designed to provide the highest contact guidance for cell elongation, migration and cell orientation. The honeycomb scaffolds provided tiny pockets that increase surface area available to the cells and also provide a porous structure for efficient cell infiltration through the layers of the scaffold. The mesh scaffolds were designed to mimic the natural epithelial basal layer with an interconnected network. The use of the fluorescent ManCou-H probes provides a visual representation of the metabolic profiles of cells. The presence of the ManCou-H probes along with the biophysical cues provided by the scaffolds helps to regulate the metabolic reprogramming of the cells. The current method opens up avenues for developing diagnostic and chemotherapeutic options similar to the 'metabogenic' regulation discussed by Ma and colleagues [370].

\subsection{Materials and Methods:}

\subsubsection{Synthesis of GLUT5-specific probe}

The GLUT5-specific probes were synthesized according to the previously reported work [371]. The conjugation of 2,5-anhydro-D-mannitol (2-AM) to an aromatic moiety such as coumarin with $\mathrm{H}$ in the $\mathrm{C} 4$ position formed the ManCou-H probes[372, 373]. The probes were used at a dilution of $20 \mu \mathrm{M}$ for cell culture experiments and for scaffold fabrication. The concentration used was based on previously published results on uptake studies performed in our lab on breast cancer cell lines using the ManCou-H probes[373]. The excitation and emission wavelengths of the ManCou-H probe used for visualization is 366 $\mathrm{nm}$ and $452 \mathrm{~nm}$ respectively. 


\subsubsection{Fabrication and characterization of scaffolds}

Polycaprolactone (PCL: Mw 70,000 GPC; Scientific Polymer Products, USA) dissolved in chloroform (Sigma Aldrich, USA) at a concentration of $20 \%$ weight was used for electrospinning with parameters and conditions as described in our previous work for fabrication of scaffolds of three distinct morphologies [314]. The schematic representation of the electrospinning setup is shown in figure 1A. The parameters varied were voltage, rotational speed and polymer injection volume rate in the following ranges $10 \mathrm{kV}$ to $11 \mathrm{kV}$ ,150-300 rpm and 180-240 $\mathrm{\mu l} / \mathrm{hr}$ respectively. Scaffolds containing ManCou-H with different morphologies were fabricated using the same parameters as described, by dissolving a $20 \mu \mathrm{M}$ concentration of ManCou-H probe with the polymer prior to electrospinning. The scaffolds were visually characterized using a field emission scanning electron microscope (FESEM; Hitachi S-4700 FE-SEM) after sputter coating with an $\mathrm{Au} / \mathrm{Pd}$ target. The distribution of fibers in the scaffolds and surface profile were characterized from the FESEM images using the directionality and surface plot plugins in the Fiji software respectively using the same parameters for analysis [318]. Surface characterization of the scaffolds was carried using Attenuated Fourier Transform Infrared Spectroscopy (Thermo Scientific ${ }^{\mathrm{TM}}$, Nicolet ${ }^{\mathrm{TM}}$ iS50 FTIR Spectrometer) from 400-4000 $\mathrm{cm}^{-1}$ at a resolution of $4 \mathrm{~cm}^{-1}$ with 256 scans. Multiple scans were performed at different regions of the scaffold to check for the presence of ManCou-H probes.

\subsubsection{Degradation Studies}

The degradation studies for the scaffolds with and without ManCou-H probes was performed to characterize the release of ManCou-H probes from the PCL scaffolds. Scaffolds with side $1 \mathrm{~cm}(\mathrm{n}=3)$ were cut and placed in Phosphate Buffer Solution (PBS) at $37^{\circ} \mathrm{C}$. At time points 24,48 and 72 hours the solution containing the leachate was removed and saved at $4^{\circ} \mathrm{C}$ and replaced with fresh PBS and placed back in the incubator. The fluorescence intensity of the leached ManCou-H probe was analyzed using a plate reader (Beckman Coulter DTX 880 Multimode Detector) at $360 \mathrm{~nm}$ excitation and $425 \mathrm{~nm}$ emission. A calibration curve was plotted using known concentrations of ManCou-H in PBS measured at $37^{\circ} \mathrm{C}$. The cumulative concentration of probe leached was estimated from the fluorescent intensities of the leached solution and the calibration curve.

\subsubsection{Cell Culture}

Adult human dermal fibroblasts (HDFa / ATCC ${ }^{\circledR}$ PCS-201-012 ${ }^{\mathrm{TM}}$ ), adenocarcinoma (MCF7/ATCC ${ }^{\circledR}$ HTB-22TM), triple negative malignant cells (MDA-MB-231 / ATCC ${ }^{\circledR}$ HTB-26 ${ }^{\mathrm{TM}}$ ), human breast epithelium (184B5 / ATCC ${ }^{\circledR}$ CRL-8799TM), clear cell renal carcinoma (Caki-1/ATCC ${ }^{\circledR}$ HTB-46 $6^{\mathrm{TM}}$ ), renal cell adenocarcinoma (786-O /ATCC ${ }^{\circledR}$ CRL$1932^{\mathrm{TM}}$ and ACHN/ATCC ${ }^{\circledR}$ CRL-161 ${ }^{\text {TM }}$ ), lung carcinoma (A549/ATCC ${ }^{\circledR}$ CCL-185 ${ }^{\mathrm{TM}}$ ) and human embryonic kidney epithelial cells (HEK-293/ATCC ${ }^{\circledR}$ CRL-1573 ${ }^{\mathrm{TM}}$ ) were purchased from America Type Cell Culture (ATCC). The premalignant cancer cell line (MCF10AneoT) was acquired from the Animal Model and Therapeutics Evaluation Core 
(AMTEC), Karmanos Cancer Institute, Wayne State University. All cell lines were cultured in their respective cell culture medium as prescribed by the manufacturer and maintained at physiological conditions (Temperature: $37^{\circ} \mathrm{C}$, Relative humidity: $65 \%$ and $5 \% \mathrm{CO}_{2}$ ). The cells were used in viable culture passages and closely monitored for changes in cell morphology.

\subsubsection{Cell Seeding}

Cells in the log phase were trypsinized and seeded on the different morphologies of the scaffolds. The scaffolds were cut into squares of area $0.25 \mathrm{~cm}^{2}$ for cell metabolism studies and $0.5 \mathrm{~cm}^{2}$ for other in vitro experiments. The cells were seeded on the scaffolds and maintained in standard conditions over the course of the study. The cell seeding density used for live cell tracking and immunocytochemistry was 3000 cells per sample, while 1500 cells per sample were used for other cell lines and 2500 cells per sample were used for HDFa for cell metabolism and fluorescent staining studies.

\subsubsection{Live cell tracking}

A $5 \mu \mathrm{M}$ solution of Cell Tracker Red CMTPX (Life Technologies, USA) in serum free RPMI culture media and a $5 \mu \mathrm{M}$ solution Cell Tracker Green CMFDA (Invitrogen ${ }^{\mathrm{TM}}$ ) in serum free RPMI culture media or supplemented MEGM culture media was prepared. The cells on plates in log phase containing MCF7 and MDA-MB-231 cells were incubated for $45 \mathrm{~min}$ at $37^{\circ} \mathrm{C}$ in the cell tracker red solution, whereas the cell tracker green solution was added to 184B5 (MEGM) and MDA-MB-231 (serum free RPMI) cells in log phase on plates removing whole culture media. The cells were trypsinized and seeded on scaffolds, scaffolds with ManCou-H, scaffolds supplemented with $20 \mu \mathrm{M}$ ManCou-H in media and plates at a seeding number of 3000 cells per sample. All the cells were cultured in RPMI 1640 culture media [+] L-glutamine (10-040-CVR, Corning $®$, USA) supplemented with $10 \%$ fetal bovine serum (FBS; Life Technologies, USA) along with $1 \%$ penicillinstreptomycin (Life Technologies, USA) and incubated at $37^{\circ} \mathrm{C}$. The cells were visualized after 24 and 48 hours of cell seeding using a fluorescence microscope (EVOS FL Auto) with the excitation and emission wavelengths as specified by the manufacturer's protocol and 366/452 nm Excitation/Emission wavelengths for the ManCou-H probes, respectively. Cell tracker Red was visualized through the TxRed filter while the cells stained using Cell tracker Green were visualized using GFP filter. Additionally, the phase images of the cells on scaffolds or plates were obtained to get a better representation of the cell-scaffold interactions.

\subsubsection{Immunocytochemistry}

Breast cancer cells (MCF7 and MDAMB231) and normal breast epithelium (184B5) were seeded on the bare scaffolds, scaffolds supplemented with $20 \mu \mathrm{M}$ ManCou-H in media and plates $(n=3)$. The cells were fixed after 24 and 48 hours of cell seeding using $4 \%$ paraformaldehyde. A blocking solution composed of Triton ${ }^{\circledR}$ X-100 (9002-93-1; Alfa 
Aesar, USA), bovine serum albumin (Fischer Scientific, USA), and donor horse serum (Corning, USA) were used to block non-specific binding. The scaffolds were stained separately (separate samples) with primary antibodies Anti-Cytokeratin 18 antibody [C04] (Abcam (ab668)) and Anti-Glut5 Antibody (MA 1-036X, Thermo Fischer Scientific) and secondary antibody Alexa Fluor ${ }^{\circledR}$ Plus 488 (Invitrogen ${ }^{\mathrm{TM}}$, USA) for GLUT5 and Cytokeratin 18 according to the manufacturer's protocol. The samples stained with Cytokeratin 18 were also stained with NucRed ${ }^{\mathrm{TM}}$ Live 647 ReadyProbes $^{\mathrm{TM}}$ Reagent (Invitrogen ${ }^{\mathrm{TM}}$, USA) according to manufacturer's protocols. The samples were imaged using a fluorescence microscope (EVOS FL Auto) with the excitation and emission wavelengths as specified by the manufacturer's protocol. Images were obtained with constant exposure, light, and gain settings at different regions of the scaffold randomly $(n=3)$. The images were processed using the Fiji software to calculate the corrected total cell fluorescence (CTCF) from randomly selected regions of interest for each image $(n=5)$ to obtain quantitative protein expression of GLUT5 and Cytokeratin 18 in cells [318]. Alternatively, qualitative images were also obtained to better visualize the data.

\subsubsection{Fluorescent Staining}

The cells seeded on the scaffolds with ManCou-H probes were fixed on days 1, 2 and 3 after cell seeding using 4\% paraformaldehyde, permeabilized with Triton-X 100 and stained with DAPI (4',6-diamidino-2-phenylindole) (Life Technologies, USA) and Alexa Fluor ${ }^{\circledR} 594$ Phalloidin (Invitrogen, USA) in HEK-293, Caki-1, ACHN, 786-O, A549, 184B5, HDFa, MCF7, MDAMB231 and MCF10AneoT cells according to the manufacturer's protocols. The cells on scaffolds without probes were also stained with the above dyes for the HDFa and 184B5 cells.

\subsubsection{Cell metabolism:}

The metabolic activity of cells leads to an irreversible reduction of redox sensitive resazurin (CellTiter-Blue ${ }^{\circledR}$ Cell Viability Assay (Promega, Madison, WI)) to fluorescent resorufin. The fluorescent intensity of resorufin was used to quantify the metabolic activity of cells seeded on scaffolds containing ManCou-H probes and plates on days 1, 2 and 3 after cell seeding $(\mathrm{n}=9)$. The cells cultured on standard tissue culture plates in similar conditions were used as a positive control ( $\mathrm{n}=6$ for HEK-293, Caki-1, ACHN, 786-O, A549 and $\mathrm{n}=3$ for 184B5, HDFa, MCF7, MDAMB231, MCF10AneoT). The relative fluorescence intensity was analyzed using a Beckman Coulter DTX 880 Multimode Detector at $560 \mathrm{~nm}$ excitation and $590 \mathrm{~nm}$ emission.

\subsubsection{Statistical Analysis}

The statistical analysis of the cell metabolism and protein expression of GLUT5 and Cytokeratin 18 was done using OriginPro 2018b and IBM ${ }^{\circledR}$ SPSS statistics V25 and represented as mean \pm SEM. The degradation data is represented as mean \pm standard deviation. The Wilcoxon non-parametric test was used to analyze statistical differences 
between $24 \mathrm{~h}$ and $48 \mathrm{~h}$ for each condition. The statistical differences between different days for each cell line and scaffold morphology was calculated using One-way ANOVA followed by post-hoc Tukey's test to calculate significance at $\mathrm{p}<0.05$.

\subsection{Results}

A

B
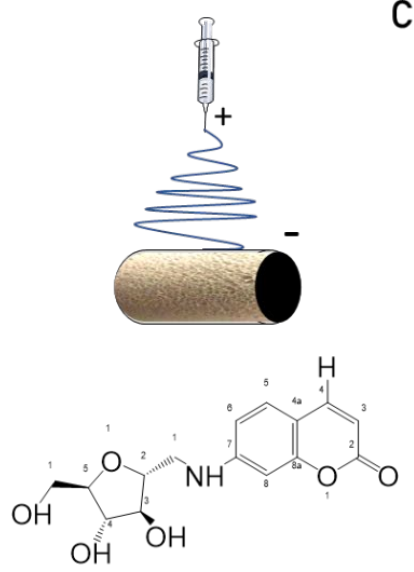

2,5-anhydro-D-mannitol-4H-coumarin

D

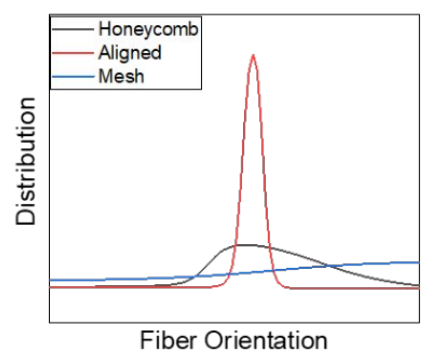

C
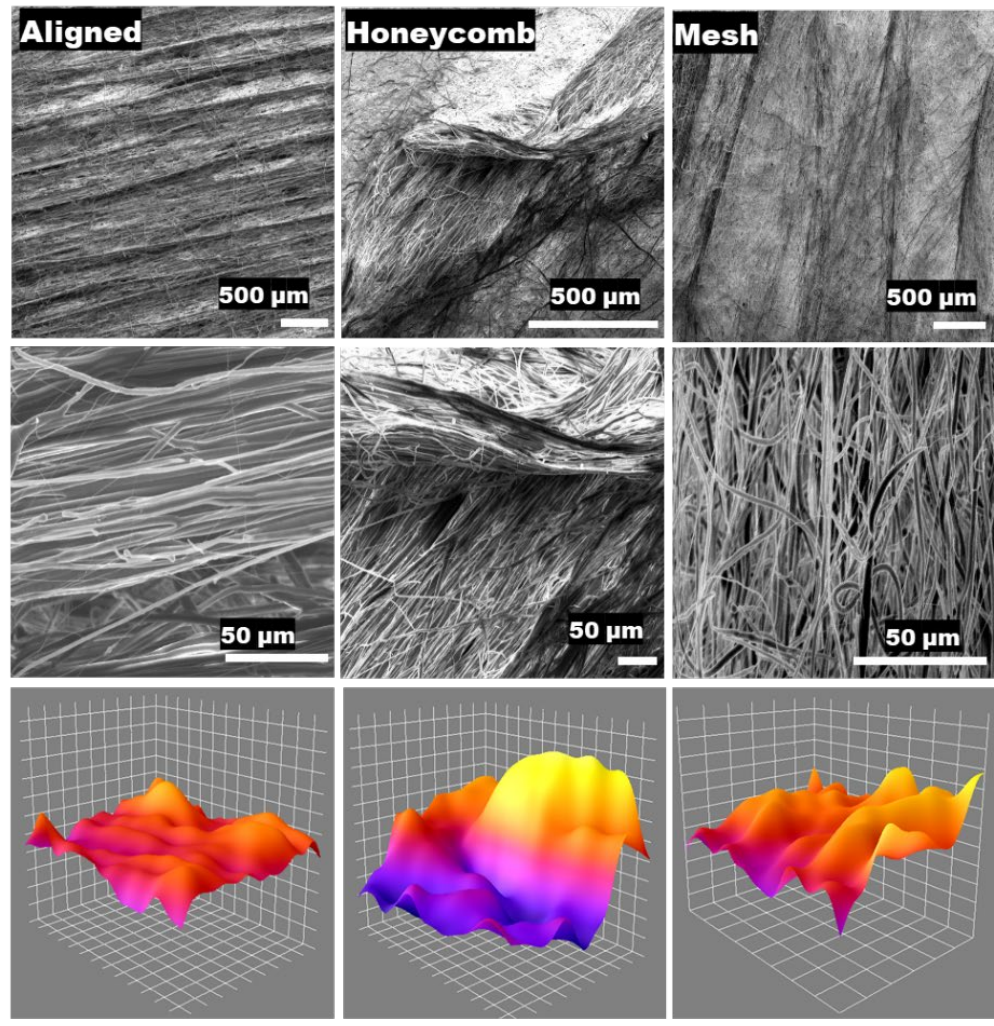

Figure 1A) A schematic representation of the electrospinning process used for the fabrication of scaffolds of different morphologies. B) Chemical structure of the fluorescent ManCou-H probes C) Field emission electron microscopy images of the aligned, honeycomb and mesh morphologies of the scaffolds. Images on the top are the low magnification images while the images in the middle are the high magnification images. The corresponding surface profile of the scaffolds is represented below to help visualize the $3 \mathrm{D}$ topography of the scaffolds.

\subsubsection{Scaffold fabrication and characterization}

A stable sol-gel composed of PCL and ManCou-H probes was used for electrospinning scaffolds with ManCou-H probes of three distinct morphologies- aligned, honeycomb and mesh. The same parameters were used for fabricating scaffolds of similar morphologies without probes. These scaffolds were used as control or were used after supplementing with ManCou-H in culture media. The repeatability of electrospinning helped fabricate 
scaffolds with similar morphologies multiple times as discussed in our previous publication [314]. From the FESEM images (figure 1C), the difference in surface topographies can be inferred. The aligned scaffolds had fibers of different diameters oriented in the same direction. Bands of tightly packed larger diameter fibers were present interspersed with fibers of smaller diameter. A higher magnification image of the aligned scaffolds revealed the splitting of larger diameter fibers into smaller diameter fibers leading to the fibers overlapping into different layers. The porous honeycomb topography is clearly visible from the repeating units as seen in figure S1. The scanning electron microscopy of the individual porous subunit reveals an underlying alignment of fibers of similar diameter and fibers on top forming the honeycomb pattern. A closer look at the sides of the honeycomb structure reveals splitting of fibers leading to the honeycomb patterned topography. The mesh fibers had little or no alignment of fibers, with fibers of varying diameters crisscrossing over each other to form a tight network. A high magnification image of the mesh scaffolds revealed no alignment across different layers of the fibers. The distribution of the fibers (figure 1D) had the narrowest range in the aligned scaffolds with mesh scaffolds having a very broad distribution of fibers. The honeycomb scaffolds were composed of fibers with alignment over a set range of orientation consistent with the pattern made by the fibers in the scaffold. The surface plot of all the different morphologies of the fibers helps visualize the crest and troughs in a 3D pattern providing a better representation of the morphology of the fibers. The mechanical characterization of the scaffolds was analyzed and elaborated in our previous study [314]. The honeycomb scaffolds had the highest mechanical strength followed closely by aligned scaffolds. The mesh scaffolds had the least mechanical strength and had elastomer like properties.
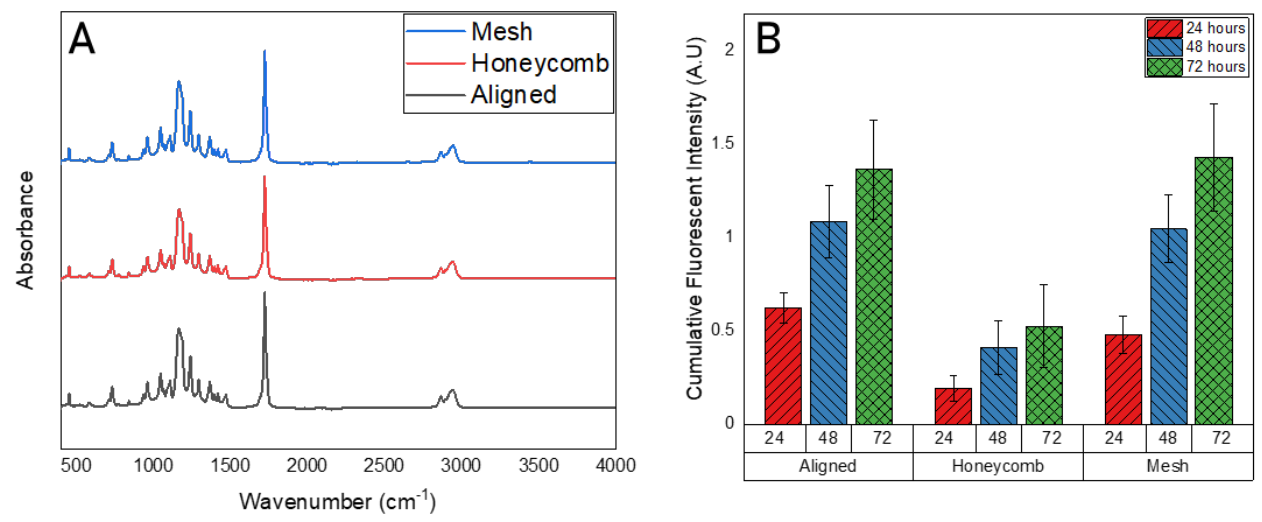

Figure 2 A) Surface spectra of the three scaffolds analyzed using ATR-FTIR. B) The cumulative fluorescent intensity caused due to the degradation of PCL scaffolds of different morphologies leading to release of ManCou-H probes.

The surface chemistry of all morphologies of the scaffolds was characterized using ATRFTIR (figure 2A). The scaffolds with different morphologies as inferred from the FESEM images had similar surface properties. The significant peaks corresponding to ManCou-H probes were absent when the FTIR spectrum was analyzed with the FTIR spectra from 
scaffolds with no probes. The presence of PCL seen from the strong absorbance at 2944 $\mathrm{cm}^{-1}$ and $2866 \mathrm{~cm}^{-1}$ are associated with asymmetric and symmetric stretching of the $\mathrm{CH}_{2}$ group. The multiple high absorbance peaks at $1470-1365 \mathrm{~cm}^{-1}$ was attributed to the $\mathrm{CH}_{2}$ and $\mathrm{CH}_{3}$, bending vibrations while peaks in the $961 \mathrm{~cm}^{-1}$ can be assigned to the bending of ester C-O-C in the trans configuration because of the crystallinity of PCL. The non-conjugated vibration due to the ester carbonyl group caused a strong absorbance at $1722 \mathrm{~cm}^{-1}$. The symmetric and asymmetric vibrations due to $\mathrm{C}-\mathrm{O}-\mathrm{C}$ stretching causes strong absorbance at $1239 \mathrm{~cm}^{-1}$ and $1164 \mathrm{~cm}^{-1}$. The crystalline phase of PCL (stretching of $\mathrm{C}-\mathrm{O}$ and $\mathrm{C}-\mathrm{C}$ at $1293 \mathrm{~cm}^{-1}$ ) is enhanced by electrospinning due to the high electrical fields used during fabrication of scaffolds [274].

\subsubsection{Degradation study}

The degradation study of the different morphologies of the scaffolds was performed to analyze the degradation of the scaffolds to release ManCou-H probes into the solution (figure $2 \mathrm{~B}$ and S2). PBS solution is used to mimic the physiological $\mathrm{pH} 7.4$ and the scaffolds were incubated at $37^{\circ} \mathrm{C}$. The scaffolds had different degradation profiles as analyzed and summarized in table 1 . The mesh scaffolds had the highest release of ManCou-H releasing $0.31 \mu \mathrm{M}$ after 72 hours in PBS while the aligned scaffolds leached out a cumulative total of $0.30 \mu \mathrm{M}$ in the same time frame. The honeycomb scaffolds had the lowest cumulative release of $0.009 \mu \mathrm{M}$ after 72 hours.

Table 1: Concentration of ManCou-H release at specific time points due to degradation of scaffolds

\begin{tabular}{|l|l|l|l|}
\hline Day & Aligned (in M) & Honeycomb (in M) & Mesh (in M) \\
\hline 1 & $1.117 \mathrm{E}-07$ & $3.9 \mathrm{E}-09$ & $7.54 \mathrm{E}-08$ \\
\hline 2 & $2.277 \mathrm{E}-07$ & $5.89 \mathrm{E}-08$ & $2.176 \mathrm{E}-07$ \\
\hline 3 & $2.972 \mathrm{E}-07$ & $8.72 \mathrm{E}-08$ & $3.135 \mathrm{E}-07$ \\
\hline
\end{tabular}

\subsubsection{Live cell tracking}

The breast cancer cells (MCF7 and MDAMB231) and normal breast epithelial cells (184B5) were used to track the changes in localization of cells, cell-scaffold behavior, and cell orientation for 24 and 48 hours (Figures S3-S33). The cells were seeded on standard tissue culture plates (control), scaffolds, scaffolds with ManCou-H probes and scaffolds supplemented with ManCou-H probes. Coculture studies of combination of two cell lines were also used to analyze changes in cell behavior and morphology. The cells on the plates for single cell and coculture studies had a very different morphology and cell localization 
on the matrix compared to the cells on scaffolds. The 3D structure of the scaffold played a major role in influencing the behavior of cells. There were no significant changes in cell morphology or behavior between the cells on scaffolds with ManCou-H probes and scaffolds supplemented with ManCou-H probes. Internalization of probe by the cancer cells caused localized clumping which was not present in the case of cancer cells on bare scaffolds.

\subsubsection{Protein expression}

The expression of Cytokeratin 18 and GLUT5 was analyzed in particular in breast cancer cells. Cytokeratin is a prognostic marker with a down-regulation leading to cancer progression [374] while GLUT5 is a fructose specific transporter that is over expressed in cancer cells $[375,376]$.

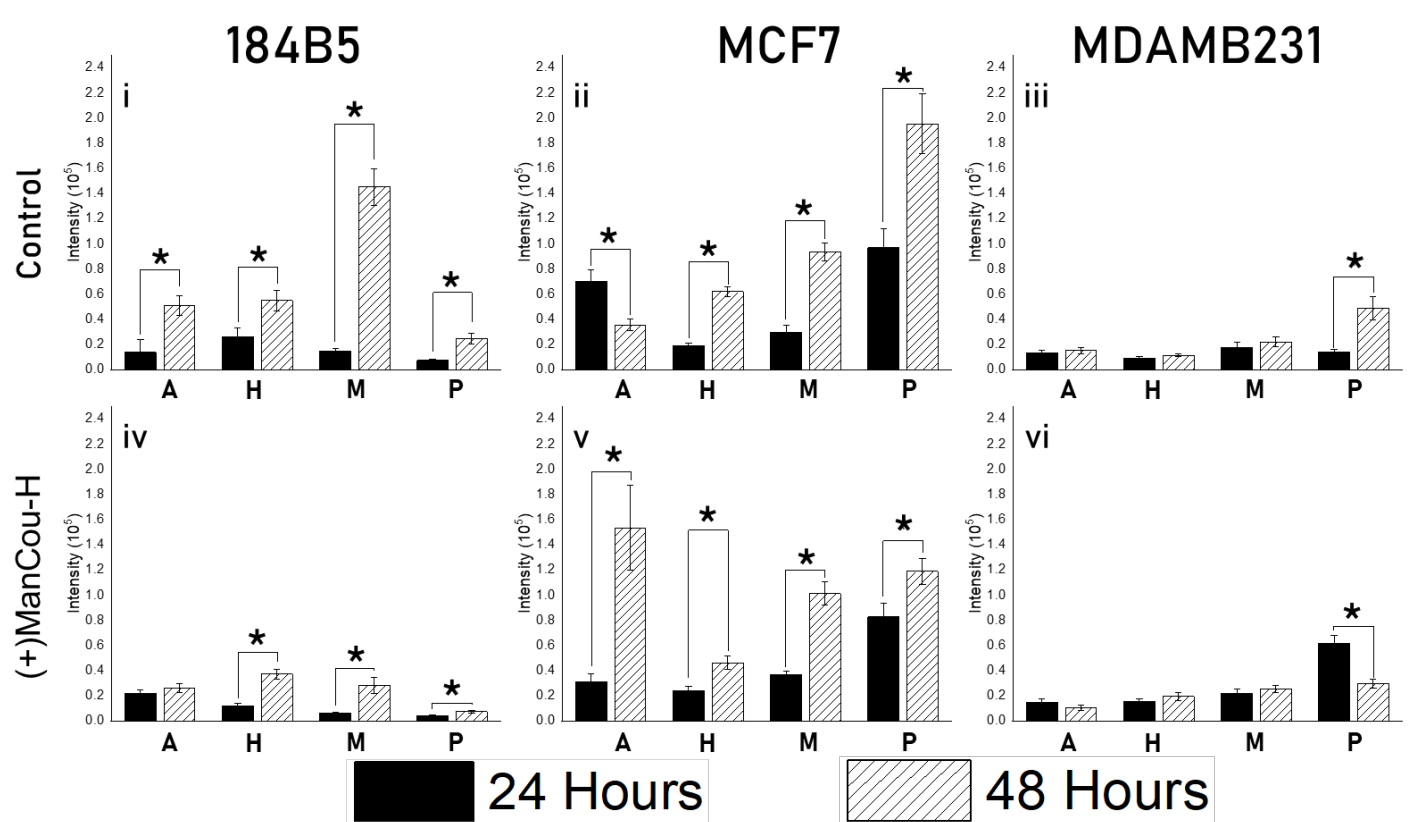

Figure 3 Protein expression of cytokeratin 18 on all three morphologies of bare scaffolds (i, ii, iii) and scaffolds supplemented with ManCou-H probes (iv, v, vi) over a period of 48 hours. A- Aligned scaffolds, H- Honeycomb scaffolds, M-Mesh scaffolds and P- tissue culture plates. Significance $\left.{ }^{*}\right)$ established at $\mathrm{p}<0.05$.

\subsubsection{Cytokeratin 18}

The expression of cytokeratin 18 was analyzed on cells seeded on scaffolds and scaffolds supplemented with $20 \mu \mathrm{M}$ ManCou-H probes after 24 and 48 hours of cell seeding (figure 3). Qualitative images of the cells help better visualization of the results (figure S39-S50). The normal breast epithelium had an increase in expression of cytokeratin 18 across morphologies of the scaffolds without probes and on plates. This increase was consistent 
on the normal breast epithelium when it was supplemented with ManCou-H probes. The cytokeratin expression decreased in the aligned scaffolds without probes but increased in the presence of the probes. The protein expression increased significantly across the mesh and honeycomb scaffolds and plate with and without ManCou-H probes. The relative expression of the protein was lower in the MDAMB231 cells as compared to the 184B5 and MCF7 cells. The addition of ManCou-H probe caused a reduced expression of the protein in the MDAMB231 cells compared to the absence of the probes.

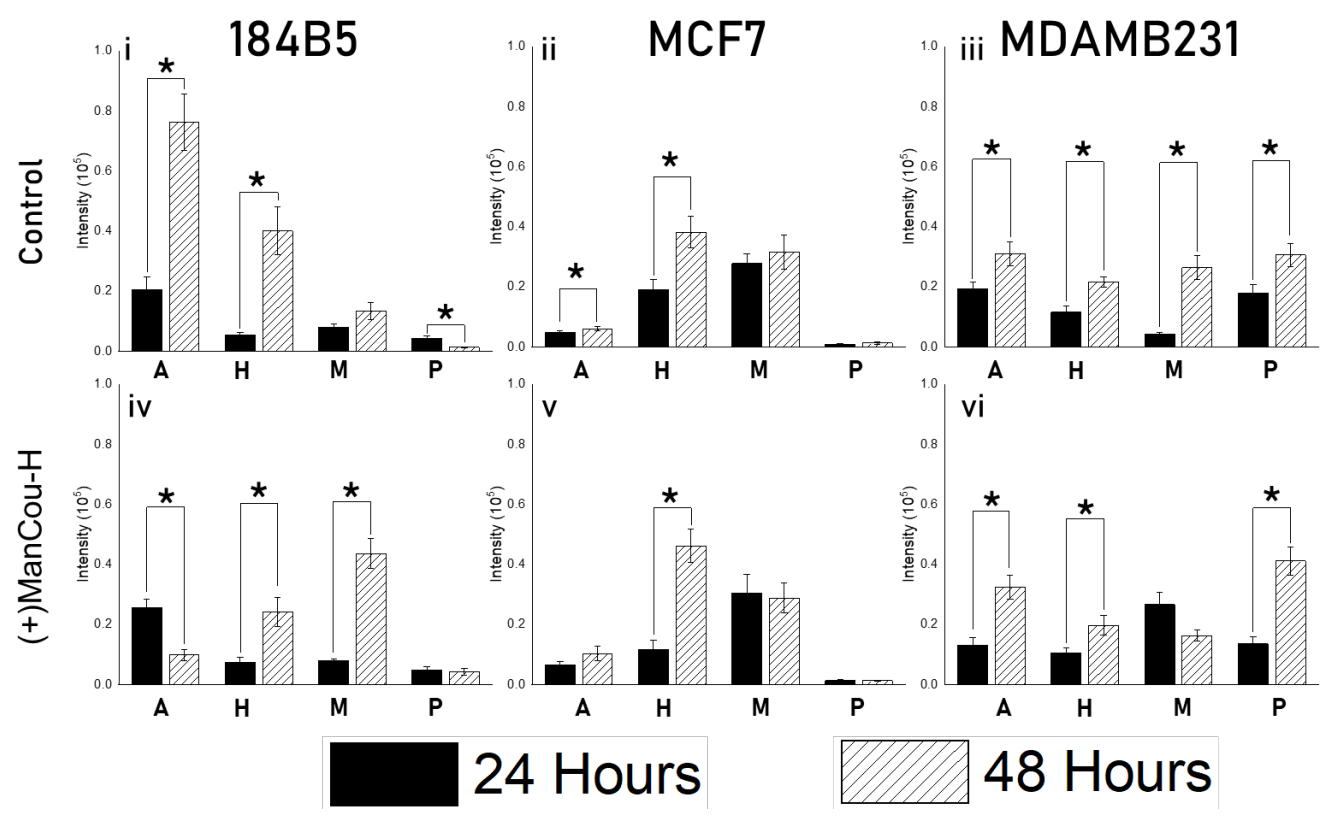

Figure 4 Protein expression of GLUT5 on all three morphologies of bare scaffolds (i, ii, iii) and scaffolds supplemented with ManCou-H probes (iv, v, vi) over a period of 48 hours. A- Aligned scaffolds, H- Honeycomb scaffolds, M-Mesh scaffolds and P- tissue culture plates. Significance $(*)$ established at $\mathrm{p}<0.05$.

\subsubsection{GLUT5}

Protein expression of GLUT5 was characterized on cells seeded on scaffolds and scaffolds supplemented with $20 \mu \mathrm{M}$ ManCou-H probes after 24 and 48 hours of cell seeding (figure 4). The qualitative images of the GLUT5 staining on cells provide a better representation of the quantitative results (figures S27-S38). The normal breast epithelium had an increase in expression of GLUT5 in the aligned and honeycomb scaffolds while there was a decrease in expression on plates. When supplemented with ManCou- $\mathrm{H}$, there was a decrease in GLUT5 activity in the aligned scaffolds but an increase in the honeycomb and mesh scaffolds. In the case of MCF7 cells, there was a significant increase in the aligned and honeycomb scaffolds and a significant increase in the honeycomb scaffolds when supplemented with the GLUT5 specific probe. The increasing trend in GLUT5 expression was similar in the MDAMB231 cells across all the scaffold morphologies and culture plate. 
When supplemented with ManCou-H, the trend was similar to conditions without ManCou-H probes, except for the mesh scaffolds.

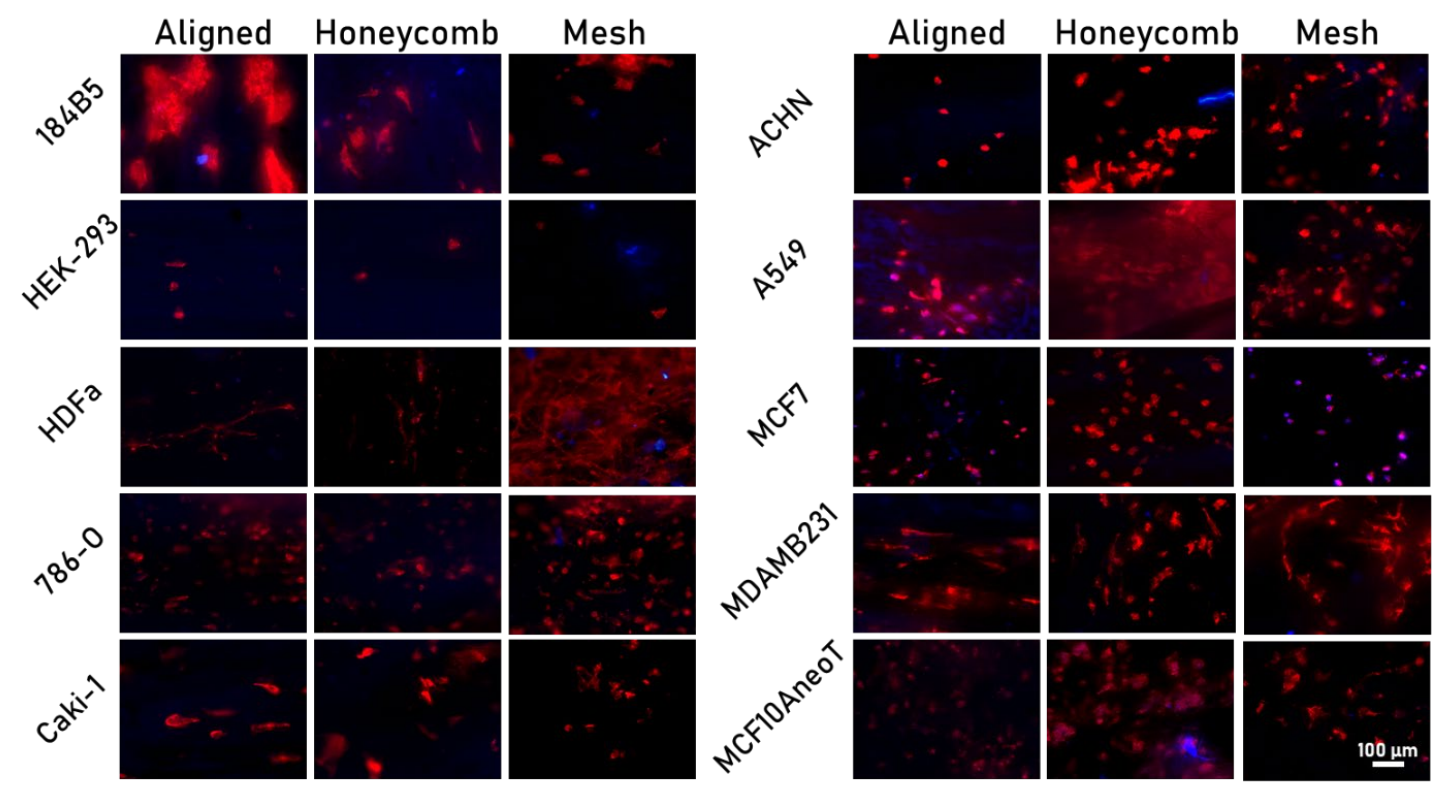

Figure 5 Fluorescent microscope images of cells seeded on scaffolds containing ManCou$\mathrm{H}$ probes on day 3 for 184B5, HEK-293, HDFa, 786-O, Caki-1, ACHN, A549, MCF7, MDAMB231 and MCF10AneoT cells. The probe is represented blue in color while the the F-actin filaments were stained with Alexa Fluor ${ }^{\circledR} 594$ Phalloidin (red). The internalization of the probes (blue) by the cells (red) and dispersion by the fibers causes some of the cells to appear pink in color. Images captured at $10 \mathrm{X}$ magnification.

\subsubsection{Fluorescent Staining}

The cells were fluorescently stained for the F-Actin filaments to visualize the cell-matrix and cell-cell interactions on scaffolds with ManCou-H probes (figure 5 and figures S51S62). The images from day 3 are present in the main paper while the images from the other days are present in the supplementary information. The normal breast epithelium (184B5) had cells in clusters in all morphologies of the scaffold. The cells preferred the aligned scaffolds where the cells were able to migrate due to contact guidance. The HEK-293 (embryonic kidney epithelial) cells had no preference and had the same morphology and cell behavior across all the scaffolds. The fibroblasts (HDFa) spread across the mesh scaffolds while the cells were more elongated in the aligned and honeycomb scaffolds. The 786-O (renal adenocarcinoma) cells were spread out evenly across all the scaffolds and had no distinct preferences to any of the scaffolds. The Caki-1 (clear cell renal carcinoma) cells were elongated on the aligned scaffolds and evenly spread on the other scaffold morphologies. The renal adenocarcinoma (ACHN) cells did not have any preference on the morphology of the scaffold. The A549 cells (lung carcinoma) cells penetrated through the different layers of the scaffold and spread uniformly across all the scaffolds. The breast 
adenocarcinoma cells (MCF7) internalized the ManCou-H probes as seen from the pink color due to the overlay of F-Actin and fluorescent probes. The cells were aligned along a similar orientation on the aligned scaffolds while the cells were uniformly spread across the different layers on the mesh scaffolds. The triple negative breast cancer cells (MDAMB231) did not have a specific preference on the morphology of the scaffold but were localized to the thicker diameter fibers which subsequently have the highest mechanical strength. The premalignant cancer cells (MCF10AneoT) were present in clusters on the honeycomb scaffolds. The cells were more elongated on the aligned scaffolds but were limited to the upper layer of the scaffolds. The cells infiltrated the lower layers in the honeycomb and mesh scaffolds.

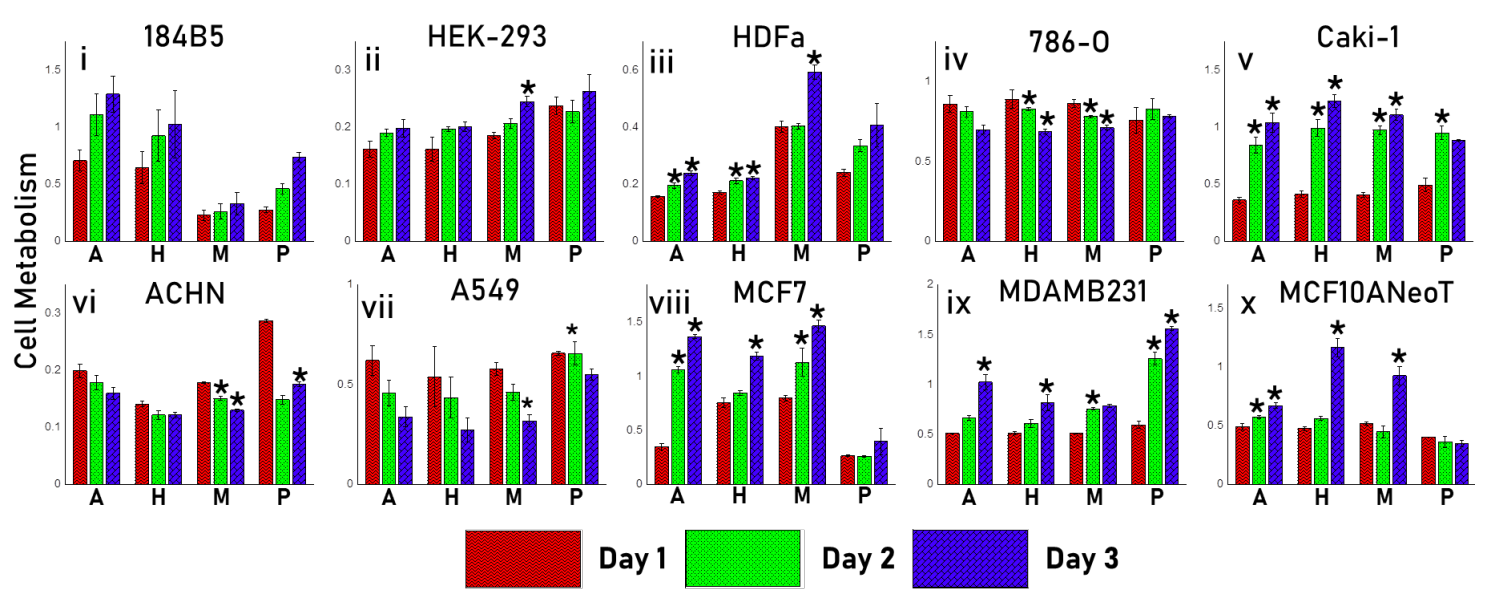

Figure 6 Quantification of proliferative cell metabolism on days 1, 2 and 3 on different scaffold morphologies. i) 184B5, ii) HEK-293, iii) HDFa, iv) 786-O, v) Caki-1, vi) ACHN, vii) A549, viii) MCF7, ix) MDAMB231 and $\mathrm{x}$ ) MCF10AneoT cells. A- Aligned scaffolds, H- Honeycomb scaffolds, M-Mesh scaffolds and P- tissue culture plates. Significance $\left({ }^{*}\right)$ established at $\mathrm{p}<0.05$.

\subsubsection{Cell Metabolism}

The cellular metabolism of all the cell lines were investigated on cells seeded on scaffolds with probes (figure 6 and figure S63). The cellular metabolism of the breast epithelial cells (184B5) were higher on the aligned and honeycomb scaffolds compared to the mesh and culture plates. The HEK-293 (embryonic kidney epithelial cells) cells had no preference and had a similar metabolic rate across all the scaffolds. The fibroblasts (HDFa) had the highest metabolic rate on mesh scaffolds. The 786-O (renal adenocarcinoma) cells had no preference and had a similar metabolic rate across all the scaffolds. The Caki-1 (clear cell renal carcinoma) cells had higher metabolic activity on 3D scaffolds than culture plates. The renal adenocarcinoma (ACHN) cells and the lung carcinoma (A549) had the highest metabolic activity on cell culture plates. The breast adenocarcinoma (MCF7) cells had higher metabolic activity on 3D scaffolds than culture plates. The metabolic activity was highest on the mesh scaffolds closely followed by the aligned scaffolds. The triple negative 
breast cancer cells (MDAMB231) had a preference for matrix with a high mechanical strength. The cells had the highest metabolic rate on the cell culture plates followed by the aligned and honeycomb scaffolds. The premalignant cancer cells (MCF10AneoT) had the highest metabolic rates on the honeycomb scaffolds followed by the mesh and aligned scaffolds respectively.

\subsection{Discussion}

The sol-gel preparation and parameters used for electrospinning enabled the fabrication of PCL scaffolds of different morphologies (mechanical properties and topography) but similar surface properties. However, the probes present in the scaffold eluted out of the scaffolds at different rates. This is because of the different parameters used during the electrospinning process which affects the localization of probes in the fibers, the density of the fibers for a given morphology and the diameter of the fibers [377]. The altered metabolic reprogramming in cancer leads to many changes in the dividing cells including changes in cell morphology. The live tracking of cells on scaffolds, scaffolds supplemented with ManCou-H, scaffolds with ManCou-H was performed to check differences in cell phenotype in each condition over a period of 48 hours. The effect of ManCou-H on cells was calculated to be $20 \mathrm{~min}$ from our previous experiments [373]. The time point for the current study was chosen to study the short-term effects on biomaterial based metabolic cues on cells which in turn leads to other long term effects [378]. The cells did not have any changes in morphology compared to the scaffolds, scaffolds with ManCou-H and scaffolds supplemented with ManCou-H. Further analyses on protein expression was done using scaffolds supplemented with ManCou-H to closely monitor the effect of metabolites (ManCou-H) on biophysical cues and ensuring uniform bioavailability of probes. The protein expression of Cytokeratin 18 did not vary in the 184B5, normal breast epithelium when supplemented with probes. However, there were changes in the case of MCF7 cells where the protein expression differed in the presence and absence of ManCou-H probes on select morphologies. The protein was also downregulated in the highly metastatic breast cancer cells (MDAMB231), where there was a statistically significant change in protein expression between the tissue culture plates. The upregulation of the fructose transporter GLUT5 is part of the metabolic reprogramming of cancer cells to adapt to their growing energy needs. This reprogramming was evident in the case of MCF7 cells on aligned morphology and the MDAMB231 cells on mesh scaffolds. The mechanosensation by cells occurs almost immediately as shown by Strohmeyer and colleagues [325]. Alternatively, an inverse relationship can also be inferred based on the protein expression profiles of MCF7 cells where a high expression of GLUT5 alone did not lead to cytokeratin downregulation in the case of honeycomb scaffolds. There were no significant changes seen in the MDAMB231 cells because of the expected hyperactivation of YAP [344]. A qualitative analysis of the cell phenotypes and internalization of the fluorescent probes by select cell lines on select scaffold morphologies demonstrate the dynamic changes in cell behavior. The reprogramming of the cells due to the ManCou-H probes caused variations in the proliferative metabolism of the cells leading to differences in cell metabolism across different types of scaffold morphologies for lung, breast and renal cancer cells. These 
regulations in metabolic reprogramming was distinctive to one specific morphology of the scaffold, as the other variables were constant.

The heterogeneity of the different types of cancers tested have been reported to exhibit a common finite set of signaling cascades which are positively influenced by the scaffold morphology and GLUT5 through the cross talk between metabolic reprogramming and mechanotransduction [379, 380]. The renal cancer cells and the lung cancer cells responded to the biophysical and metabolic cues with changes in cell metabolism across different scaffolds. The cellular senescence in the case of lung carcinomas is seen across all the scaffolds with probes. This behavior is also seen in the renal adenocarcinomas. Further research is needed to investigate into the role of GLUT5 and cellular senescence [381, 382]. The current biomaterial based metabolic regulator opens avenues for targeted cancer diagnosis, therapies and a tool for investigating long term effects of biomaterial based metabolic regulators in vivo.

\subsection{Conclusions}

Scaffolds with different morphologies (aligned, honeycomb and mesh) were fabricated with a fluorescent GLUT5 specific probe (ManCou-H). The slow degradation of PCL provided a controlled and slow release of the probes from the polymeric scaffolds. The localization of the probes varied between each morphology of the scaffold leading to differential release of the probes. The ManCou-H probe along with the scaffold lead to differential cell metabolism among scaffolds of different morphologies. The Cytokeratin 18 and GLUT5 expression profile was used to infer the effect of mechanotransduction and metabolic cues on breast cancer cells where the selective preference of scaffold morphology was inferred. The renal and lung carcinomas altered their cellular metabolism according to the scaffold. The current biomaterial-based cell regulator provides a tool for developing therapies.

\section{Competing interests}

The authors declare no competing or financial interests.

\section{$\underline{\text { Acknowledgements }}$}

This work was supported by the financial support provided by the Department of Biomedical Engineering at Michigan Technological University, the Tech Talent Transfer award from the Michigan Economic Development Corporation for S. N. Hanumantharao; Portage Health Foundation (PHF) graduate assistantship for S. N. Hanumantharao; financial support for C. Que through the PHF Research Excellence Fund Research Seed Grant for S. Rao; federal grant from National Cancer Institute (1R15CA242401-01) for S. Rao and M. Tanasova; financial support by Undergraduate Research Internship Program (URIP) and Summer Undergraduate Research Fellowship (SURF) at Michigan Technological University for B. Vogl. The authors would also like to 
acknowledge Dr. Kathryn Perrine, Department of Chemistry at Michigan Technological University for her help with surface characterization of scaffolds.

\subsection{Supplementary Information}

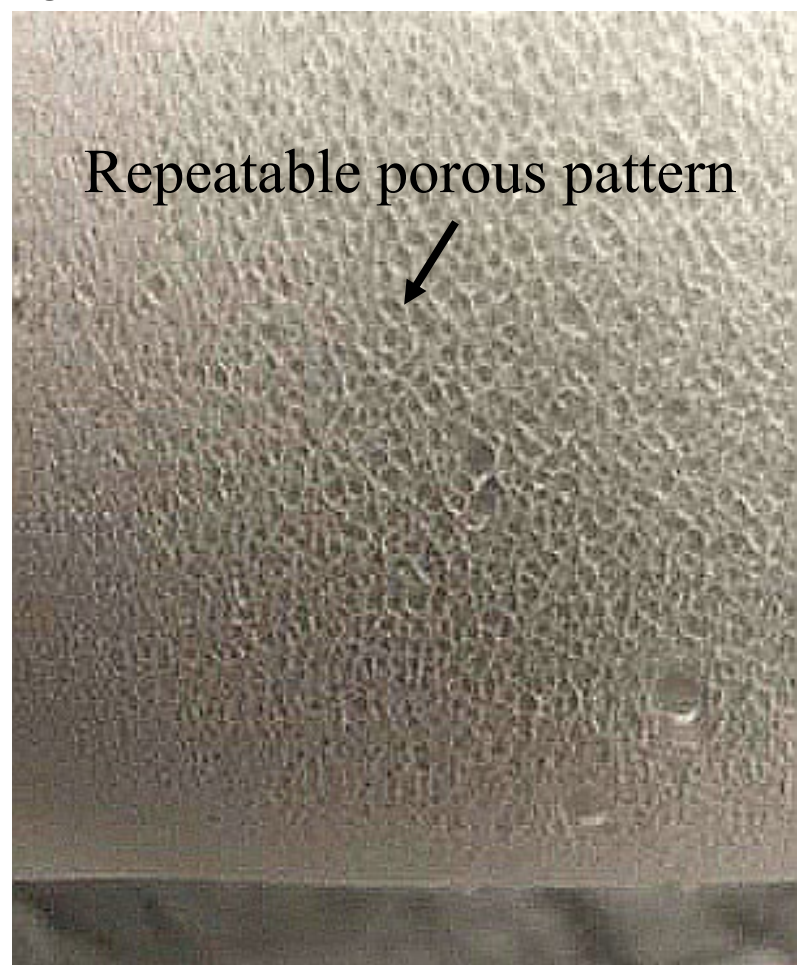

Figure S1. The morphology of the honeycomb scaffolds with ManCou-H after electrospinning on rotating collector as seen through a naked eye

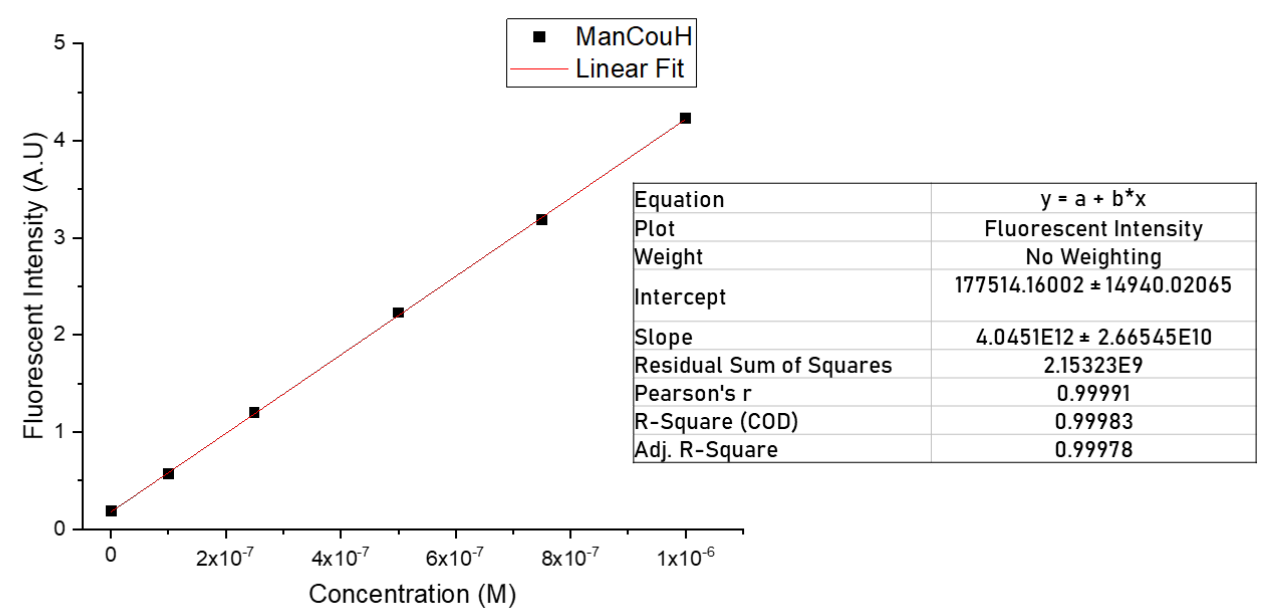


Figure S2. Calibration curve of the ManCou-H fluorescent probes in PBS.
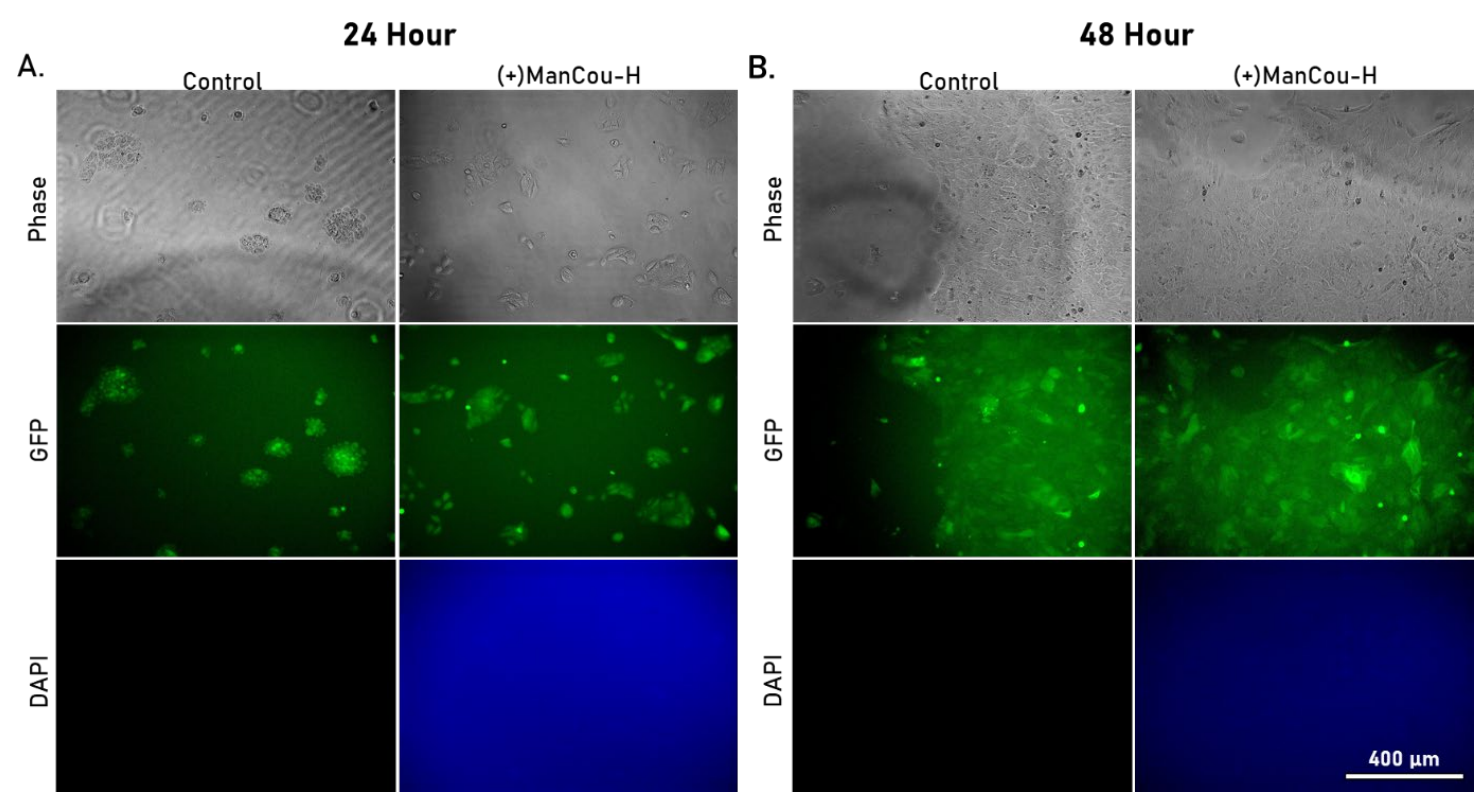

Figure S3. 184B5 cells stained with cell tracker green seeded on 2D culture plate imaged in Phase, GFP and DAPI (ManCou-H) (A) 24-hour time interval on control (no ManCou$\mathrm{H}$ ) and plates supplemented with $20 \mu \mathrm{M}$ ManCou-H (B) 48-hour time interval on control (no ManCou-H) and plates supplemented with $20 \mu \mathrm{M}$ ManCou-H. Images captured at 10x magnification. Images in DAPI on control were not taken. 


\section{Hour}

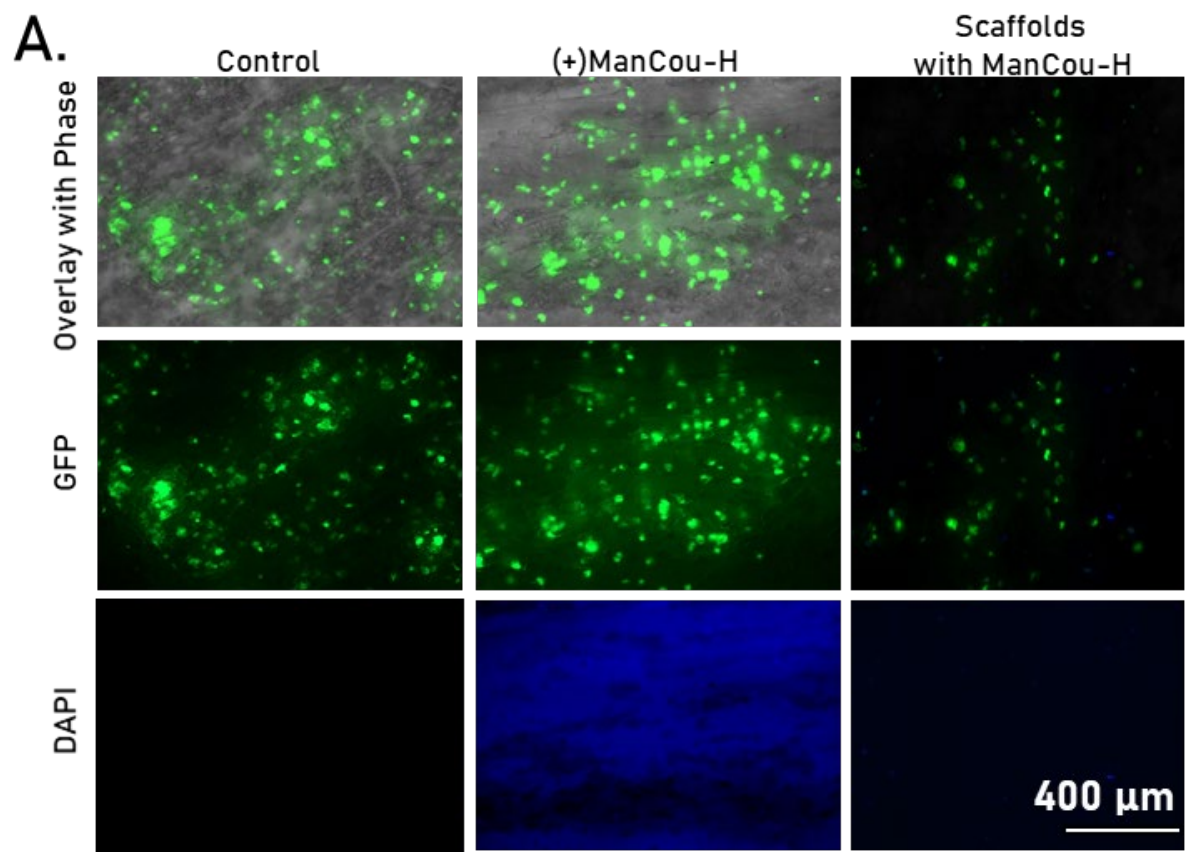

\section{Hour}

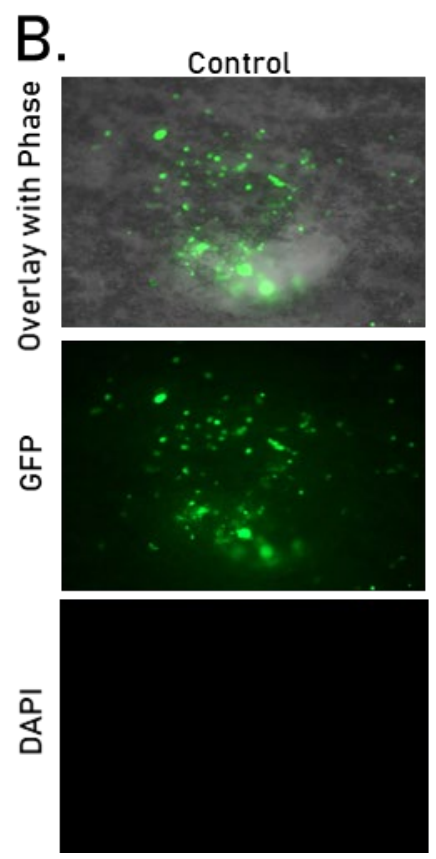

Scaffolds

(+)ManCou-H with ManCou-H
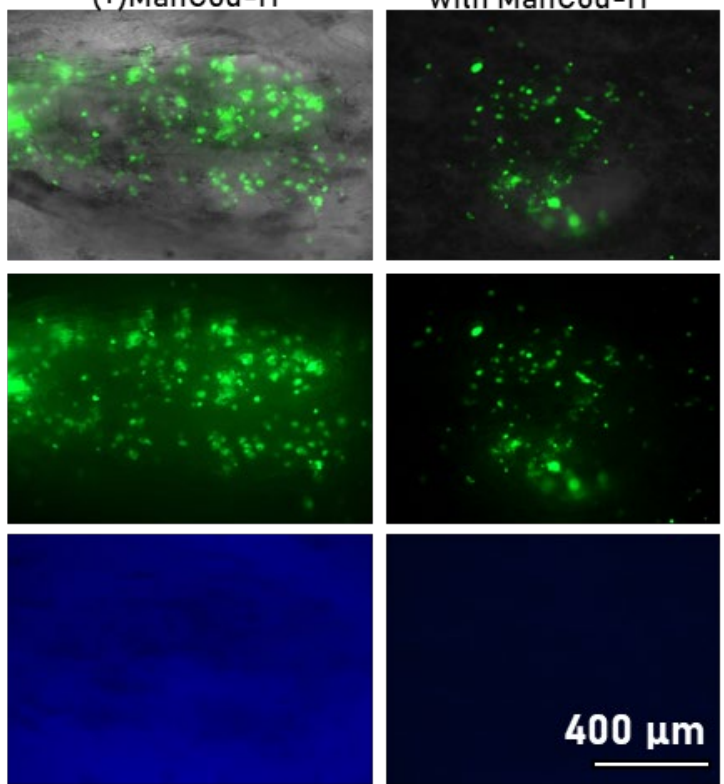
Figure S4. 184B5 cells in cell tracker green seeded on aligned scaffolds and imaged in phase, GFP, and DAPI. (A) 24 hours with and without $20 \mu \mathrm{M}$ ManCou-H media and scaffolds containing ManCou-H (B) 48 hours with and without $20 \mu \mathrm{M}$ ManCou-H media and scaffolds containing ManCou-H. Images captured at 10x magnification. DAPI images in conditions without ManCou-H were not taken.

\section{Hour}

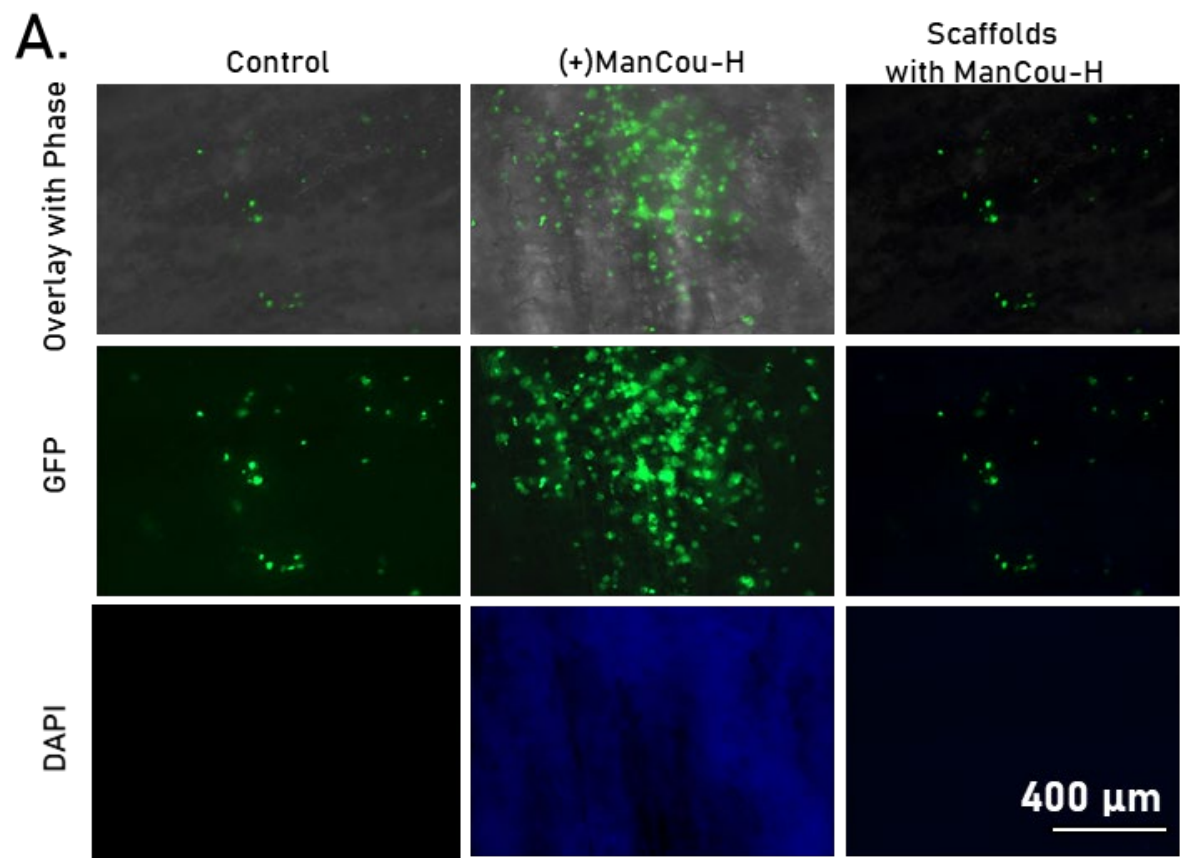




\section{Hour}

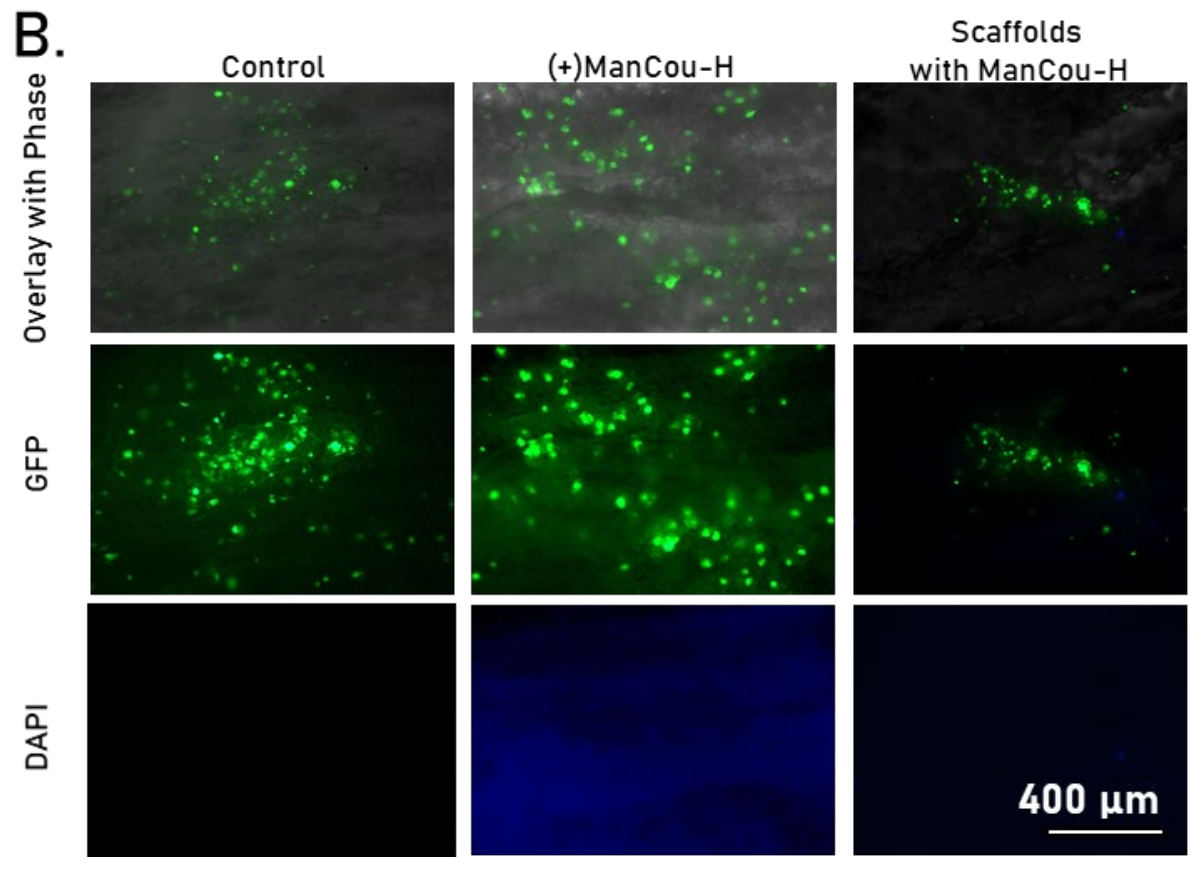

Figure S5. 184B5 cells in cell tracker green seeded on honeycomb scaffolds and imaged in phase, GFP, and DAPI. (A) 24 hours with and without $20 \mu \mathrm{M}$ ManCou-H media and scaffolds containing ManCou-H (B) 48 hours with and without $20 \mu \mathrm{M}$ ManCou-H media and scaffolds containing ManCou-H. Images captured at 10x magnification. DAPI images in conditions without ManCou-H were not taken. 


\section{Hour}

A.

Control

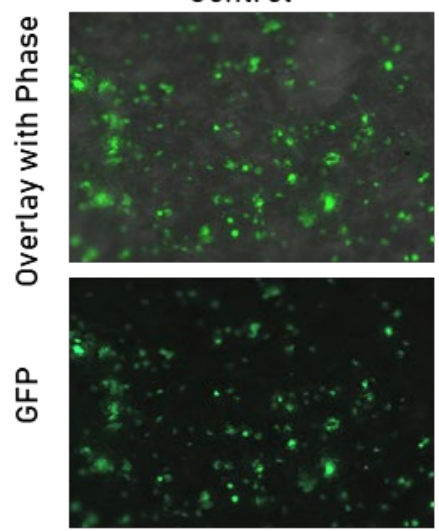

高

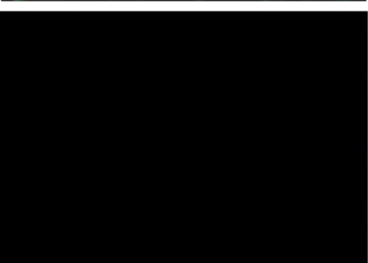

B.

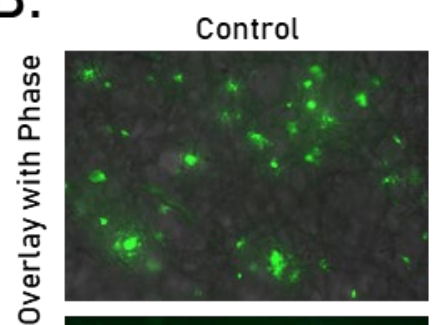

ठ

눙

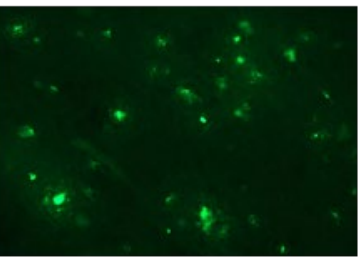

(+)ManCou-H
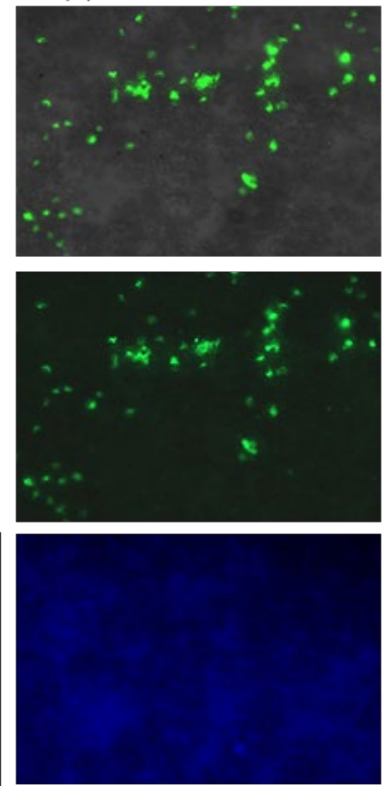

48 Hour

(+)ManCou-H
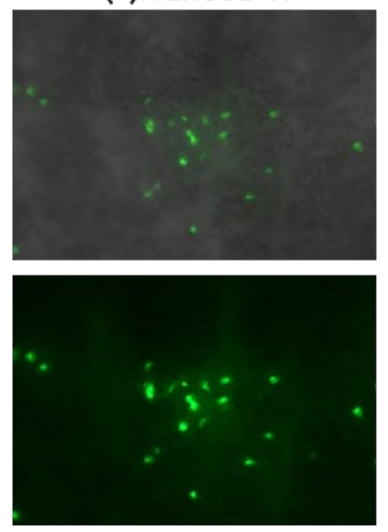

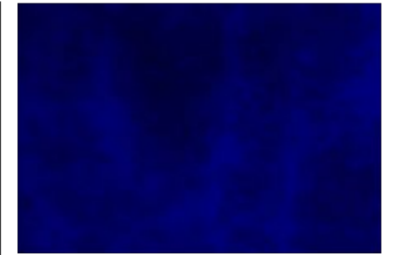

Scaffolds

with ManCou-H
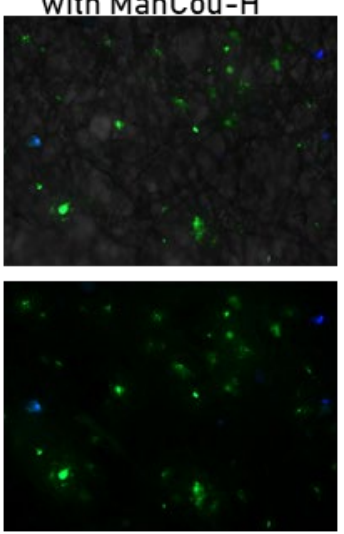

$400 \mu \mathrm{m}$ 
Figure S6. 184B5 cells in cell tracker green seeded on mesh scaffolds and imaged in phase, GFP, and DAPI. (A) 24 hours with and without $20 \mu \mathrm{M}$ ManCou-H media and scaffolds containing ManCou-H (B) 48 hours with and without $20 \mu \mathrm{M}$ ManCou-H media and scaffolds containing ManCou-H. Images captured at 10x magnification. DAPI images in conditions without ManCou-H were not taken.
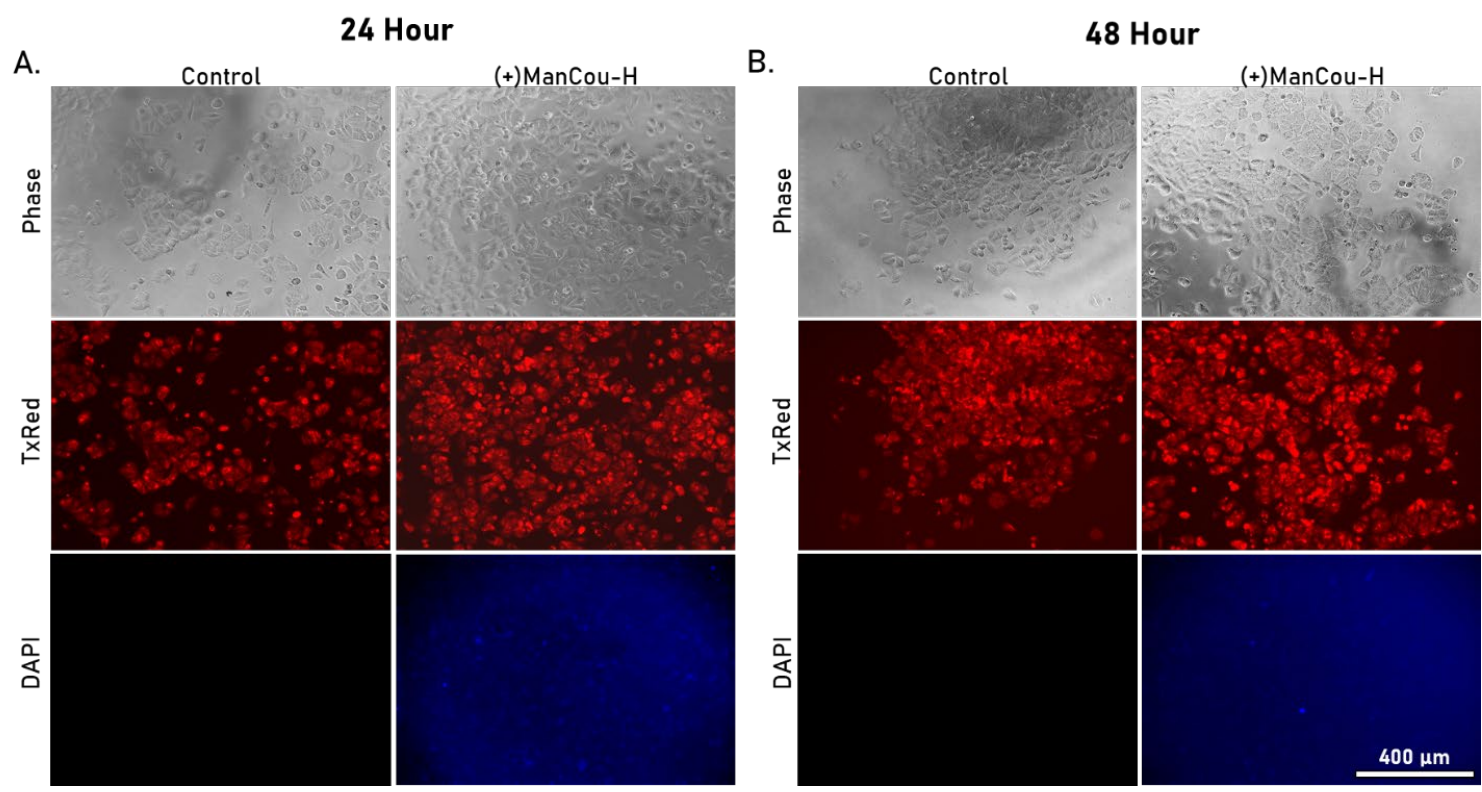

Figure S7. MCF7 cells stained with cell tracker red seeded on 2D culture plate imaged in Phase, TxRed and DAPI (ManCou-H) (A) 24-hour time interval on control (no ManCou$\mathrm{H}$ ) and plates supplemented with $20 \mu \mathrm{M}$ ManCou-H (B) 48-hour time interval on control (no ManCou-H) and plates supplemented with $20 \mu \mathrm{M}$ ManCou-H. Images captured at 10x magnification. Images in DAPI on control were not taken. 

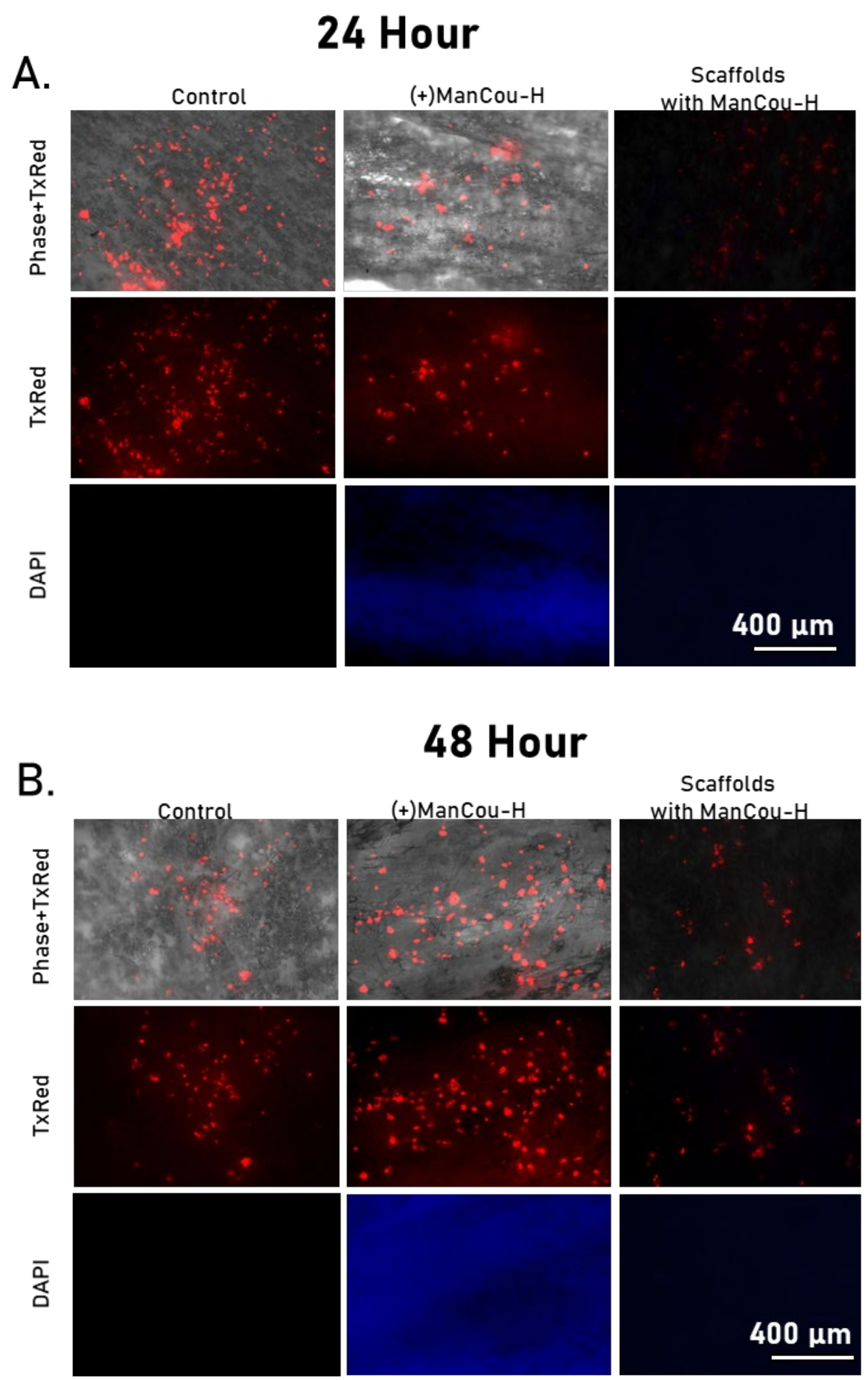

Figure S8. MCF7 cells in cell tracker red seeded on aligned scaffolds and imaged in phase, TxRed, and DAPI. (A) 24 hours with and without $20 \mu \mathrm{M}$ ManCou-H media and scaffolds containing ManCou-H (B) 48 hours with and without $20 \mu \mathrm{M}$ ManCou-H media and 
scaffolds containing ManCou-H. Images captured at 10x magnification. DAPI images in conditions without ManCou-H were not taken.

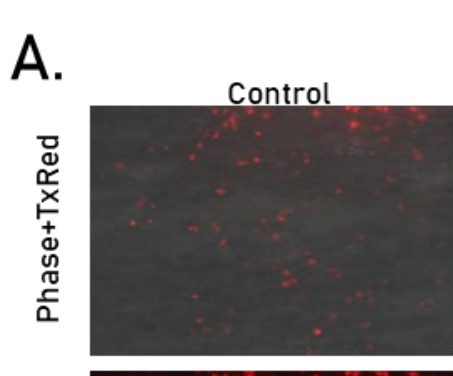

\section{Hour}
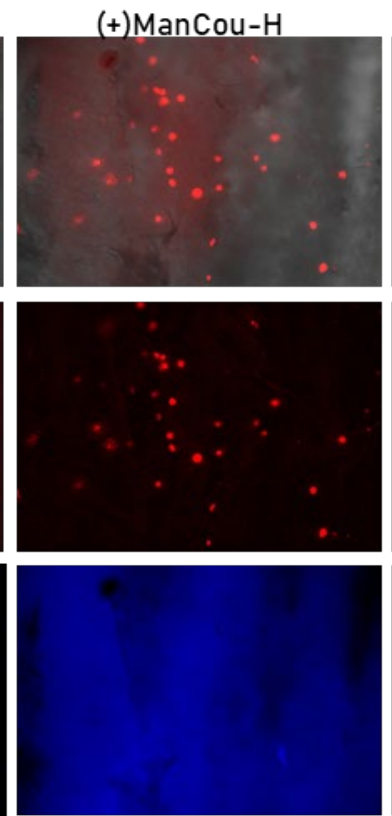

Scaffolds

with ManCou-H
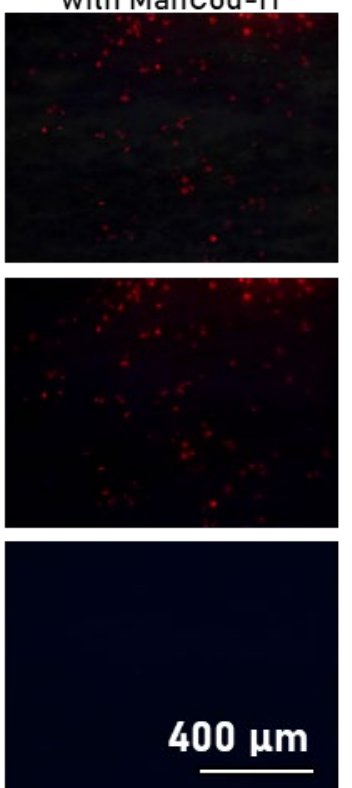


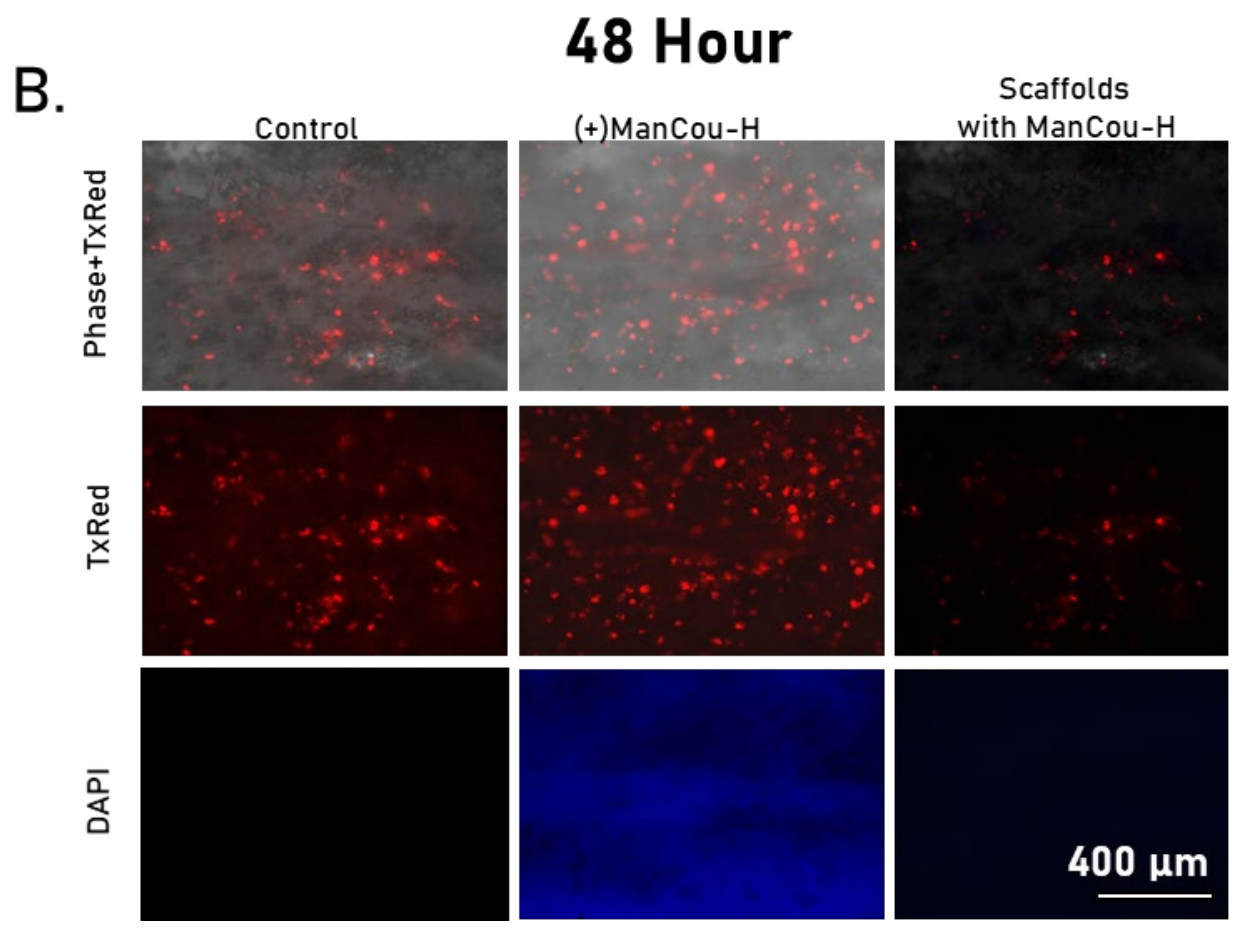

Figure S9. MCF7 cells in cell tracker red seeded on honeycomb scaffolds and imaged in phase, TxRed, and DAPI. (A) 24 hours with and without $20 \mu \mathrm{M}$ ManCou-H media and scaffolds containing ManCou-H (B) 48 hours with and without $20 \mu \mathrm{M}$ ManCou-H media and scaffolds containing ManCou-H. Images captured at 10x magnification. DAPI images in conditions without ManCou-H were not taken. 

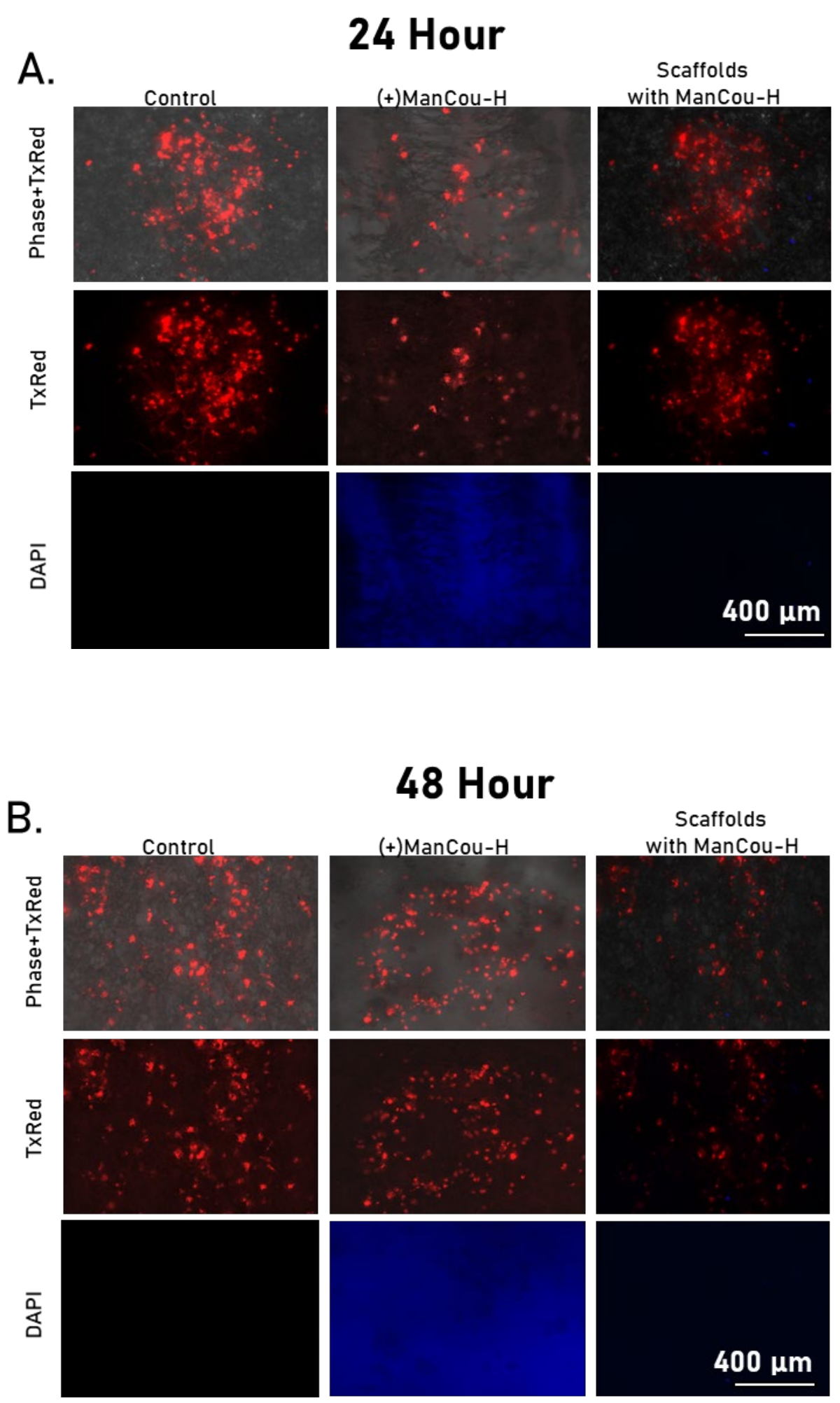

Figure S10. MCF7 cells in cell tracker red seeded on mesh scaffolds and imaged in phase, TxRed, and DAPI. (A) 24 hours with and without $20 \mu \mathrm{M}$ ManCou-H media and scaffolds 
containing ManCou-H (B) 48 hours with and without $20 \mu \mathrm{M}$ ManCou-H media and scaffolds containing ManCou-H. Images captured at 10x magnification. DAPI images in conditions without ManCou-H were not taken.
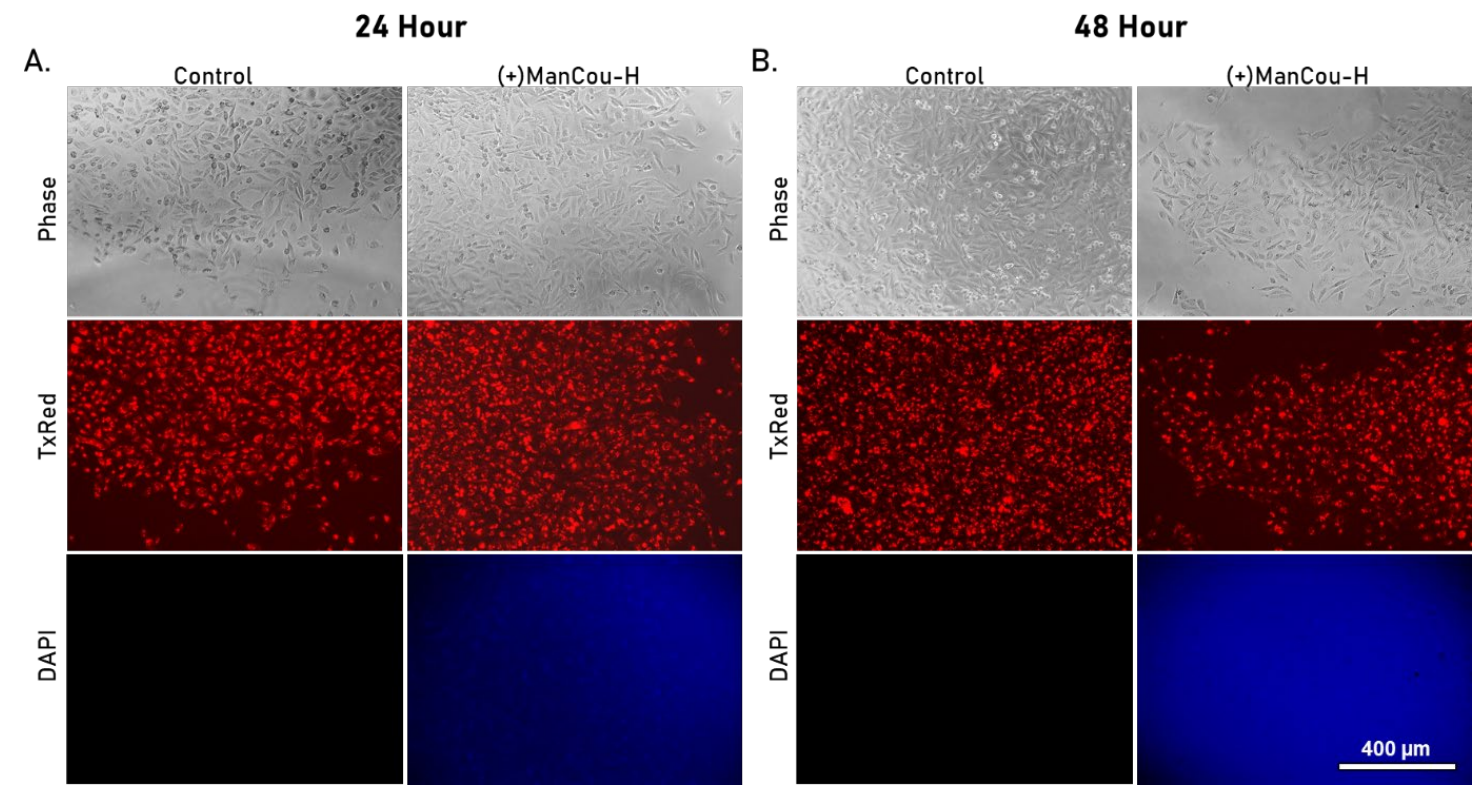

Figure S11. MDA-MB-231 cells stained with cell tracker red seeded on 2D culture plate imaged in Phase, TxRed and DAPI (ManCou-H) (A) 24-hour time interval on control (no ManCou-H) and plates supplemented with $20 \mu \mathrm{M}$ ManCou-H (B) 48-hour time interval on control (no ManCou-H) and plates supplemented with $20 \mu \mathrm{M}$ ManCou-H. Images captured at 10x magnification. Images in DAPI on control were not taken. 

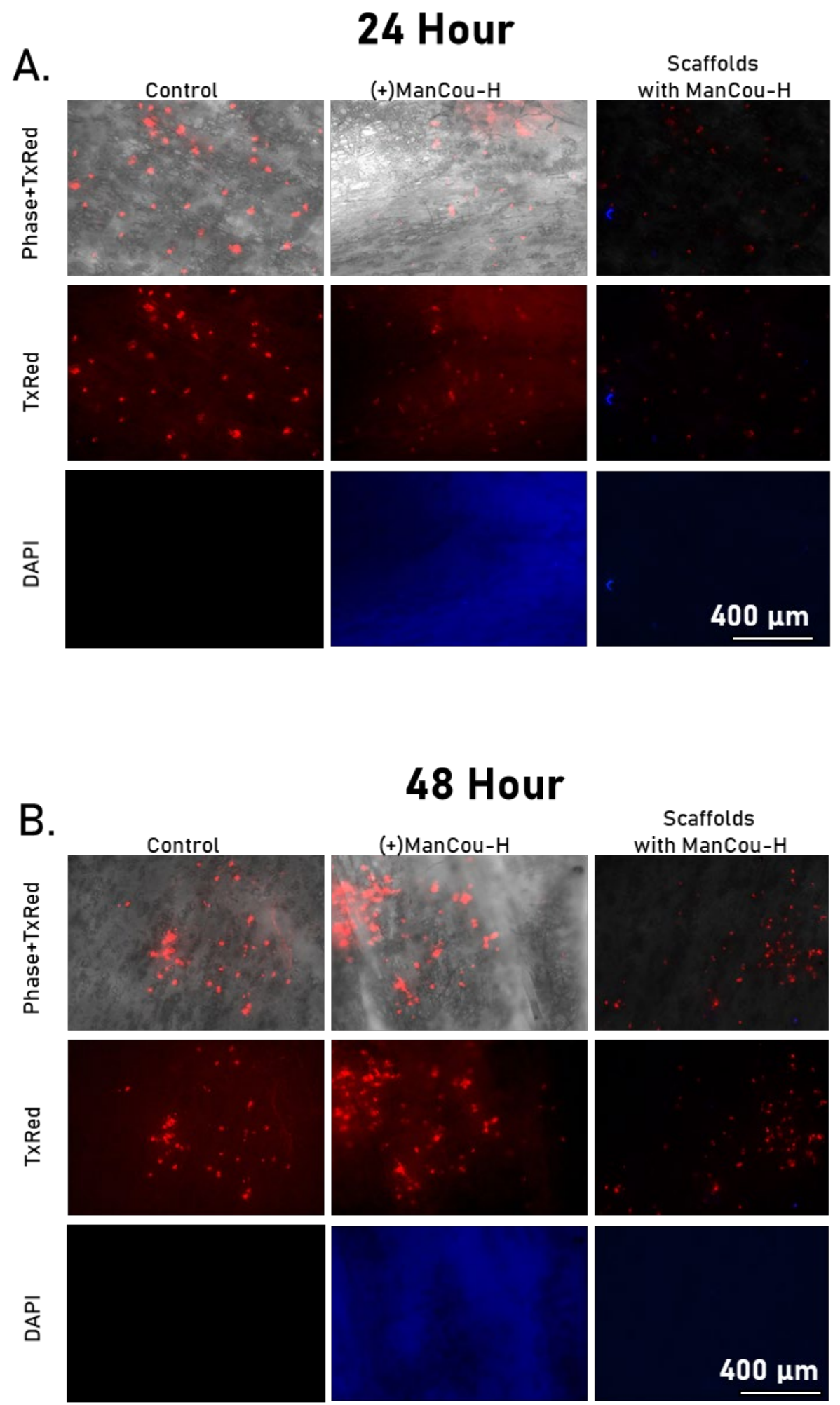
Figure S12. MDA-MB-231 cells in cell tracker red seeded on aligned scaffolds and imaged in phase, TxRed, and DAPI. (A) 24 hours with and without $20 \mu \mathrm{M}$ ManCou-H media and scaffolds containing ManCou-H (B) 48 hours with and without $20 \mu \mathrm{M}$ ManCou-H media and scaffolds containing ManCou-H. Images captured at 10x magnification. DAPI images in conditions without ManCou-H were not taken.

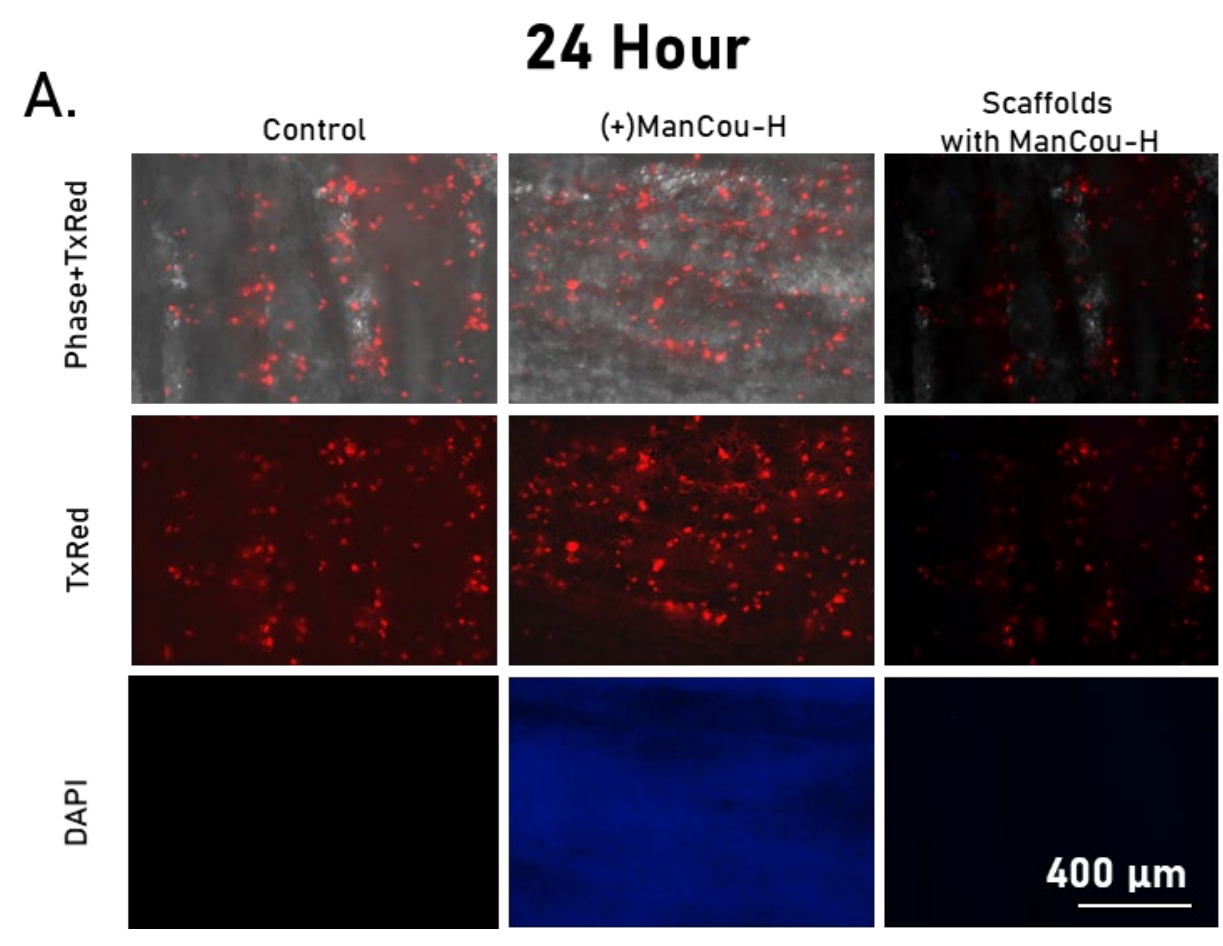




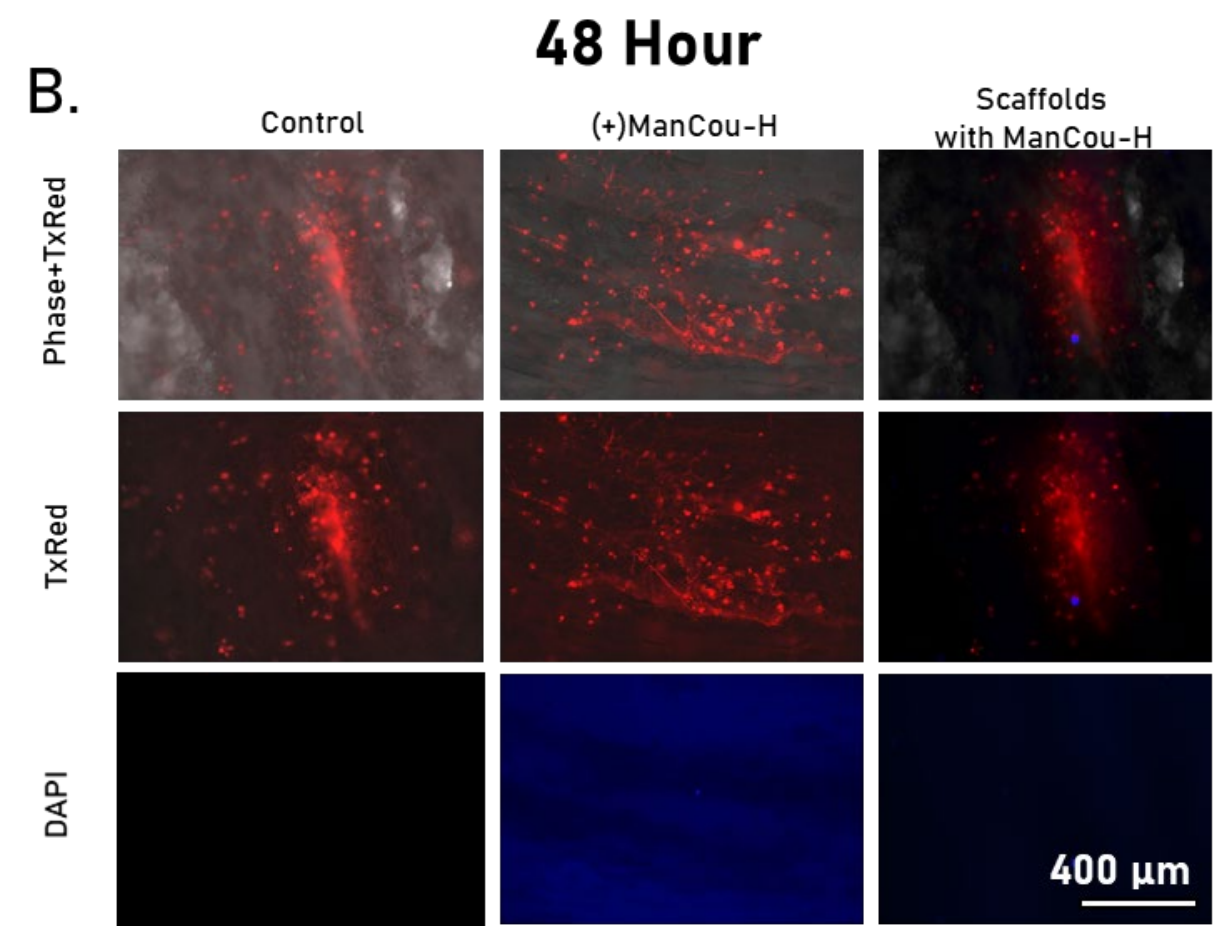

Figure S13. MDA-MB-231 cells in cell tracker red seeded on honeycomb scaffolds and imaged in phase, TxRed, and DAPI. (A) 24-hour with and without $20 \mu \mathrm{M}$ ManCou-H media and scaffolds containing ManCou-H (B) 48-hour with and without $20 \mu \mathrm{M}$ ManCou-H media and scaffolds containing ManCou-H. Images captured at 10x magnification. DAPI images in conditions without ManCou-H were not taken. 


\section{Hour}

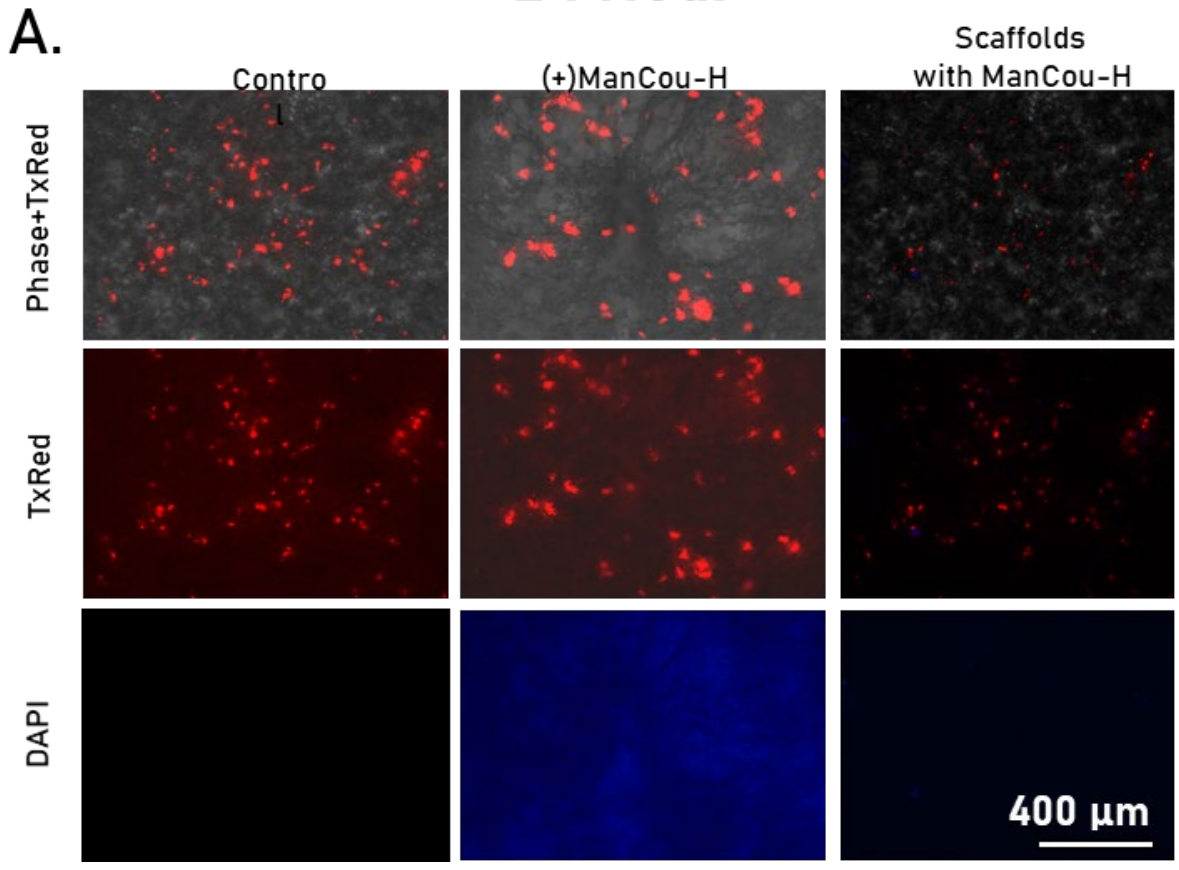

\section{Hour}

B.

(+)ManCou-H

Scaffolds
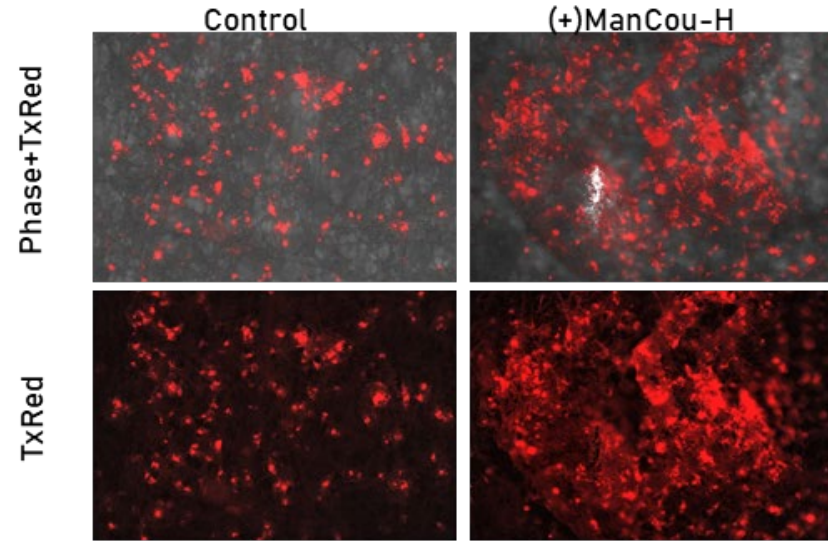

with ManCou-H
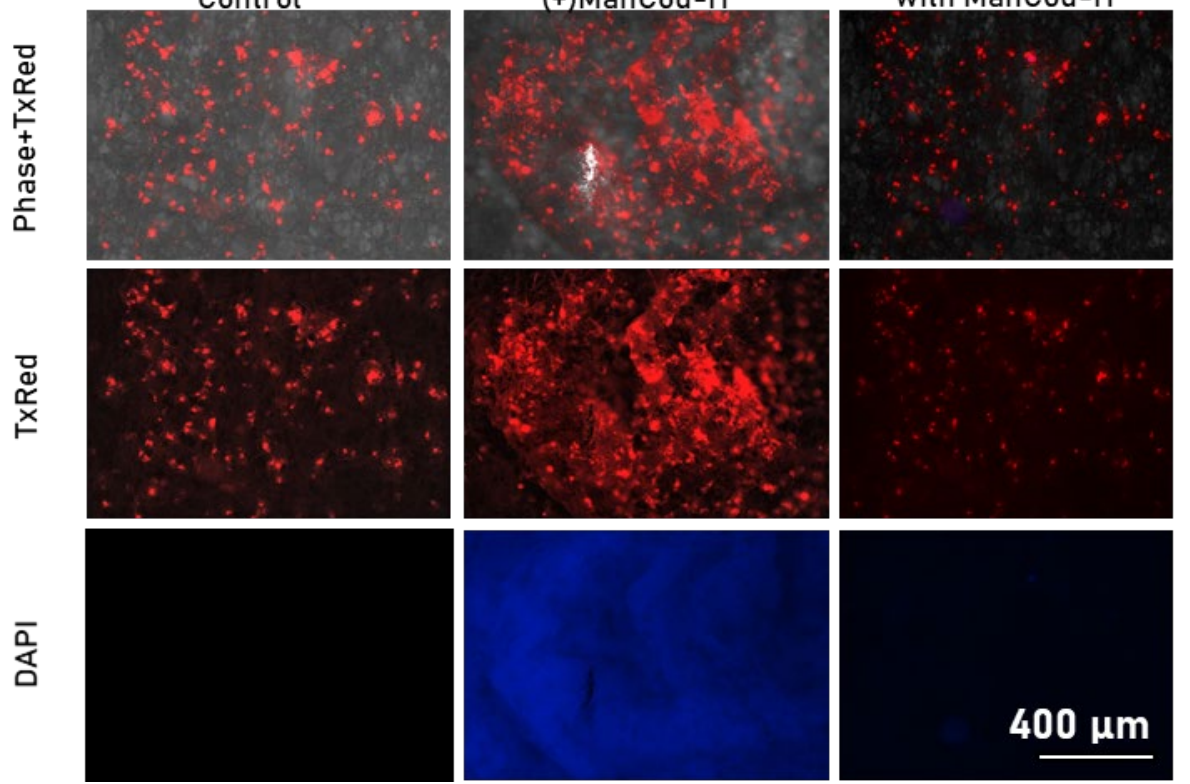
Figure S14. MDA-MB-231 cells in cell tracker red seeded on mesh scaffolds and imaged in phase, TxRed, and DAPI. (A) 24 hours with and without $20 \mu \mathrm{M}$ ManCou-H media and scaffolds containing ManCou-H (B) 48 hours with and without $20 \mu \mathrm{M}$ ManCou-H media and scaffolds containing ManCou-H. Images captured at 10x magnification. DAPI images in conditions without ManCou-H were not taken.
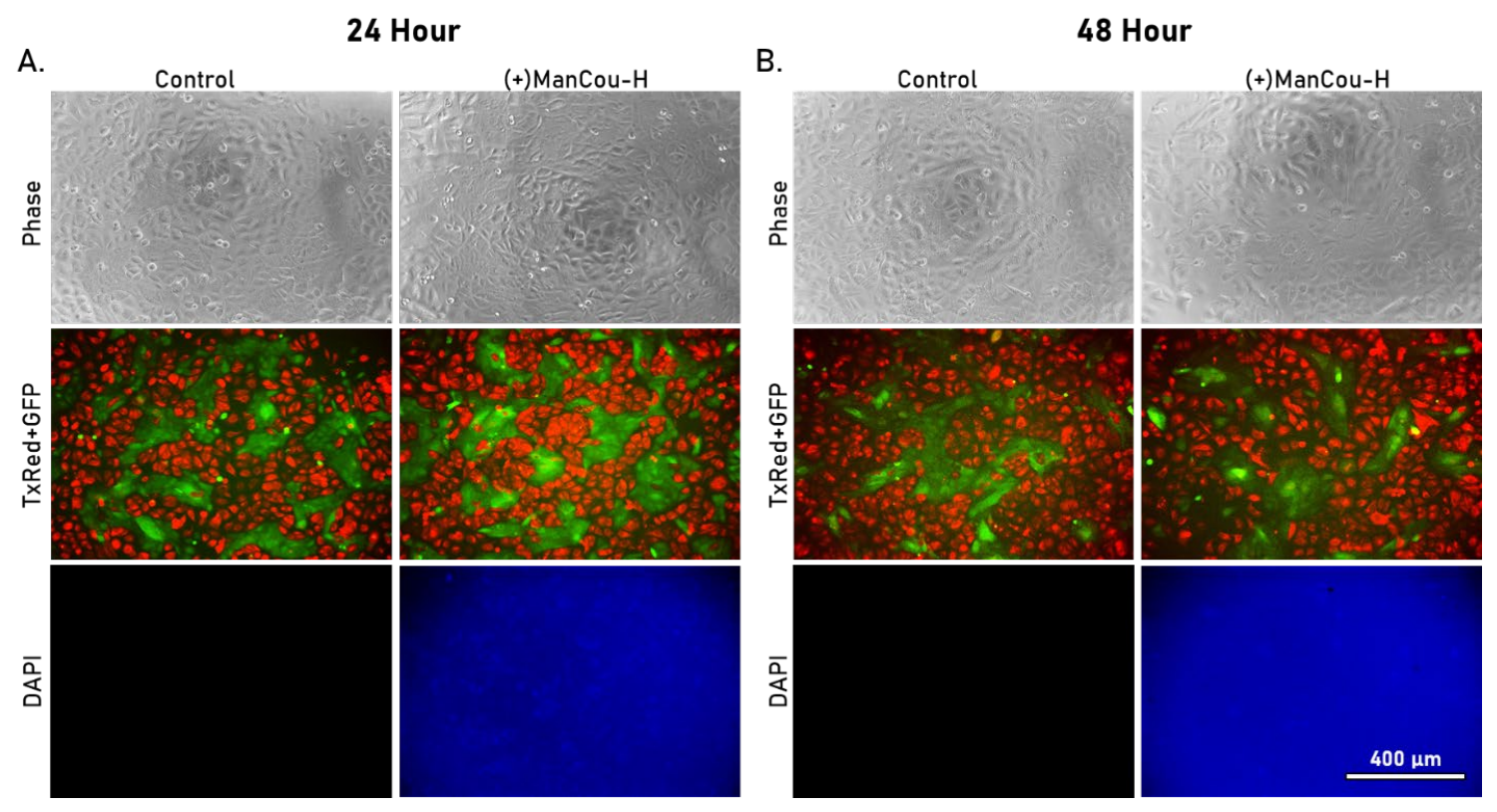

Figure S15. 184B5 cells stained with cell tracker green and MCF7 cells stained with cell tracker red seeded on 2D culture plate imaged in Phase, GFP and DAPI (ManCou-H) (A) 24-hour time interval on control (no ManCou-H) and plates supplemented with $20 \mu \mathrm{M}$ ManCou-H (B) 48-hour time interval on control (no ManCou-H) and plates supplemented with $20 \mu \mathrm{M}$ ManCou-H. Images captured at 10x magnification. Images in DAPI on control were not taken. 

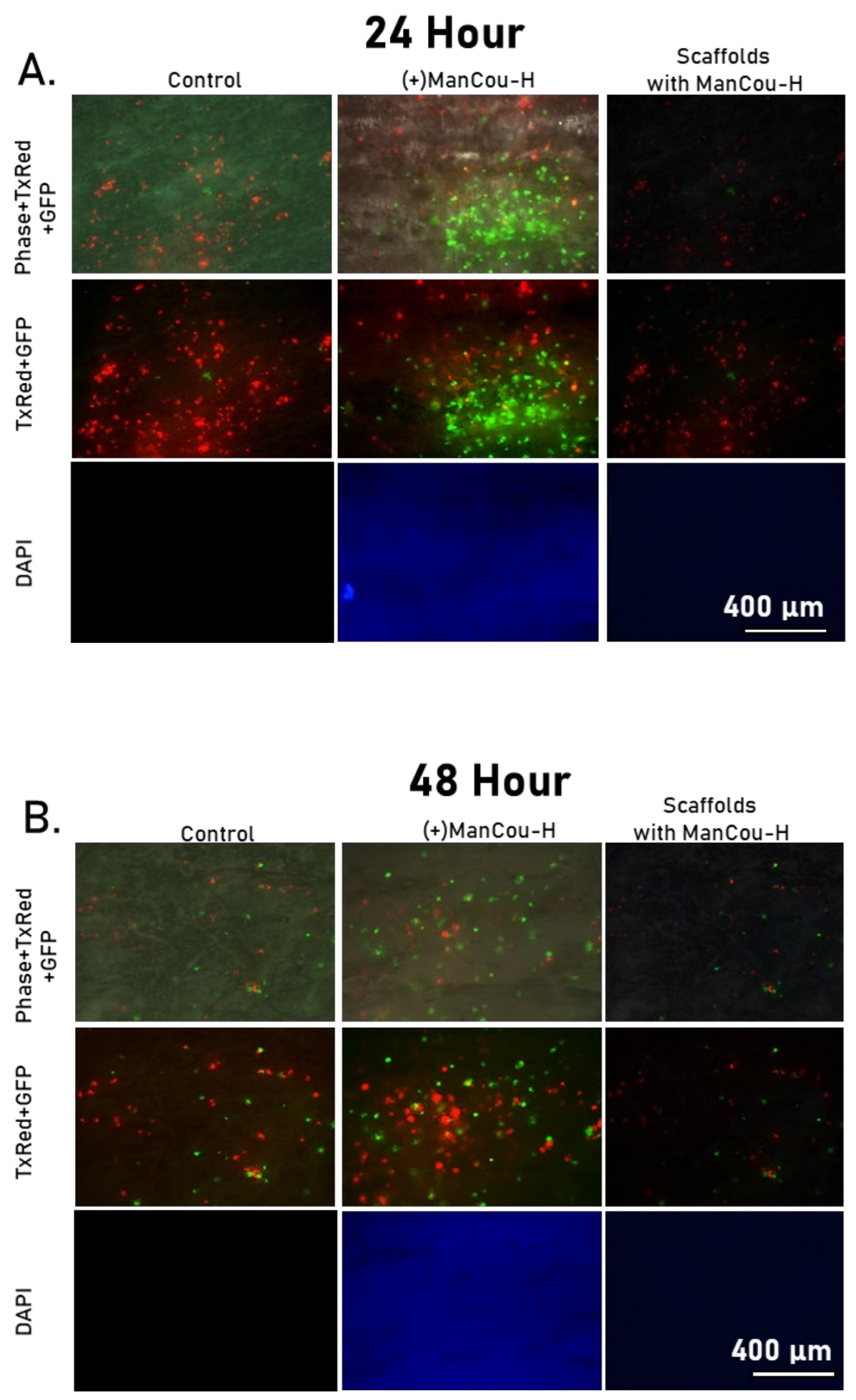
Figure S16. 184B5 cells in cell tracker green and MCF7 cells in cell tracker red seeded in coculture on aligned scaffolds and imaged in phase, TxRed and DAPI (A) 24 hours with and without $20 \mu \mathrm{M}$ ManCou-H media and scaffolds containing ManCou-H (B) 48 hours with and without $20 \mu \mathrm{M}$ ManCou-H media and scaffolds containing ManCou-H. Images captured at 10x magnification. DAPI images in conditions without ManCou-H were not taken.

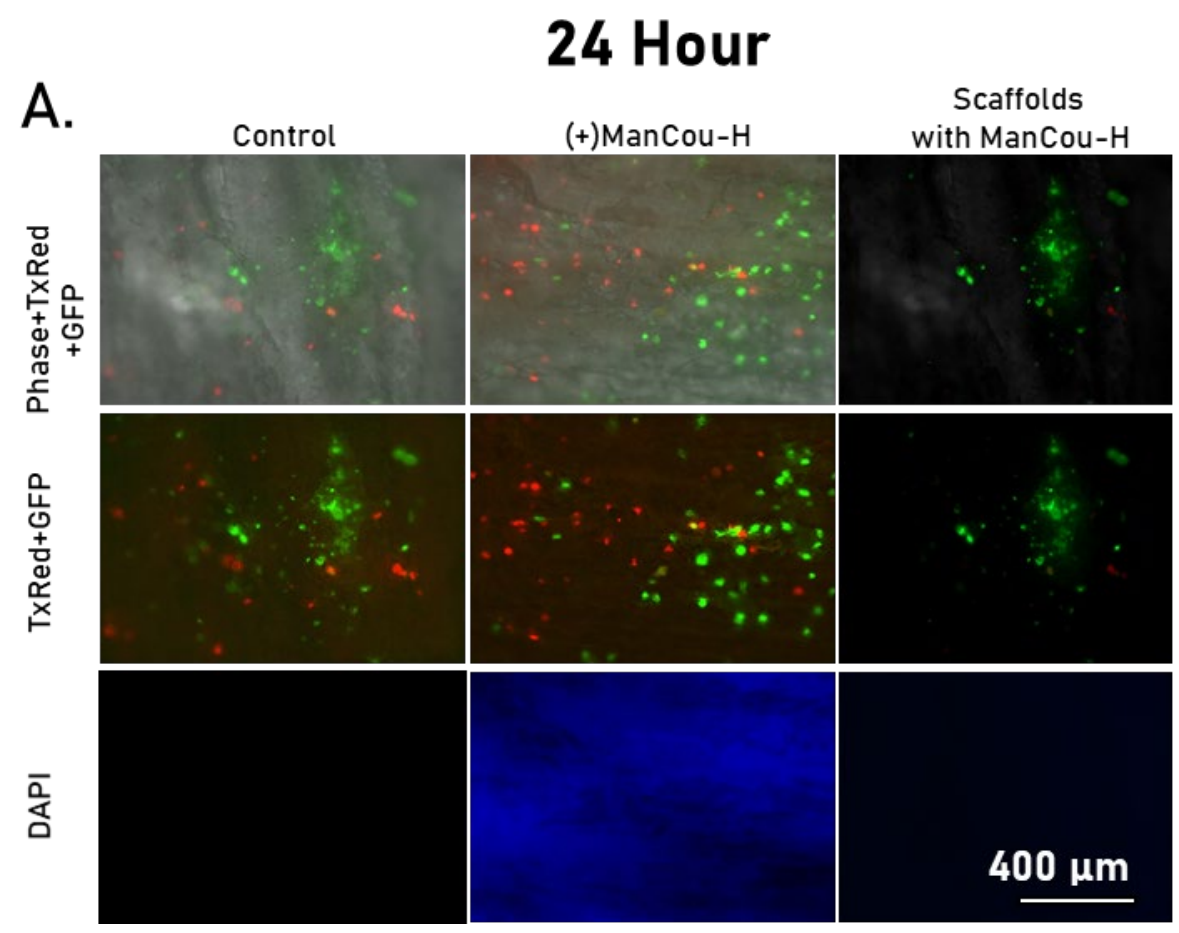




\section{Hour}

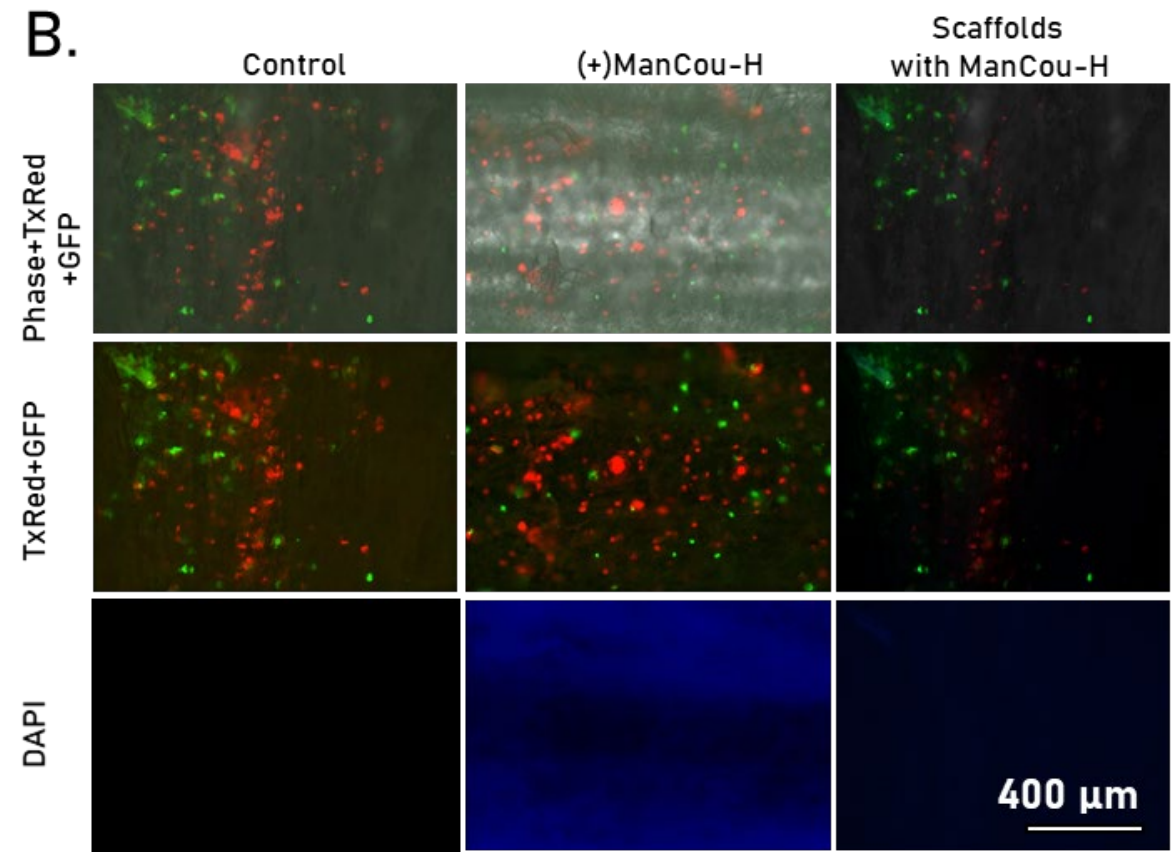

Figure S17. 184B5 cells in cell tracker green and MCF7 cells in cell tracker red seeded in coculture on honeycomb scaffolds and imaged in phase, TxRed and DAPI (A) 24 hours with and without $20 \mu \mathrm{M}$ ManCou-H media and scaffolds containing ManCou-H (B) 48 hours with and without $20 \mu \mathrm{M}$ ManCou-H media and scaffolds containing ManCou-H. Images captured at 10x magnification. DAPI images in conditions without ManCou-H were not taken. 


\section{Hour}

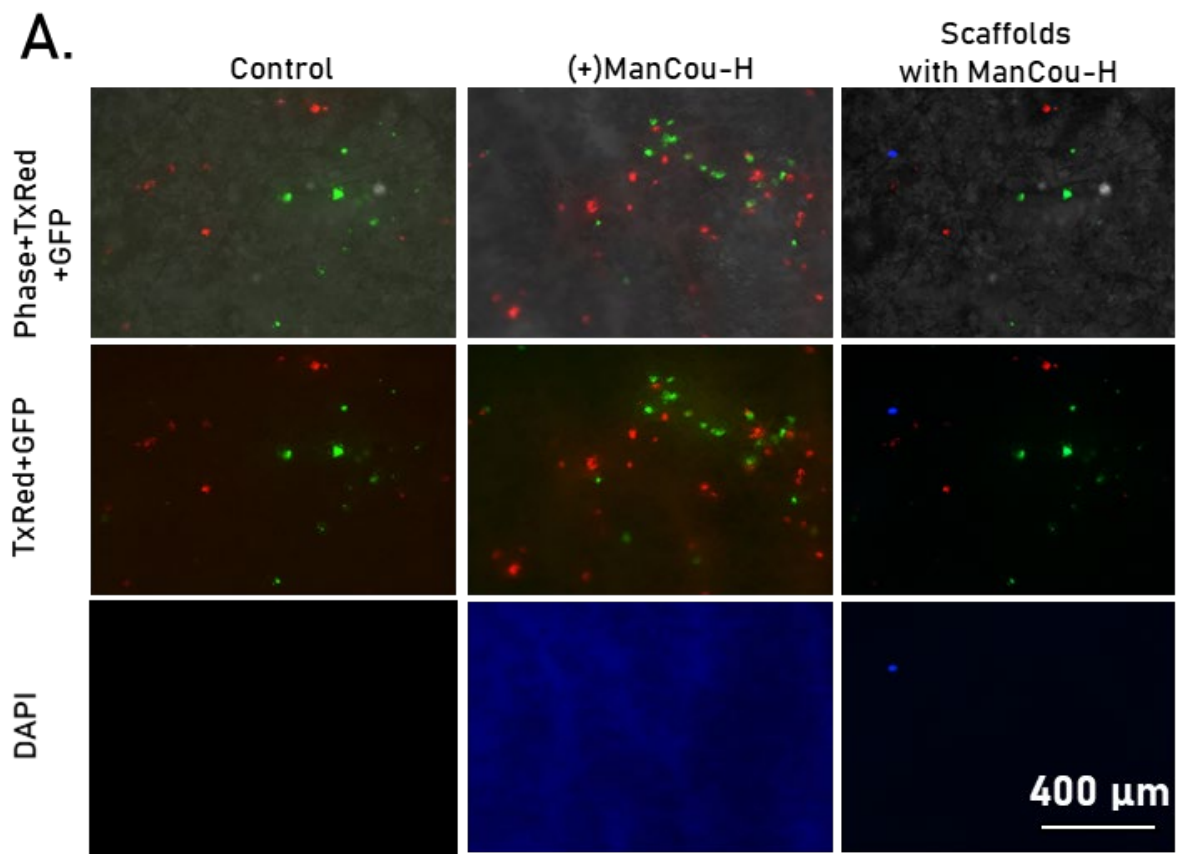

\section{Hour}

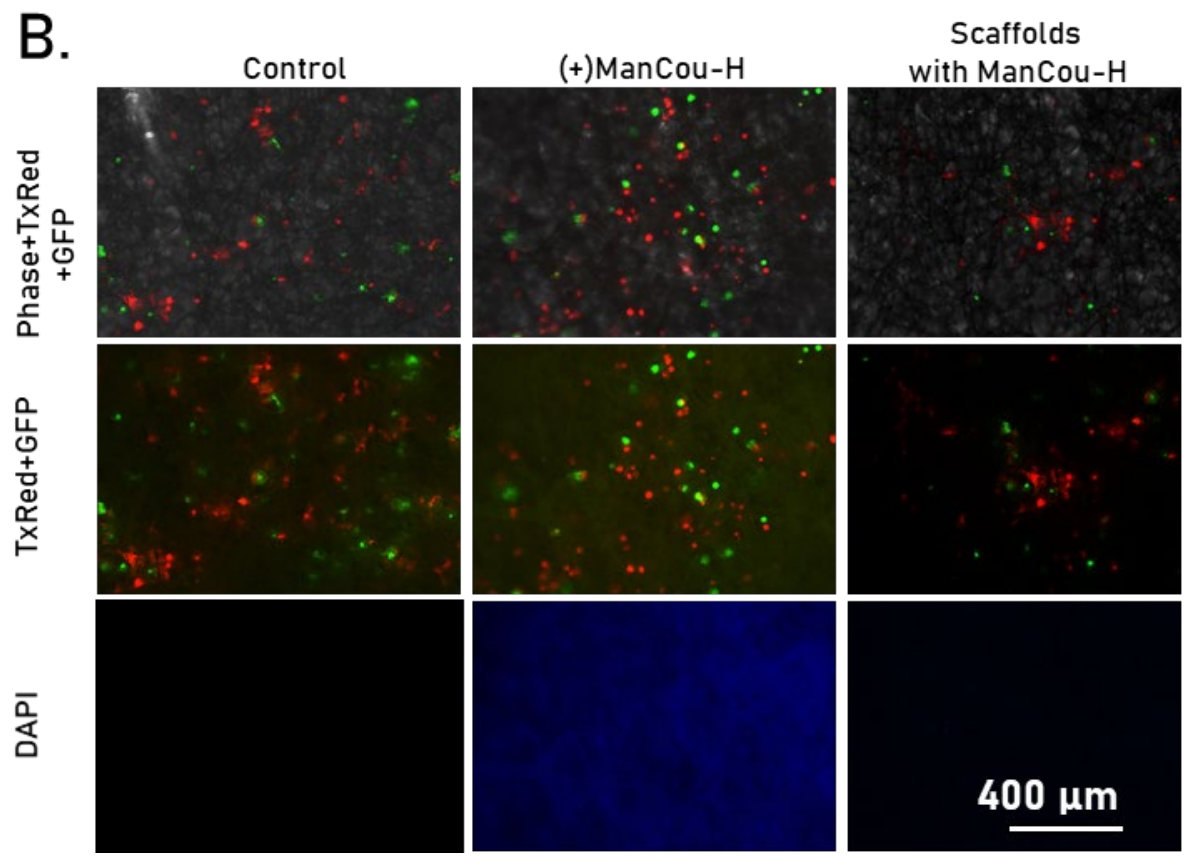

Figure S18. 184B5 cells in cell tracker green and MCF7 cells in cell tracker red seeded in coculture on mesh scaffolds and imaged in phase, TxRed and DAPI (A) 24 hours with and 
without $20 \mu \mathrm{M}$ ManCou-H media and scaffolds containing ManCou-H (B) 48 hours with and without $20 \mu \mathrm{M}$ ManCou-H media and scaffolds containing ManCou-H. Images captured at 10x magnification. DAPI images in conditions without $\mathrm{ManCou}-\mathrm{H}$ were not taken.
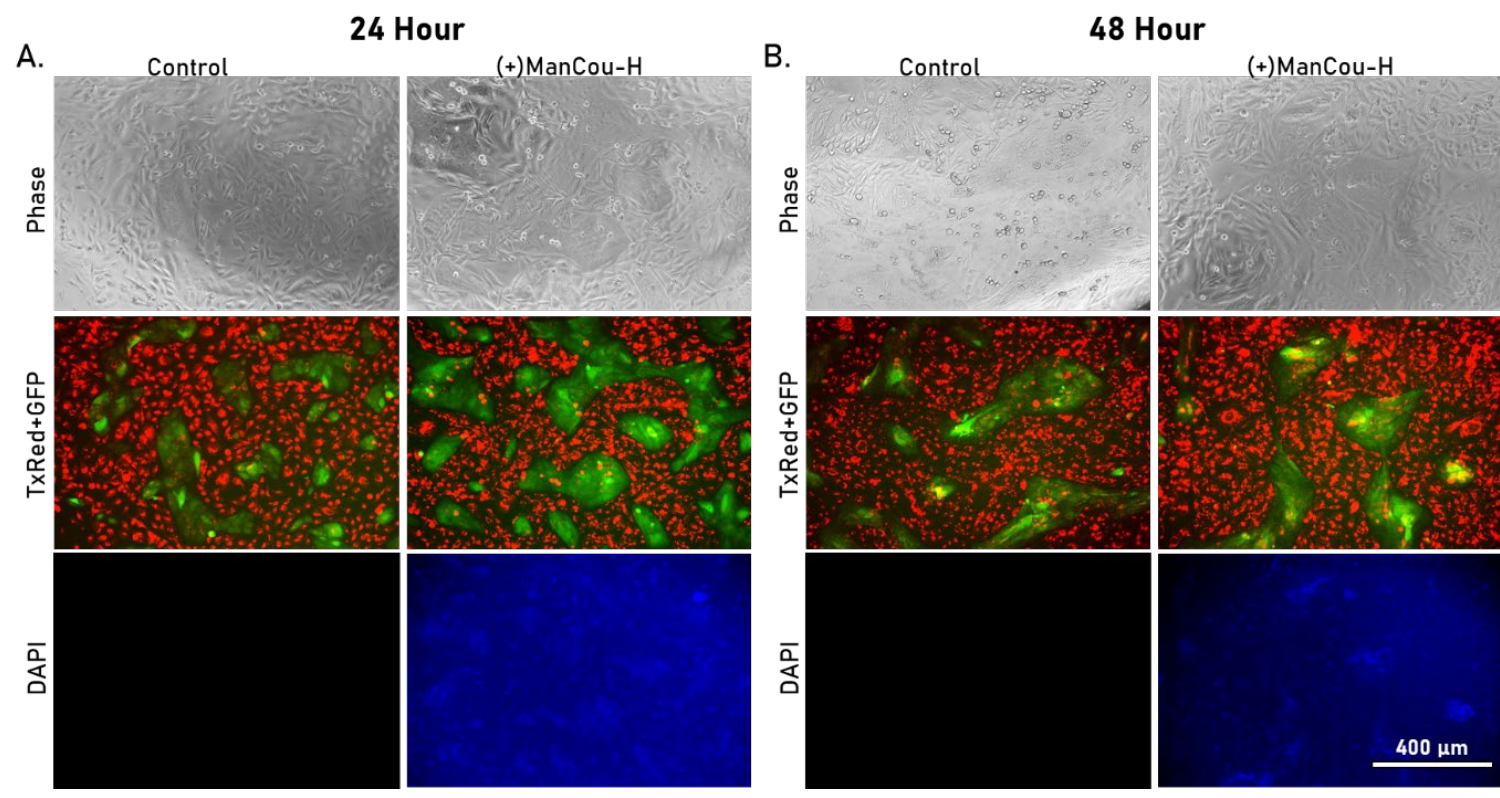

Figure S19. 184B5 cells stained with cell tracker green and MDA-MB-231 cells stained with cell tracker red seeded in coculture on 2D culture plate imaged in phase, TxRed and DAPI (A) 24-hour time interval on control (no ManCou-H) and plates supplemented with $20 \mu \mathrm{M}$ ManCou-H (B) 48-hour time interval on control (no ManCou-H) and plates supplemented with $20 \mu \mathrm{M}$ ManCou-H. Images captured at 10x magnification. Images in DAPI on control were not taken. 

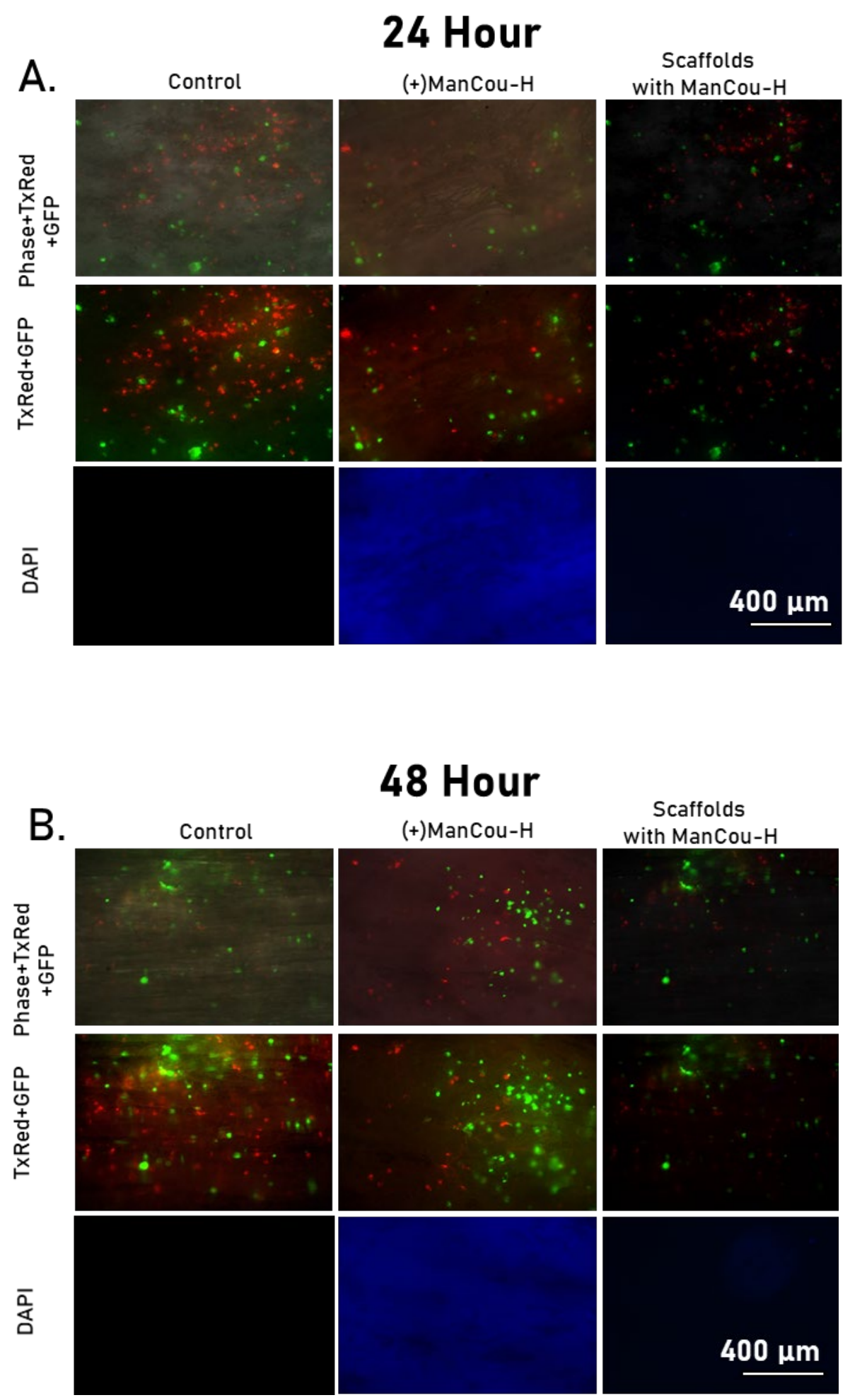

Figure S20. 184B5 cells in cell tracker green and MDA-MB-231 cells in cell tracker red seeded in coculture on aligned scaffolds and imaged in phase, TxRed and DAPI (A) 24 
hours with and without $20 \mu \mathrm{M}$ ManCou-H media and scaffolds containing ManCou-H (B) 48 hours with and without $20 \mu \mathrm{M}$ ManCou-H media and scaffolds containing ManCou$\mathrm{H}$. Images captured at 10x magnification. DAPI images in conditions without ManCou-H were not taken.

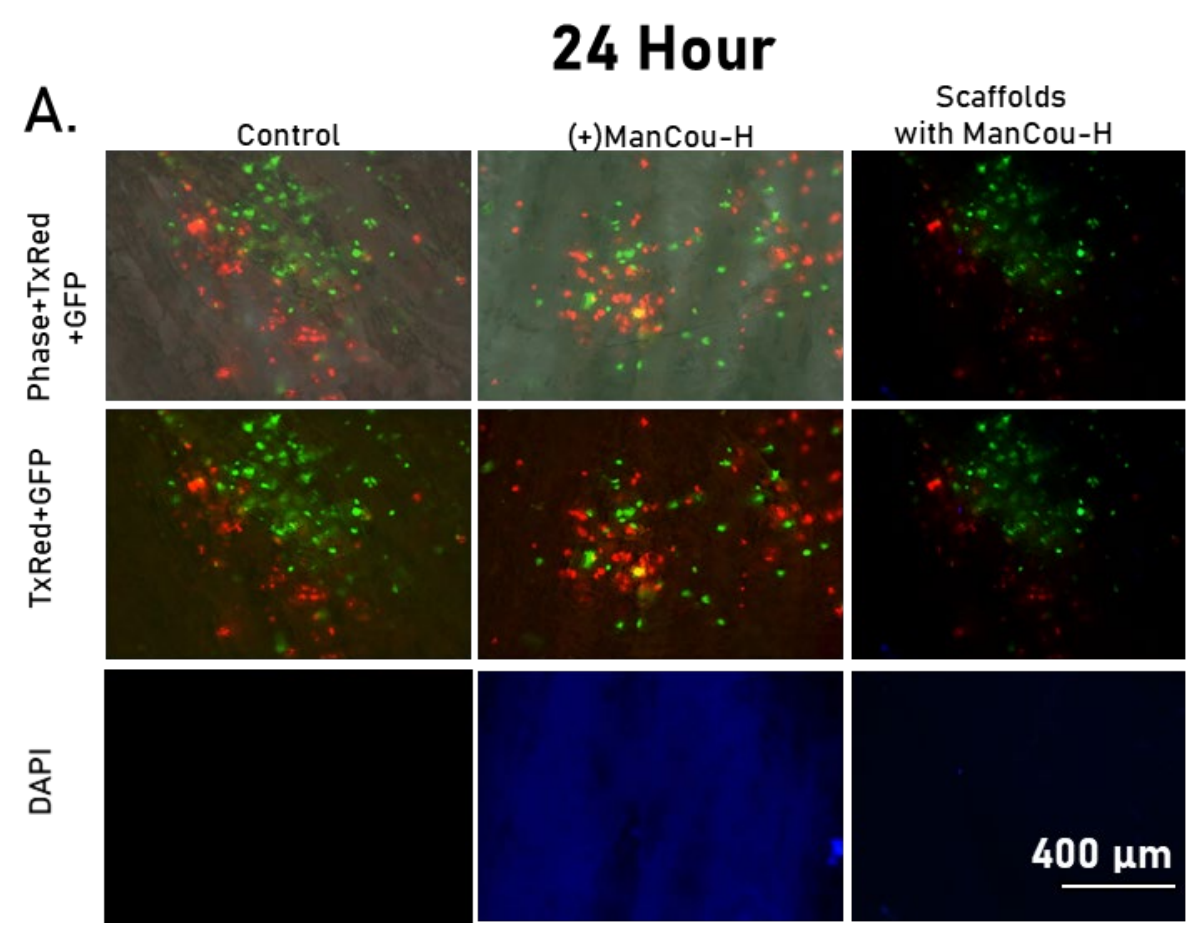




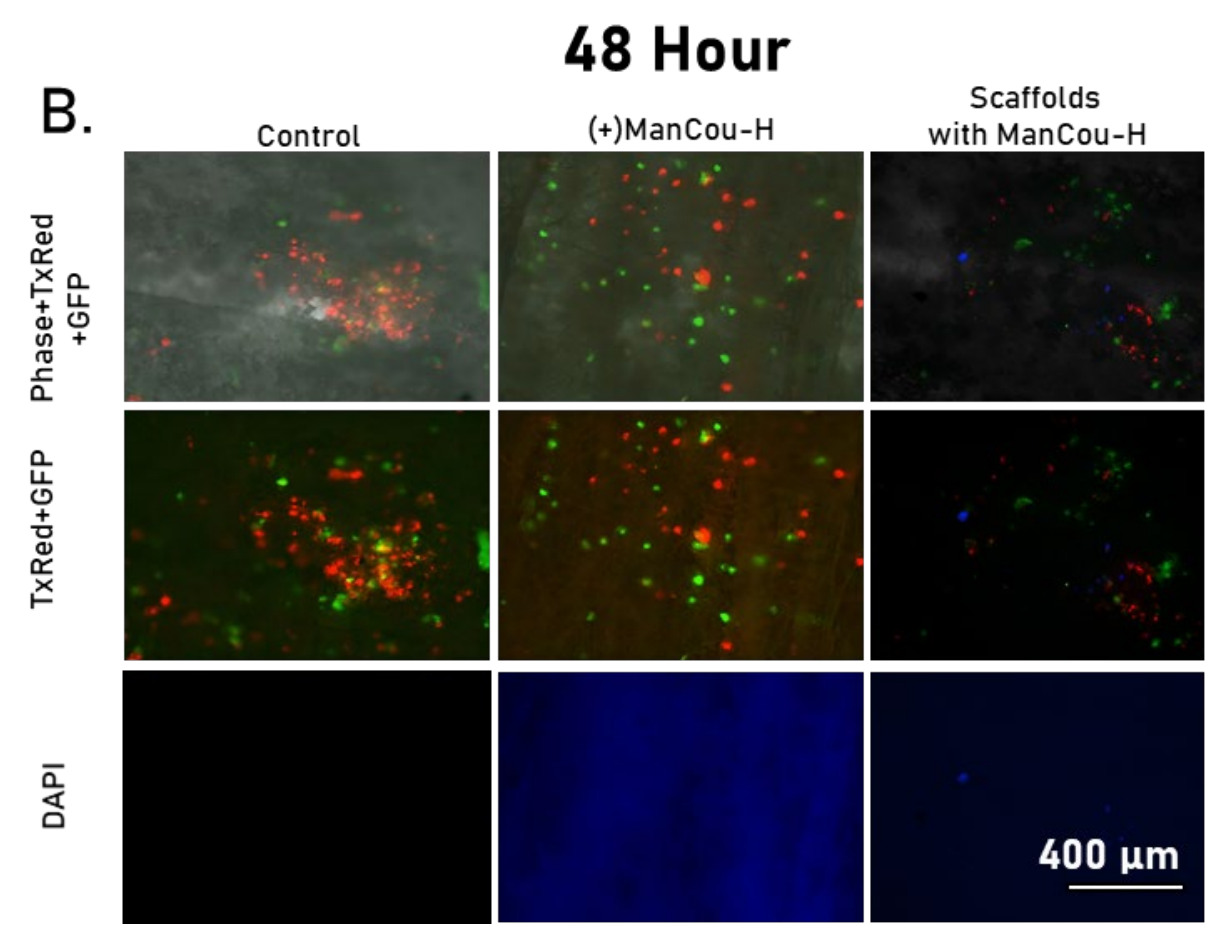

Figure S21. 184B5 cells in cell tracker green and MDA-MB-231 cells in cell tracker red seeded in coculture on honeycomb scaffolds and imaged in phase, TxRed and DAPI (A) 24 hours with and without $20 \mu \mathrm{M}$ ManCou-H media and scaffolds containing ManCou-H (B) 48 hours with and without $20 \mu \mathrm{M}$ ManCou-H media and scaffolds containing ManCou$\mathrm{H}$. Images captured at 10x magnification. DAPI images in conditions without ManCou-H were not taken. 


\section{Hour}

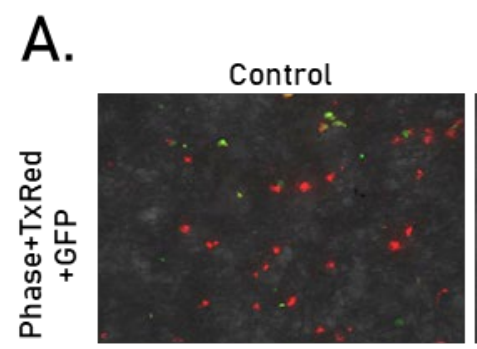

$(+)$ ManCou-H

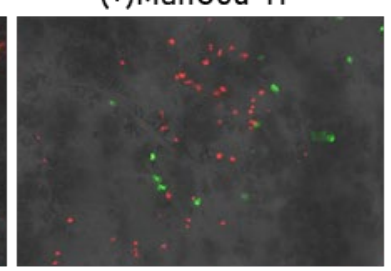

Scaffolds

with ManCou-H
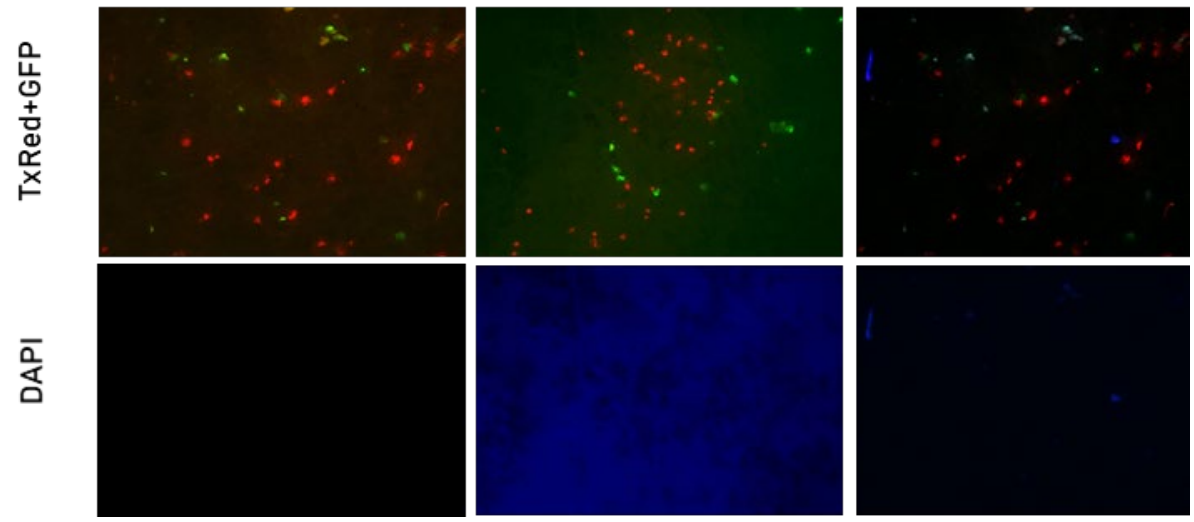

\section{Hour}

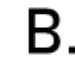

Control
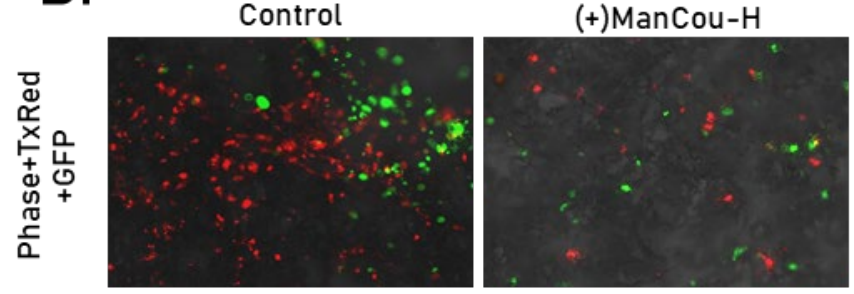

Scaffolds

with ManCou-H
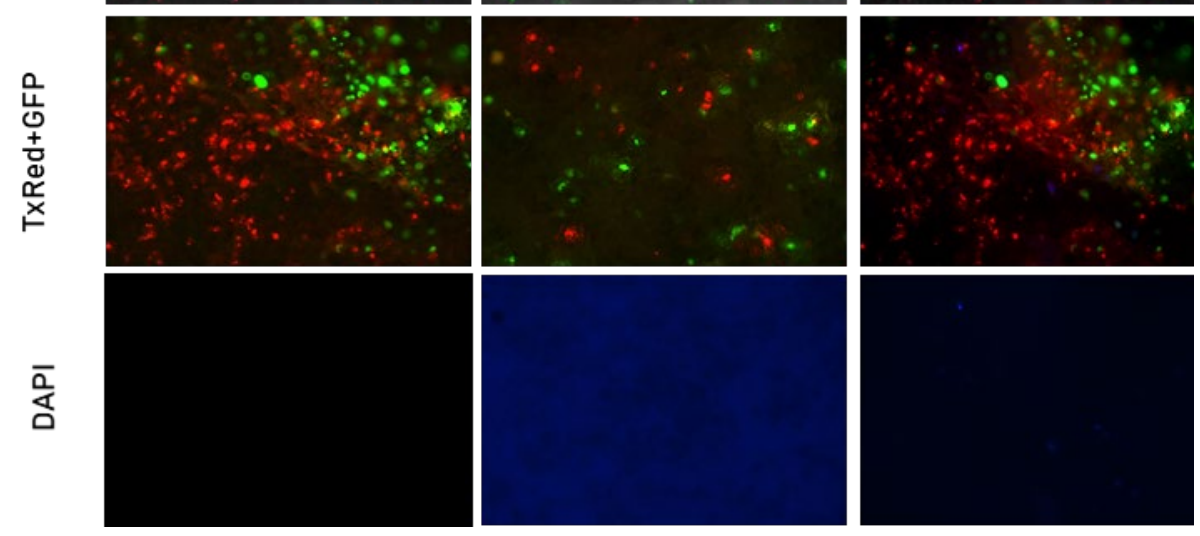

Figure S22. 184B5 cells in cell tracker green and MDA-MB-231 cells in cell tracker red seeded in coculture on mesh scaffolds and imaged in phase, TxRed and DAPI (A) 24 hours with and without $20 \mu \mathrm{M}$ ManCou-H media and scaffolds containing ManCou-H (B) 48 
hours with and without $20 \mu \mathrm{M}$ ManCou-H media and scaffolds containing ManCou-H. Images captured at $10 \mathrm{x}$ magnification. DAPI images in conditions without $\mathrm{ManCou}-\mathrm{H}$ were not taken.
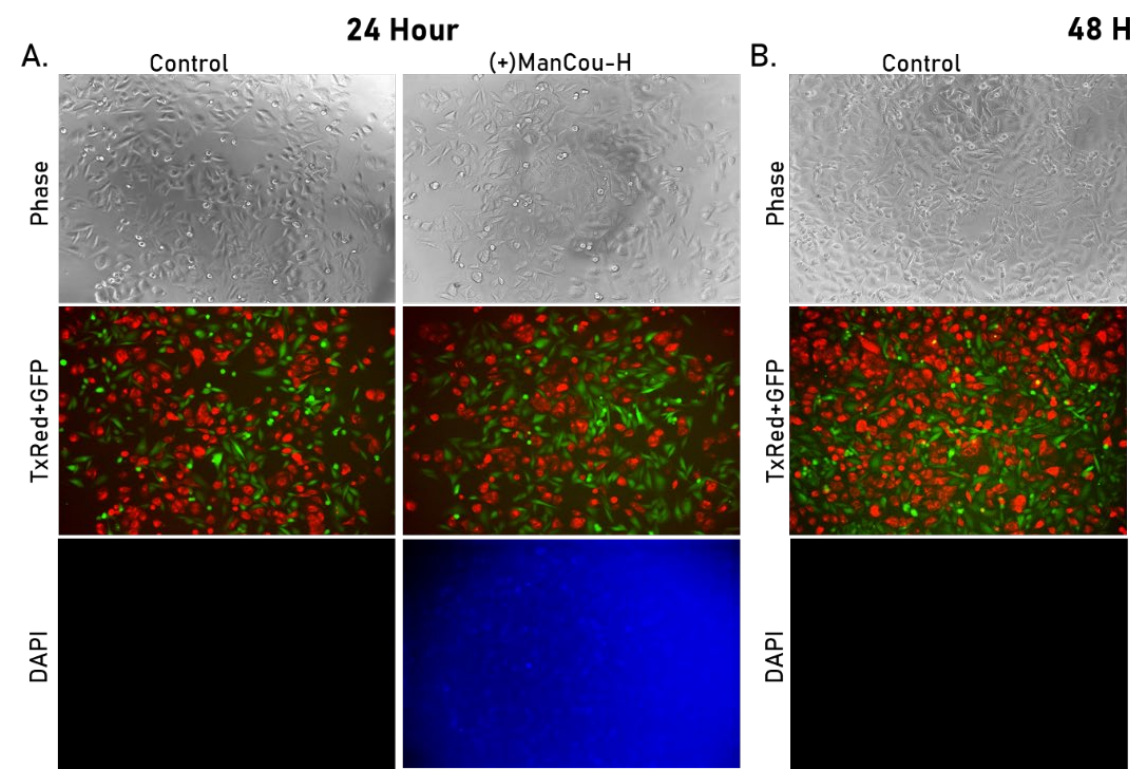

$(+)$ ManCou-H

Figure S23. MDA-MB-231 cells stained with cell tracker green and MCF7 cells stained with cell tracker red seeded in coculture on 2D culture plate imaged in phase, TxRed and DAPI (A) 24-hour time interval on control (no ManCou-H) and plates supplemented with $20 \mu \mathrm{M}$ ManCou-H (B) 48-hour time interval on control (no ManCou-H) and plates supplemented with $20 \mu \mathrm{M}$ ManCou-H. Images captured at 10x magnification. Images in DAPI on control were not taken. 

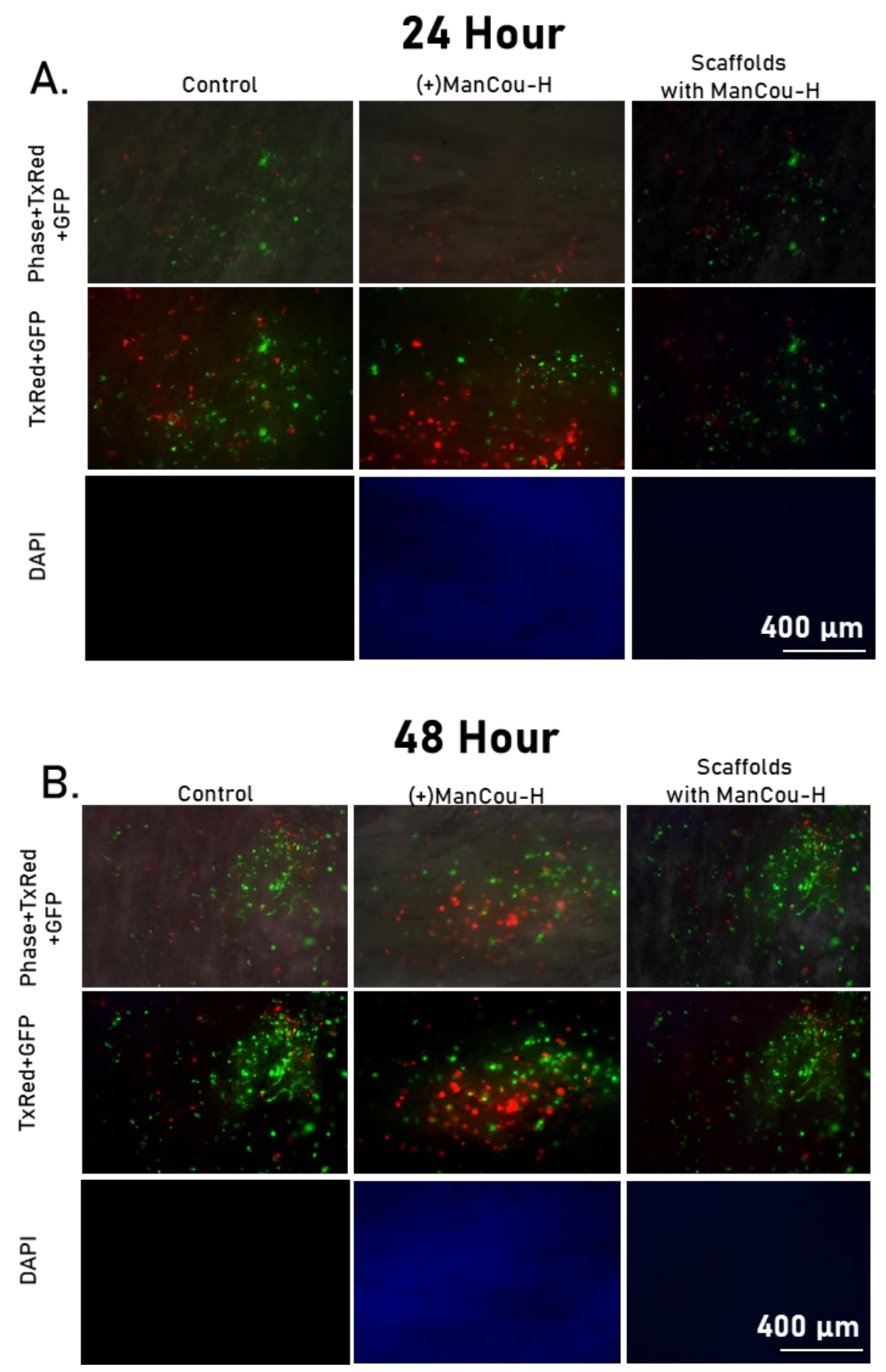

Figure S24. MDA-MB-231 cells in cell tracker green and MCF7 cells in cell tracker red seeded in coculture on aligned scaffolds and imaged in phase, TxRed and DAPI (A) 24 hours with and without $20 \mu \mathrm{M}$ ManCou-H media and scaffolds containing ManCou-H (B) 48 hours with and without $20 \mu \mathrm{M}$ ManCou-H media and scaffolds containing ManCou- 
H. Images captured at 10x magnification. DAPI images in conditions without ManCou-H were not taken.

\section{Hour}
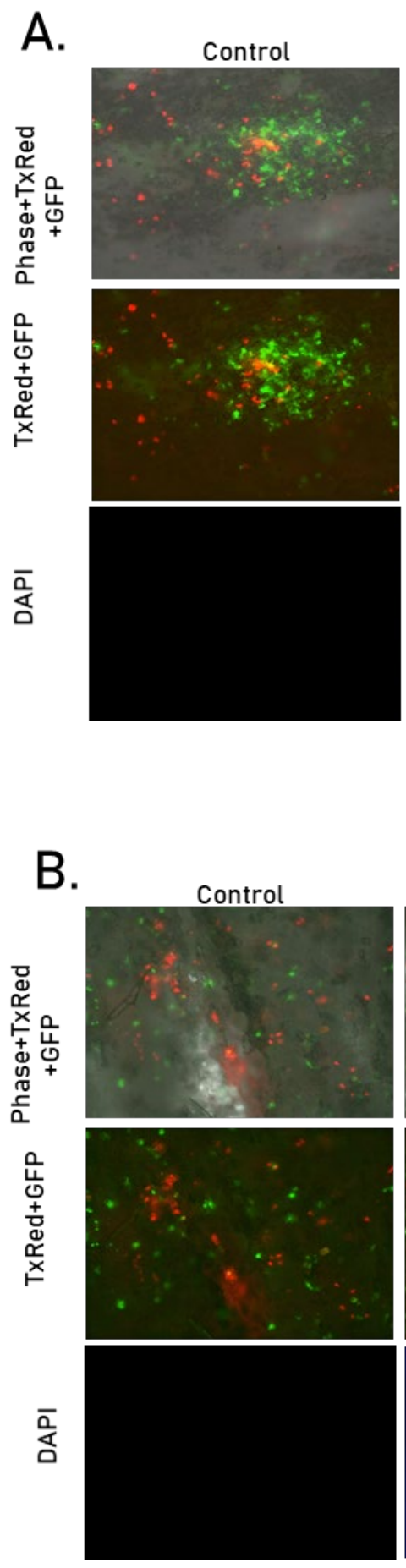
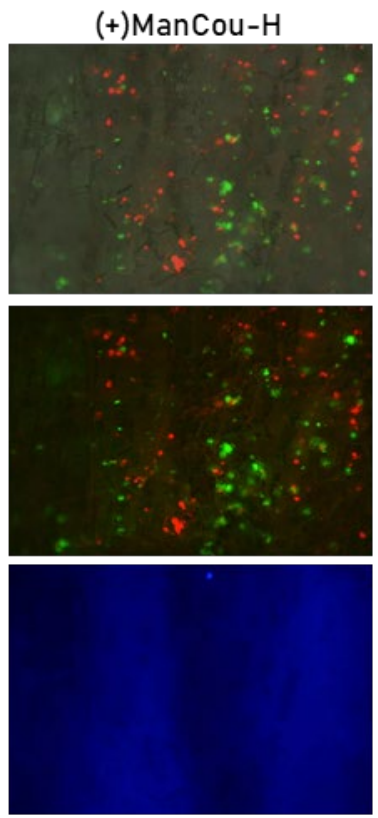

\section{Hour}

Scaffolds with ManCou-H
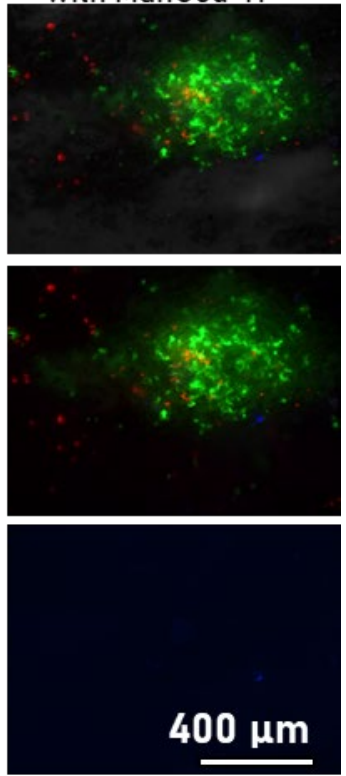

(+)ManCou-H
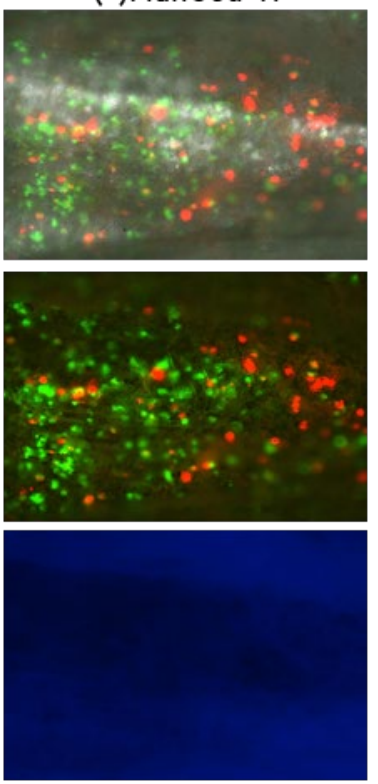

Scaffolds with ManCou-H
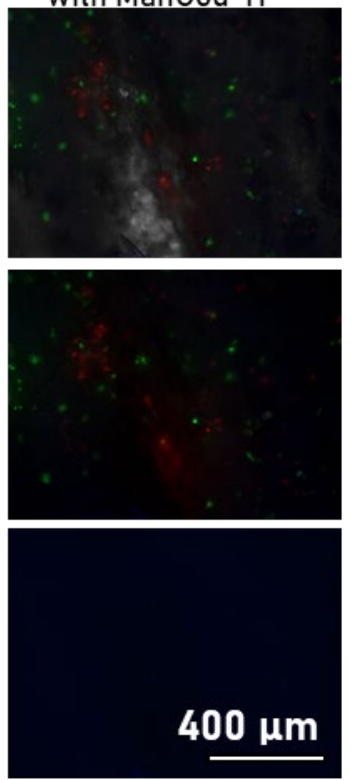
Figure S25. MDA-MB-231 cells in cell tracker green and MCF7 cells in cell tracker red seeded in coculture on honeycomb scaffolds and imaged in Phase, TxRed and DAPI (A) 24 hours with and without $20 \mu \mathrm{M}$ ManCou- $\mathrm{H}$ media and scaffolds containing ManCou-H (B) 48 hours with and without $20 \mu \mathrm{M}$ ManCou-H media and scaffolds containing ManCou$\mathrm{H}$. Images captured at 10x magnification. DAPI images in conditions without $\mathrm{ManCou}-\mathrm{H}$ were not taken.

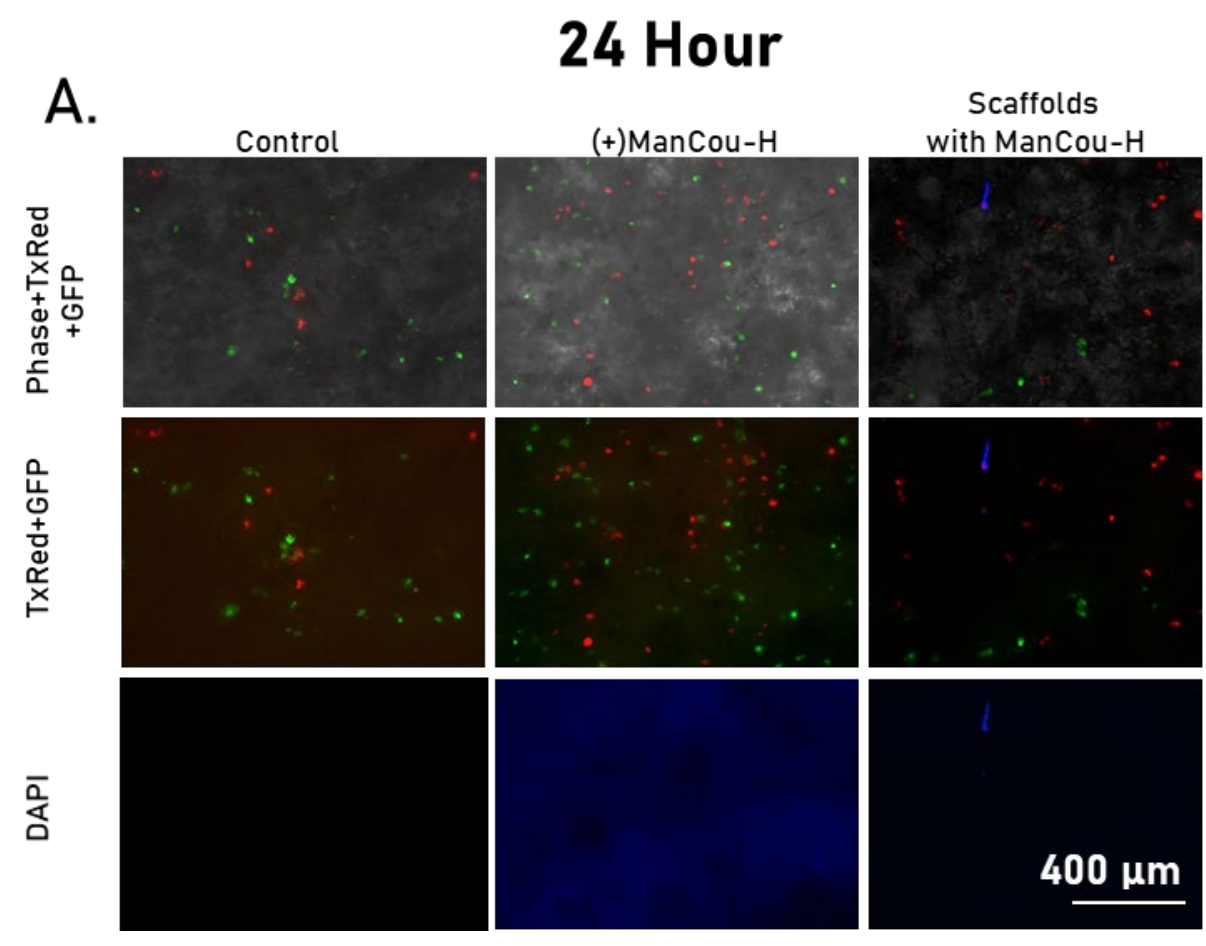




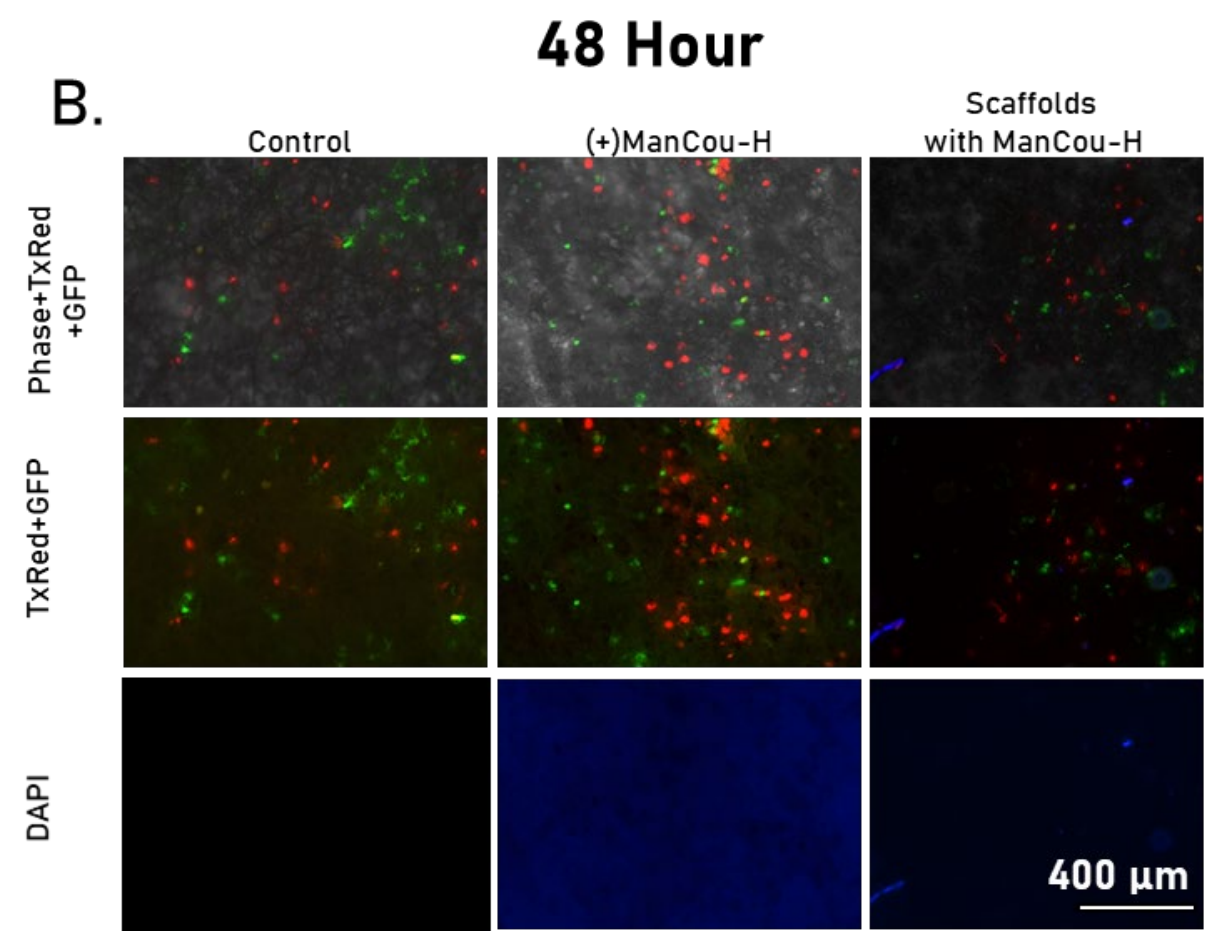

Figure S26. MDA-MB-231 cells in cell tracker green and MCF7 cells in cell tracker red seeded in coculture on mesh scaffolds and imaged in phase, TxRed and DAPI (A) 24 hours with and without $20 \mu \mathrm{M}$ ManCou-H media and scaffolds containing ManCou-H (B) 48 hours with and without $20 \mu \mathrm{M}$ ManCou-H media and scaffolds containing ManCou-H. Images captured at 10x magnification. DAPI images in conditions without ManCou-H were not taken.
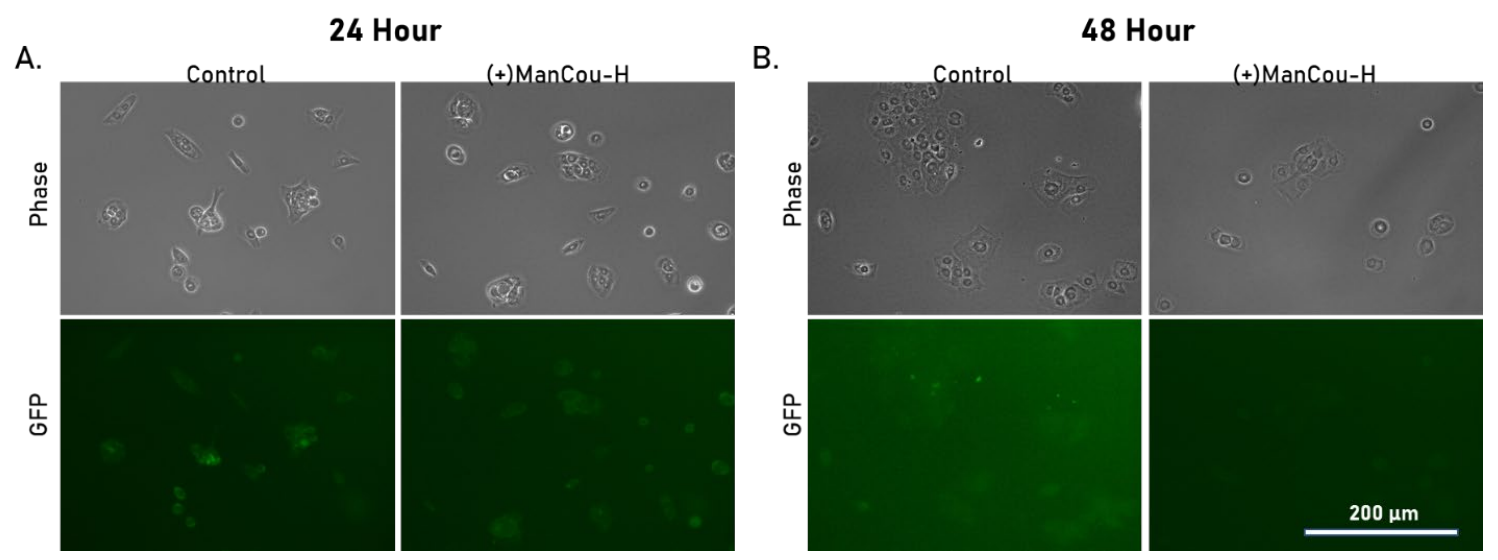

Figure S27 184B5 cells seeded on 2D culture plates and immunostained for GLUT5 imaged in phase and GFP (A) 24 hours with and without $20 \mu \mathrm{M}$ ManCou-H media (B) 48 hours with and without $20 \mu \mathrm{M}$ ManCou-H media. Images captured at 20x magnification. 

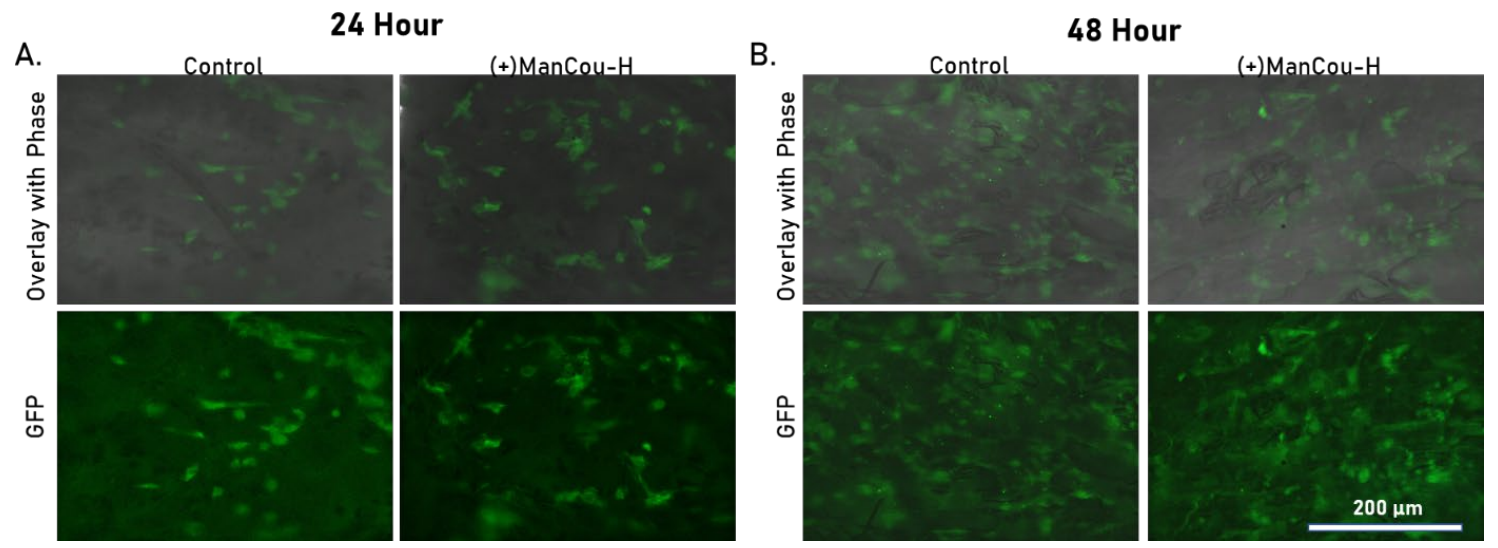

Figure S28 184B5 cells seeded on aligned scaffolds and immunostained for GLUT5. Imaged in phase and GFP (A) 24 hours with and without $20 \mu \mathrm{M}$ ManCou-H media (B) 48 hours with and without $20 \mu \mathrm{M}$ ManCou-H media. Images captured at 20x magnification.
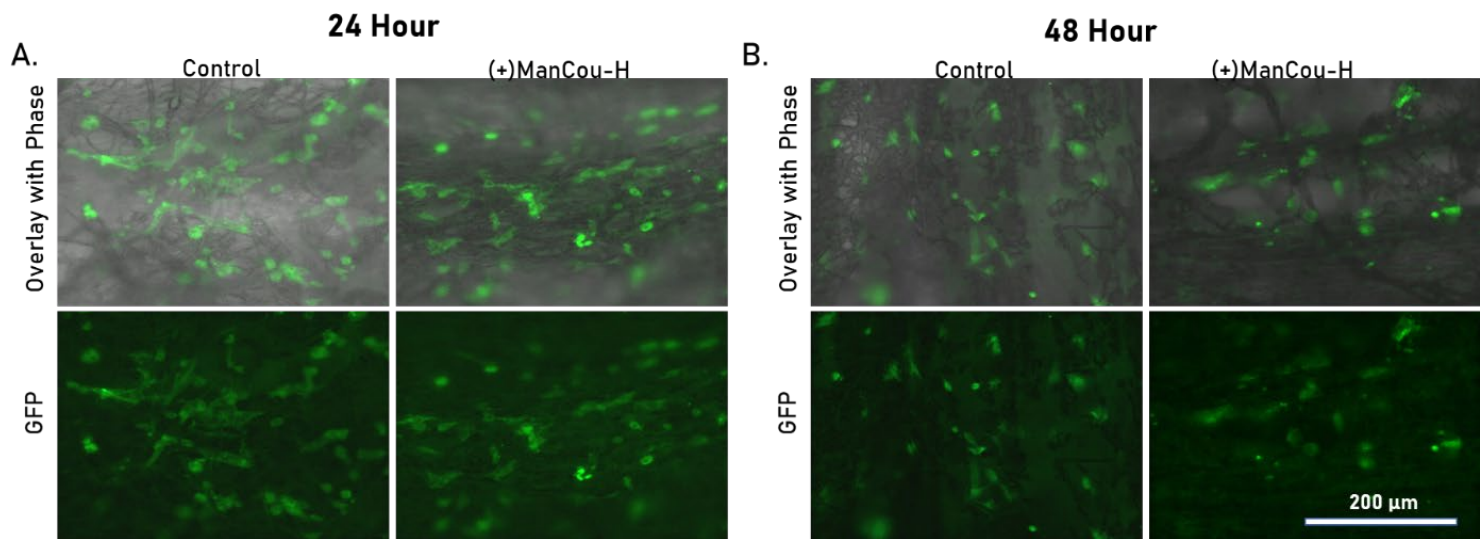

Figure S29 184B5 cells seeded on honeycomb scaffolds and immunostained for GLUT5. Imaged in phase and GFP (A) 24 hours with and without $20 \mu \mathrm{M}$ ManCou-H media (B) 48 hours with and without $20 \mu \mathrm{M}$ ManCou-H media. Images captured at 20x magnification. 

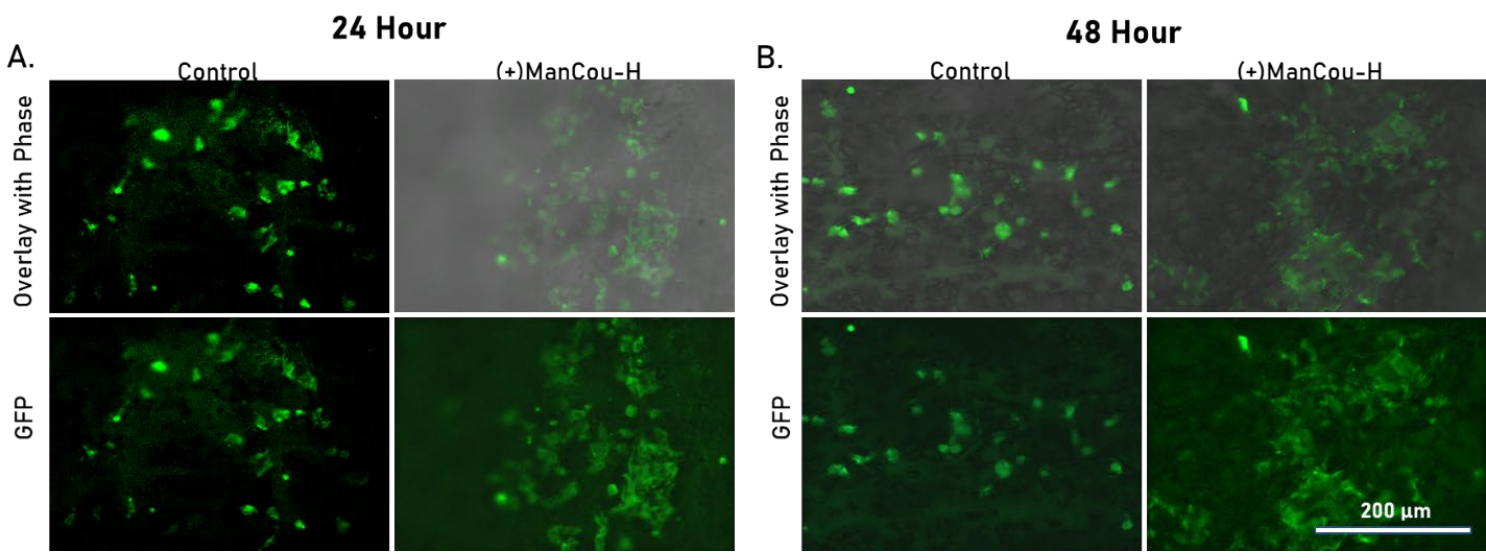

Figure S30 184B5 cells seeded on mesh scaffolds and immunostained for GLUT5. Imaged in phase and GFP (A) 24 hours with and without $20 \mu \mathrm{M}$ ManCou-H media (B) 48 hours with and without $20 \mu \mathrm{M}$ ManCou-H media. Images captured at 20x magnification.
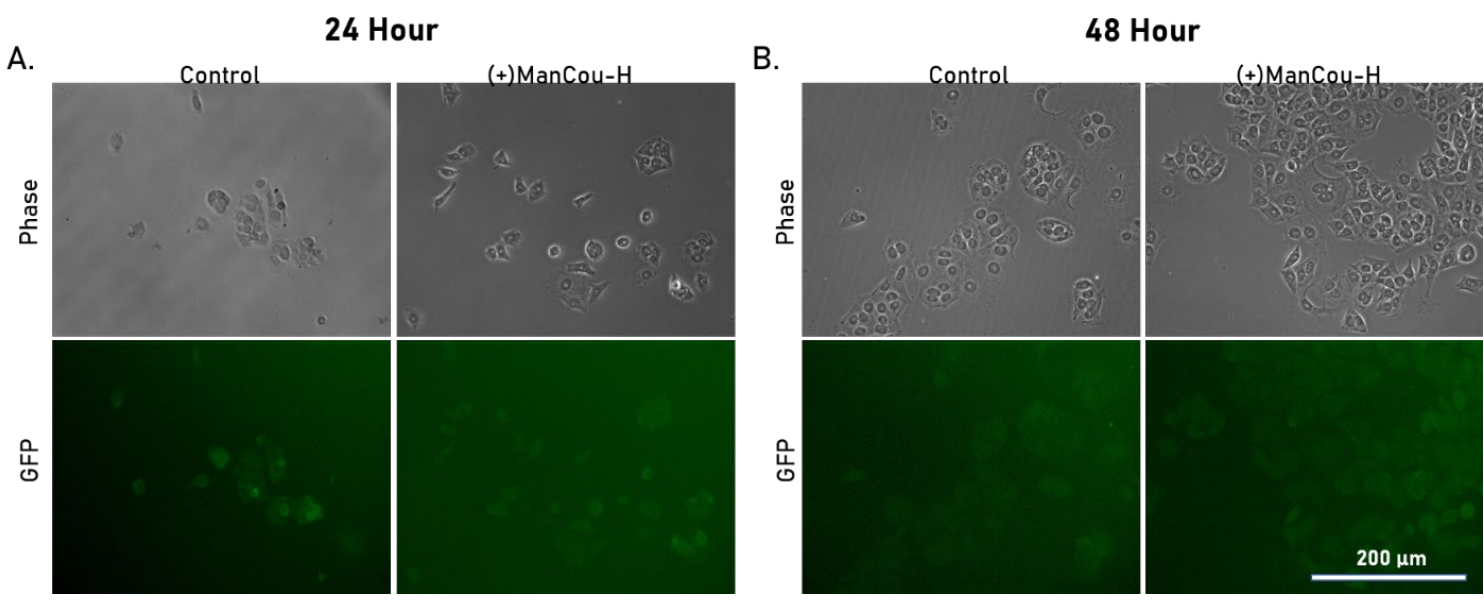

Figure S31 MCF7 cells seeded on 2D culture plates and immunostained for GLUT5 imaged in phase and GFP (A) 24 hours with and without $20 \mu \mathrm{M}$ ManCou-H media (B) 48 hours with and without $20 \mu \mathrm{M}$ ManCou-H media. Images captured at 20x magnification. 

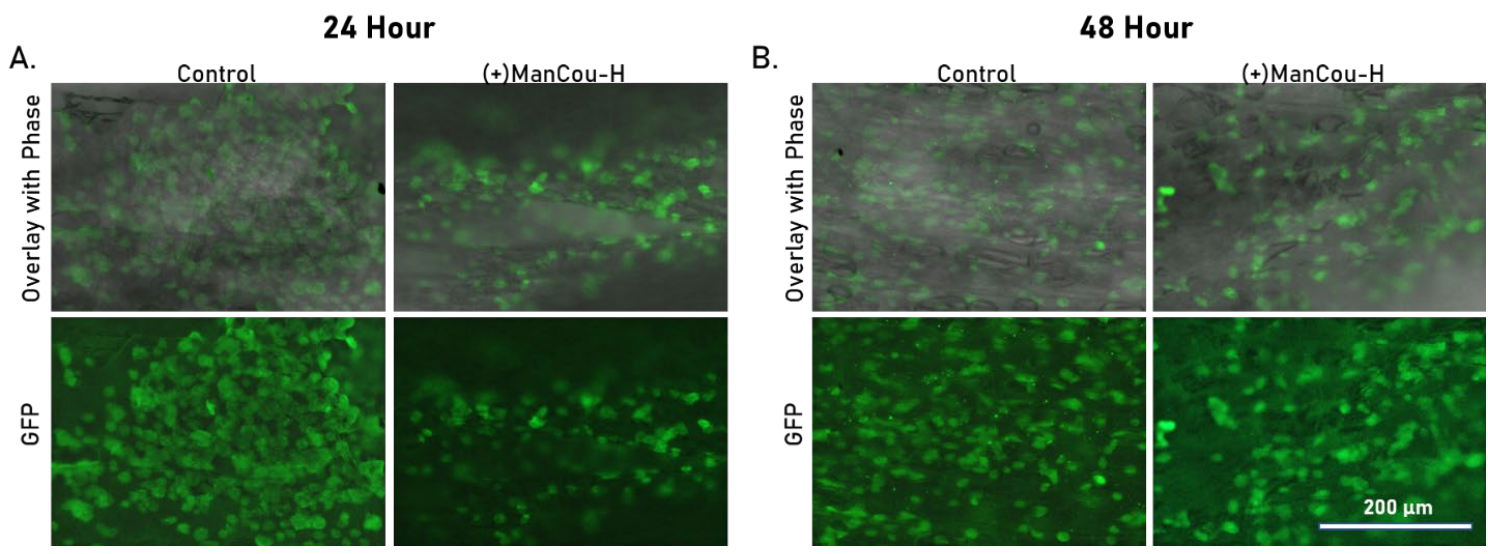

Figure S32 MCF7 cells seeded on aligned scaffolds and immunostained for GLUT5. Imaged in phase and GFP (A) 24 hours with and without $20 \mu \mathrm{M}$ ManCou-H media (B) 48 hours with and without $20 \mu \mathrm{M}$ ManCou-H media. Images captured at 20x magnification.
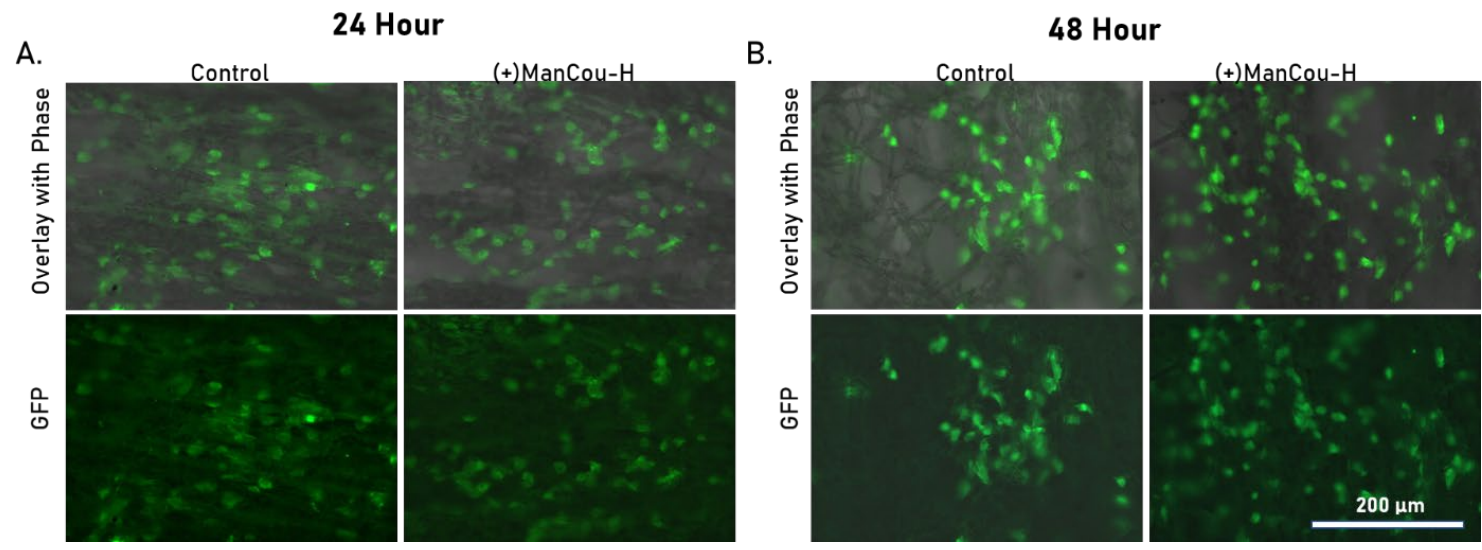

Figure S33 MCF7 cells seeded on honeycomb scaffolds and immunostained for GLUT5. Imaged in phase and GFP (A) 24 hours with and without $20 \mu \mathrm{M}$ ManCou-H media (B) 48 hours with and without $20 \mu \mathrm{M}$ ManCou-H media. Images captured at 20x magnification.
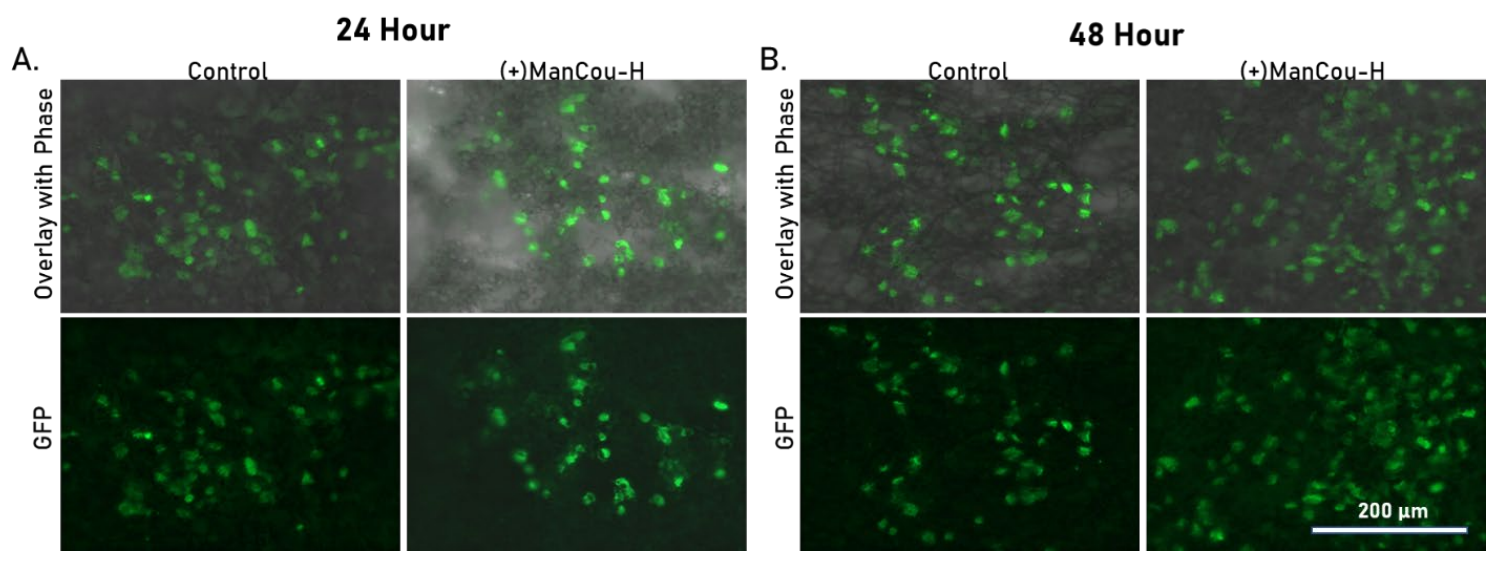
Figure S34 MCF7 cells seeded on mesh scaffolds and immunostained for GLUT5. Imaged in phase and GFP (A) 24 hours with and without $20 \mu \mathrm{M}$ ManCou-H media (B) 48 hours with and without $20 \mu \mathrm{M}$ ManCou-H media. Images captured at 20x magnification.
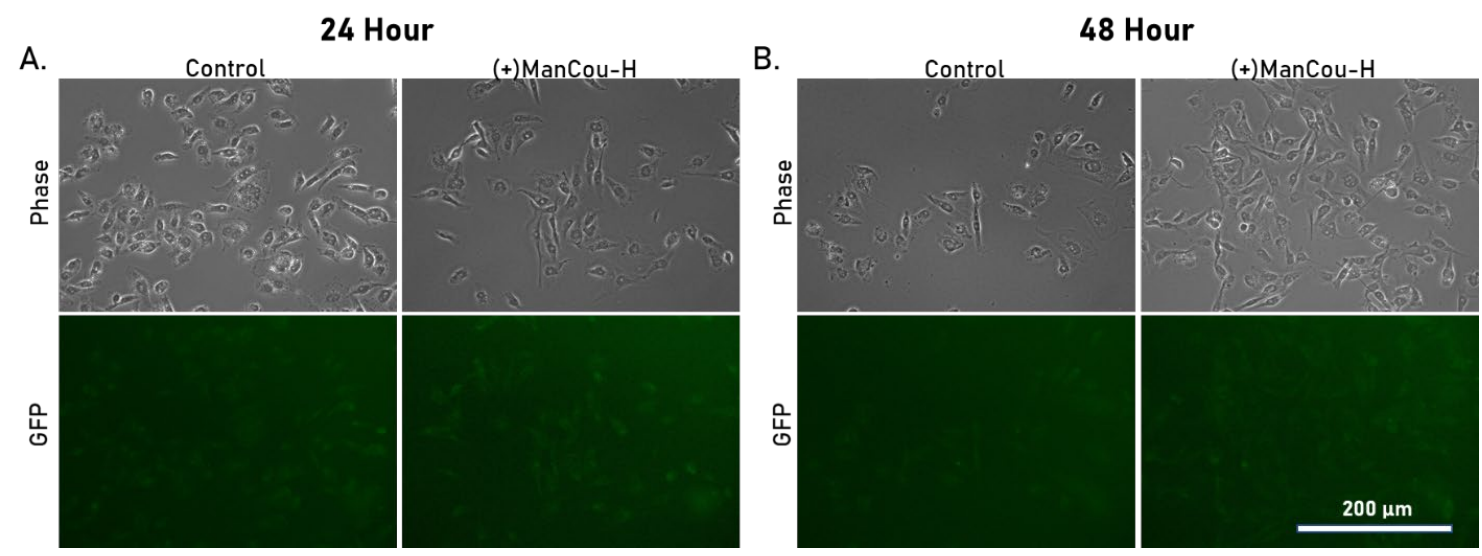

Figure S35 MDA-MB-231 cells seeded on culture plates and immunostained for GLUT5 imaged in phase and GFP (A) 24 hours with and without $20 \mu \mathrm{M}$ ManCou-H media (B) 48 hours with and without $20 \mu \mathrm{M}$ ManCou-H media. Images captured at 20x magnification.
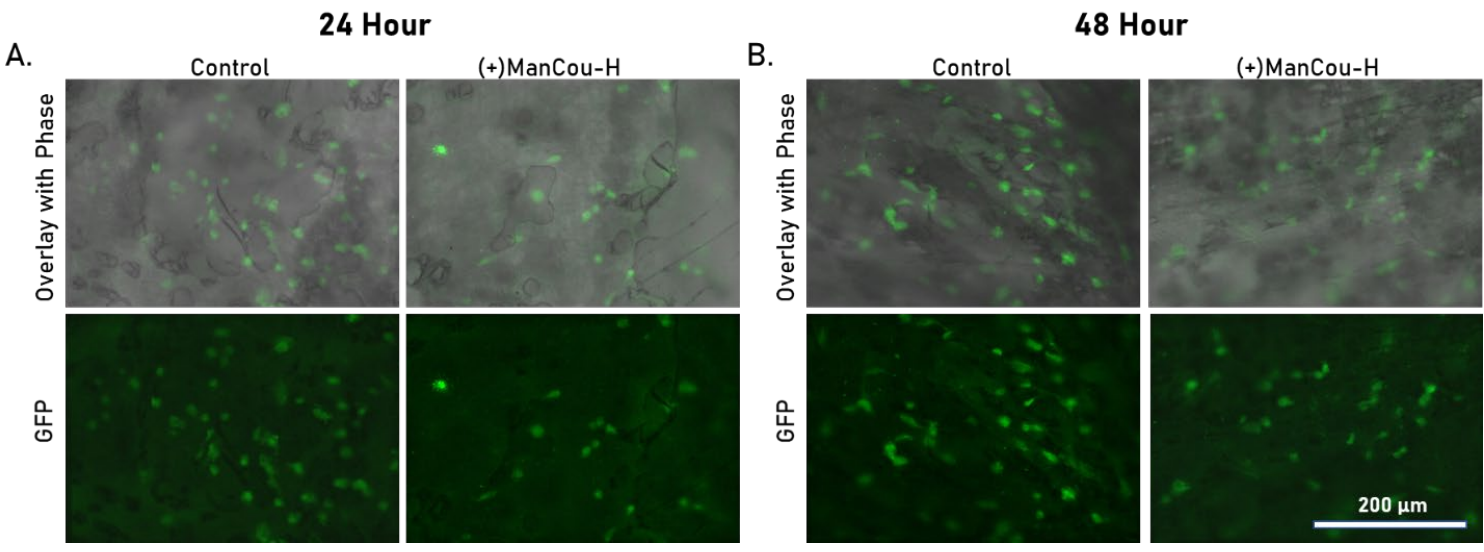

Figure S36. MDA-MB-231 cells seeded on aligned fiber scaffolds and immuno-stained for GLUT5. Imaged in phase and GFP (A) 24 hours with and without $20 \mu \mathrm{M}$ ManCou-H media (B) 48 hours with and without $20 \mu \mathrm{M}$ ManCou-H media. Images captured at $20 \mathrm{x}$ magnification. 

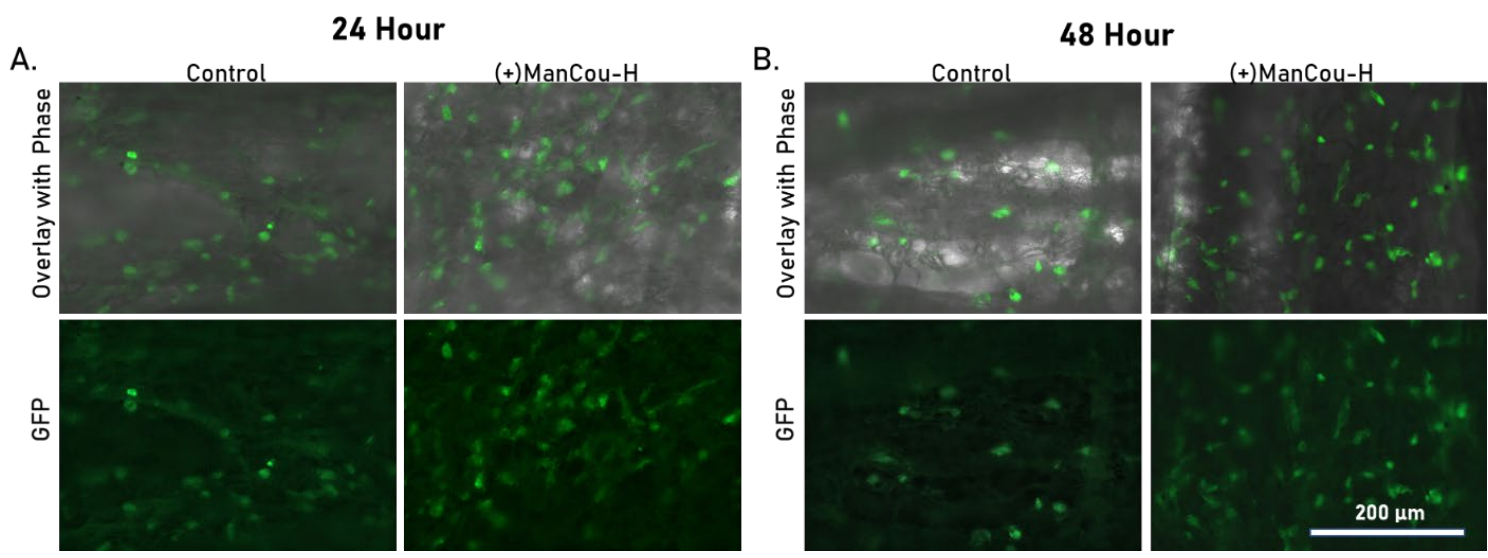

Figure S37. MDA-MB-231 cells seeded on honeycomb fiber scaffolds and immunostained for GLUT5. Imaged in phase and GFP (A) 24 hours with and without $20 \mu \mathrm{M}$ ManCou-H media (B) 48 hours with and without $20 \mu \mathrm{M}$ ManCou-H media. Images captured at 20x magnification.
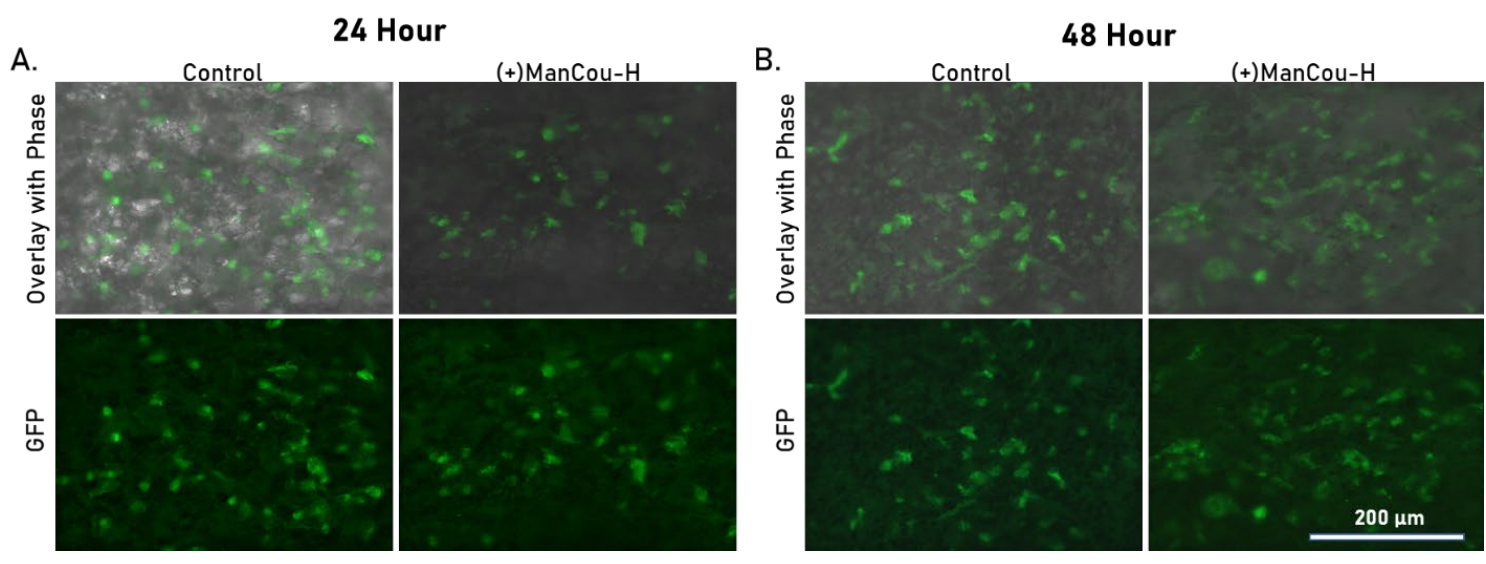

Figure S38. MDA-MB-231 cells seeded on mesh fiber scaffolds and immuno-stained for GLUT5. Imaged in phase and GFP (A) 24 hours with and without $20 \mu \mathrm{M}$ ManCou-H media (B) 48 hours with and without $20 \mu \mathrm{M}$ ManCou-H media. Images captured at $20 \mathrm{x}$ magnification. 

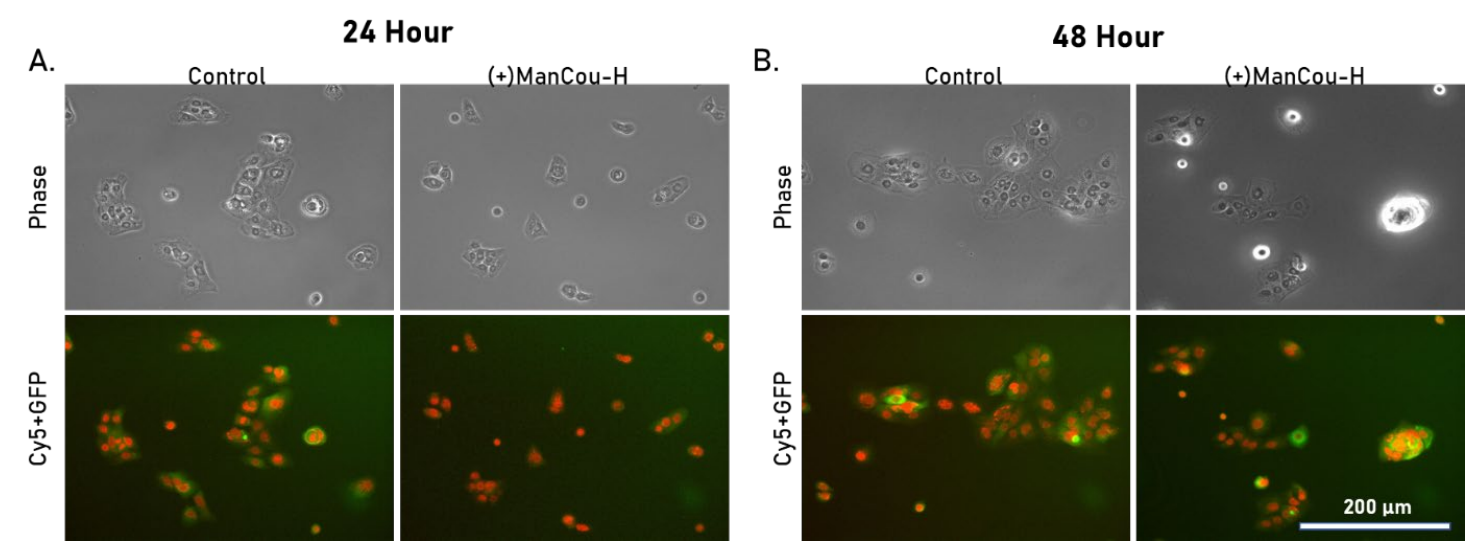

Figure S39. 184B5 cells seeded on culture plates and immuno-stained for cytokeratin-18, with the nucleus stained red. Imaged in phase, GFP, and Cy5 (A) 24 hours with and without $20 \mu \mathrm{M}$ ManCou-H media (B) 48 hours with and without $20 \mu \mathrm{M}$ ManCou-H media. Images captured at 20x magnification.
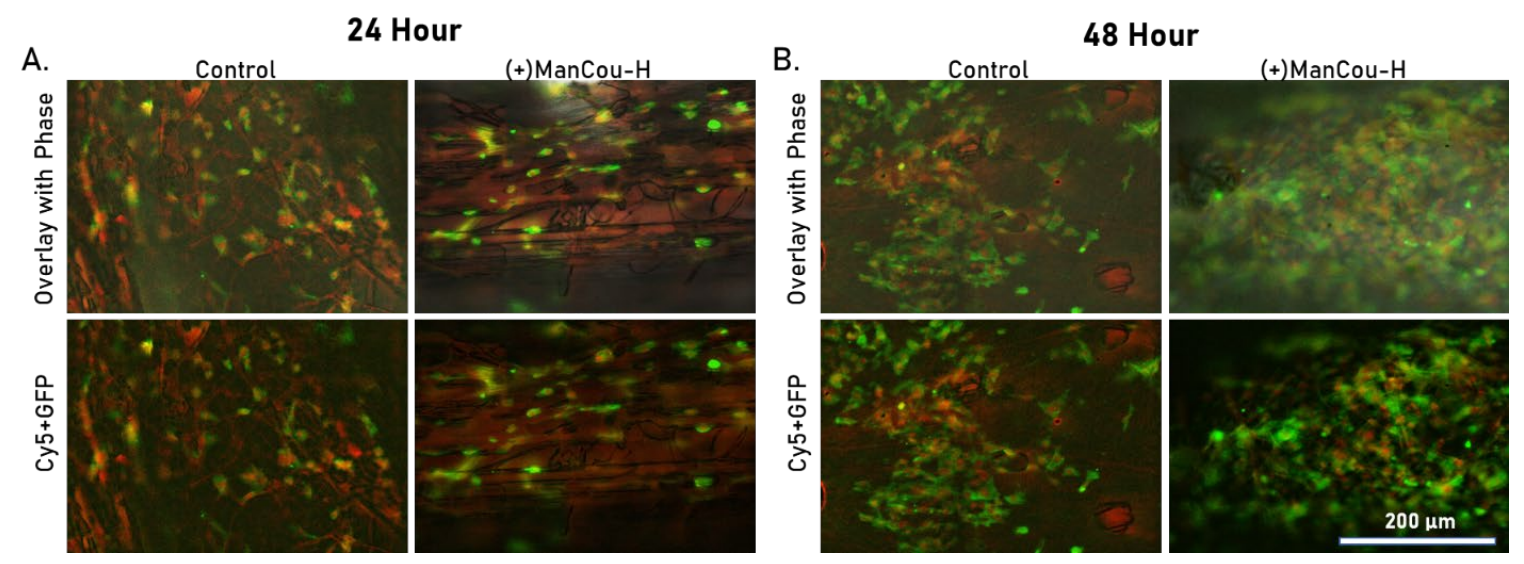

Figure S40. 184B5 cells seeded on aligned fiber scaffolds and immuno-stained for cytokeratin-18, with the nucleus stained red. Imaged in phase, GFP and Cy5 (A) 24 hours with and without $20 \mu \mathrm{M}$ ManCou-H media (B) 48 hours with and without $20 \mu \mathrm{M}$ ManCou$\mathrm{H}$ media. Images captured at 20x magnification. 

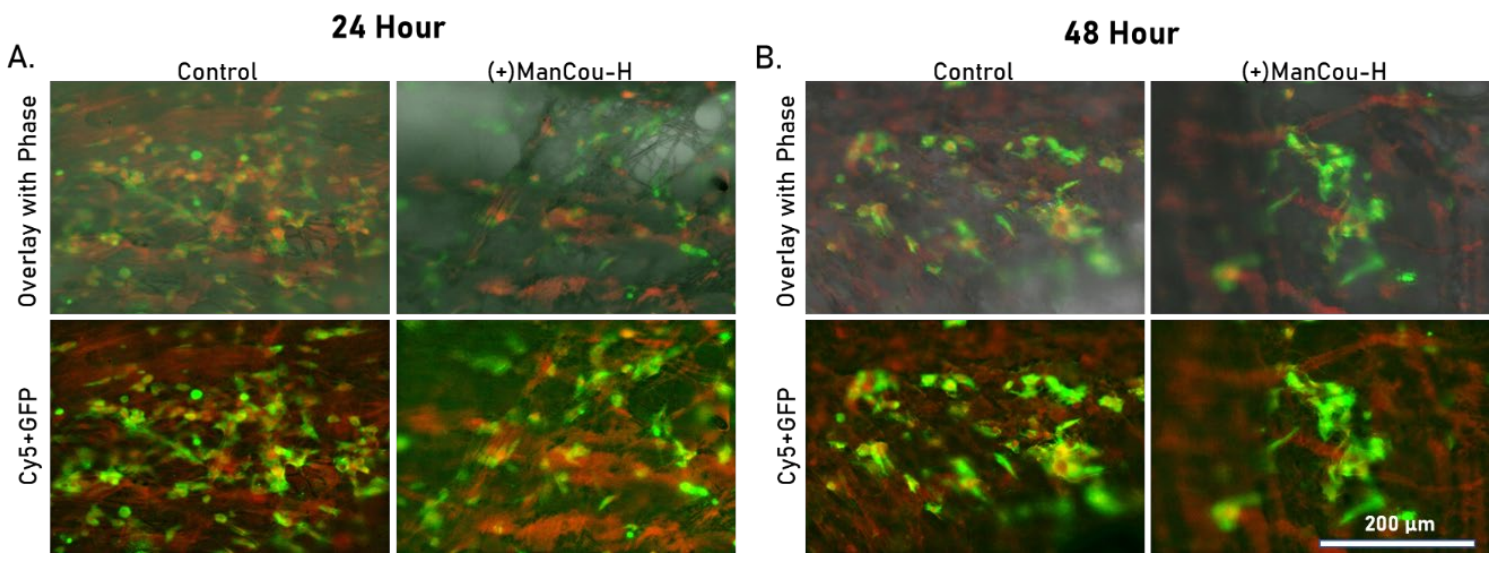

Figure S41. 184B5 cells seeded on honeycomb fiber scaffolds and immuno-stained for cytokeratin-18, with the nucleus stained red. Imaged in phase, GFP and Cy5 (A) 24 hours with and without $20 \mu \mathrm{M}$ ManCou-H media (B) 48 hours with and without $20 \mu \mathrm{M}$ ManCou$\mathrm{H}$ media. Images captured at 20x magnification.
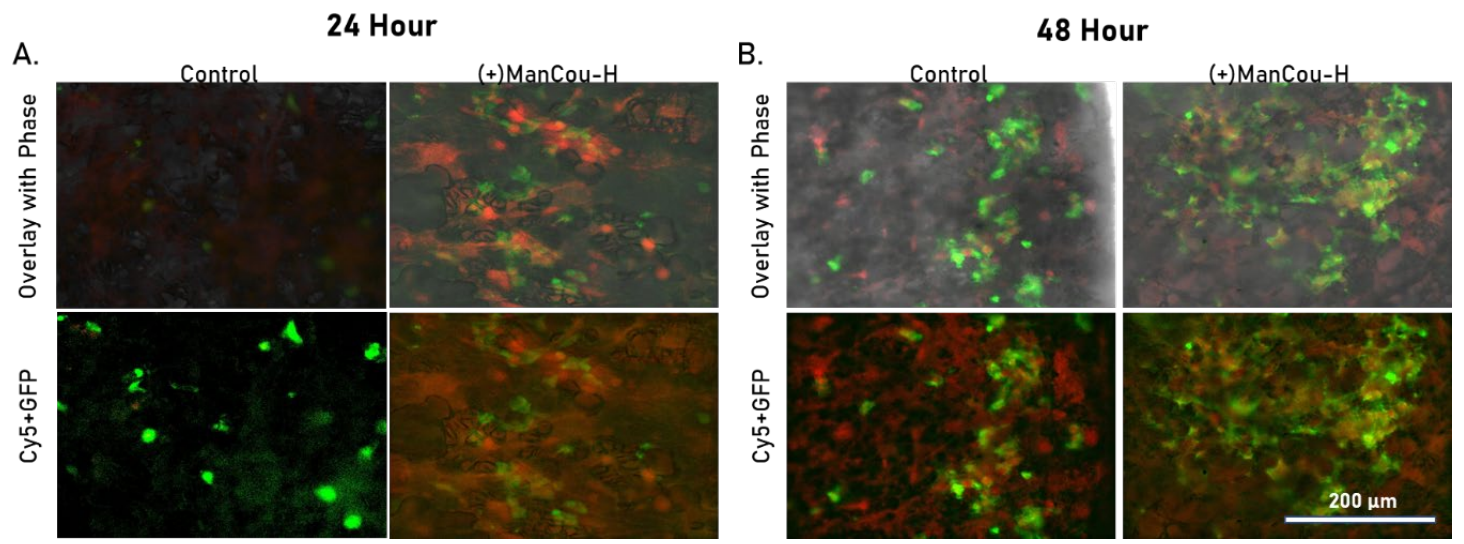

Figure S42. 184B5 cells seeded on mesh fiber scaffolds and immuno-stained for cytokeratin-18, with the nucleus stained red. Imaged in phase, GFP and Cy5 (A) 24 hours with and without $20 \mu \mathrm{M}$ ManCou-H media (B) 48 hours with and without $20 \mu \mathrm{M}$ ManCou$\mathrm{H}$ media. Images captured at 20x magnification. 

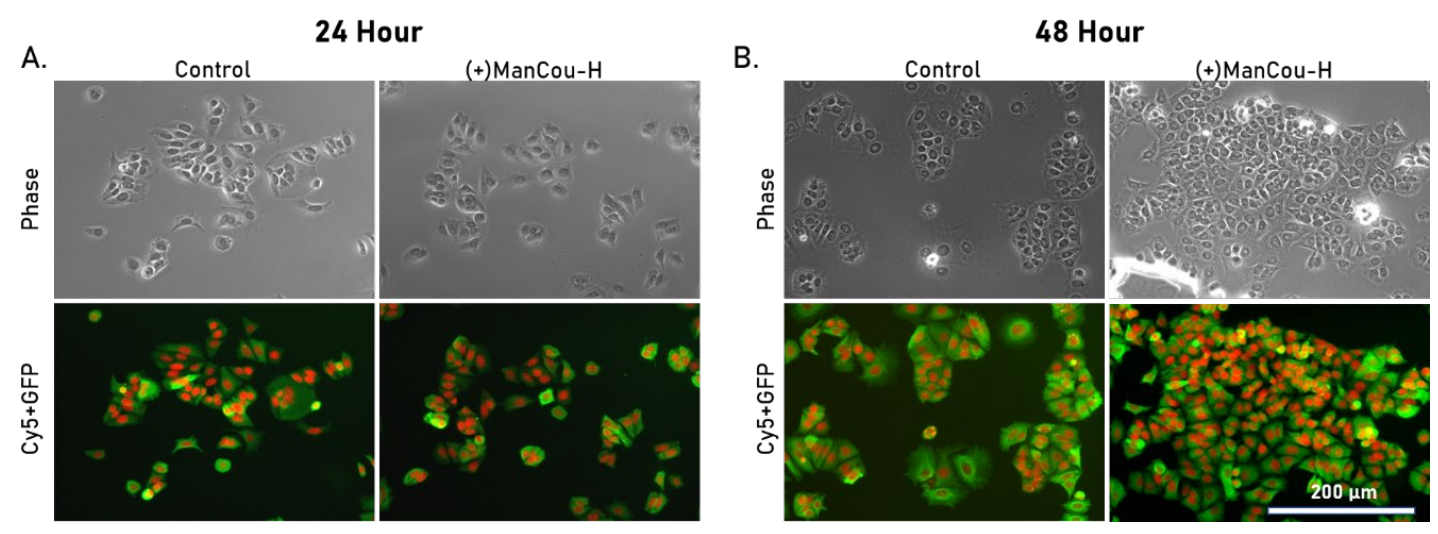

Figure S43. MCF7 cells seeded on culture plates and immuno-stained for cytokeratin-18, with the nucleus stained red. Imaged in phase, GFP and Cy5 (A) 24 hours with and without $20 \mu \mathrm{M}$ ManCou-H media (B) 48 hours with and without $20 \mu \mathrm{M}$ ManCou-H media. Images captured at 20x magnification.
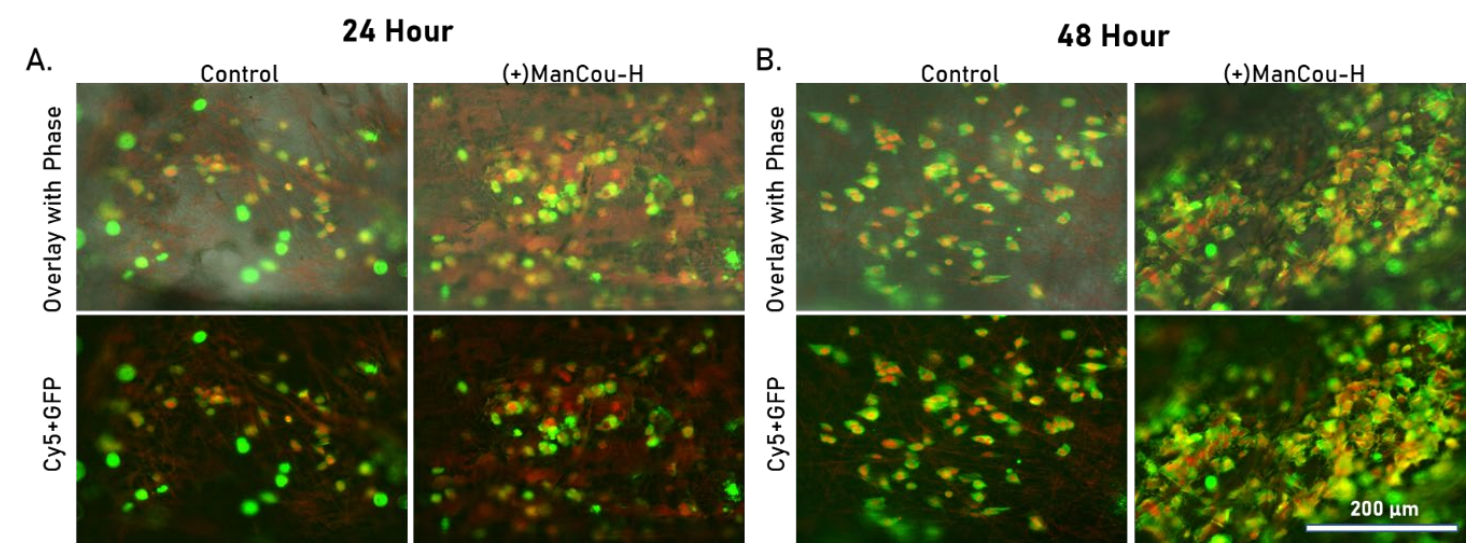

Figure S44. MCF7 cells seeded on aligned fiber scaffolds and immuno-stained for cytokeratin-18, with the nucleus stained red. Imaged in phase, GFP and Cy5 (A) 24 hours with and without $20 \mu \mathrm{M}$ ManCou-H media (B) 48 hours with and without $20 \mu \mathrm{M}$ ManCou$\mathrm{H}$ media. Images captured at 20x magnification.
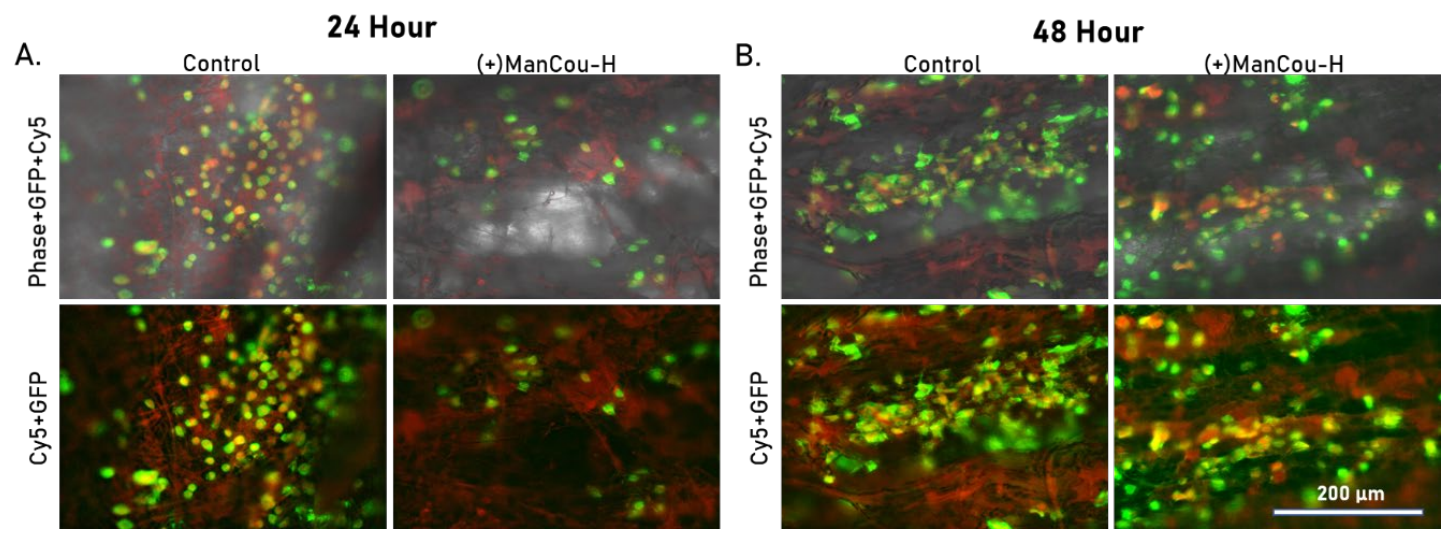
Figure S45. MCF7 cells seeded on honeycomb fiber scaffolds and immuno-stained for cytokeratin-18, with the nucleus stained red. Imaged in phase, GFP and Cy5 (A) 24 hours with and without $20 \mu \mathrm{M}$ ManCou-H media (B) 48 hours with and without $20 \mu \mathrm{M}$ ManCou$\mathrm{H}$ media. Images captured at 20x magnification.
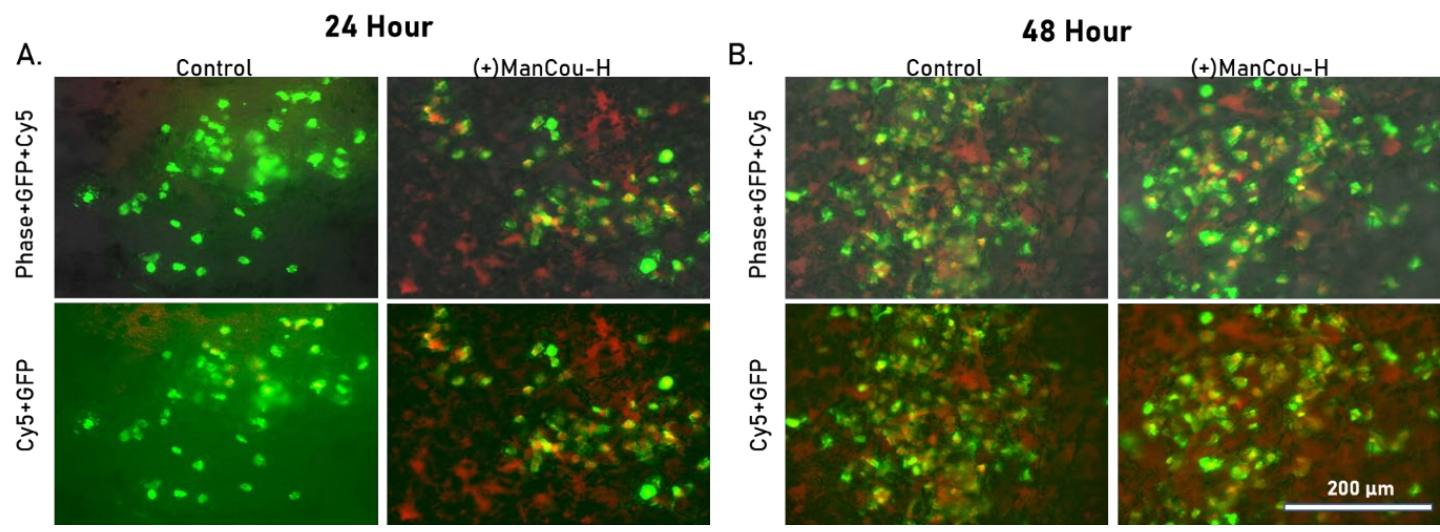

Figure S46. MCF7 cells seeded on mesh fiber scaffolds and immuno-stained for cytokeratin-18, with the nucleus stained red. Imaged in phase, GFP and Cy5 (A) 24 hours with and without $20 \mu \mathrm{M}$ ManCou-H media (B) 48 hours with and without $20 \mu \mathrm{M}$ ManCou$\mathrm{H}$ media. Images captured at 20x magnification.
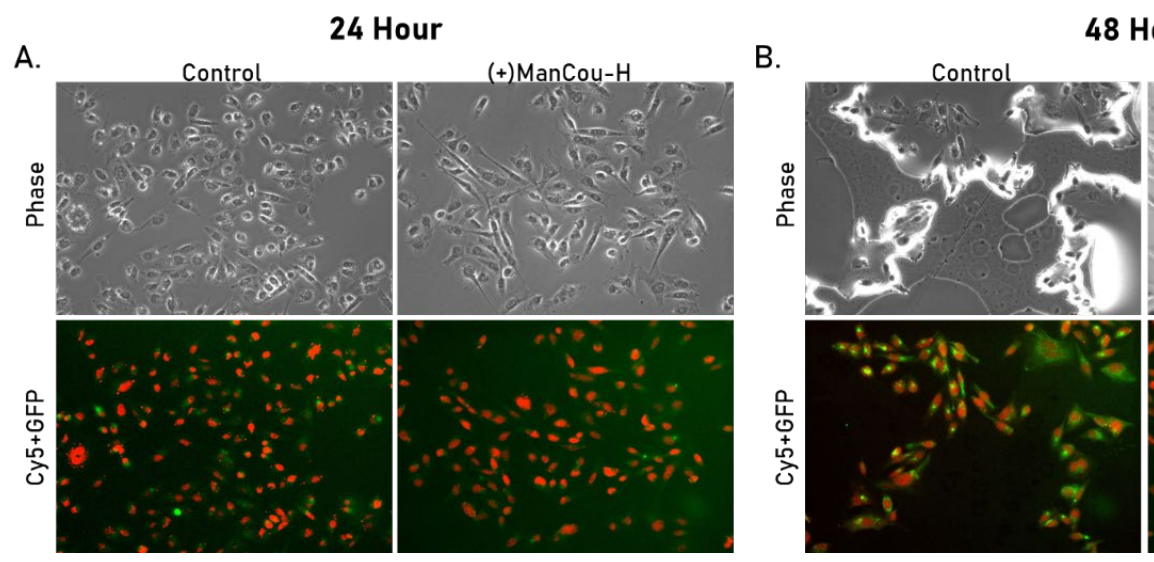

48 Hour

Figure S47. MDA-MB-231 cells seeded on culture plates and immuno-stained for cytokeratin-18, with the nucleus stained red. Imaged in phase, GFP and Cy5 (A) 24 hours with and without $20 \mu \mathrm{M}$ ManCou-H media (B) 48 hours with and without $20 \mu \mathrm{M}$ ManCou$\mathrm{H}$ media. Images captured at 20x magnification. 

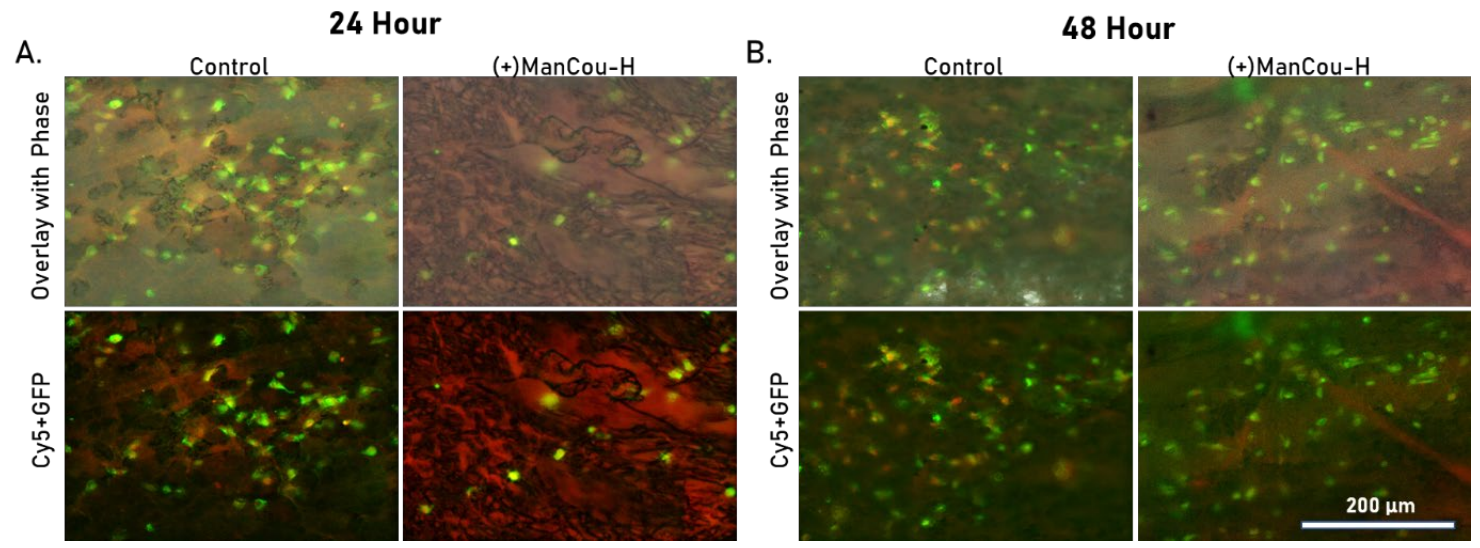

Figure S48. MDA-MB-231 cells seeded on aligned fiber scaffolds and immuno-stained for cytokeratin-18, with the nucleus stained red. Imaged in phase, GFP and Cy5 (A) 24 hours with and without $20 \mu \mathrm{M}$ ManCou-H media (B) 48 hours with and without $20 \mu \mathrm{M}$ ManCou$\mathrm{H}$ media. Images captured at 20x magnification.
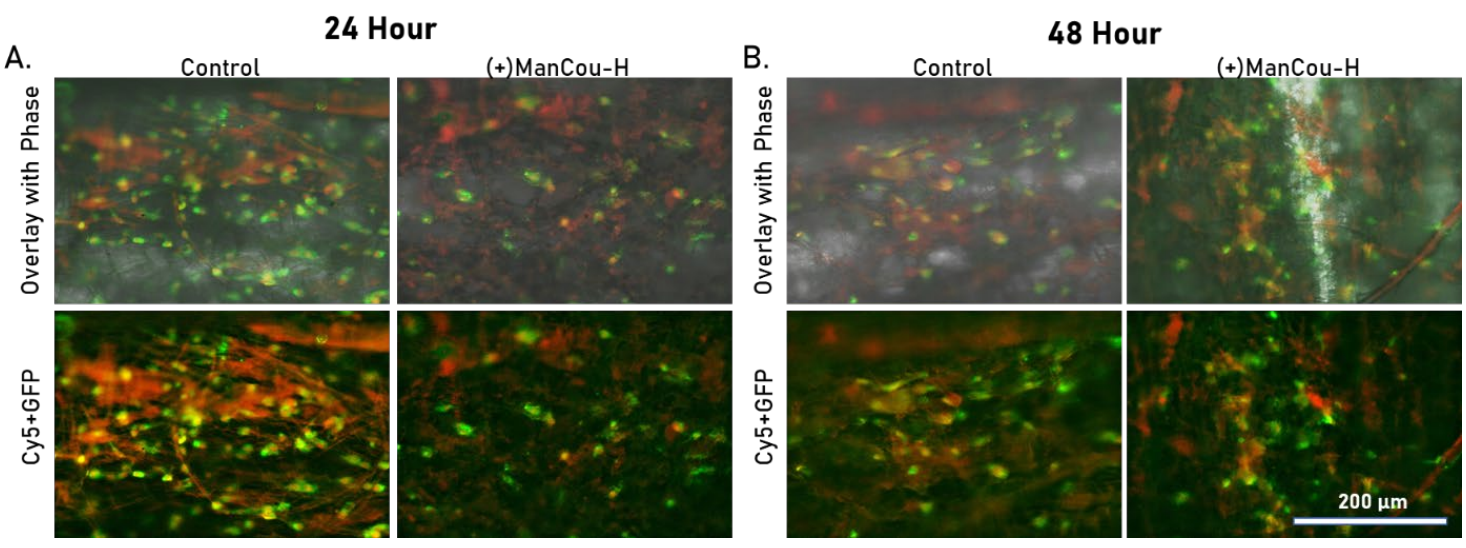

Figure S49. MDA-MB-231 cells seeded on honeycomb fiber scaffolds and immunostained for cytokeratin-18, with the nucleus stained red. Imaged in phase and Cy5 (A) 24 hours with and without $20 \mu \mathrm{M}$ ManCou-H media (B) 48 hours with and without $20 \mu \mathrm{M}$ ManCou-H media. Images captured at 20x magnification. 

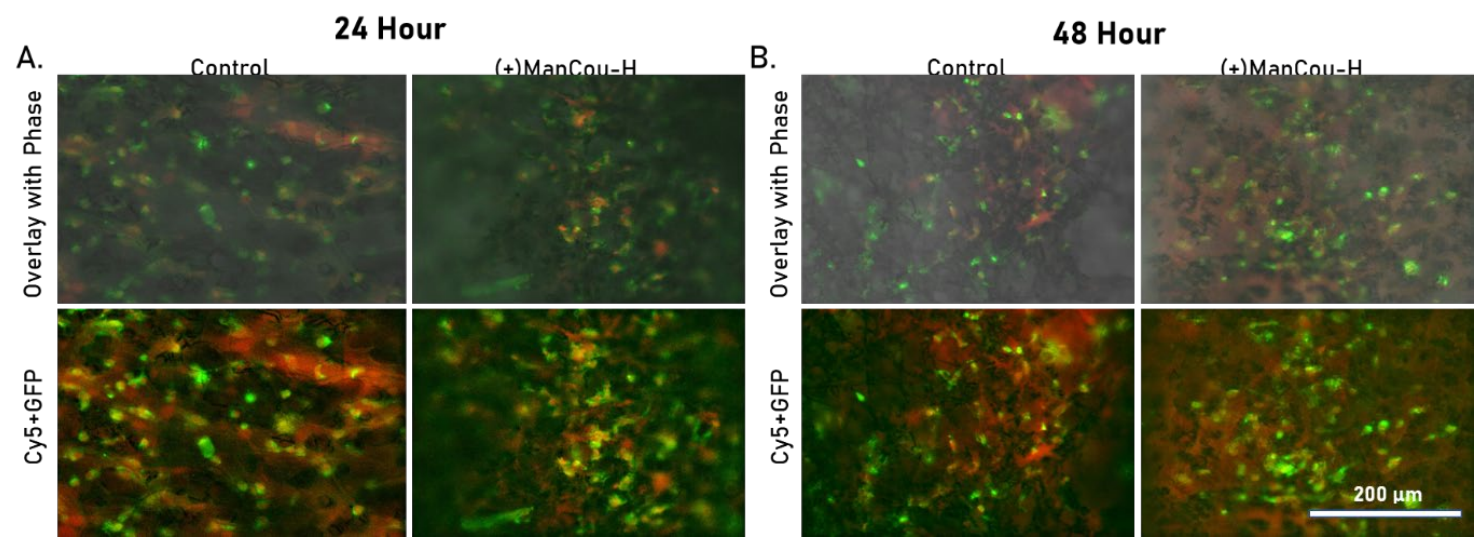

Figure S50. MDA-MB-231 cells seeded on mesh fiber scaffolds and immuno-stained for cytokeratin-18, with the nucleus stained red. Imaged in phase, GFP and Cy5 (A) 24 hours with and without $20 \mu \mathrm{M}$ ManCou-H media (B) 48 hours with and without $20 \mu \mathrm{M}$ ManCou$\mathrm{H}$ media. Images captured at 20x magnification.

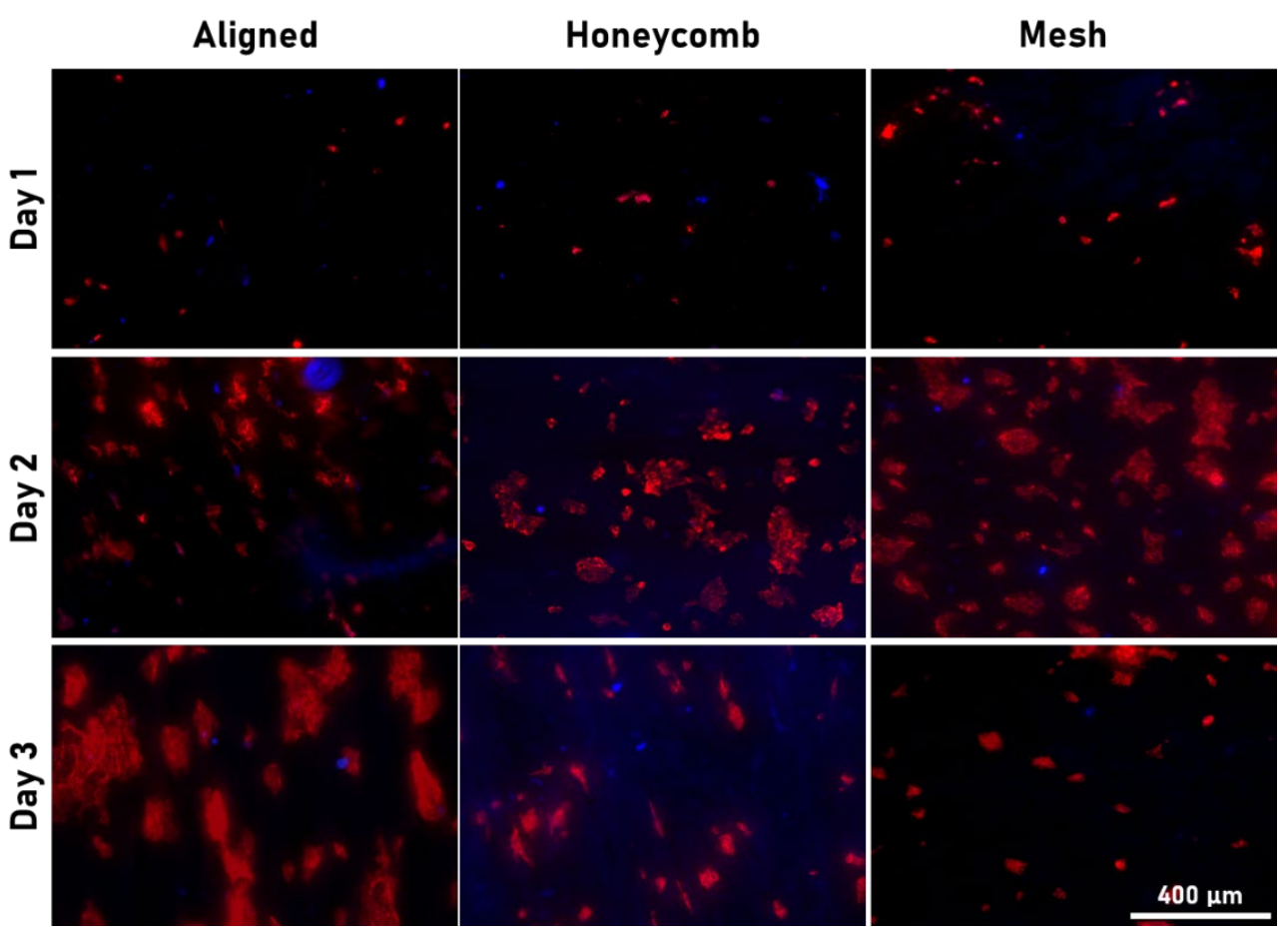

Figure S51. Fluorescent microscope images of normal breast epithelial cells (184B5) on different morphologies of PCL scaffold containing ManCou-H probe on days 1, 2 and 3 visualized through the DAPI (blue: ManCou-H) and TxRed (red: F-actin filaments) filter. Images captured at $10 \mathrm{X}$ magnification. 


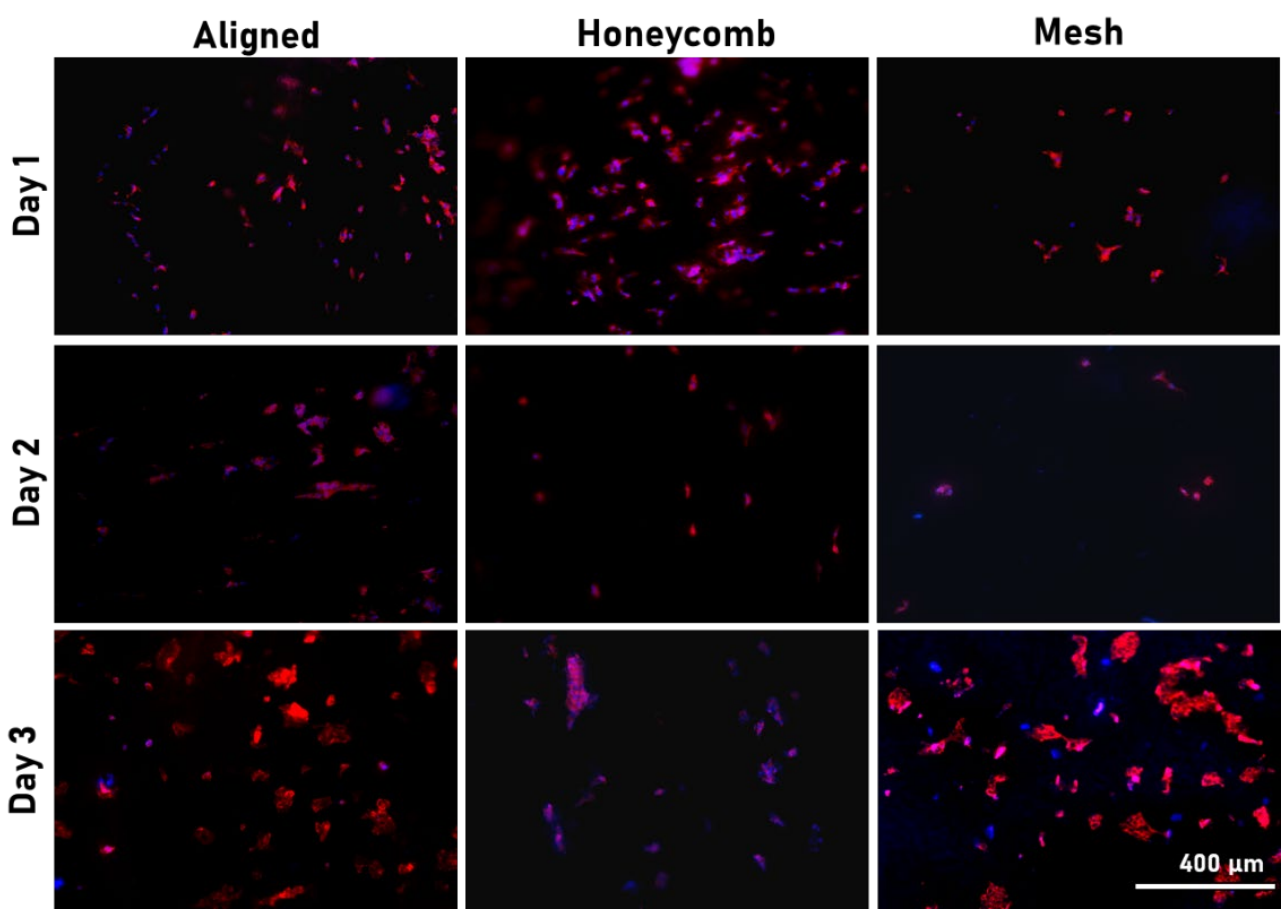

Figure S52. Fluorescent microscope images of normal breast epithelial cells (184B5) on different morphologies of PCL scaffolds on days 1,2 and 3 visualized through the DAPI (blue: nucleus) and TxRed (red: F-actin filaments) filter. Images captured at 10X magnification.

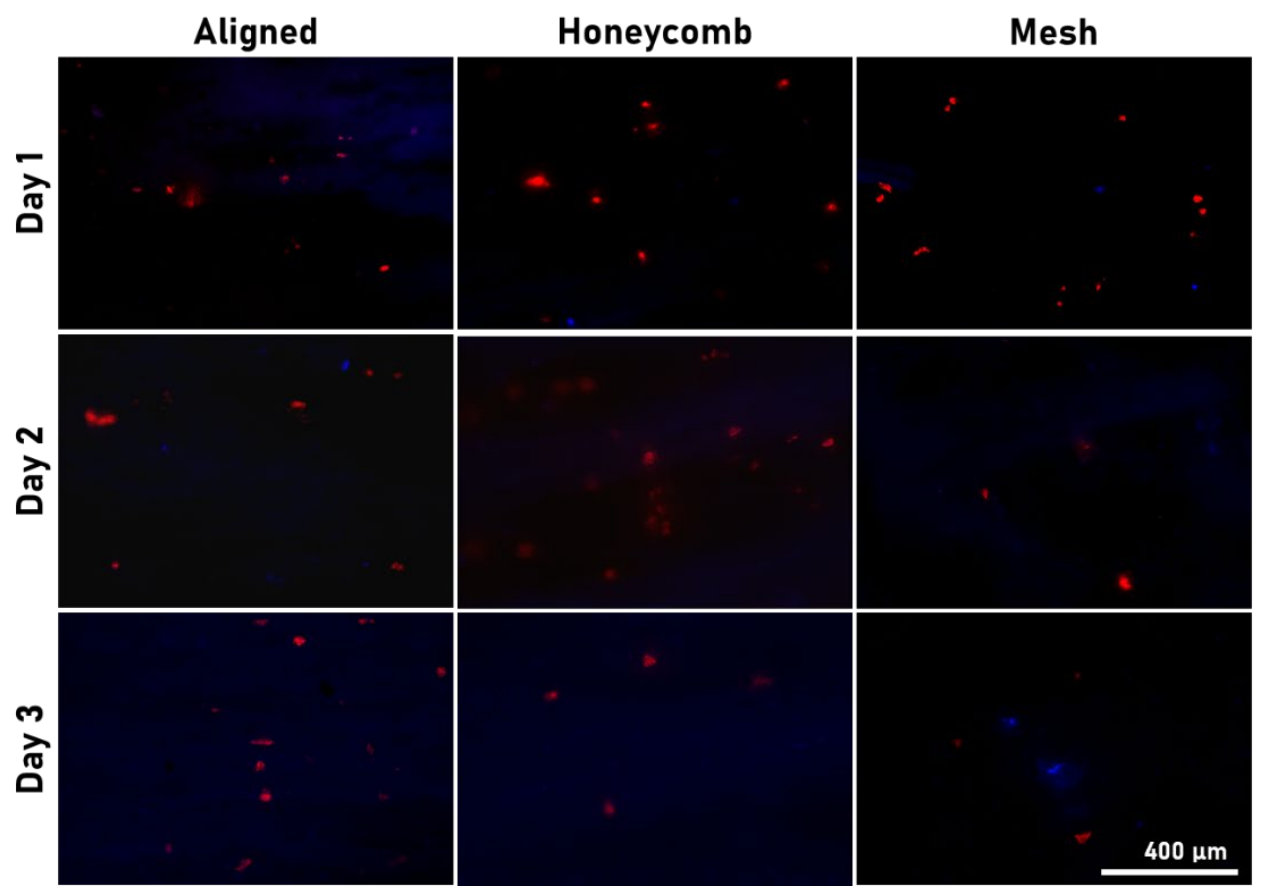


Figure S53. Fluorescent microscope images of human embryonic kidney epithelial cells (HEK-293) on different morphologies of PCL scaffold containing ManCou-H probe on days 1, 2 and 3 visualized through the DAPI (blue: ManCou-H) and TxRed (red: F-actin filaments) filter. Images captured at 10X magnification.

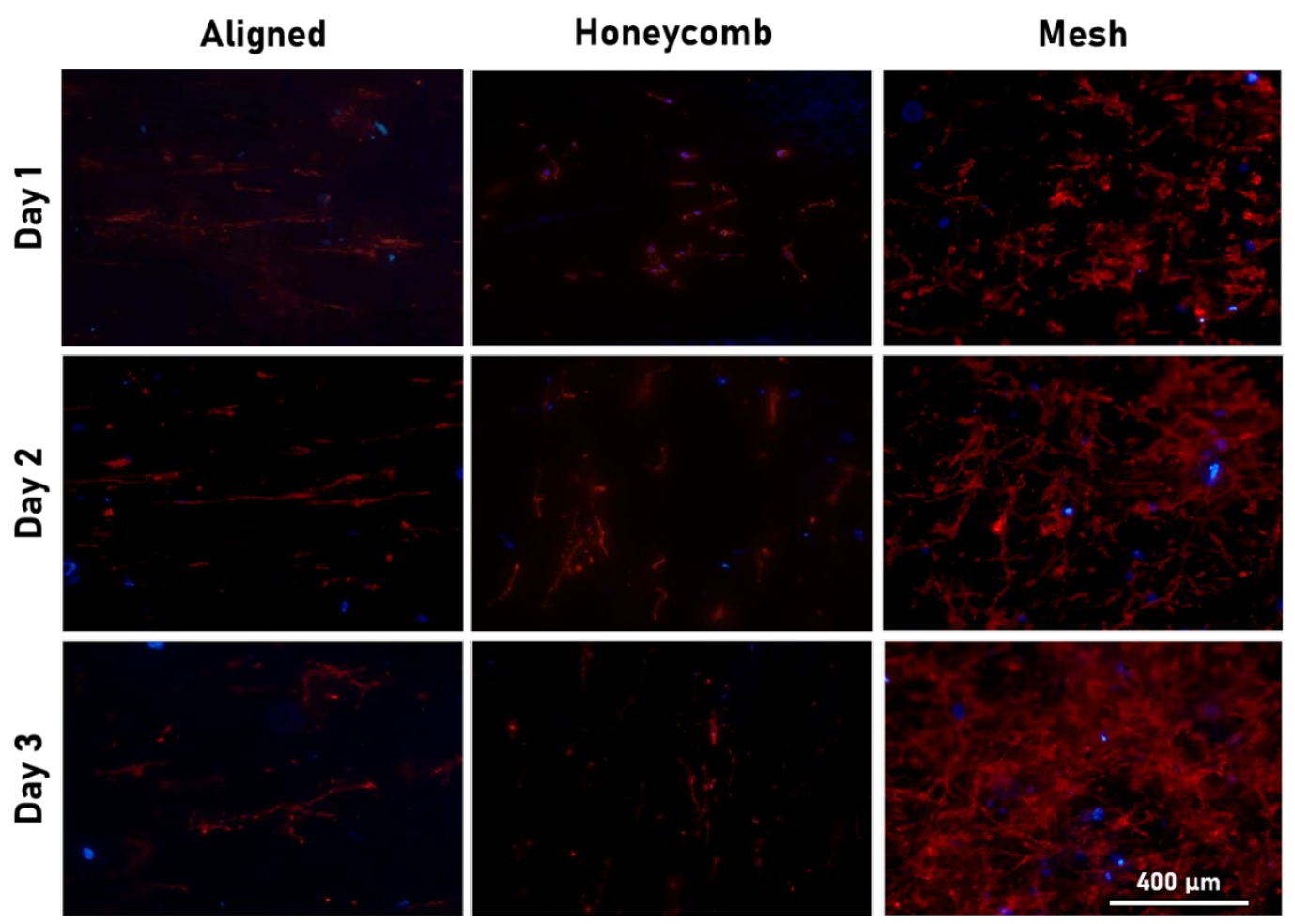

Figure S54. Fluorescent microscope images of human dermal fibroblasts cells (HDFa) on different morphologies of PCL scaffold containing ManCou-H probe on days 1, 2 and 3 visualized through the DAPI (blue: ManCou-H) and TxRed (red: F-actin filaments) filter. Images captured at $10 \mathrm{X}$ magnification. 


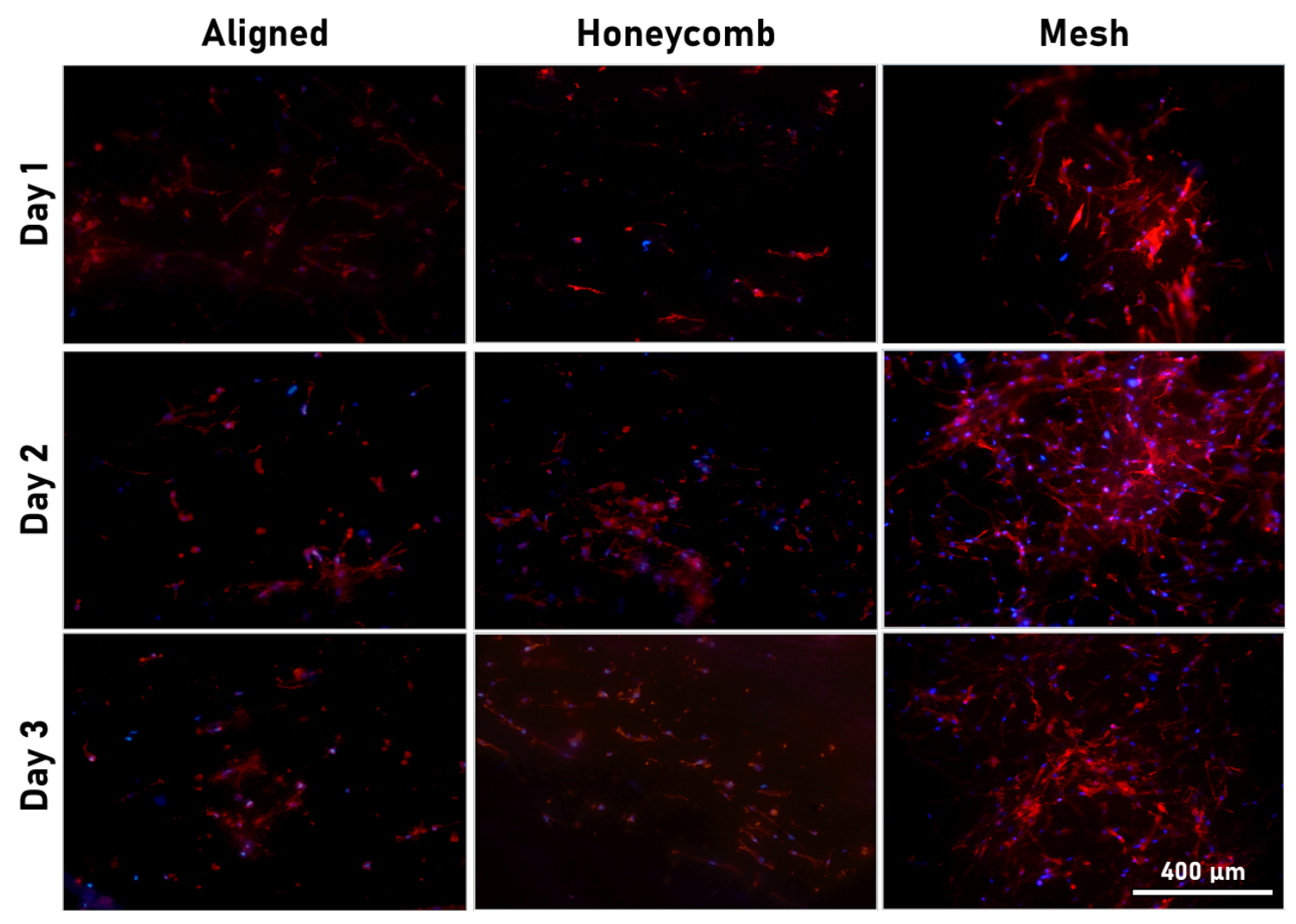

Figure S55. Fluorescent microscope images of human dermal fibroblasts (HDFa) on different morphologies of PCL scaffolds on days 1,2 and 3 visualized through the DAPI (blue: nucleus) and TxRed (red: F-actin filaments) filter. Images captured at $10 \mathrm{X}$ magnification. 


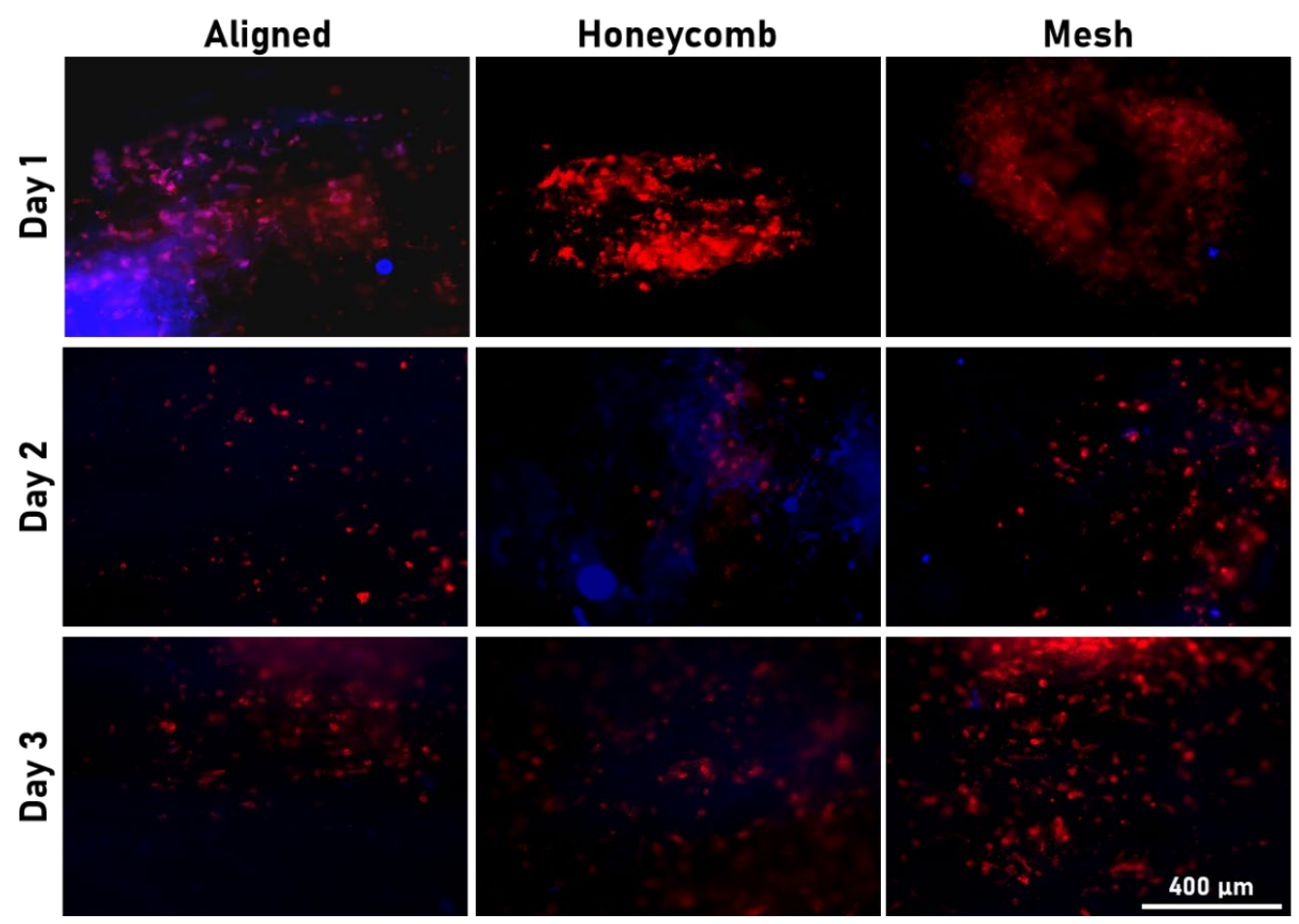

Figure S56. Fluorescent microscope images of renal adenocarcinoma cells (786-O) on different morphologies of PCL scaffold containing ManCou-H probe on days 1, 2 and 3 visualized through the DAPI (blue: ManCou-H) and TxRed (red: F-actin filaments) filter. Images captured at $10 \mathrm{X}$ magnification.

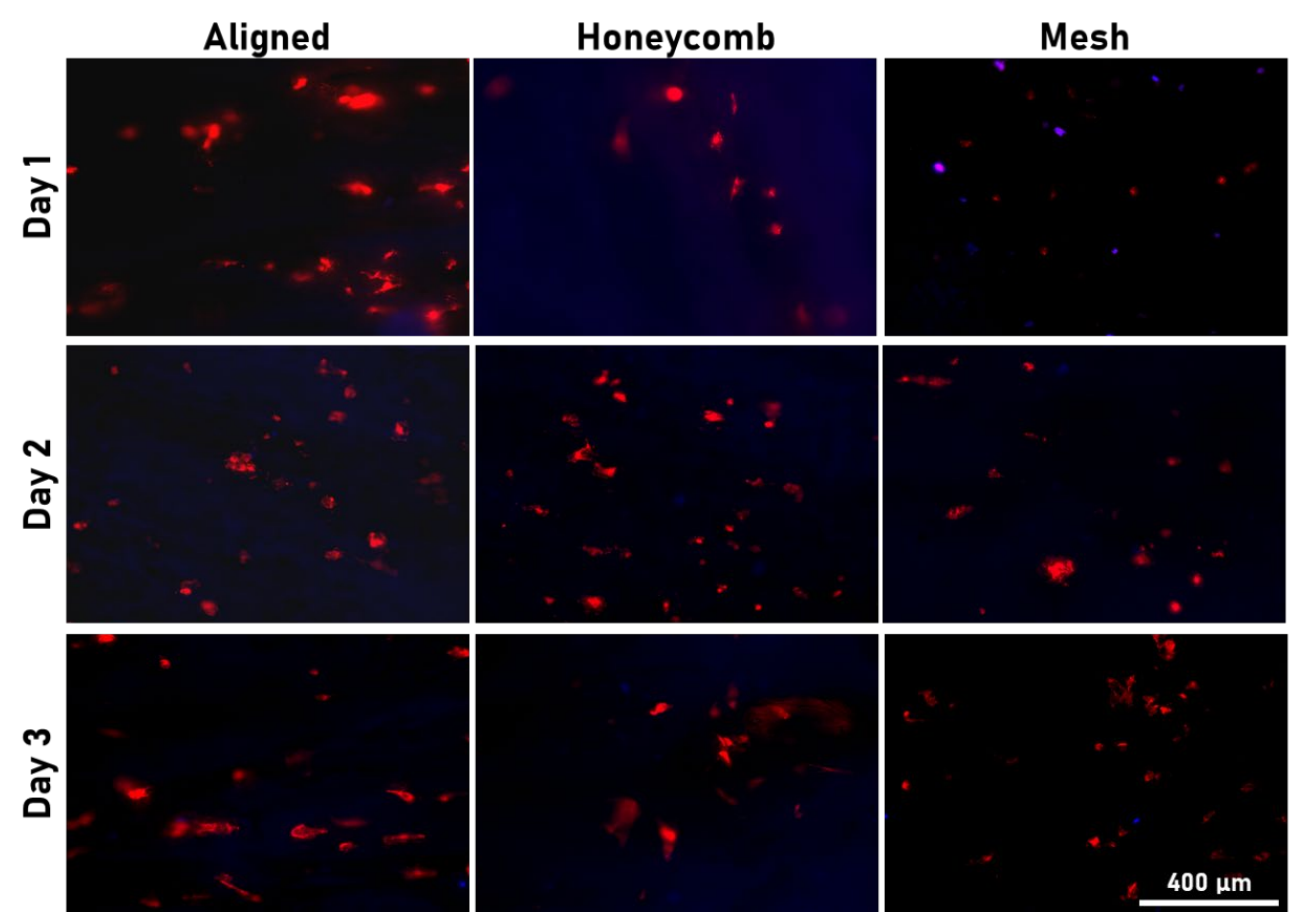


Figure S57. Fluorescent microscope images of clear cell renal carcinoma cells (Caki-1) on different morphologies of PCL scaffold containing ManCou-H probe on days 1, 2 and 3 visualized through the DAPI (blue: ManCou-H) and TxRed (red: F-actin filaments) filter. Images captured at $10 \mathrm{X}$ magnification.

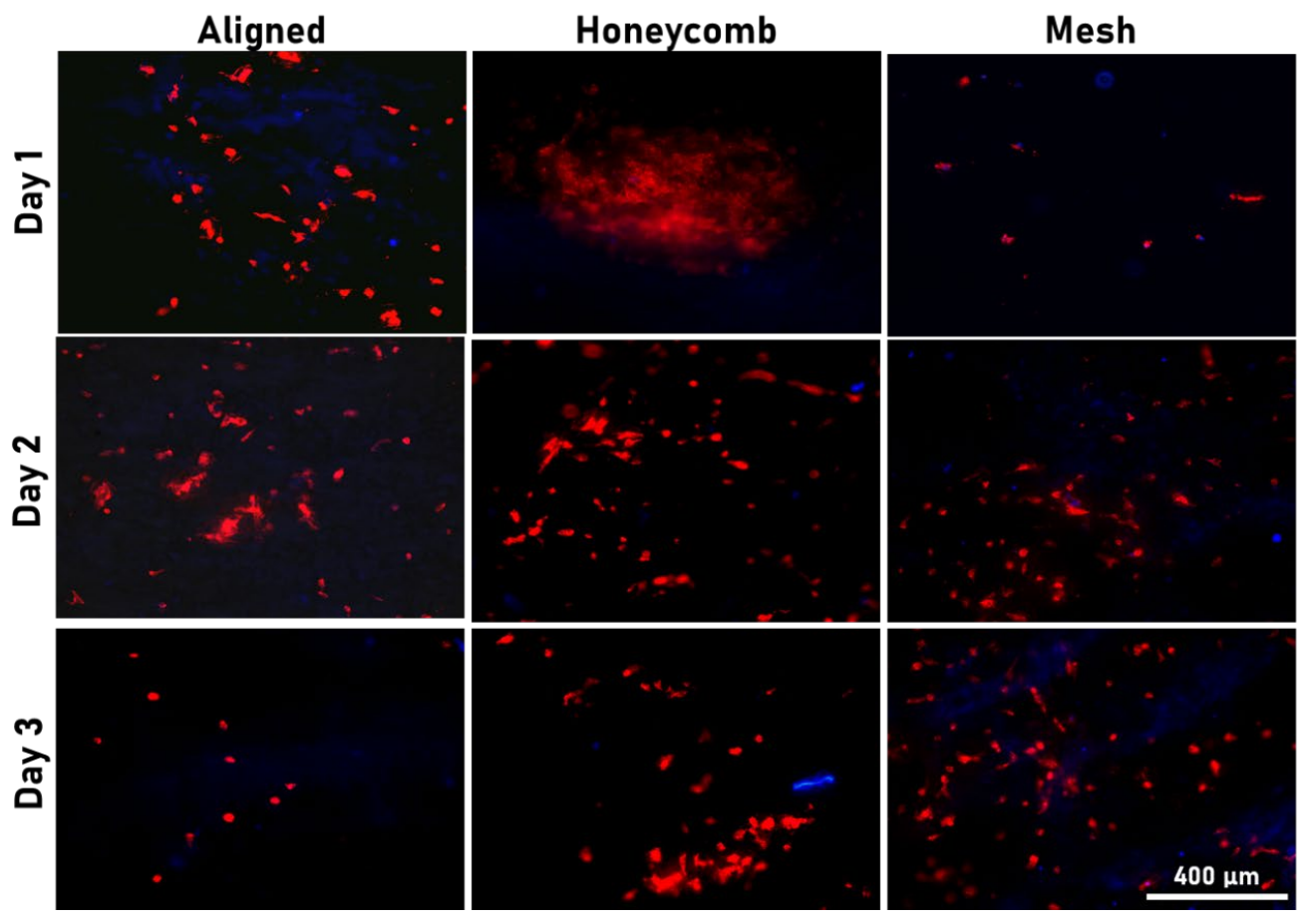

Figure S58. Fluorescent microscope images of renal adenocarcinoma cells established through pleural effusion (ACHN) on different morphologies of PCL scaffold containing ManCou-H probe on days 1, 2 and 3 visualized through the DAPI (blue: ManCou-H) and TxRed (red: F-actin filaments) filter. Images captured at 10X magnification. 


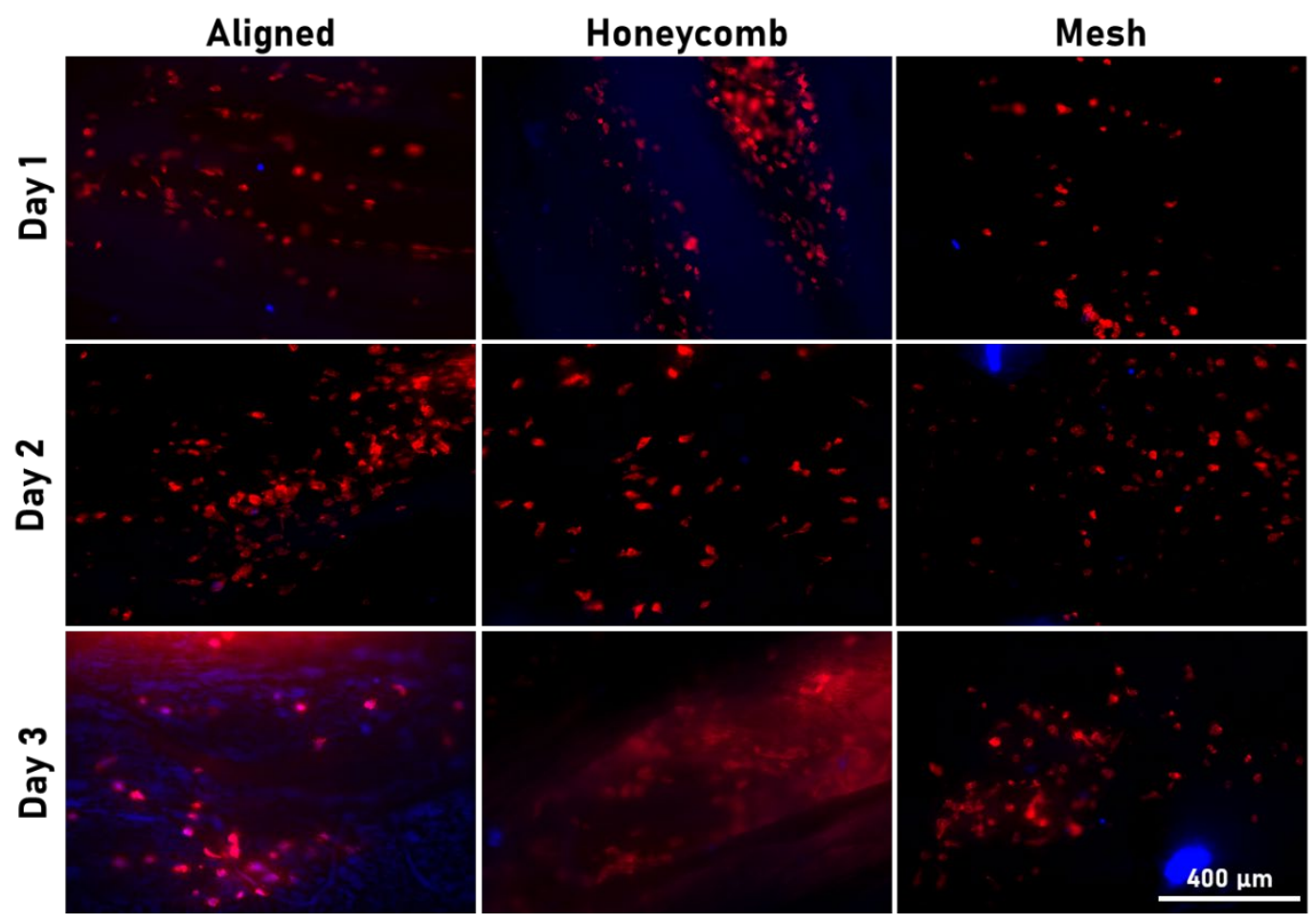

Figure S59. Fluorescent microscope images of lung carcinoma cells (A549) on different morphologies of PCL scaffold containing ManCou-H probe on days 1, 2 and 3 visualized through the DAPI (blue: ManCou-H) and TxRed (red: F-actin filaments) filter. Images captured at $10 \mathrm{X}$ magnification. 


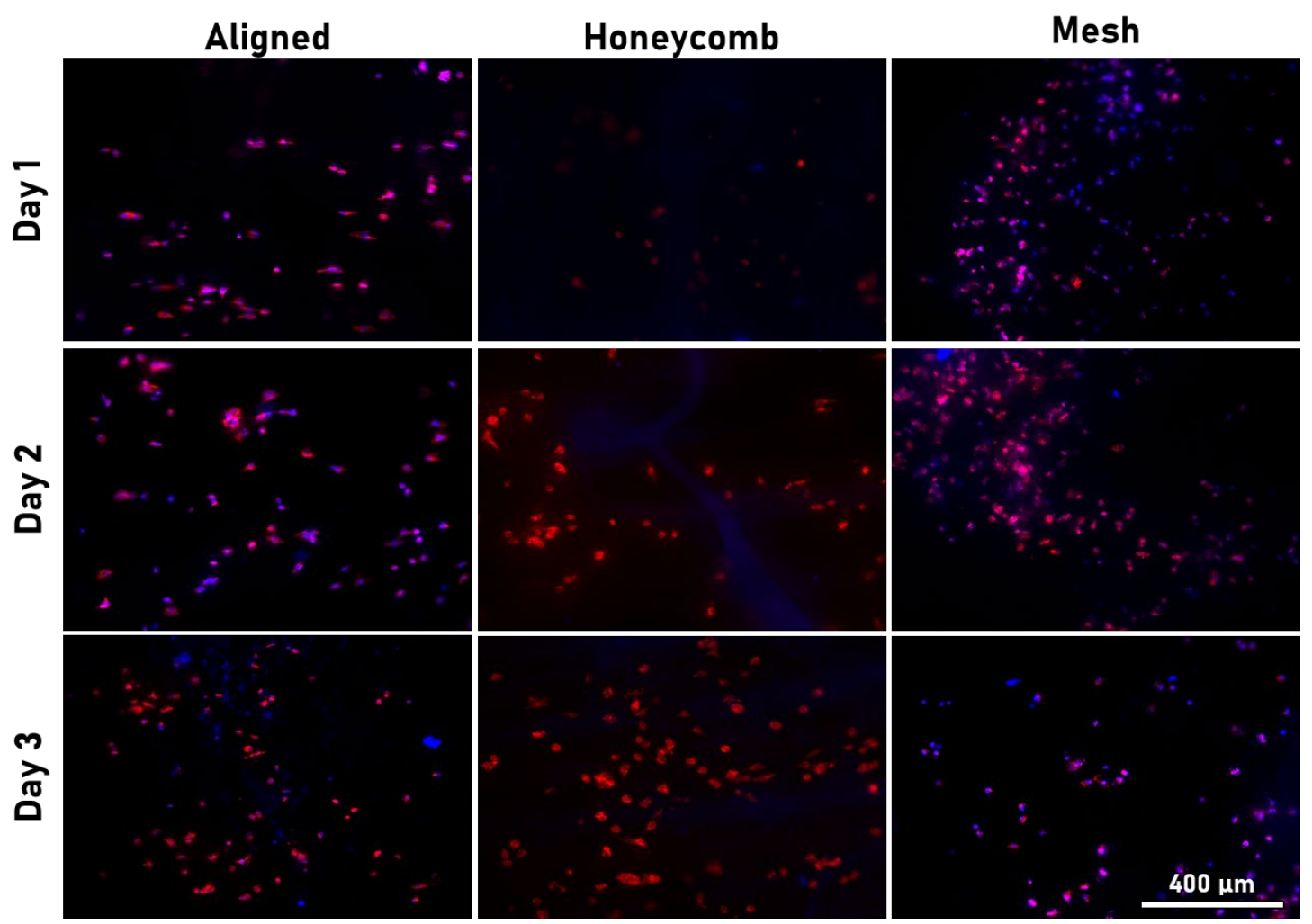

Figure S60. Fluorescent microscope images of breast adenocarcinoma cells (MCF7) on different morphologies of PCL scaffold containing ManCou-H probe on days 1, 2 and 3 visualized through the DAPI (blue: ManCou-H) and TxRed (red: F-actin filaments) filter. Images captured at 10X magnification. 


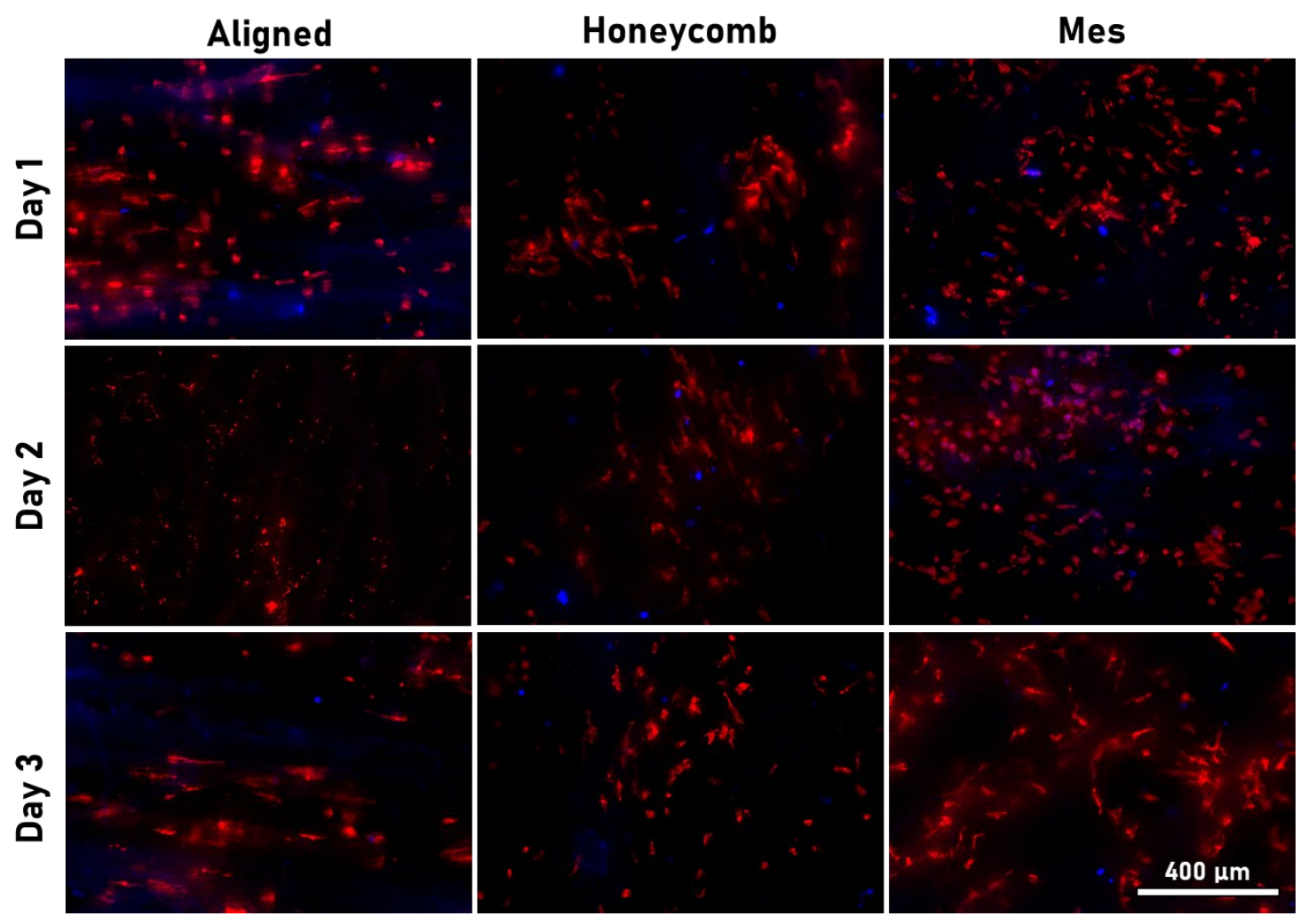

Figure S61. Fluorescent microscope images of triple negative breast cancer cells (MDAMB231) on different morphologies of PCL scaffold containing ManCou-H probe on days 1, 2 and 3 visualized through the DAPI (blue: ManCou-H) and TxRed (red: F-actin filaments) filter. Images captured at 10X magnification. 

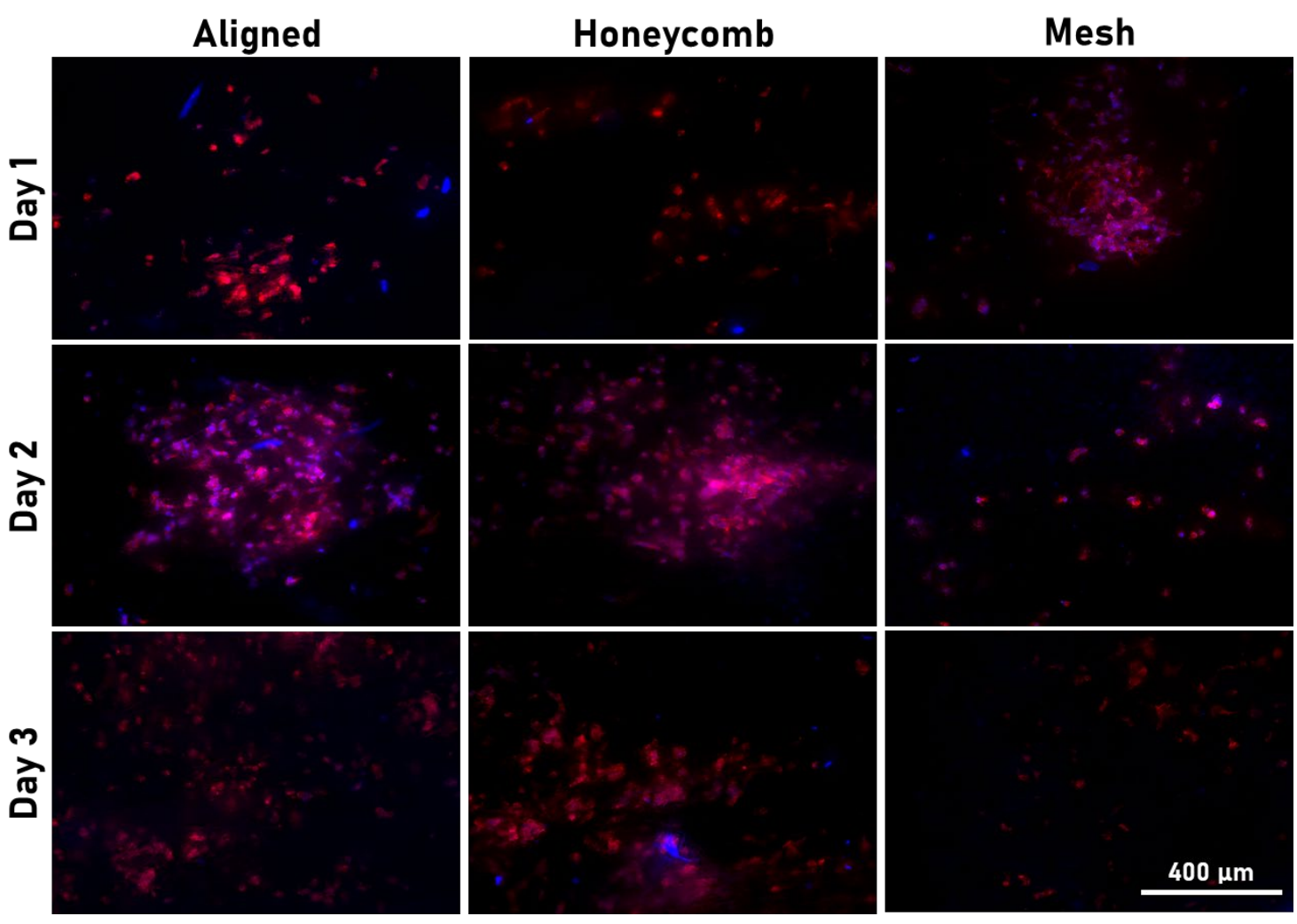

Figure S62. Fluorescent microscope images of premalignant cancer cells (MCF10AneoT) on different morphologies of PCL scaffold containing ManCou-H probe on days 1, 2 and 3 visualized through the DAPI (blue: ManCou-H) and TxRed (red: F-actin filaments) filter. Images captured at $10 \mathrm{X}$ magnification.

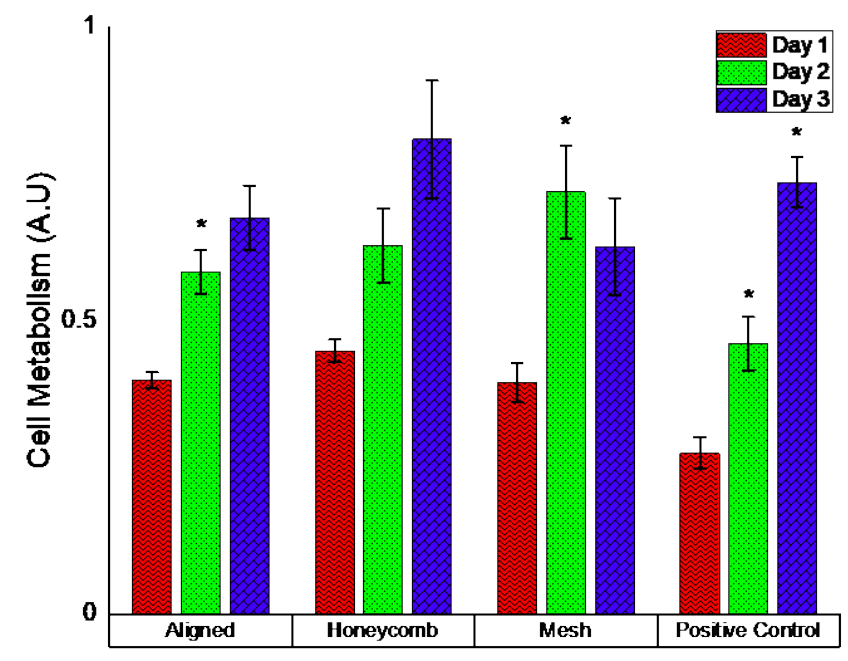


Figure S63. The cellular metabolism of 184B5 cells on scaffolds without probes on days 1,2 and 3. Significance established at $* \mathrm{p}<0.05$. 


\section{Conclusions}

In this dissertation, scaffolds of varying topographies, but with the same surface properties were fabricated to understand the role of biophysical cues on cells. One of the main contributions lies in the ability to electrospin these scaffolds of different structures without post-processing or using any templates from the same sol-gel. While the different topographies of the scaffolds affect the mechanical properties of the scaffolds, retaining the surface chemistry across the different scaffolds helps restrict variations in mechanical cues alone. The ability to electrospin these structures repeatedly and in a consistent manner, with control over the parameters helps provide reliability to the results - thus, addressing a challenge in this field. A novel self-assembly system was used for electrospinning PCLPANI honeycomb structures without the use of a template. Repeatability of results in obtaining nanostructures were proven even while using a multi-solvent, heterogenous polymer solution. This contribution helps to negate the previous concerns over repeatability and reliability of resultant nanostructures when using electrospinning and using provides flexibility in the choice of polymers and solvents used.

The overall focus was on the use of the scaffold system with varying topographies and associated mechanical properties but similar chemical properties to study the behavior of cells. Previous studies have focused on the use of scaffolds (hydrogels or electropsun networks) with different chemical gradients or the use of a 2D system, which are not the true representations of the in vivo environment. From an experimental point of view, this work contributes in eliminating a variable (differing chemical gradients) and developing a 3D system to understand the effects of biophysical cues. The current scaffold system can potentially be incorporated with drugs, growth factors or signaling molecules for delivery in a controlled manner, as demonstrated with the use of fluorescent GLUT-5 specific probes. The differences in cell behavior were also co-related with existing literature to understand the underlying signaling cascades involved or the nature of disease progression. The differences in protein expression, cell motility, cell viability and cellular infiltration were also analyzed to provide information to design better biomaterials and develop platforms to investigate the effects of biophysical cues in detail. This work is intended to guide future efforts in characterizing cellular responses in a $3 \mathrm{D}$ environment, map signaling pathways and tune the biochemical environment within the controlled biophysical environment created by the scaffolds. 


\section{Future Work}

The study presented here included fibroblasts and a few selected cell lines representative of breast, bladder, and renal cancer at various stages of cancer progression were used. Based on the results, it is clear that the cell viability and cell morphology is impacted by the substrate used. Hence, future studies involving different cell lines and scaffold designs, with accompanying analysis of protein expressions should be attempted. Possible direction of future work includes:

- The scaffolds presented here currently have a limited range of stiffness and morphologies. Future experiments can focus on the effects of topotaxis and durotaxis by fabricating scaffolds using the methods discussed in the dissertation. By having the topography or stiffness constant, the effects on the cells can be investigated.

- Future work needs to analyze the protein and gene expression changes in the cells during the time of study. It is essential to know the underlying pathways and relate it to existing literature.

- Additionally, the approach provides a novel platform for investigation of therapeutic targets in vitro.

- Longer time points of study need to be conducted in order to understand the longterm effects of the use of scaffolds. This will help understand the adaptability of cells and the reprogramming of cells.

- In vivo experiments using the scaffolds fabricated in chapter 3 will help to understand the effectiveness of the scaffolds as wound healing patches.

- The role of immune cells and fibroblasts in altering the tumor microenvironment is well known. Current co-cultures discussed in chapter 6 focus on the effect of scaffolds on epithelial cells and cancer cells. Future studies using fibroblasts and immune cells such as macrophages will help understand the effects of scaffold system on cells in an in-depth manner. The same when performed in vivo will help in the development of better in vivo models.

- Future experiments will seek to assess whether the preferences are based on cancer phenotypes classification such as undifferentiated, pre-malignant and metastatic. For example, the malignant and highly metastatic cell lines in breast, bladder and renal cells preferred the culture plate while the undifferentiated cells preferred the mesh scaffold. Adenocarcinomas preferred the aligned scaffolds as they require contact guidance. Hence, the next step in the study is to identify upregulation of mechanotransduction markers and EMT markers in these cell lines under these conditions. Tuning the scaffold used for the cells, will lead to optimized 3D cultures on scaffolds. 


\section{$9 \quad$ Reference List}

1. Eda, G., J. Liu, and S. Shivkumar, Solvent effects on jet evolution during electrospinning of semi-dilute polystyrene solutions. European Polymer Journal, 2007. 43(4): p. 1154-1167.

2. Guerrero, J., et al., Whipping of electrified liquid jets. Proceedings of the National Academy of Sciences, 2014. 111(38): p. 13763.

3. Wannatong, L., A. Sirivat, and P. Supaphol, Effects of solvents on electrospun polymeric fibers: preliminary study on polystyrene. Polymer International, 2004. 53(11): p. 1851-1859.

4. Reneker, D.H., et al., Nanofiber garlands of polycaprolactone by electrospinning. Polymer, 2002. 43(25): p. 6785-6794.

5. $\quad$ Pelipenko, J., et al., The impact of relative humidity during electrospinning on the morphology and mechanical properties of nanofibers. International Journal of Pharmaceutics, 2013. 456(1): p. 125-134.

6. Nezarati, R.M., M.B. Eifert, and E. Cosgriff-Hernandez, Effects of humidity and solution viscosity on electrospun fiber morphology. Tissue engineering. Part C, Methods, 2013. 19(10): p. 810-819.

7. Li, D. and Y.N. Xia, Electrospinning of nanofibers: Reinventing the wheel? Advanced Materials, 2004. 16(14): p. 1151-1170.

8. Theron, S.A., E. Zussman, and A.L. Yarin, Experimental investigation of the governing parameters in the electrospinning of polymer solutions. Polymer, 2004. 45(6): p. 2017-2030.

9. Deitzel, J.M., et al., The effect of processing variables on the morphology of electrospun nanofibers and textiles. Polymer, 2001. 42(1): p. 261-272.

10. Kim, K.W., et al., The effect of molecular weight and the linear velocity of drum surface on the properties of electrospun poly(ethylene terephthalate) nonwovens. Fibers and Polymers, 2004. 5(2): p. 122-127.

11. Baji, A., et al., Electrospinning of polymer nanofibers: Effects on oriented morphology, structures and tensile properties. Composites Science and Technology, 2010. 70(5): p. 703-718. 
12. Persano, L., et al., High performance piezoelectric devices based on aligned arrays of nanofibers of poly(vinylidenefluoride-co-trifluoroethylene). Nature Communications, 2013. 4: p. 1633.

13. Li, D., et al., Collecting Electrospun Nanofibers with Patterned Electrodes. Nano Letters, 2005. 5(5): p. 913-916.

14. Ding, Z., A. Salim, and B. Ziaie, Selective Nanofiber Deposition through FieldEnhanced Electrospinning. Langmuir, 2009. 25(17): p. 9648-9652.

15. Spivak, A.F., Y.A. Dzenis, and D.H. Reneker, A model of steady state jet in the electrospinning process. Mechanics Research Communications, 2000. 27(1): p. 37-42.

16. Yarin, A.L., S. Koombhongse, and D.H. Reneker, Bending instability in electrospinning of nanofibers. Journal of Applied Physics, 2001. 89(5): p. 3018-3026.

17. Hohman, M.M., et al., Electrospinning and electrically forced jets. II. Applications. Physics of Fluids, 2001. 13(8): p. 2221-2236.

18. Zhao, J., et al., Experimental and theoretical study on the electrospinning nanoporous fibers process. Materials Chemistry and Physics, 2016. 170: p. 294-302.

19. Yang, C., et al. Comparisons of fibers properties between vertical and horizontal type electrospinning systems. in Electrical Insulation and Dielectric Phenomena, 2009. CEIDP'09. IEEE Conference on. 2009. IEEE.

20. Loscertales, I.G., et al., Micro/Nano Encapsulation via Electrified Coaxial Liquid Jets. Science, 2002. 295(5560): p. 1695.

21. Bazilevsky, A.V., A.L. Yarin, and C.M. Megaridis, Co-electrospinning of Core-Shell Fibers Using a Single-Nozzle Technique. Langmuir, 2007. 23(5): p. 23112314.

22. Yarin, A.L., Coaxial electrospinning and emulsion electrospinning of core-shell fibers. 2011. 22(3): p. 310-317.

23. Xu, F., L. Li, and X. Cui, Fabrication of Aligned Side-by-Side TiO2/SnO2 Nanofibers via Dual-Opposite-Spinneret Electrospinning \%J Journal of Nanomaterials. 2012. 2012: p. 5.

24. $\quad \mathrm{Xu}, \mathrm{W}$. , et al., High-performance polyimide nanofibers reinforced polyimide nanocomposite films fabricated by co-electrospinning followed by hot-pressing. Journal of Applied Polymer Science, 2018. 135(47): p. 46849.

25. Weitz, R.T., et al., Polymer nanofibers via nozzle-free centrifugal spinning. Nano Letters, 2008. 8(4): p. 1187-1191. 
26. Sun, D., et al., Near-field electrospinning. Nano letters, 2006. 6(4): p. 839-842.

27. Simm, W., et al., Fibre fleece of electrostatically spun fibres and methods of making same. 1979, Google Patents.

28. Yu, M., et al., Recent Advances in Needleless Electrospinning of Ultrathin Fibers: From Academia to Industrial Production. Macromolecular Materials and Engineering, 2017. 302(7): p. 19.

29. $\mathrm{Xu}, \mathrm{X}$., et al., Preparation of Core-Sheath Composite Nanofibers by Emulsion Electrospinning. 2006. 27(19): p. 1637-1642.

30. Shin, S.R., et al., Graphene-based materials for tissue engineering. Advanced Drug Delivery Reviews, 2016. 105: p. 255-274.

31. Qi, H.X., et al., Encapsulation of drug reservoirs in fibers by emulsion electrospinning: Morphology characterization and preliminary release assessment. Biomacromolecules, 2006. 7(8): p. 2327-2330.

32. Yang, Y., et al., Promotion of skin regeneration in diabetic rats by electrospun core-sheath fibers loaded with basic fibroblast growth factor. Biomaterials, 2011. 32(18): p. 4243-4254.

33. Maretschek, S., A. Greiner, and T. Kissel, Electrospun biodegradable nanofiber nonwovens for controlled release of proteins. Journal of Controlled Release, 2008. 127(2): p. $180-187$.

34. $\mathrm{Xu}, \mathrm{X} . \mathrm{L}$. , et al., Ultrafine medicated fibers electrospun from $\mathrm{W} / \mathrm{O}$ emulsions. Journal of Controlled Release, 2005. 108(1): p. 33-42.

35. $\mathrm{Xu}, \mathrm{X} . \mathrm{L}$., et al., Ultrafine PEG-PLA fibers loaded with both paclitaxel and doxorubicin hydrochloride and their in vitro cytotoxicity. European Journal of Pharmaceutics and Biopharmaceutics, 2009. 72(1): p. 18-25.

36. Zhang, H., et al., Dual-delivery of VEGF and PDGF by double-layered electrospun membranes for blood vessel regeneration. Biomaterials, 2013. 34(9): p. 2202-2212.

37. Li, X.Q., et al., Encapsulation of proteins in poly (L-lactide-co-caprolactone) fibers by emulsion electrospinning. Colloids and Surfaces B-Biointerfaces, 2010. 75(2): p. 418424.

38. Su, Y., et al., Controlled release of dual drugs from emulsion electrospun nanofibrous mats. Colloids and Surfaces B-Biointerfaces, 2009. 73(2): p. 376-381.

39. Zhou, F., et al., Nanofiber-mediated microRNA-126 delivery to vascular endothelial cells for blood vessel regeneration. Acta Biomaterialia, 2016. 43: p. 303-313. 
40. Wang, Z.B., et al., Evaluation of emulsion electrospun polycaprolactone/hyaluronan/epidermal growth factor nanofibrous scaffolds for wound healing. Journal of Biomaterials Applications, 2016. 30(6): p. 686-698.

41. Nikmaram, N., et al., Emulsion-based systems for fabrication of electrospun nanofibers: food, pharmaceutical and biomedical applications. RSC Advances, 2017. 7(46): p. 28951-28964.

42. Chinnappan, A., et al., An overview of electrospun nanofibers and their application in energy storage, sensors and wearable/flexible electronics. Journal of Materials Chemistry C, 2017. 5(48): p. 12657-12673.

43. Timin, A.S., et al., Multifunctional Scaffolds with Improved Antimicrobial Properties and Osteogenicity Based on Piezoelectric Electrospun Fibers Decorated with Bioactive Composite Microcapsules. ACS Applied Materials \& Interfaces, 2018. 10(41): p. 34849-34868.

44. Perez, R.A. and H.-W. Kim, Core-shell designed scaffolds for drug delivery and tissue engineering. Acta Biomaterialia, 2015. 21: p. 2-19.

45. Subbiah, R. and R.E. Guldberg, Materials Science and Design Principles of Growth Factor Delivery Systems in Tissue Engineering and Regenerative Medicine. Advanced Healthcare Materials, 2019. 8(1): p. 1801000.

46. Whited, B.M. and M.N. Rylander, The influence of electrospun scaffold topography on endothelial cell morphology, alignment, and adhesion in response to fluid flow. Biotechnology and Bioengineering, 2014. 111(1): p. 184-195.

47. Ravichandran, R., et al., Effects of nanotopography on stem cell phenotypes. 2009. 1(1): p. 55.

48. Kim, D.H., et al., Nanoscale cues regulate the structure and function of macroscopic cardiac tissue constructs. Proceedings of the National Academy of Sciences of the United States of America, 2010. 107(2): p. 565-570.

49. Patel, S., et al., Bioactive nanofibers: Synergistic effects of nanotopography and chemical signaling on cell guidance. Nano Letters, 2007. 7(7): p. 2122-2128.

50. Christopherson, G.T., H. Song, and H.-Q. Mao, The influence of fiber diameter of electrospun substrates on neural stem cell differentiation and proliferation. Biomaterials, 2009. 30(4): p. 556-564.

51. Chew, S.Y., et al., The effect of the alignment of electrospun fibrous scaffolds on Schwann cell maturation. 2008. 29(6): p. 653-661. 
52. Ayres, C., et al., Modulation of anisotropy in electrospun tissue-engineering scaffolds: Analysis of fiber alignment by the fast Fourier transform. 2006. 27(32): p. 55245534.

53. Cheng, H.L., et al., Biomedical application and controlled drug release of electrospun fibrous materials. Materials Science \& Engineering C-Materials for Biological Applications, 2018. 90: p. 750-763.

54. Contreras-Cáceres, R., et al., Electrospun Nanofibers: Recent Applications in Drug Delivery and Cancer Therapy. Nanomaterials, 2019. 9(4).

55. Fullana, M.J. and G.E. Wnek, Electrospun collagen and its applications in regenerative medicine. Drug Delivery and Translational Research, 2012. 2(5): p. 313-322.

56. Sell, S.A., et al., The Use of Natural Polymers in Tissue Engineering: A Focus on Electrospun Extracellular Matrix Analogues. Polymers, 2010. 2(4): p. 522-553.

57. Campiglio, C.E., C. Marcolin, and L. Draghi, Electrospun ECM macromolecules as biomimetic scaffold for regenerative medicine: challenges for preserving conformation and bioactivity. Aims Materials Science, 2017. 4(3): p. 638-669.

58. Lee, K.Y., et al., Electrospinning of polysaccharides for regenerative medicine. Advanced Drug Delivery Reviews, 2009. 61(12): p. 1020-1032.

59. Sajkiewicz, P. and D. Kolbuk, Electrospinning of gelatin for tissue engineering molecular conformation as one of the overlooked problems. Journal of Biomaterials Science-Polymer Edition, 2014. 25(18): p. 2009-2022.

60. Qasim, S.B., et al., Electrospinning of Chitosan-Based Solutions for Tissue Engineering and Regenerative Medicine. International Journal of Molecular Sciences, 2018. 19(2): p. 26.

61. Kalantari, K., et al., Biomedical applications of chitosan electrospun nanofibers as a green polymer - Review. Carbohydrate Polymers, 2019. 207: p. 588-600.

62. Zhang, X.H., M.R. Reagan, and D.L. Kaplan, Electrospun silk biomaterial scaffolds for regenerative medicine. Advanced Drug Delivery Reviews, 2009. 61(12): p. 988-1006.

63. Zhang, J.G. and X.M. Mo, Current research on electrospinning of silk fibroin and its blends with natural and synthetic biodegradable polymers. Frontiers of Materials Science, 2013. 7(2): p. 129-142.

64. Majidi, S.S., et al., Wet electrospun alginate/gelatin hydrogel nanofibers for 3D cell culture. International Journal of Biological Macromolecules, 2018. 118: p. 1648-1654. 
65. Xie, J., et al., Osteogenic differentiation and bone regeneration of iPSC-MSCs supported by a biomimetic nanofibrous scaffold. Acta Biomaterialia, 2016. 29: p. 365-379.

66. Joy, J., et al., Gelatin - Oxidized carboxymethyl cellulose blend based tubular electrospun scaffold for vascular tissue engineering. International Journal of Biological Macromolecules, 2018. 107: p. 1922-1935.

67. Li, Z., et al., Solution fibre spinning technique for the fabrication of tuneable decellularised matrix-laden fibres and fibrous micromembranes. Acta Biomaterialia, 2018. 78: p. 111-122.

68. Pezeshki-Modaress, M., M. Zandi, and S. Rajabi, Tailoring the gelatin/chitosan electrospun scaffold for application in skin tissue engineering: an in vitro study. Progress in Biomaterials, 2018. 7(3): p. 207-218.

69. Chen, H., et al., Multiscale regeneration scaffold in vitro and in vivo. Journal of Biomedical Materials Research Part B: Applied Biomaterials, 2018. 106(3): p. 1218-1225.

70. Honarpardaz, A., et al., Enhanced chondrogenic differentiation of bone marrow mesenchymal stem cells on gelatin/glycosaminoglycan electrospun nanofibers with different amount of glycosaminoglycan. Journal of Biomedical Materials Research Part A, 2019. 107(1): p. 38-48.

71. Bian, T., et al., The construction and performance of multi-level hierarchical hydroxyapatite (HA)/collagen composite implant based on biomimetic bone Haversian motif. Materials \& Design, 2019. 162: p. 60-69.

72. Ko, E., et al., Electrospun Silk Fibroin Nanofibrous Scaffolds with Two-Stage Hydroxyapatite Functionalization for Enhancing the Osteogenic Differentiation of Human Adipose-Derived Mesenchymal Stem Cells. ACS Applied Materials \& Interfaces, 2018. 10(9): p. 7614-7625.

73. Deng, L., et al., Study on wettability, mechanical property and biocompatibility of electrospun gelatin/zein nanofibers cross-linked by glucose. Food Hydrocolloids, 2019. 87: p. 1-10.

74. Cipitria, A., et al., Design, fabrication and characterization of PCL electrospun scaffolds-a review. Journal of Materials Chemistry, 2011. 21(26): p. 9419-9453.

75. Woodruff, M.A. and D.W.J.P.i.p.s. Hutmacher, The return of a forgotten polymerPolycaprolactone in the 21st century. 2010. 35(10): p. 1217-1256.

76. Guo, B. and P.X.J.B. Ma, Conducting polymers for tissue engineering. 2018. 19(6): p. 1764-1782. 
77. Rajabi, A.H., M. Jaffe, and T.L. Arinzeh, Piezoelectric materials for tissue regeneration: a review. Acta biomaterialia, 2015. 24: p. 12-23.

78. Nagam Hanumantharao, S., C. Que, and S. Rao, Self-assembly of $3 D$ nanostructures in electrospun polycaprolactone-polyaniline fibers and their application as scaffolds for tissue engineering. Materialia, 2019. 6: p. 100296.

79. Malikmammadov, E., et al., PCL and PCL-based materials in biomedical applications. 2018. 29(7-9): p. 863-893.

80. Huang, Z.B., et al., Conducting polypyrrole in tissue engineering applications. Frontiers of Materials Science, 2014. 8(1): p. 39-45.

81. Bertuoli, P.T., et al., Electrospun Conducting and Biocompatible Uniaxial and Core-Shell Fibers Having Poly(lactic acid), Poly(ethylene glycol), and Polyaniline for Cardiac Tissue Engineering. ACS Omega, 2019. 4(2): p. 3660-3672.

82. de Castro, J.G., et al., Designing a novel nanocomposite for bone tissue engineering using electrospun conductive PBAT/polypyrrole as a scaffold to direct nanohydroxyapatite electrodeposition. RSC Advances, 2016. 6(39): p. 32615-32623.

83. Granato, A.E.C., et al., Polypyrrole increases branching and neurite extension by Neuro2A cells on PBAT ultrathin fibers. Nanomedicine: Nanotechnology, Biology and Medicine, 2018. 14(6): p. 1753-1763.

84. Wu, Y., V.D. Ranjan, and Y. Zhang, A Living 3D In Vitro Neuronal Network Cultured inside Hollow Electrospun Microfibers. Advanced Biosystems, 2018. 2(5): p. 1700218.

85. Wang, L., et al., Electrospun conductive nanofibrous scaffolds for engineering cardiac tissue and 3D bioactuators. Acta Biomaterialia, 2017. 59: p. 68-81.

86. Kharazi, A.Z., et al., A nanofibrous bilayered scaffold for tissue engineering of small-diameter blood vessels. 2018. 29(12): p. 3151-3158.

87. Browe, D. and J. Freeman, Optimizing C2C12 myoblast differentiation using polycaprolactone-polypyrrole copolymer scaffolds. 2019. 107(1): p. 220-231.

88. Sedláková, V., et al., Enhanced bioactivity of electrospun PCL and PLLA scaffolds blended with amino-phosphazene. Materials Letters, 2018. 228: p. 339-343.

89. Ding, Y., et al., Electrospun Polyhydroxybutyrate/Poly(E-caprolactone)/58S SolGel Bioactive Glass Hybrid Scaffolds with Highly Improved Osteogenic Potential for Bone Tissue Engineering. ACS Applied Materials \& Interfaces, 2016. 8(27): p. 17098-17108. 
90. $\mathrm{Hu}, \mathrm{S}$., et al., Thermally induced self-agglomeration $3 D$ scaffolds with BMP-2loaded core-shell fibers for enhanced osteogenic differentiation of rat adipose-derived stem cells. International journal of nanomedicine, 2018. 13: p. 4145-4155.

91. Farkhondehnia, H., M. Amani Tehran, and F. Zamani, Fabrication of Biocompatible PLGA/PCL/PANI Nanofibrous Scaffolds with Electrical Excitability. Fibers and Polymers, 2018. 19(9): p. 1813-1819.

92. Yao, Q., et al., Three dimensional electrospun PCL/PLA blend nanofibrous scaffolds with significantly improved stem cells osteogenic differentiation and cranial bone formation. Biomaterials, 2017. 115: p. 115-127.

93. Zhang, X.-F., et al., Laminin-modified and aligned poly(3-hydroxybutyrate-co-3hydroxyvalerate)/polyethylene oxide nanofibrous nerve conduits promote peripheral nerve regeneration. Journal of Tissue Engineering and Regenerative Medicine, 2018. 12(1): p. e627-e636.

94. Jiang, L., et al., Electrospun nanofibrous thermoplastic polyurethane/poly(glycerol sebacate) hybrid scaffolds for vocal fold tissue engineering applications. Materials Science and Engineering: C, 2019.94: p. 740-749.

95. Mi, H.-Y., et al., Fabrication of fibrous silica sponges by self-assembly electrospinning and their application in tissue engineering for three-dimensional tissue regeneration. Chemical Engineering Journal, 2018. 331: p. 652-662.

96. Seethalakshmi, K., et al., 6-O-tritylchitosan reinforced polycaprolactone nano scaffolds for bone replacement applications-A physicochemical study. 2019.

97. $\mathrm{Hu}, \mathrm{W} .-\mathrm{W} .$, C.-H. Lin, and Z.-J. Hong, The enrichment of cancer stem cells using composite alginate/polycaprolactone nanofibers. Carbohydrate Polymers, 2019. 206: p. 70-79.

98. Hou, J., et al., Biomimetic Growth of Hydroxyapatite on Electrospun CA/PVP Core-Shell Nanofiber Membranes. Polymers, 2018. 10(9).

99. Sharifi, F., et al., Polycaprolactone/carboxymethyl chitosan nanofibrous scaffolds for bone tissue engineering application. International Journal of Biological Macromolecules, 2018. 115: p. 243-248.

100. Shrestha, B.K., et al., Development of polyamide-6,6/chitosan electrospun hybrid nanofibrous scaffolds for tissue engineering application. Carbohydrate Polymers, 2016. 148: p. 107-114.

101. Sadeghi, D., et al., Electrospun poly(hydroxybutyrate)/chitosan blend fibrous scaffolds for cartilage tissue engineering. Journal of Applied Polymer Science, 2016. 133(47): p. 9. 
102. Agrawal, P. and K. Pramanik, Chitosan-poly(vinyl alcohol) nanofibers by free surface electrospinning for tissue engineering applications. Tissue Engineering and Regenerative Medicine, 2016. 13(5): p. 485-497.

103. Li, H., et al., A facile method for fabricating nano/microfibrous three-dimensional scaffold with hierarchically porous to enhance cell infiltration. Journal of Applied Polymer Science, 2019. 136(3): p. 47046.

104. Fadaie, M., et al., Incorporation of nanofibrillated chitosan into electrospun PCL nanofibers makes scaffolds with enhanced mechanical and biological properties. Carbohydrate Polymers, 2018. 199: p. 628-640.

105. Huang, M., et al., Preparation of CS/PVA Nanofibrous Membrane with Tunable Mechanical Properties for Tympanic Member Repair. Macromolecular Research, 2018. 26(10): p. 892-899.

106. Thomas, M.S., et al., Electrospun polylactic acid-chitosan composite: a bio-based alternative for inorganic composites for advanced application. Journal of Materials Science: Materials in Medicine, 2018. 29(9): p. 137.

107. Chanda, A., et al., Electrospun chitosan/polycaprolactone-hyaluronic acid bilayered scaffold for potential wound healing applications. International Journal of Biological Macromolecules, 2018. 116: p. 774-785.

108. Mohamadi, F., et al., Electrospun nerve guide scaffold of poly( $\varepsilon$ caprolactone)/collagen/nanobioglass: an in vitro study in peripheral nerve tissue engineering. 2017. 105(7): p. 1960-1972.

109. Kang, Y., et al., Multilevel structural stereocomplex polylactic acid/collagen membranes by pattern electrospinning for tissue engineering. Polymer, 2018. 156: p. 250260.

110. Park, H.K., et al., Collagen/poly(d,l-lactic-co-glycolic acid) composite fibrous scaffold prepared by independent nozzle control multi-electrospinning apparatus for dura repair. Journal of Industrial and Engineering Chemistry, 2018. 66: p. 430-437.

111. In Kim, J. and C.S. Kim, Harnessing nanotopography of PCL/collagen nanocomposite membrane and changes in cell morphology coordinated with wound healing activity. Materials Science and Engineering: C, 2018. 91: p. 824-837.

112. Middleton, R., et al., Near-Field Electrospinning Patterning Polycaprolactone and Polycaprolactone/Collagen Interconnected Fiber Membrane. Macromolecular Materials and Engineering, 2018. 303(2): p. 1700463.

113. Gao, S., et al., An electrospun fiber reinforced scaffold promotes total meniscus regeneration in rabbit meniscectomy model. Acta Biomaterialia, 2018. 73: p. 127-140. 
114. Gao, S., et al., Fabrication and characterization of electrospun nanofibers composed of decellularized meniscus extracellular matrix and polycaprolactone for meniscus tissue engineering. Journal of Materials Chemistry B, 2017. 5(12): p. 2273-2285.

115. Chen, Y., et al., Hybrid scaffolds of $M g$ alloy mesh reinforced polymer/extracellular matrix composite for critical-sized calvarial defect reconstruction. Journal of Tissue Engineering and Regenerative Medicine, 2018. 12(6): p. 1374-1388.

116. Saadatkish, N., et al., A ternary nanofibrous scaffold potential for central nerve system tissue engineering. Journal of Biomedical Materials Research Part A, 2018. 106(9): p. 2394-2401.

117. Hu, J., et al., Electrospinning of poly(glycerol sebacate)-based nanofibers for nerve tissue engineering. Materials Science and Engineering: C, 2017. 70: p. 1089-1094.

118. Jiang, Y.-C., et al., Electrospun polycaprolactone/gelatin composites with enhanced cell-matrix interactions as blood vessel endothelial layer scaffolds. Materials Science and Engineering: C, 2017. 71: p. 901-908.

119. Kook, Y.-M., et al., Promotion of Vascular Morphogenesis of Endothelial Cells Co-Cultured with Human Adipose-Derived Mesenchymal Stem Cells Using Polycaprolactone/Gelatin Nanofibrous Scaffolds. Nanomaterials, 2018. 8(2).

120. Yu, K., et al., Fabrication of poly(ester-urethane)urea elastomer/gelatin electrospun nanofibrous membranes for potential applications in skin tissue engineering. RSC Advances, 2016. 6(77): p. 73636-73644.

121. Ma, J., et al., A novel electrospun-aligned nanoyarn/three-dimensional porous nanofibrous hybrid scaffold for annulus fibrosus tissue engineering. International journal of nanomedicine, 2018. 13: p. 1553-1567.

122. Baghersad, S., et al., Development of biodegradable electrospun gelatin/aloevera/poly(E-caprolactone) hybrid nanofibrous scaffold for application as skin substitutes. Materials Science and Engineering: C, 2018. 93: p. 367-379.

123. Saravani, S., M. Ebrahimian-Hosseinabadi, and D. Mohebbi-Kalhori, Polyglycerol sebacate/chitosan/gelatin nano-composite scaffolds for engineering neural construct. Materials Chemistry and Physics, 2019. 222: p. 147-151.

124. Sadeghi, A., M. Pezeshki-Modaress, and M. Zandi, Electrospun polyvinyl alcohol/gelatin/chondroitin sulfate nanofibrous scaffold: Fabrication and in vitro evaluation. International Journal of Biological Macromolecules, 2018. 114: p. 1248-1256.

125. Ye, K., et al., Three-dimensional electrospun nanofibrous scaffolds displaying bone morphogenetic protein-2-derived peptides for the promotion of osteogenic differentiation 
of stem cells and bone regeneration. Journal of Colloid and Interface Science, 2019. 534: p. 625-636.

126. Chen, W., et al., Superabsorbent 3D Scaffold Based on Electrospun Nanofibers for Cartilage Tissue Engineering. ACS Applied Materials \& Interfaces, 2016. 8(37): p. 2441524425.

127. Zhao, Q., et al., Regulation Effects of Biomimetic Hybrid Scaffolds on Vascular Endothelium Remodeling. ACS Applied Materials \& Interfaces, 2018. 10(28): p. 2358323594.

128. Zhao, Q., et al., Programmed Shape-Morphing Scaffolds Enabling Facile 3D Endothelialization. 2018. 28(29): p. 1801027.

129. Garcia Garcia, A., et al., Poly(E-caprolactone)/Hydroxyapatite 3D Honeycomb Scaffolds for a Cellular Microenvironment Adapted to Maxillofacial Bone Reconstruction. ACS Biomaterials Science \& Engineering, 2018. 4(9): p. 3317-3326.

130. Prabha, R.D., et al., Bioactive nano-fibrous scaffold for vascularized craniofacial bone regeneration. Journal of Tissue Engineering and Regenerative Medicine, 2018. 12(3): p. e1537-e1548.

131. Tiwari, A.P., et al., Bimodal fibrous structures for tissue engineering: Fabrication, characterization and in vitro biocompatibility. Journal of Colloid and Interface Science, 2016. 476: p. 29-34.

132. Grant, R., et al., Blended electrospinning with human liver extracellular matrix for engineering new hepatic microenvironments. Scientific Reports, 2019. 9(1): p. 6293.

133. Kim, J.Y., et al., Design of a modified electrospinning for the in-situ fabrication of $3 D$ cotton-like collagen fiber bundle mimetic scaffold. Materials Letters, 2019. 236: p. 521525.

134. Song, S.J., et al., Aligned laminin core-polydioxanone/collagen shell fiber matrices effective for neuritogenesis. Scientific Reports, 2018. 8: p. 11.

135. Liu, X., et al., Lecithin doped electrospun poly(lactic acid)-thermoplastic polyurethane fibers for hepatocyte viability improvement. Colloids and Surfaces B: Biointerfaces, 2019. 175: p. 264-271.

136. Wang, J., et al., Engineering PCL/lignin nanofibers as an antioxidant scaffold for the growth of neuron and Schwann cell. Colloids and Surfaces B: Biointerfaces, 2018. 169: p. 356-365.

137. Jaganathan, S.K., et al., Fabrication and characterisation of nanofibrous polyurethane scaffold incorporated with corn and neem oil using single stage 
electrospinning technique for bone tissue engineering applications. Journal of Polymer Research, 2018. 25(7): p. 146.

138. Didekhani, R., et al., Electrospun composite PLLA/Oyster shell scaffold enhances proliferation and osteogenic differentiation of stem cells. Biologicals, 2018. 54: p. 33-38.

139. Du, J., et al., Potential applications of three-dimensional structure of silk fibroin/poly(ester-urethane) urea nanofibrous scaffold in heart valve tissue engineering. Applied Surface Science, 2018. 447: p. 269-278.

140. Zhu, C.H., et al., Wrinkle-Free, Sandwich, Electrospun PLGA/SF Nanofibrous Scaffold for Skin Tissue Engineering. Ieee Transactions on Nanotechnology, 2018. 17(4): p. 675-679.

141. Yi, B., et al., Fabrication of high performance silk fibroin fibers via stable jet electrospinning for potential use in anisotropic tissue regeneration. Journal of Materials Chemistry B, 2018. 6(23): p. 3934-3945.

142. Luo, J., et al., 3-D mineralized silk fibroin/polycaprolactone composite scaffold modified with polyglutamate conjugated with BMP-2 peptide for bone tissue engineering. Colloids and Surfaces B: Biointerfaces, 2018. 163: p. 369-378.

143. Cai, J., et al., Dual-layer aligned-random nanofibrous scaffolds for improving gradient microstructure of tendon-to-bone healing in a rabbit extra-articular model. International journal of nanomedicine, 2018. 13: p. 3481-3492.

144. Yao, Q.K., et al., A novel application of electrospun silk fibroin/poly(l-lactic acidco-epsilon-caprolactone) scaffolds for conjunctiva reconstruction. Rsc Advances, 2018. 8(33): p. 18372-18380.

145. Serôdio, R., et al., Ultrasound sonication prior to electrospinning tailors silk fibroin/PEO membranes for periodontal regeneration. Materials Science and Engineering: C, 2019. 98: p. 969-981.

146. Cheng, G., et al., Incorporating platelet-rich plasma into coaxial electrospun nanofibers for bone tissue engineering. International Journal of Pharmaceutics, 2018. 547(1): p. 656-666.

147. Waghmare, V.S., et al., Starch based nanofibrous scaffolds for wound healing applications. Bioactive Materials, 2018. 3(3): p. 255-266.

148. Jaganathan, S.K., et al., Single stage electrospun multicomponent scaffold for bone tissue engineering application. Polymer Testing, 2018. 70: p. 244-254.

149. He, J., et al., Continuous twisted nanofiber yarns fabricated by double conjugate electrospinning. 2013. 14(11): p. 1857-1863. 
150. Gao, Y., et al., Biomineralized poly (l-lactic-co-glycolic acid)-tussah silk fibroin nanofiber fabric with hierarchical architecture as a scaffold for bone tissue engineering. Materials Science and Engineering: C, 2018. 84: p. 195-207.

151. Jaganathan, S.K., et al., Production and hemocompatibility assessment of novel electrospun polyurethane nanofibers loaded with dietary virgin coconut oil for vascular graft applications. Journal of Bioactive and Compatible Polymers, 2017. 33(2): p. 210223.

152. Pedram Rad, Z., J. Mokhtari, and M. Abbasi, Fabrication and characterization of PCL/zein/gum arabic electrospun nanocomposite scaffold for skin tissue engineering. Materials Science and Engineering: C, 2018. 93: p. 356-366.

153. Zakaria, S.M., et al., Nanophase Hydroxyapatite as a Biomaterial in Advanced Hard Tissue Engineering: A Review. Tissue Engineering Part B-Reviews, 2013. 19(5): p. 431-441.

154. Ito, A. and M. Kamihira, Tissue Engineering Using Magnetite Nanoparticles, in Nanoparticles in Translational Science and Medicine, A. Villaverde, Editor. 2011, Elsevier Academic Press Inc: San Diego. p. 355-395.

155. Cardoso, V.F., et al., Advances in Magnetic Nanoparticles for Biomedical Applications. Advanced Healthcare Materials, 2018. 7(5): p. 35.

156. Sedghi, R., et al., Novel biocompatible zinc-curcumin loaded coaxial nanofibers for bone tissue engineering application. Polymer, 2018. 142: p. 244-255.

157. Xu, Z., et al., Fabrication and biocompatibility of agarose acetate nanofibrous membrane by electrospinning. Carbohydrate Polymers, 2018. 197: p. 237-245.

158. Golafshan, N., M. Kharaziha, and M. Fathi, Tough and conductive hybrid graphene-PVA: Alginate fibrous scaffolds for engineering neural construct. Carbon, 2017. 111: p. 752-763.

159. Shrestha, S., et al., Electrodeless coating polypyrrole on chitosan grafted polyurethane with functionalized multiwall carbon nanotubes electrospun scaffold for nerve tissue engineering. Carbon, 2018. 136: p. 430-443.

160. Mahmoudi, N. and A. Simchi, On the biological performance of graphene oxidemodified chitosan/polyvinyl pyrrolidone nanocomposite membranes: In vitro and in vivo effects of graphene oxide. Materials Science and Engineering: C, 2017. 70: p. 121-131.

161. Nasajpour, A., et al., A Multifunctional Polymeric Periodontal Membrane with Osteogenic and Antibacterial Characteristics. Advanced Functional Materials, 2018. 28(3): p. 1703437. 
162. Li, H., et al., An electrospun poly(E-caprolactone) nanocomposite fibrous mat with a high content of hydroxyapatite to promote cell infiltration. RSC Advances, 2018. 8(44): p. 25228-25235.

163. Wu, G., et al., Enhanced biological properties of biomimetic apatite fabricated polycaprolactone/chitosan nanofibrous bio-composite for tendon and ligament regeneration. Journal of Photochemistry and Photobiology B: Biology, 2018. 178: p. 2732.

164. Pavliňáková, V., et al., Effect of halloysite nanotube structure on physical, chemical, structural and biological properties of elastic polycaprolactone/gelatin nanofibers for wound healing applications. Materials Science and Engineering: C, 2018. 91: p. 94-102.

165. Guo, F., et al., Fabrication of LaCl3-containing nanofiber scaffolds and their application in skin wound healing. Journal of Applied Polymer Science, 2018. 135(36): p. 46672.

166. Ezati, M., et al., Development of a PCL/gelatin/chitosan/ $\beta$-TCP electrospun composite for guided bone regeneration. Progress in Biomaterials, 2018. 7(3): p. 225-237.

167. Thompson, Z., et al., Fabrication and Characterization of Magnesium FerriteBased PCL/Aloe Vera Nanofibers. 2017. 10(8): p. 937.

168. Rijal, N.P., et al., Magnesium oxide-poly(E-caprolactone)-chitosan-based composite nanofiber for tissue engineering applications. Materials Science and Engineering: B, 2018. 228: p. 18-27.

169. Stone, H., S. Lin, and K. Mequanint, Preparation and characterization of electrospun rGO-poly(ester amide) conductive scaffolds. Materials Science and Engineering: C, 2019. 98: p. 324-332.

170. Gorodzha, S.N., et al., A comparison study between electrospun polycaprolactone and piezoelectric poly(3-hydroxybutyrate-co-3-hydroxyvalerate) scaffolds for bone tissue engineering. Colloids and Surfaces B: Biointerfaces, 2017. 160: p. 48-59.

171. Ramesh, S., et al., Fabrication and evaluation of poly(lactic acid), chitosan, and tricalcium phosphate biocomposites for guided bone regeneration. Journal of Applied Polymer Science, 2018. 135(39): p. 46692.

172. Sabzi, M., et al., Designing triple-shape memory polymers from a miscible polymer pair through dual-electrospinning technique. Journal of Applied Polymer Science, 2019. 136(19): p. 47471. 
173. Shin, Y.C., et al., Stimulated myoblast differentiation on graphene oxideimpregnated PLGA-collagen hybrid fibre matrices. Journal of Nanobiotechnology, 2015. 13(1): p. 21.

174. Yang, X., et al., Incorporation of silica nanoparticles to PLGA electrospun fibers for osteogenic differentiation of human osteoblast-like cells. Regenerative Biomaterials, 2018. 5(4): p. 229-238.

175. Lai, W.-Y., et al., In Vivo Investigation into Effectiveness of Fe3O4/PLLA Nanofibers for Bone Tissue Engineering Applications. Polymers, 2018. 10(7).

176. Lee, S., et al., Lactic acid assisted fabrication of bioactive three-dimensional PLLA/ $\beta$-TCP fibrous scaffold for biomedical application. Chemical Engineering Journal, 2018. 347: p. 771-781.

177. Zhou, T., et al., Electrospun Poly(3-hydroxybutyrate-co-4hydroxybutyrate)/Graphene Oxide Scaffold: Enhanced Properties and Promoted in Vivo Bone Repair in Rats. ACS Applied Materials \& Interfaces, 2017. 9(49): p. 42589-42600.

178. Jaganathan, S.K. and M.P. Mani, Single-stage synthesis of electrospun polyurethane scaffold impregnated with zinc nitrate nanofibers for wound healing applications. 2019. 136(3): p. 46942.

179. M.P, D., et al., Hybrid electrospun fibers based on TPU-PDMS and spherical nanohydroxyapatite for bone tissue engineering. Materials Today Communications, 2018. 16: p. 264-273.

180. Enayati, M.S., et al., Development of electrospun poly (vinyl alcohol)-based bionanocomposite scaffolds for bone tissue engineering. Journal of Biomedical Materials Research Part A, 2018. 106(4): p. 1111-1120.

181. Ngadiman, H.N., et al., Novel Processing Technique to Produce Three Dimensional Polyvinyl Alcohol/Maghemite Nanofiber Scaffold Suitable for Hard Tissues. Polymers, 2018. 10(4).

182. Li, T.-T., et al., Mass-Production and Characterizations of Polyvinyl Alcohol/Sodium Alginate/Graphene Porous Nanofiber Membranes Using Needleless Dynamic Linear Electrospinning. Polymers, 2018. 10(10).

183. Kim, J.I., et al., The impact of humidity on the generation and morphology of the $3 D$ cotton-like nanofibrous piezoelectric scaffold via an electrospinning method. Materials Letters, 2019. 236: p. 510-513.

184. Saburi, E., et al., In vitro osteogenic differentiation potential of the human induced pluripotent stem cells augments when grown on Graphene oxide-modified nanofibers. Gene, 2019. 696: p. 72-79. 
185. Nalvuran, H., A.E. Elçin, and Y.M. Elçin, Nanofibrous silk fibroin/reduced graphene oxide scaffolds for tissue engineering and cell culture applications. International Journal of Biological Macromolecules, 2018. 114: p. 77-84.

186. Brito-Pereira, R., et al., Silk fibroin-magnetic hybrid composite electrospun fibers for tissue engineering applications. Composites Part B: Engineering, 2018. 141: p. 70-75.

187. Wang, S.-D., et al., Improving Antibacterial Activity and Biocompatibility of Bioinspired Electrospinning Silk Fibroin Nanofibers Modified by Graphene Oxide. ACS Omega, 2018. 3(1): p. 406-413.

188. Koppes, A., et al., Neurite outgrowth on electrospun PLLA fibers is enhanced by exogenous electrical stimulation. 2014. 11(4): p. 046002.

189. Fuh, Y.-K., et al., The control of cell orientation using biodegradable alginate fibers fabricated by near-field electrospinning. 2016. 62: p. 879-887.

190. Ozin, G.A., et al., Nanofabrication by self-assembly. Materials Today, 2009. 12(5): p. 12-23.

191. Wang, H.J., et al., One-step fabrication of tri-metallic PdCuAu nanothorn assemblies as an efficient catalyst for oxygen reduction reaction. Journal of Materials Chemistry A, 2018. 6(8): p. 3642-3648.

192. Chen, Y.H., et al., Light-enabled reversible self-assembly and tunable optical properties of stable hairy nanoparticles. Proceedings of the National Academy of Sciences of the United States of America, 2018. 115(7): p. E1391-E1400.

193. Long, H.W., et al., Self-Assembled Biomolecular ID Nanostructures for Aqueous Sodium-Ion Battery. Advanced Science, 2018. 5(3).

194. Yu, L., H.B. Wu, and X.W.D. Lou, Self-Templated Formation of Hollow Structures for Electrochemical Energy Applications. Accounts of Chemical Research, 2017. 50(2): p. 293-301.

195. Bengani, P., Y. Kou, and A. Asatekin, Zwitterionic copolymer self-assembly for fouling resistant, high flux membranes with size-based small molecule selectivity. Journal of Membrane Science, 2015. 493: p. 755-765.

196. Guven, S., et al., Multiscale assembly for tissue engineering and regenerative medicine. Trends in biotechnology, 2015. 33(5): p. 269-279.

197. Habibi, N., et al., Self-assembled peptide-based nanostructures: smart nanomaterials toward targeted drug delivery. Nano Today, 2016. 11(1): p. 41-60. 
198. Mann, S., Self-assembly and transformation of hybrid nano-objects and nanostructures under equilibrium and non-equilibrium conditions. Nature materials, 2009. 8(10): p. 781.

199. Ding, Y.P., et al., Electrospun Fibrous Architectures for Drug Delivery, Tissue Engineering and Cancer Therapy. Advanced Functional Materials, 2019. 29(2).

200. Aytac, Z., et al., Electrospinning of cyclodextrin/linalool-inclusion complex nanofibers: Fast-dissolving nanofibrous web with prolonged release and antibacterial activity. Food Chemistry, 2017. 231: p. 192-201.

201. Yao, Q.Q., et al., Three dimensional electrospun PCL/PLA blend nanofibrous scaffolds with significantly improved stem cells osteogenic differentiation and cranial bone formation. Biomaterials, 2017. 115: p. 115-127.

202. Liu, Q., et al., Recent advances in energy materials by electrospinning. Renewable \& Sustainable Energy Reviews, 2018. 81: p. 1825-1858.

203. Hong, C.H., et al., Electroactive bio-composite actuators based on cellulose acetate nanofibers with specially chopped polyaniline nanoparticles through electrospinning. Composites Science and Technology, 2013. 87: p. 135-141.

204. Zhai, Y., et al., Carbon dot/polyvinylpyrrolidone hybrid nanofibers with efficient solid-state photoluminescence constructed using an electrospinning technique. Nanotechnology, 2018. 29(2).

205. Shao, W.Y., et al., Polyster@MXene nanofibers-based yarn electrodes. Journal of Power Sources, 2018. 396: p. 683-690.

206. Liao, Y., et al., Progress in electrospun polymeric nanofibrous membranes for water treatment: Fabrication, modification and applications. Progress in Polymer Science, 2018. 77: p. 69-94.

207. Mehrpouya, F., et al., Nanostructured Electrospun Hybrid Graphene/Polyacrylonitrile Yarns. Nanomaterials, 2017. 7(10).

208. Chen, D., et al., Micro-Electromechanical Acoustic Resonator Coated with Polyethyleneimine Nanofibers for the Detection of Formaldehyde Vapor. Micromachines, 2018. 9(2).

209. Li, R., et al., Novel SA@Ca2+/RCSPs core-shell structure nanofibers by electrospinning for wound dressings. Rsc Advances, 2018. 8(28): p. 15558-15566.

210. Simonet, M., et al., Ultraporous 3D polymer meshes by low-temperature electrospinning: Use of ice crystals as a removable void template. Polymer Engineering and Science, 2007. 47(12): p. 2020-2026. 
211. Sun, B., et al., Self-assembly of a three-dimensional fibrous polymer sponge by electrospinning. Nanoscale, 2012. 4(6): p. 2134-2137.

212. Wang, H., et al., Direct-writing organic three-dimensional nanofibrous structure. Applied Physics a-Materials Science \& Processing, 2011. 102(2): p. 457-461.

213. Nedjari, S., G. Schlatter, and A. Hébraud, Thick electrospun honeycomb scaffolds with controlled pore size. Materials Letters, 2015. 142: p. 180-183.

214. Yan, G., et al., Self-Assembly of Electrospun Polymer Nanofibers: A General Phenomenon Generating Honeycomb-Patterned Nanofibrous Structures. Langmuir, 2011. 27(8): p. 4285-4289.

215. Wright, L.D., et al., Fabrication and mechanical characterization of $3 D$ electrospun scaffolds for tissue engineering. Biomedical Materials, 2010. 5(5): p. 055006.

216. Zong, C., Packing, covering and tiling in two-dimensional spaces. Expositiones Mathematicae, 2014. 32(4): p. 297-364.

217. Zhao, L., et al., Honeycomb porous $\mathrm{MnO} 2$ nanofibers assembled from radially grown nanosheets for aqueous supercapacitors with high working voltage and energy density. Nano Energy, 2014. 4: p. 39-48.

218. Liu, Y., et al., Cardiomyocyte coculture on layered fibrous scaffolds assembled from micropatterned electrospun mats. Materials Science and Engineering: C, 2017. 81: p. 500-510.

219. Chen, X., et al., Honeycomb-like polysulphone/polyurethane nanofiber filter for the removal of organic/inorganic species from air streams. Journal of Hazardous Materials, 2018. 347: p. 325-333.

220. Thandavamoorthy, S., N. Gopinath, and S.S. Ramkumar, Self-assembled honeycomb polyurethane nanofibers. Journal of Applied Polymer Science, 2006. 101(5): p. 3121-3124.

221. Wittmer, C.R., et al., Well-organized 3D nanofibrous composite constructs using cooperative, effects between electrospinning and electrospraying. Polymer, 2014. 55(22): p. 5781-5787.

222. Lavielle, N., et al., Structuring and Molding of Electrospun Nanofibers: Effect of Electrical and Topographical Local Properties of Micro-Patterned Collectors. Macromolecular Materials and Engineering, 2012. 297(10): p. 958-968.

223. Reis, T.C., I.J. Correia, and A. Aguiar-Ricardo, Electrodynamic tailoring of selfassembled three-dimensional electrospun constructs. Nanoscale, 2013. 5(16): p. 75287536. 
224. Nedjari, S., F. Awaja, and G. Altankov, Three Dimensional Honeycomb Patterned Fibrinogen Based Nanofibers Induce Substantial Osteogenic Response of Mesenchymal Stem Cells. Scientific Reports, 2017. 7(1): p. 15947.

225. Shacklette, L.W. and C.C. Han, Solubility and Dispersion Characteristics of Polyaniline. MRS Proceedings, 1993. 328: p. 157.

226. Bordes, C., et al., Determination of poly(e-caprolactone) solubility parameters: Application to solvent substitution in a microencapsulation process. International Journal of Pharmaceutics, 2010. 383(1): p. 236-243.

227. Stejskal, J., P. Kratochvíl, and N. Radhakrishnan, Polyaniline dispersions 2. UVVis absorption spectra. Vol. 61. 1993. 225-231.

228. Haider, A., S. Haider, and I.-K. Kang, A comprehensive review summarizing the effect of electrospinning parameters and potential applications of nanofibers in biomedical and biotechnology. Arabian Journal of Chemistry, 2015.

229. Yarin, A.L., S. Koombhongse, and D.H. Reneker, Taylor cone and jetting from liquid droplets in electrospinning of nanofibers. Journal of applied physics, 2001. 90(9): p. 4836-4846.

230. Persano, L., et al., Industrial upscaling of electrospinning and applications of polymer nanofibers: a review. Macromolecular Materials and Engineering, 2013. 298(5): p. 504-520.

231. Sun, B., et al., Self-assembly of a three-dimensional fibrous polymer sponge by electrospinning. Nanoscale, 2012. 4(6): p. 2134-2137.

232. STANGER, J., et al., EFFECT OF CHARGE DENSITY ON THE TAYLOR CONE IN ELECTROSPINNING. International Journal of Modern Physics B, 2009. 23(06n07): p. 1956-1961.

233. Achterberg, V.F., et al., The Nano-Scale Mechanical Properties of the Extracellular Matrix Regulate Dermal Fibroblast Function. Journal of Investigative Dermatology, 2014. 134(7): p. 1862-1872.

234. Mauck, R.L., et al., Engineering on the Straight and Narrow: The Mechanics of Nanofibrous Assemblies for Fiber-Reinforced Tissue Regeneration. Tissue Engineering. Part B, Reviews, 2009. 15(2): p. 171-193.

235. Pak, I., M. Kanthilal, and E. Darling, The Role of Cellular Mechanical Properties in Microenvironment-Dependent Behavior. Biophysical Journal, 2015. 108(2): p. 141a.

236. Darby, I.A., et al., Fibroblasts and myofibroblasts in wound healing. 2014. 7: p. 301. 
237. Place, A.E., S. Jin Huh, and K. Polyak, The microenvironment in breast cancer progression: biology and implications for treatment. Breast Cancer Research, 2011. 13(6): p. 227.

238. Cox, T.R. and J.T. Erler, Remodeling and homeostasis of the extracellular matrix: implications for fibrotic diseases and cancer. Disease models \& mechanisms, 2011. 4(2): p. $165-178$.

239. Thomas, C.H., et al., Engineering gene expression and protein synthesis by modulation of nuclear shape. Proceedings of the National Academy of Sciences of the United States of America, 2002. 99(4): p. 1972-1977.

240. Vergani, L., M. Grattarola, and C. Nicolini, Modifications of chromatin structure and gene expression following induced alterations of cellular shape. International Journal of Biochemistry \& Cell Biology, 2004. 36(8): p. 1447-1461.

241. Li, C., et al., Cell Type and Culture Condition-Dependent Alternative Splicing in Human Breast Cancer Cells Revealed by Splicing-Sensitive Microarrays. Cancer Research, 2006. 66(4): p. 1990-1999.

242. Petersen, O.W., et al., Interaction with basement membrane serves to rapidly distinguish growth and differentiation pattern of normal and malignant human breast epithelial cells. Proceedings of the National Academy of Sciences, 1992. 89(19): p. 90649068.

243. McCaffrey, L.M. and I.G. Macara, Epithelial organization, cell polarity and tumorigenesis. Trends in Cell Biology, 2011. 21(12): p. 727-735.

244. Semenza, G.L., The hypoxic tumor microenvironment: A driving force for breast cancer progression. Biochimica et Biophysica Acta (BBA)-Molecular Cell Research, 2016. 1863(3): p. 382-391.

245. Kim, H., et al., The hypoxic tumor microenvironment in vivo selects the cancer stem cell fate of breast cancer cells. Breast Cancer Research, 2018. 20(1): p. 16.

246. Roskelley, C., P. Desprez, and M. Bissell, Extracellular matrix-dependent tissuespecific gene expression in mammary epithelial cells requires both physical and biochemical signal transduction. Proceedings of the National Academy of Sciences, 1994. 91(26): p. 12378-12382.

247. Kenny, P.A., et al., The morphologies of breast cancer cell lines in threedimensional assays correlate with their profiles of gene expression. Molecular Oncology, 2007. 1(1): p. 84-96.

248. Rijal, G. and W. Li, 3D scaffolds in breast cancer research. Biomaterials, 2016. 81: p. 135-156. 
249. Kleinman, H.K. and G.R. Martin, Matrigel: Basement membrane matrix with biological activity. Seminars in Cancer Biology, 2005. 15(5): p. 378-386.

250. Carey, S.P., et al., Biophysical control of invasive tumor cell behavior by extracellular matrix microarchitecture. Biomaterials, 2012. 33(16): p. 4157-4165.

251. Feng, S., et al., Expansion of breast cancer stem cells with fibrous scaffolds. Integrative Biology, 2013. 5(5): p. 768-777.

252. Wolf, M.T., et al., A biologic scaffold-associated type 2 immune microenvironment inhibits tumor formation and synergizes with checkpoint immunotherapy. Science Translational Medicine, 2019. 11(477): p. eaat7973.

253. Dhandayuthapani, B., et al., Polymeric scaffolds in tissue engineering application: a review. International journal of polymer science, 2011. 2011.

254. Li, M., et al., Nanotopographical Surfaces for Regulating Cellular Mechanical Behaviors Investigated by Atomic Force Microscopy. ACS Biomaterials Science \& Engineering, 2019.

255. Tan, K.K.B., et al., Differential Cell Adhesion of Breast Cancer Stem Cells on Biomaterial Substrate with Nanotopographical Cues. Journal of Functional Biomaterials, 2015. 6(2): p. 241-258.

256. Kim, D.-H., et al., Biomechanical interplay between anisotropic re-organization of cells and the surrounding matrix underlies transition to invasive cancer spread. Scientific Reports, 2018. 8(1): p. 14210.

257. Park, J., D.-H. Kim, and A. Levchenko, Topotaxis: A New Mechanism of Directed Cell Migration in Topographic ECM Gradients. Biophysical Journal, 2018. 114(6): p. 1257-1263.

258. Sunyer, R., et al., Collective cell durotaxis emerges from long-range intercellular force transmission. Science, 2016. 353(6304): p. 1157.

259. Dvir, T., et al., Nanotechnological strategies for engineering complex tissues. Nature Nanotechnology, 2010. 6: p. 13.

260. Shieh, A.C., Biomechanical Forces Shape the Tumor Microenvironment. Annals of Biomedical Engineering, 2011. 39(5): p. 1379-1389.

261. Woodruff, M.A. and D.W. Hutmacher, The return of a forgotten polymerPolycaprolactone in the 21st century. Progress in Polymer Science, 2010. 35(10): p. 12171256. 
262. Ku, S.H., S.H. Lee, and C.B. Park, Synergic effects of nanofiber alignment and electroactivity on myoblast differentiation. Biomaterials, 2012. 33(26): p. 6098-6104.

263. Jose, M.V., et al., Aligned Bioactive Multi-Component Nanofibrous Nanocomposite Scaffolds for Bone Tissue Engineering. Macromolecular Bioscience, 2010. 10(4): p. 433444.

264. Conklin, M.W., et al., Aligned collagen is a prognostic signature for survival in human breast carcinoma. The American journal of pathology, 2011. 178(3): p. 1221-1232.

265. Broders-Bondon, F., et al., Mechanotransduction in tumor progression: The dark side of the force. The Journal of Cell Biology, 2018. 217(5): p. 1571.

266. Nagam Hanumantharao, S., et al., Electrospun acellular scaffolds for mimicking the natural anisotropy of the extracellular matrix. RSC Advances, 2019. 9(69): p. 4019040195.

267. Zhang, Q., et al., Bioinspired engineering of honeycomb structure - Using nature to inspire human innovation. Progress in Materials Science, 2015. 74: p. 332-400.

268. Calejo, M.T., et al., Honeycomb porous films as permeable scaffold materials for human embryonic stem cell-derived retinal pigment epithelium. Journal of Biomedical Materials Research Part A, 2016. 104(7): p. 1646-1656.

269. Huan, Z., et al., Characterization of a Honeycomb-Like Scaffold With Dielectrophoresis-Based Patterning for Tissue Engineering. IEEE Transactions on Biomedical Engineering, 2017. 64(4): p. 755-764.

270. Hanumantharao, S.N., C. Que, and S. Rao, Self-Assembly of 3D Nanostructures in Electrospun Polycaprolactone-Polyaniline Fibers and their Application as Scaffolds for Tissue Engineering. Materialia, 2019: p. 100296.

271. Heng, L., et al., Ordered Honeycomb Structural Interfaces for Anticancer Cells Growth. Langmuir, 2013. 29(48): p. 14947-14953.

272. Siegel, R.L., K.D. Miller, and A. Jemal, Cancer statistics, 2019. CA: a cancer journal for clinicians, 2019. 69(1): p. 7-34.

273. Schindelin, J., et al., Fiji: an open-source platform for biological-image analysis. Nature Methods, 2012. 9(7): p. 676-682.

274. Wang, X., et al., Crystalline morphology of electrospun poly ( $\varepsilon$ caprolactone)(PCL) nanofibers. Industrial \& Engineering Chemistry Research, 2013. 52(13): p. 4939-4949. 
275. Reneker, D.H., et al., Bending instability of electrically charged liquid jets of polymer solutions in electrospinning. Journal of Applied physics, 2000. 87(9): p. 45314547.

276. Nurfaizey, A.H., et al., Control of Spatial Deposition of Electrospun Fiber Using Electric Field Manipulation. Journal of Engineered Fibers and Fabrics, 2014. 9(1): p. 155164.

277. Putti, M., et al., Electrospinning poly(e-caprolactone) under controlled environmental conditions: Influence on fiber morphology and orientation. Polymer, 2015. 63: p. 189-195.

278. Bauer, A.L., T.L. Jackson, and Y. Jiang, A Cell-Based Model Exhibiting Branching and Anastomosis during Tumor-Induced Angiogenesis. Biophysical Journal, 2007. 92(9): p. 3105-3121.

279. Bauer, A.L., T.L. Jackson, and Y. Jiang, Topography of Extracellular Matrix Mediates Vascular Morphogenesis and Migration Speeds in Angiogenesis. PLOS Computational Biology, 2009. 5(7): p. e1000445.

280. Zussman, E., et al., Tensile deformation of electrospun nylon-6,6 nanofibers. Journal of Polymer Science Part B: Polymer Physics, 2006. 44(10): p. 1482-1489.

281. Arinstein, A., et al., Effect of supramolecular structure on polymer nanofibre elasticity. Nature Nanotechnology, 2007. 2: p. 59.

282. Lim, C.T., E.P.S. Tan, and S.Y. Ng, Effects of crystalline morphology on the tensile properties of electrospun polymer nanofibers. Applied Physics Letters, 2008. 92(14): p. 141908.

283. Chaudhuri, P.K., et al., Topography induces differential sensitivity on cancer cell proliferation via Rho-ROCK-Myosin contractility. Scientific Reports, 2016. 6: p. 19672.

284. Li, Q.S., et al., AFM indentation study of breast cancer cells. Biochemical and Biophysical Research Communications, 2008. 374(4): p. 609-613.

285. Cavo, M., et al., Microenvironment complexity and matrix stiffness regulate breast cancer cell activity in a 3D in vitro model. Scientific Reports, 2016. 6: p. 35367.

286. Dupont, S., et al., Role of YAP/TAZ in mechanotransduction. Nature, 2011. 474(7350): p. 179-183.

287. Mah, E.J., et al., Collagen density modulates triple-negative breast cancer cell metabolism through adhesion-mediated contractility. Scientific Reports, 2018. 8(1): p. 17094. 
288. Lin, F., et al., Substrate Stiffness Coupling TGF- $\beta 1$ Modulates Migration and Traction Force of MDA-MB-231 Human Breast Cancer Cells in Vitro. ACS Biomaterials Science \& Engineering, 2018. 4(4): p. 1337-1345.

289. Wei, S.C., et al., Matrix stiffness drives epithelial-mesenchymal transition and tumour metastasis through a TWIST1-G3BP2 mechanotransduction pathway. Nature Cell Biology, 2015. 17: p. 678.

290. Spill, F., et al., Impact of the physical microenvironment on tumor progression and metastasis. Current Opinion in Biotechnology, 2016. 40: p. 41-48.

291. Liotta, L.A., J. Kleinerman, and G.M. Saldel, The Significance of Hematogenous Tumor Cell Clumps in the Metastatic Process. Cancer Research, 1976. 36(3): p. 889.

292. Satoyoshi, R., et al., Asporin activates coordinated invasion of scirrhous gastric cancer and cancer-associated fibroblasts. Oncogene, 2015. 34(5): p. 650-660.

293. Rubashkin, M.G., et al., Force Engages Vinculin and Promotes Tumor Progression by Enhancing PI3K Activation of Phosphatidylinositol (3,4,5)-Triphosphate. Cancer Research, 2014. 74(17): p. 4597.

294. Jeon, H., et al., Directing cell migration and organization via nanocrater-patterned cell-repellent interfaces. Nature Materials, 2015. 14: p. 918.

295. Polacheck, W.J., I.K. Zervantonakis, and R.D. Kamm, Tumor cell migration in complex microenvironments. Cellular and Molecular Life Sciences, 2013. 70(8): p. 13351356.

296. Prendergast, P., R. Huiskes, and K. Søballe, Biophysical stimuli on cells during tissue differentiation at implant interfaces. Journal of biomechanics, 1997. 30(6): p. 539548.

297. Carey, S.P., et al., Mechanobiology of tumor invasion: engineering meets oncology. Critical reviews in oncology/hematology, 2012. 83(2): p. 170-183.

298. Oudin, M.J. and V.M. Weaver. Physical and chemical gradients in the tumor microenvironment regulate tumor cell invasion, migration, and metastasis. in Cold Spring Harbor symposia on quantitative biology. 2016. Cold Spring Harbor Laboratory Press.

299. Case, A., et al., Identification of prognostic collagen signatures and potential therapeutic stromal targets in canine mammary gland carcinoma. PLOS one, 2017. 12(7).

300. Plodinec, M., et al., The nanomechanical signature of breast cancer. Nature nanotechnology, 2012. 7(11): p. 757. 
301. Saito, T., et al., Aberrant collagen cross-linking in human oral squamous cell carcinoma. Journal of dental research, 2019. 98(5): p. 517-525.

302. Provenzano, P.P., et al., Collagen density promotes mammary tumor initiation and progression. BMC medicine, 2008. 6(1): p. 11.

303. Zhang, S.X., L. Liu, and W. Zhao, Targeting biophysical cues: a niche approach to study, diagnose, and treat cancer. Trends in cancer, 2018. 4(4): p. 268-271.

304. Kharkar, P.M., K.L. Kiick, and A.M. Kloxin, Designing degradable hydrogels for orthogonal control of cell microenvironments. Chemical Society Reviews, 2013. 42(17): p. 7335-7372.

305. Grover, C.N., et al., Crosslinking and composition influence the surface properties, mechanical stiffness and cell reactivity of collagen-based films. Acta Biomaterialia, 2012. 8(8): p. 3080-3090.

306. Slater, K., J. Partridge, and H. Nadivada, Tuning the Elastic Moduli of Corning® Matrigel ${ }^{\circledR}$ and Collagen I $3 D$ Matrices by Varying the Protein Concentration. Corning. Available online at: https://www. com/media/worldwide/cls/documents/applications/CLS-AC-AN-449 DL. pdf, 2017.

307. Xu, H., X. Liu, and W. Le, Recent advances in microfluidic models for cancer metastasis research. TrAC Trends in Analytical Chemistry, 2018. 105: p. 1-6.

308. Aisenbrey, E.A. and W.L. Murphy, Synthetic alternatives to Matrigel. Nature Reviews Materials, 2020.

309. Thuau, D., et al., Engineering polymer MEMS using combined microfluidic pervaporation and micro-molding. Microsystems \& nanoengineering, 2018. 4(1): p. 1-8.

310. Limongi, T., et al., Fabrication and applications of micro/nanostructured devices for tissue engineering. Nano-micro letters, 2017.9(1): p. 1.

311. Tseng, P., et al., Directed assembly of bio-inspired hierarchical materials with controlled nanofibrillar architectures. Nature nanotechnology, 2017. 12(5): p. 474-480.

312. Dvir, T., et al., Nanotechnological strategies for engineering complex tissues. Nature nanotechnology, 2011. 6(1): p. 13.

313. Yip, A.K., et al., Anisotropic traction stresses and focal adhesion polarization mediates topography-induced cell elongation. Biomaterials, 2018. 181: p. 103-112.

314. Hanumantharao, S.N., et al., Engineered Three-Dimensional Scaffolds Modulating Fate of Breast Cancer Cells Using Stiffness and Morphology Related Cell Adhesion. IEEE Open Journal of Engineering in Medicine and Biology, 2020. 1: p. 41-48. 
315. Zuiverloon, T.C.M., et al., Systematic Review: Characteristics and Preclinical Uses of Bladder Cancer Cell Lines. Bladder cancer (Amsterdam, Netherlands), 2018. 4(2): p. 169-183.

316. Sobczuk, P., et al., Choosing The Right Animal Model for Renal Cancer Research. Translational Oncology, 2020. 13(3): p. 100745.

317. Brodaczewska, K.K., et al., Choosing the right cell line for renal cell cancer research. Molecular Cancer, 2016. 15(1): p. 83.

318. Schindelin, J., et al., Fiji: an open-source platform for biological-image analysis. Nature methods, 2012. 9(7): p. 676-682.

319. Alfano, M., et al., Linearized texture of three-dimensional extracellular matrix is mandatory for bladder cancer cell invasion. Scientific Reports, 2016. 6(1): p. 36128.

320. Wong, S.-C., A. Baji, and S. Leng, Effect of fiber diameter on tensile properties of electrospun poly(e-caprolactone). Polymer, 2008. 49(21): p. 4713-4722.

321. Czekanska, E.M., Assessment of Cell Proliferation with Resazurin-Based Fluorescent Dye, in Mammalian Cell Viability: Methods and Protocols, M.J. Stoddart, Editor. 2011, Humana Press: Totowa, NJ. p. 27-32.

322. Pan, T., et al., Three-dimensional (3D) culture of bone-derived human 786-O renal cell carcinoma retains relevant clinical characteristics of bone metastases. Cancer Letters, 2015. 365(1): p. 89-95.

323. Mierke, C.T., et al., Integrin $\alpha 5 \beta 1$ facilitates cancer cell invasion through enhanced contractile forces. Journal of Cell Science, 2011. 124(3): p. 369-383.

324. Mierke, C.T., The integrin alphav beta 3 increases cellular stiffness and cytoskeletal remodeling dynamics to facilitate cancer cell invasion. New Journal of Physics, 2013. 15(1): p. 015003.

325. Strohmeyer, N., et al., Fibronectin-bound $\alpha 5 \beta 1$ integrins sense load and signal to reinforce adhesion in less than a second. Nature Materials, 2017. 16(12): p. 1262-1270.

326. Wisdom, K.M., et al., Matrix mechanical plasticity regulates cancer cell migration through confining microenvironments. Nature Communications, 2018. 9(1): p. 4144.

327. De Pascalis, C. and S. Etienne-Manneville, Single and collective cell migration: the mechanics of adhesions. Molecular biology of the cell, 2017. 28(14): p. 1833-1846.

328. Kristal-Muscal, R., et al., Mechanical Interaction of Metastatic Cancer Cells with a Soft Gel. Procedia IUTAM, 2015. 12: p. 211-219. 
329. Rianna, C. and M. Radmacher, Influence of microenvironment topography and stiffness on the mechanics and motility of normal and cancer renal cells. Nanoscale, 2017. 9(31): p. 11222-11230.

330. Chitcholtan, K., et al., Differences in growth properties of endometrial cancer in three dimensional (3D) culture and 2D cell monolayer. Experimental Cell Research, 2013. 319(1): p. 75-87.

331. Krakhmal, N.V., et al., Cancer Invasion: Patterns and Mechanisms. Acta naturae, 2015. 7(2): p. 17-28.

332. Wolf, K., et al., Physical limits of cell migration: control by ECM space and nuclear deformation and tuning by proteolysis and traction force. Journal of Cell Biology, 2013. 201(7): p. 1069-1084.

333. Park, J., et al., Switch-like enhancement of epithelial-mesenchymal transition by YAP through feedback regulation of WTI and Rho-family GTPases. Nature communications, 2019. 10(1): p. 2797-2797.

334. Glube, N., et al., Caki-1 cells represent an in vitro model system for studying the human proximal tubule epithelium. Nephron Exp Nephrol, 2007. 107(2): p. e47-56.

335. Breuksch, I., et al., Integrin a5 triggers the metastatic potential in renal cell carcinoma. Oncotarget, 2017. 8(64): p. 107530-107542.

336. Su, G., et al., The effect of forced growth of cells into $3 D$ spheres using low attachment surfaces on the acquisition of stemness properties. Biomaterials, 2013. 34(13): p. 3215-3222.

337. Tilghman, R.W., et al., Matrix rigidity regulates cancer cell growth and cellular phenotype. PloS one, 2010. 5(9): p. e12905-e12905.

338. Zhao, D., et al., Substrate stiffness regulated migration and angiogenesis potential of A549 cells and HUVECs. Journal of Cellular Physiology, 2018. 233(4): p. 3407-3417.

339. Tee, S.-Y., et al., Cell Shape and Substrate Rigidity Both Regulate Cell Stiffness. Biophysical Journal, 2011. 100(5): p. L25-L27.

340. Saito, T., et al., Correlation between integrin alpha 5 expression and the malignant phenotype of transitional cell carcinoma. British Journal of Cancer, 1996. 73(3): p. 327331.

341. Heyder, C., et al., Role of the $\beta 1$-integrin subunit in the adhesion, extravasation and migration of T24 human bladder carcinoma cells. Clinical \& Experimental Metastasis, 2005. 22(2): p. 99-106. 
342. Alfano, M., et al., Linearized texture of three-dimensional extracellular matrix is mandatory for bladder cancer cell invasion. Scientific reports, 2016. 6: p. 36128-36128.

343. Raczkowska, J. and S. Prauzner-Bechcicki, Discrimination between HCV29 and T24 by controlled proliferation of cells co-cultured on substrates with different elasticity. Journal of the Mechanical Behavior of Biomedical Materials, 2018. 88: p. 217-222.

344. Han, H., et al., Hippo signaling dysfunction induces cancer cell addiction to YAP. Oncogene, 2018. 37(50): p. 6414-6424.

345. Aragona, M., et al., A Mechanical Checkpoint Controls Multicellular Growth through YAP/TAZ Regulation by Actin-Processing Factors. Cell, 2013. 154(5): p. 10471059.

346. Khandelwal, M., et al., RASSF1A-Hippo pathway link in patients with urothelial carcinoma of bladder: plausible therapeutic target. Molecular and Cellular Biochemistry, 2020. 464(1): p. 51-63.

347. Pavlova, N.N. and C.B. Thompson, The Emerging Hallmarks of Cancer Metabolism. Cell metabolism, 2016. 23(1): p. 27-47.

348. Gouirand, V., F. Guillaumond, and S. Vasseur, Influence of the Tumor Microenvironment on Cancer Cells Metabolic Reprogramming. Frontiers in oncology, 2018. 8: p. 117-117.

349. Sreedhar, A. and Y. Zhao, Dysregulated metabolic enzymes and metabolic reprogramming in cancer cells (Review). Biomed Rep, 2018. 8(1): p. 3-10.

350. Fiaschi, T., et al., Reciprocal metabolic reprogramming through lactate shuttle coordinately influences tumor-stroma interplay. Cancer research, 2012. 72(19): p. 51305140 .

351. Beloribi-Djefaflia, S., S. Vasseur, and F. Guillaumond, Lipid metabolic reprogramming in cancer cells. Oncogenesis, 2016. 5(1): p. e189-e189.

352. Soga, T., Cancer metabolism: Key players in metabolic reprogramming. Cancer Science, 2013. 104(3): p. 275-281.

353. DeBerardinis, R.J., et al., The biology of cancer: metabolic reprogramming fuels cell growth and proliferation. Cell metabolism, 2008. 7(1): p. 11-20.

354. Vander Heiden, M.G., L.C. Cantley, and C.B. Thompson, Understanding the Warburg effect: the metabolic requirements of cell proliferation. science, 2009. 324(5930): p. 1029-1033. 
355. Kim, J.-w. and C.V. Dang, Cancer's molecular sweet tooth and the Warburg effect. Cancer research, 2006. 66(18): p. 8927-8930.

356. Zhang, T.-B., et al., Inhibition of glucose-transporter 1 (GLUT-1) expression reversed Warburg effect in gastric cancer cell MKN45. International journal of clinical and experimental medicine, 2015. 8(2): p. 2423.

357. Singer, K., et al., Warburg phenotype in renal cell carcinoma: High expression of glucose-transporter 1 (GLUT-1) correlates with low CD8+ T-cell infiltration in the tumor. International journal of cancer, 2011. 128(9): p. 2085-2095.

358. Macheda, M.L., S. Rogers, and J.D. Best, Molecular and cellular regulation of glucose transporter (GLUT) proteins in cancer. J Cell Physiol, 2005. 202(3): p. 654-62.

359. Krzeslak, A., et al., Expression of GLUT1 and GLUT3 glucose transporters in endometrial and breast cancers. Pathol Oncol Res, 2012. 18(3): p. 721-8.

360. Augustin, R., The protein family of glucose transport facilitators: It's not only about glucose after all. IUBMB Life, 2010. 62(5): p. 315-33.

361. Santinon, G., E. Enzo, and S. Dupont, The sweet side of YAP/TAZ. Cell cycle (Georgetown, Tex.), 2015. 14(16): p. 2543-2544.

362. Perestrelo, T., et al., Metabolic and Mechanical Cues Regulating Pluripotent Stem Cell Fate. Trends in Cell Biology, 2018. 28(12): p. 1014-1029.

363. Yang, S., et al., CDK1 Phosphorylation of YAP Promotes Mitotic Defects and Cell Motility and Is Essential for Neoplastic Transformation. Cancer Research, 2013. 73(22): p. 6722 .

364. Wang, W., et al., AMPK modulates Hippo pathway activity to regulate energy homeostasis. Nature Cell Biology, 2015. 17(4): p. 490-499.

365. Mo, J.-S., et al., Cellular energy stress induces AMPK-mediated regulation of YAP and the Hippo pathway. Nature Cell Biology, 2015. 17(4): p. 500-510.

366. Koo, J.H. and K.-L. Guan, Interplay between YAP/TAZ and Metabolism. Cell Metabolism, 2018. 28(2): p. 196-206.

367. Zwarts, I., et al., Identification of the fructose transporter GLUT5 (SLC2A5) as a novel target of nuclear receptor LXR. Scientific Reports, 2019. 9(1): p. 9299.

368. Weng, Y., et al., Fructose fuels lung adenocarcinoma through GLUT5. Cell Death \& Disease, 2018. 9(5): p. 557. 
369. Medina Villaamil, V., et al., Fructose transporter GLUT5 expression in clear renal cell carcinoma. Oncol Rep, 2011. 25(2): p. 315-23.

370. Ma, C., et al., Citrate-based materials fuel human stem cells by metabonegenic regulation. Proceedings of the National Academy of Sciences, 2018. 115(50): p. E11741.

371. Begoyan, V.V., et al., Multicolor GLUT5-permeable fluorescent probes for fructose transport analysis. Chemical Communications, 2018. 54(31): p. 3855-3858.

372. Ainsley, J., et al., Integrating molecular probes and molecular dynamics to reveal binding modes of GLUT5 activatory and inhibitory ligands. Chemical Communications, 2018. 54(71): p. 9917-9920.

373. Kannan, S., et al., Metabolism-driven high-throughput cancer identification with GLUT5-specific molecular probes. Biosensors, 2018. 8(2): p. 39.

374. Woelfle, U., et al., Down-regulated expression of cytokeratin 18 promotes progression of human breast cancer. Clinical cancer research, 2004. 10(8): p. 2670-2674.

375. Zamora-León, S.P., et al., Expression of the fructose transporter GLUT5 in human breast cancer. Proceedings of the National Academy of Sciences, 1996. 93(5): p. 18471852.

376. Godoy, A., et al., Differential subcellular distribution of glucose transporters GLUT1-6 and GLUT9 in human cancer: ultrastructural localization of GLUT1 and GLUT5 in breast tumor tissues. Journal of cellular physiology, 2006. 207(3): p. 614-627.

377. Park, C., et al., In situ Localization of Molecules in Crosslinked Particles during Electrohydrodynamic Process: Simple Route to Produce Microcapsules and Fibers with Controlled Release. Macromolecular Rapid Communications, 2010. 31(19): p. 1713-1718.

378. Metallo, Christian M. and Matthew G. Vander Heiden, Understanding Metabolic Regulation and Its Influence on Cell Physiology. Molecular Cell, 2013. 49(3): p. 388-398.

379. Vara, J.Á.F., et al., PI3K/Akt signalling pathway and cancer. Cancer treatment reviews, 2004. 30(2): p. 193-204.

380. Gatza, M.L., et al., A pathway-based classification of human breast cancer. Proceedings of the National Academy of Sciences, 2010. 107(15): p. 6994-6999.

381. Sabbatinelli, J., et al., Where Metabolism Meets Senescence: Focus on Endothelial Cells. Frontiers in Physiology, 2019. 10(1523).

382. Yao, G.-d., et al., Blocking the utilization of glucose induces the switch from senescence to apoptosis in pseudolaric acid B-treated human lung cancer cells in vitro. Acta Pharmacologica Sinica, 2017. 38(10): p. 1401-1411. 


\section{A Copyright and permissions}

Chapter 2: Published under the Creative commons License

Chapter 3: Copyright Information

Copyright

Clearance

Center

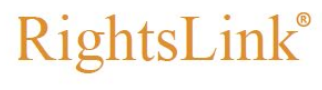

$\underset{\text { Home }}{\stackrel{?}{\text { Help }}}$

Email Support

Sign in

$\stackrel{2}{2}$

Create Account

$\begin{array}{ll} & \begin{array}{l}\text { Self-assembly of 3D nanostructures in electrospun } \\ \text { polycaprolactone-polyaniline fibers and their application as } \\ \text { Scaffolds for tissue engineering }\end{array} \\ \text { Author: Samerender Nagam Hanumantharao, Carolynn Que,Smitha Rao } \\ \text { Publication: Materialia } \\ \text { Publisher: Elsevier } \\ \text { Date: June 2019 } \\ \text { O 2019 Acta Materialia Inc. Published by Elsevier Ltd. All rights reserved. }\end{array}$

Please note that, as the author of this Elsevier article, you retain the right to include it in a thesis or dissertation, provided it is not published commercially. Permission is not required, but please ensure that you reference the journal as the original source. For more information on this and on your other retained rights, please visit: https://www.elsevier.com/about/our-business/policies/copyright\#Author-rights

BACK

CLOSE WINDOW

Chapter 4: Published under the Creative commons License 